\title{
Female versus male hearts
}

Citation for published version (APA):

Kimenai, D. (2019). Female versus male hearts: battle of the cardiac troponins. [Doctoral Thesis, Maastricht University]. Gildeprint Drukkerijen. https://doi.org/10.26481/dis.20190516dk

Document status and date:

Published: 01/01/2019

DOI:

$10.26481 /$ dis.20190516dk

Document Version:

Publisher's PDF, also known as Version of record

\section{Please check the document version of this publication:}

- A submitted manuscript is the version of the article upon submission and before peer-review. There can be important differences between the submitted version and the official published version of record.

People interested in the research are advised to contact the author for the final version of the publication, or visit the DOI to the publisher's website.

- The final author version and the galley proof are versions of the publication after peer review.

- The final published version features the final layout of the paper including the volume, issue and page numbers.

Link to publication

\footnotetext{
General rights rights.

- You may freely distribute the URL identifying the publication in the public portal. please follow below link for the End User Agreement:

www.umlib.nl/taverne-license

Take down policy

If you believe that this document breaches copyright please contact us at:

repository@maastrichtuniversity.nl

providing details and we will investigate your claim.
}

Copyright and moral rights for the publications made accessible in the public portal are retained by the authors and/or other copyright owners and it is a condition of accessing publications that users recognise and abide by the legal requirements associated with these

- Users may download and print one copy of any publication from the public portal for the purpose of private study or research.

- You may not further distribute the material or use it for any profit-making activity or commercial gain

If the publication is distributed under the terms of Article $25 \mathrm{fa}$ of the Dutch Copyright Act, indicated by the "Taverne" license above, 


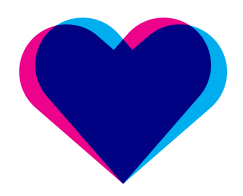

FEMALE VERSUS MALE HEARTS BATTLE OF THE CARDIAC TROPONINS

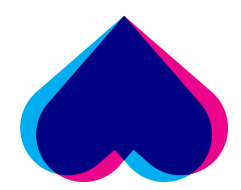




\section{FEMALE VERSUS MALE HEARTS - BATTLE OF THE CARDIAC TROPONINS}

Dorien M. Kimenai

Graphic design, cover and lay-out by Tomas Mahu

Printed by Gildeprint, Enschede, the Netherlands

ISBN [ 978946323593 8]

(C) Copyright Dorien M. Kimenai, Maastricht, 2019

No part of this book may be reproduced or transmitted in any form or by any means, without prior permission in writing by the author or, when appropriate, by the publishers of the publications. 


\section{FEMALE VERSUS MALE HEARTS BATTLE OF THE CARDIAC TROPONINS}

\section{PROEFSCHRIFT}

ter verkrijging van de graad van doctor aan de Universiteit Maastricht, op gezag van de Rector Magnificus, Prof. dr. Rianne M. Letschert volgens het besluit van het College van Decanen, in het openbaar te verdedigen op donderdag 16 mei 2019 om 16:0o uur

door

Dorothea Maria Kimenai 
PROMOTOR

Prof. dr. Otto Bekers

CO-PROMOTOR

Dr. Steven J.R. Meex

BEOORDELINGSCOMMISSIE

Prof. dr. Maurice P.A. Zeegers (voorzitter)

Prof. dr. Christa M. Cobbaert, LUMC, Leiden

Dr. Vanessa van Empel

Prof. dr. Manuela A. Joore

Prof. dr. Ron Kusters, Jeroen Bosch Ziekenhuis, 's Hertogenbosch

The research described in this thesis was supported by a grant of Stichting De Weijerhorst.

Financial support by the Dutch Heart Foundation for the publication of this thesis is gratefully acknowledged. 


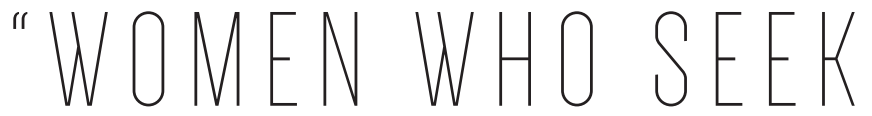

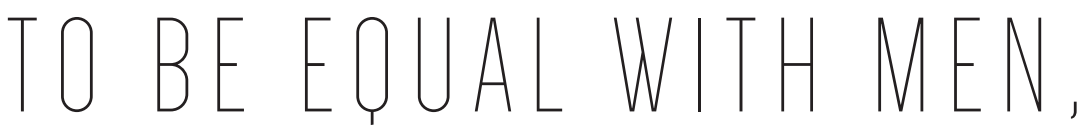

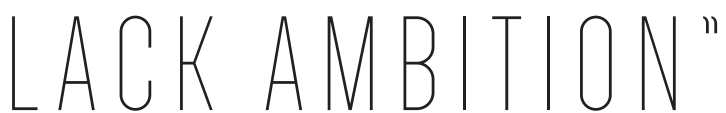

- Marilyn Monroe - 

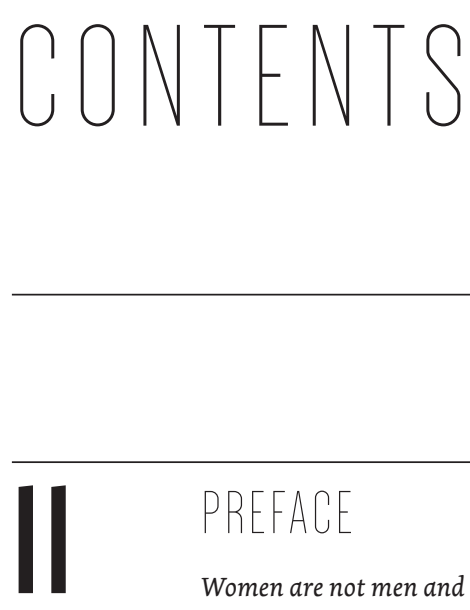

\section{PREFACE}

Women are not men and

men are not women,

fortunately

\section{6}

\section{CHAPTER 2}

Direct comparison of clinical decision limits for cardiac troponin I and $\mathrm{T}$

\section{CHAPTER 4 \\ Sex-Related Aspects of \\ Biomarkers in \\ Cardiac Disease \\ 78}

\section{CHAPTER I}

General introduction
46 CHAPTER 3

Sex-Specific Versus Overall Clinical Decision Limits for Cardiac Troponin I and T for the Diagnosis of Acute Myocardial Infarction: A Systematic Review 


\section{8}

Estimated Glomerular Filtration Rate and Albuminuria Are

Associated with Biomarkers

of Cardiac Injury in a

Population-Based Cohort Study:

The Maastricht Study

\section{5}

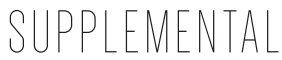

Summary

\section{0 CHAPTER 7 \\ General discussion}

188

SUPPLEMENTAL

Nederlandse samenvatting (Dutch summary) 


\section{PREFACE}

Women are not men and men are not women, fortunately. For decades there was no particular interest for sex differences in science. Today, animal studies are still mainly based on male-models. In respect of drug research, an important argument for male-only research in the past, was safety, e.g. risk of harm on (future) pregnancies. As a result, females were mostly excluded and underrepresented in prospective clinical trials. The latter has been changed, and according to current European law, clinical trials must include female individuals proportionate with the disease prevalence in the population, and also sex-specific effectiveness-and safety analyses are required.

Today, the urgent need to take potential sex differences into account has been more broadly recognized, e.g. by initiatives from WOMEN Inc., Dutch Society for Gender and Health, and awareness initiative Dress Red Day. However, in my profession as clinical epidemiologist, I feel that the main responsibility for this topic lies with all researchers conducting scientific research. At the start of a study each researcher should answer the (simple) question:

Are sex differences possibly relevant for this research question?

If the answer on this question is "no", than a sex-based study design is not a necessity. However, I still do recommend, if possible, to report sex-stratified analyses, e.g. in the supplemental files. Although the sex-specific analyses might not be sufficiently powered to make firm conclusions, future researchers will be able to conduct a systematic review including sex-specific analyses.

Last but not least, I embrace the diversity of the sexes by not ignoring their clinical relevant differences. Get inspired.

Dorien Kimenai 


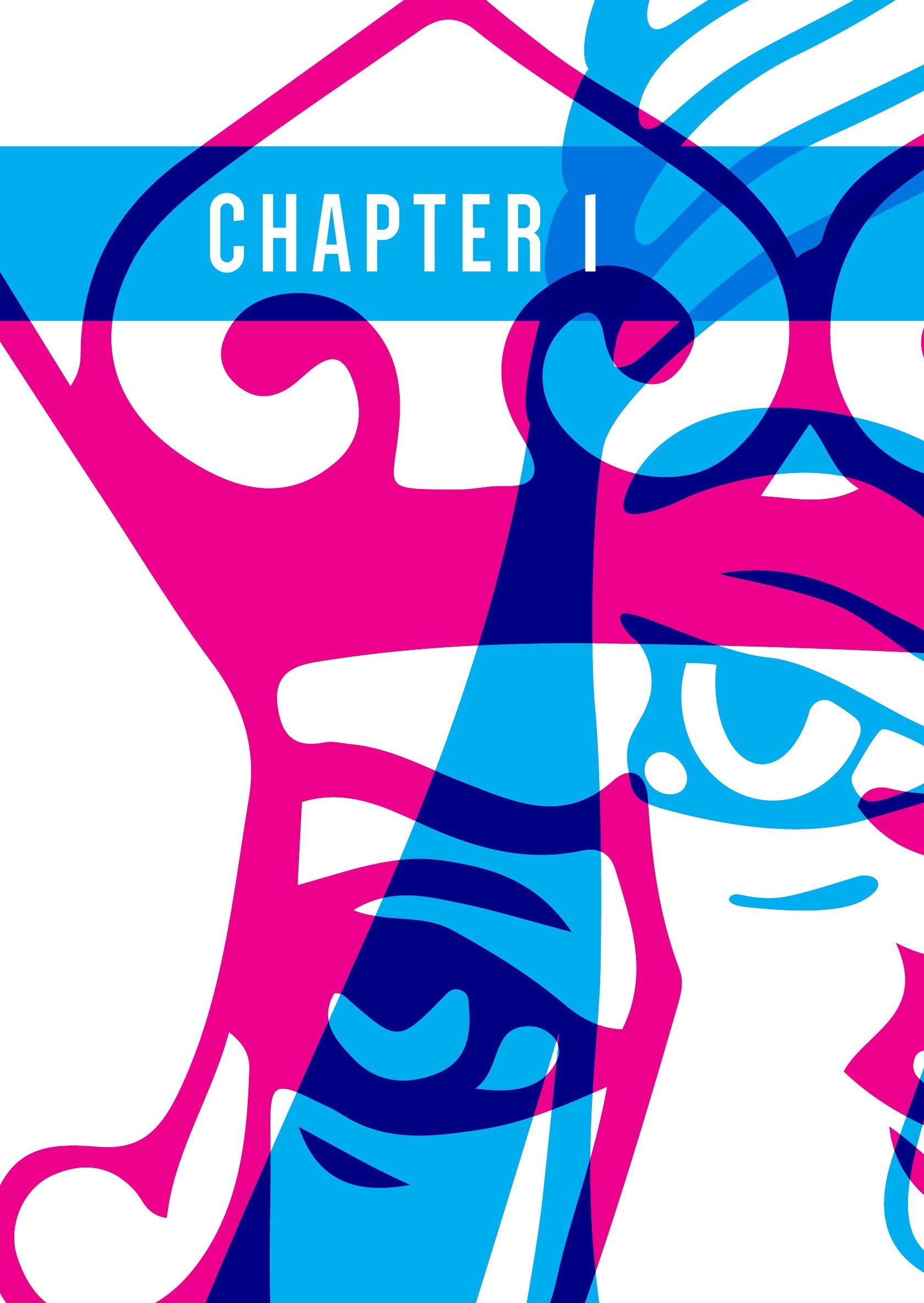





\section{CHAPTER I}

GENERAL INTRODUCTION 


\section{NOW LET'S TALK ABOUT SEX...AND CARDIAC TROPONINS}

\section{, EPIDEMIOLOGY OF ACUTE MYOCARDIAL INFARCTION STRATIFIED BY SEX}

An acute myocardial infarction (AMI), or in the more general term "heart attack", is defined by myocardial cell death owing to prolonged myocardial ischemia as a result of an acute imbalance between oxygen supply and demand. ${ }^{1}$ In the past, the general line of thought was that an AMI was more an issue for men than women, and AMI was therefore called a "men's disease". This was mainly based on the fact that the proportion of AMIs was higher for men than women.

The perception that AMI was a typical men's disease changed when the decline in cardiovascular disease (CVD) related deaths over the last three decades was less pronounced in women as compared with men. ${ }^{2}$ This trend was on the basis of growing interest in the female heart. To date, long-term survival rate after an AMI is still worse for women than men. A proportion of $23 \%$ versus $18 \%$ of women and men, respectively, die within one year after AMI, and this substantial survival gap is not well understood. ${ }^{3}$

In contrast to long-term survival rate after AMI, the number of hospital admissions for AMI is higher for men than women (23.123 versus 11.307) in the Netherlands. ${ }^{4}$ A higher proportion of men than women dies owing to AMI. In 2017, the absolute numbers (\%) of AMI related deaths, stratified by sex, were $2.858(16 \%)$ men and 2.215 $(11 \%)$ women (Table 1.1). ${ }^{4}$

It is important to note that it is not quite clear whether the observed difference in proportion of AMI related deaths between women and men is correct, or (partly) marked by the fact that misdiagnosis is hard to measure. A population-based cohort study reported a higher number of clinically unrecognized AMIs of $54 \%$ for women versus $33 \%$ for men, ${ }^{5}$ raising the awareness that AMI is more frequently missed in women than men. On the other hand, the biological (and social) differences between women and men may result in divergent risk profiles, and can explain the differences in AMI incidence between sexes. E.g. the sex hormone estrogen seems to have a protective role in the development of CVD in females, which might explain the fact that women with AMI are on average ten years older, which results in a lower prevalence of AMI diagnosis in women. ${ }^{6-9}$ 


\begin{tabular}{|c|c|c|c|c|c|}
\hline \multirow{2}{*}{$\begin{array}{r}\text { Table } 1.1 \\
\text { Numbers of AMI } \\
\text { related deaths in } \\
\text { the Netherlands, } \\
\text { stratified by sex } \\
\text { and age. }\end{array}$} & \multirow[b]{2}{*}{ Age (years) } & \multicolumn{2}{|c|}{ Women } & \multicolumn{2}{|c|}{ Men } \\
\hline & & $\begin{array}{l}\text { Number of } \\
\text { deaths (N) }\end{array}$ & $\begin{array}{l}\text { Mortality rate per } \\
\text { I00.000 people }\end{array}$ & $\begin{array}{l}\text { Number of } \\
\text { deaths (N) }\end{array}$ & $\begin{array}{l}\text { Mortality rate per } \\
\text { I00.000 people }\end{array}$ \\
\hline & $0-44$ & 13 & $<1$ & 49 & | \\
\hline & $45-54$ & 47 & 4 & $|6|$ & 12 \\
\hline & $55-64$ & 133 & 12 & 383 & 34 \\
\hline & $65-74$ & 271 & 30 & 715 & 81 \\
\hline & $75-84$ & 640 & $\| 9$ & 849 & 199 \\
\hline & $85-94$ & 956 & 426 & 630 & 571 \\
\hline & $95+$ & 155 & 835 & 71 & 1.526 \\
\hline & Total & 2.215 & 26 & 2.858 & 34 \\
\hline
\end{tabular}

\section{> DIAGNOSIS OF AMI}

The clinical presentation, electrocardiographic (ECG) findings and the presence of cardiac troponins ( $\mathrm{I}$ or $\mathrm{T}$ ) elevations are the cornerstones for the diagnosis of AMI (Figure 1.1).1,10 In case of ST-elevated myocardial infarction (STEMI), the diagnosis is based on ECG observations. For non-ST-elevated myocardial infarction (NSTEMI), the cardiac troponin algorithms and clinical presentation are the decisive elements for establishment of AMI diagnosis, which will be explained in more detail in the next paragraph.

The current European Society of Cardiology (ESC) guidelines do not recommend a sexspecific diagnostic algorithm for AMI. ${ }^{10}$ However, over the last years, sex differences became evident. ${ }^{11}$ E.g. the "typical" characteristics for AMI as having chest pain radiating

\footnotetext{
Figure 1.1
Diagnostic algorithm of AMI

Abbreviations:

ECG, electro-

cardiography; NSTEMI,

non-ST-elevated

myocardial

infarction;

STEMI,

ST-elevated

myocardial

infarction; $U A$,

unstable angina.
}
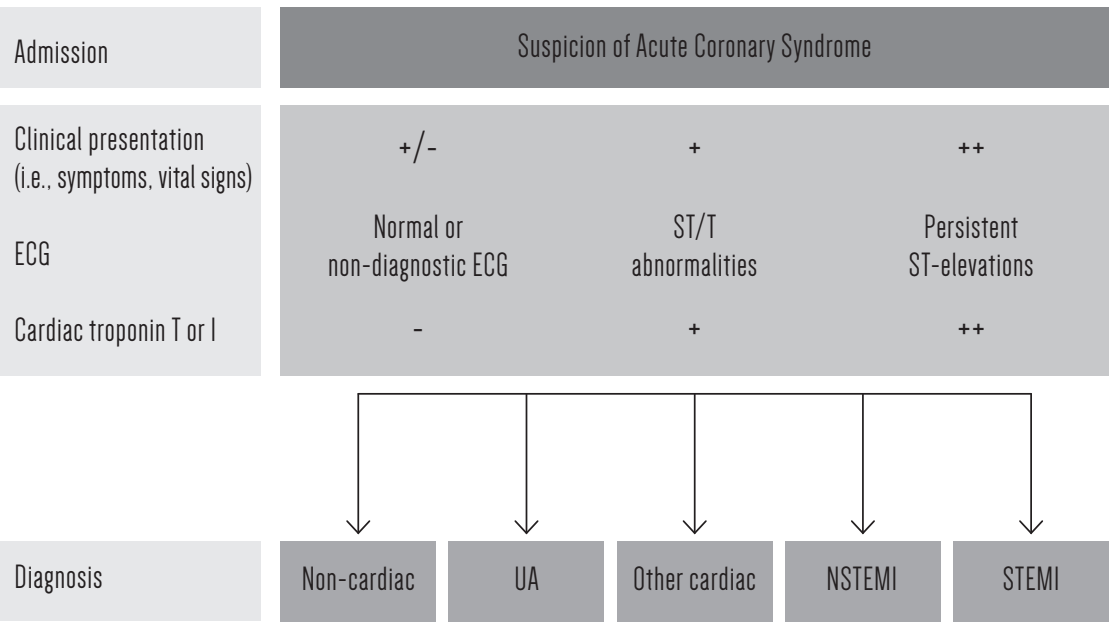
to the left arm and a conclusiveECG are not that typical for women, as they are less present in women than men. ${ }^{12-15}$ This suggests that the "typical" clinical presentation may not be sufficiently sensitive to recognize AMI in women, and hamper their diagnosis.

\section{> CARDIAC TROPONIN ALGORITHMS FOR DIAGNOSIS OF AMI}

Before going into detail on sex-related aspects of cardiac troponins, we will first explain the rationale behind the current diagnostic cardiac troponin algorithms for diagnosis of AMI. As stated above, the gold cardiac biomarker standard to establish AMI diagnosis in clinical practice are the cardiac troponins..$^{10,16}$ Cardiac troponin I and $\mathrm{T}$ are released in the bloodstream after cardiac injury, and owing to their high cardiac specificity, both cardiac troponins can be applied in clinical practice. ${ }^{10}$ In other words, hospitals use either a cardiac troponin I assay or cardiac troponin $\mathrm{T}$ assay, depending on the clinical analyzer series which is used in their laboratory.

Sensitivity of cardiac troponin assays has been improved over the last years, and it became feasible to measure troponin concentrations at a lower concentration range, even in presumably "healthy" individuals. The current guidelines recommend these so-called high sensitivity cardiac troponin (hs-cTn) assays over the contemporary troponin assays for diagnosis of AMI. ${ }^{10} \mathrm{~A}$ hs-cTn assay should meet two criteria $\left.{ }^{1}: 1\right)$ The $99^{\text {th }}$ percentile upper reference limit (URL) from a healthy reference population should be lower than the $10 \%$ coefficient of variation (10\% CV).2) At least $50 \%$ of healthy female individuals and $50 \%$ of healthy male individuals should have detectable troponin concentrations.

At this point, the $99^{\text {th }}$ percentile URL of hs-cTn derived from a healthy reference population is the consensus diagnostic threshold for AMI diagnosis. The present ESC guidelines recommend a oh/ $3 \mathrm{~h}$ algorithm which requires a significant rise or fall of hs-cTn with at least one value above the $99^{\text {th }}$ percentile URL from a healthy reference population..$^{10}$ In addition, a oh/ $1 \mathrm{~h}$ algorithm is included in the 2015 ESC guidelines which enables a faster rule-in and rule-out strategy. ${ }^{10}$ However, the oh/ $1 \mathrm{~h}$ algorithm requires other diagnostic hs-cTn assay-specific thresholds than $99^{\text {th }}$ percentile URLs of hs-cTn.

\section{, THE 99TH PERCENTILE URLS OF HS-CTN FOR DIAGNOSIS OF AMI}

As this thesis will mainly focus on the $99^{\text {th }}$ percentile URLs derived from a healthy reference population, one may get the impression that the $99^{\text {th }}$ percentile URL is a natural law for defining a diagnostic threshold in biomarker approaches, which it is actually not.

A diagnostic threshold is used to discriminate between healthy and diseased individuals. However, there are several methods to estimate the optimal diagnostic threshold of a biomarker for diagnosis an disease. In clinical 
chemistry, reference intervals are a commonly used method to discriminate between healthy and diseased individuals. ${ }^{17}$ In most cases a reference interval of a biomarker is calculated which comprises $95 \%$ of the values from a healthy reference population, and wherefrom accordingly, lower- and upper reference limits are determined. ${ }^{17}$ The reference interval can be determined based on a nonparametric approach or parametric approach. As the $99^{\text {th }}$ percentile URL of hs$\mathrm{cTn}$ is based on a non-parametric approach, the parametric approach is beyond the scope of this chapter. To encompass $95 \%$ of values of the total population, an interpercentile interval between $2.5^{\text {th }}$ and $97.5^{\text {th }}$ percentiles is used. This means that a percentage of $2.5 \%$ of values on each side of distribution tail falls outside the determined reference range. Hence, the $2.5^{\text {th }}$ and $75^{\text {th }}$ percentiles are defined as lower- and upper reference limits, respectively.

Accordingly, the diagnostic performance of a biomarker can be investigated by a Receiver Operating Characteristic (ROC) curve. Biomarkers can be compared with a "gold standard" reference test. The accuracy, defined by sensitivity and specificity, could be determined by the proportion of false positives (FP) and false negatives (FN). Accordingly, positive predicted value (PPV), an important measure for "rule in", and negative predictive value (NPV), and important safety measure for "rule out", can be calculated (Table 1.2). The ideal diagnostic threshold does not lead to an excessive amount of FP and FN. Based on the position of the biomarker in the diagnostic pathway, the optimal diagnostic threshold could be estimated by the preferred sensitivity/specificity ratio in relation to the clinical setting (prevalence of disease). For hs-cTn assays the rule of thumb is that they should require at least a PPV of $80 \%$, and a sensitivity $\geq 99 \%$ to avoid misdiagnosis. ${ }^{18,19}$

According to expert opinion, a consensus was reached in the 2011 ESC guidelines that diagnostic cutoffs of cardiac troponins should not be lower than $10 \% \mathrm{CV}$ concentrations to avoid misclassification. ${ }^{1}$ For contemporary cardiac troponin

\begin{tabular}{|c|c|c|c|c|}
\hline \multirow{2}{*}{$\begin{array}{r}\text { Table } 1.2 \\
\text { Accuracy of } \\
\text { a diagnostic } \\
\text { biomarker } \\
\text { approach. }\end{array}$} & & \multicolumn{3}{|c|}{ "Gold standard" reference test } \\
\hline & Biomarker approach & $\begin{array}{l}\text { Patient } \\
\text { Normal }\end{array}$ & $\begin{array}{l}\text { Patient } \\
\text { True Positive } \\
\text { False Negative }\end{array}$ & $\begin{array}{l}\text { Normal } \\
\text { False Positive } \\
\text { True Negative }\end{array}$ \\
\hline & Sensitivity & \multicolumn{3}{|c|}{ True Positive / True Positive + False Negative } \\
\hline & Specificity & \multicolumn{3}{|c|}{ True Negative/ True Negative + False Positive } \\
\hline & Positive Predicted Value & \multicolumn{3}{|c|}{ True Positive /(True Positive + False Positive) } \\
\hline & Negative Predicted Value & \multicolumn{3}{|c|}{ True Negative (True Negative + False Negative) } \\
\hline
\end{tabular}


assays the percentile method was mostly not feasible as they were restricted by their analytical properties. In that case, $10 \% \mathrm{CV}$ values of these cardiac troponin assays were chosen as diagnostic cutoff for AMI diagnosis. Most likely, during the implementation of hs-cTn assays, the precision did not reach the $10 \% \mathrm{CV}$ threshold requirement using $97.5^{\text {th }}$ percentile URLs, and therefore the $99^{\text {th }}$ percentile URLs of hs-cTn were chosen as alternative. Hence, now we understand the background of $99^{\text {th }}$ URLs for hs-cTn assays as diagnostic threshold for AMI, it is time to explain the two clinical uncertainties which are raised by using hscTnI and hs-cTnT in clinical practice. The main focus in this thesis will be on the hs-cTnI assay of Abbott and hs-cTnT of Roche, as they are defined as "highsensitivity" according to the above stated criteria. ${ }^{20}$

The first issue is that package-insert defined $99^{\text {th }}$ URLs of $26 \mathrm{ng} / \mathrm{L}$ for hs-cTnI and $14 \mathrm{ng} / \mathrm{L}$ for hs-cTnT are overall thresholds, not stratified by sex. The hscTn assays revealed previously unrecognized lower cardiac troponin levels in women than men. ${ }^{11}$ Thus far, it has not been systematically investigated whether the sex difference in cardiac troponin concentrations are of such relevance that this results into different female-specific $99^{\text {th }}$ percentile URLs and malespecific $99^{\text {th }}$ percentile URLs of hs-cTn. Secondly, $99^{\text {th }}$ URLs of hs-cTnI and hscTnT are derived from different "healthy" reference populations. ${ }^{21,22}$ A number of methodological aspects differ between clinically applied $99^{\text {th }}$ URLs of hs-cTnI and hs-cTnT, e.g. the definition of "healthy" reference population, constitution of reference population, sample size, statistical approach and handling of outliers. The impact of different methodological aspects on $99^{\text {th }}$ URL of hs-cTnI and hscTnT is thus far not systematically investigated.

At this point, hs-cTn is only incorporated in the algorithm for diagnosis of AMI, but hs-cTn might be useful for diagnosis and/or prognosis of other (cardiac) diseases. It is shown that even slightly elevated troponin levels predicts worse prognosis on CVD related morbidity and mortality. ${ }^{6}$ However, differences in subgroups in relation to cardiac troponins, but also a discordance between cardiac troponin I and $\mathrm{T}$ became apparent. ${ }^{23-25}$ Whether an elevated cardiac troponin concentration only reflects (subclinical) cardiac injury or also other non-cardiac pathology such as reduced kidney function is an ongoing discussion over the last years. Before hs-cTn assays were introduced, differences between troponin $\mathrm{T}$ and I levels were already demonstrated in chronic kidney disease patients, suggesting that reduced clearance may play a role in the divergence between troponin I and T. ${ }^{26} 27$ Hence, the additional load on troponin data that became available from hs-cTn assays could provide us better insight into 1) observed sex differences of the cardiac troponin levels, and to which extend this influences the $99^{\text {th }}$ URLs of hs-cTnI and hs-cTnI, and 2) discordance between hs-cTnI and hs-cTnT and their underlying mechanisms of cardiac troponin elevations. 


\section{$>$ OUTLINE OF THIS THESIS}

In this thesis we unravel two aspects:

\section{> FEMALE VERSUS MALE HEARTS}

Sex-related aspects of cardiac biomarkers, in particular the $99^{\text {th }}$ percentile upper reference limits of high-sensitivity cardiac troponins for the diagnosis of an acute myocardial infarction.

\section{> BATTLE OF THE CARDIAC TROPONINS}

The (dis)concordance between high-sensitivity cardiac troponin I and $\mathrm{T}$ in respect of $99^{\text {th }}$ percentile upper reference limits, and their underlying associations with cardiac injury and kidney function in the non-acute setting.

In chapter two we determined sex-specific and overall $99^{\text {th }}$ percentile URLs of hscTnI and hs-cTnT from a single healthy reference population derived from The Maastricht Study cohort. In chapter three we conducted a systematic review of the literature to investigate sex-specific versus overall clinical decision limits of hscTnI and hs-cTnT. In addition to sex differences, we evaluated whether clinically applied decision limits of hs-cTnI and hs-cTnT were biologically equivalent by investigating the $99^{\text {th }}$ percentile values of both cardiac troponins derived from single reference cohorts. Chapter four is an overview of the literature of sex differences in relation to cardiac biomarkers which are used for diagnosis and prognosis in acute cardiac care. The concordance between hs-cTnI and hscTnT in relation to cardiac injury, defined by ECG changes indicative for cardiac abnormalities, is investigated in The Maastricht Study cohort and described in chapter five. In chapter six we assessed and compared associations between kidney- and cardiac biomarkers. In chapter seven, we discussed the results of this thesis in the perspective of current knowledge. 
REFERENCES

1. Thygesen K, Alpert JS, Jaffe AS, et al. Fourth universal definition of myocardial infarction. European heart journal 2019;40(3):237-269.

2. Pagidipati NJ, Peterson ED. Acute coronary syndromes in women and men. Nat Rev Cardiol 2016;13(8):471-80.

3. Benjamin EJ, Blaha MJ, Chiuve SE, et al. Heart Disease and Stroke Statistics-2017 Update: A Report From the American Heart Association. Circulation 2017;135(10):e146-e603.

4. Buddeke J, Van Dis I, Visseren FLJ, Vaartjes I, Bots ML. Ziekte en stertfte aan hart- en vaatziekten. In: Buddeke J, Van Dis I, Visseren FLJ, Vaartjes I, Bots ML. Hart- en vaatziekten in Nederland 2017, cijfers over leefstijl, risicofactoren, ziekte en sterfte. Den Haag: Hartstichting, 2017.

5. de Torbal A, Boersma E, Kors JA, et al. Incidence of recognized and unrecognized myocardial infarction in men and women aged 55 and older: the Rotterdam Study. European heart journal 2006;27(6):729-36.

6. Twerenbold R, Boeddinghaus J, Nestelberger T, et al. Clinical Use of High-Sensitivity Cardiac Troponin in Patients With Suspected Myocardial Infarction. Journal of the American College of Cardiology 2017;70(8):996-1012.

7. Westerman S, Wenger NK. Women and heart disease, the underrecognized burden: sex differences, biases, and unmet clinical and research challenges. Clin Sci (Lond) 2016;130(8):551-63.

8. Donaldson C, Eder S, Baker C, et al. Estrogen attenuates left ventricular and cardiomyocyte hypertrophy by an estrogen receptor-dependent pathway that increases calcineurin degradation. Circ Res 2009;104(2):265-75.

9. Piro M, Della Bona R, Abbate A, et al. Sex-related differences in myocardial remodeling. Journal of the American College of Cardiology 2010;55(11):1057-65.

10. Roffi M, Patrono C, Collet JP, et al. 2015 ESC Guidelines for the management of acute coronary syndromes in patients presenting without persistent ST-segment elevation: Task Force for the Management of Acute Coronary Syndromes in Patients Presenting without Persistent ST-Segment Elevation of the European Society of Cardiology (ESC). European heart journal 2016;37(3):267-315.

11. Mingels A, Jacobs L, Michielsen E, et al. Reference population and marathon runner sera assessed by highly sensitive cardiac troponin $\mathrm{T}$ and commercial cardiac troponin $\mathrm{T}$ and $\mathrm{I}$ assays. Clinical chemistry 2009;55(1):101-8.

12. Canto JG, Goldberg RJ, Hand MM, et al. Symptom presentation of women with acute coronary syndromes: myth vs reality. Arch Intern Med 2007;167(22):2405-13. 
13. Elsaesser A, Hamm CW. Acute coronary syndrome: the risk of being female. Circulation 2004;109(5):565-7.

14. Madonis SM, Skelding KA, Roberts M. Management of acute coronary syndromes: special considerations in women. Heart 2017;103(20):1638-46.

15. Pilote L, Karp I. GENESIS-PRAXY (GENdEr and Sex determInantS of cardiovascular disease: From bench to beyond-Premature Acute Coronary SYndrome). Am Heart J 2012;163(5):741-46 e2.

16. Alpert JS, Thygesen K, Antman E, et al. Myocardial infarction redefined--a consensus document of The Joint European Society of Cardiology/American College of Cardiology Committee for the redefinition of myocardial infarction. Journal of the American College of Cardiology 2000;36(3):959-69.

17. Burtis CA, Bruns DA. Tietz Fundamentals of Clinical Chemistry and Molecular Diagnostics. Elsevier Health Sciences 2014.

18. Eggers KM, Jernberg T, Ljung L, et al. High-Sensitivity Cardiac Troponin-Based Strategies for the Assessment of Chest Pain Patients-A Review of Validation and Clinical Implementation Studies. Clinical chemistry 2018.

19. Than $M$, Herbert $M$, Flaws $D$, et al. What is an acceptable risk of major adverse cardiac event in chest pain patients soon after discharge from the Emergency Department?: a clinical survey. Int J Cardiol 2013;166(3):752-4.

20. Apple FS, Collinson PO, Biomarkers ITFoCAoC. Analytical characteristics of highsensitivity cardiac troponin assays. Clinical chemistry 2012;58(1):54-61.

21. Rogers J, Du S, Yen J, et al. Determination of a 99th percentile for the ARCHITECT STAT High Sensitive Troponin-I Immunoassay using a Robust Statistical Method. Clinical chemistry 2013;59(10):A225.

22. Saenger AK, Beyrau R, Braun S, et al. Multicenter analytical evaluation of a highsensitivity troponin $\mathrm{T}$ assay. Clinica chimica acta; international journal of clinical chemistry 2011;412(9-10):748-54.

23. Omland T, Pfeffer MA, Solomon SD, et al. Prognostic value of cardiac troponin I measured with a highly sensitive assay in patients with stable coronary artery disease. Journal of the American College of Cardiology 2013;61(12):1240-9.

24. deFilippi C, Seliger SL, Kelley W, et al. Interpreting cardiac troponin results from high-sensitivity assays in chronic kidney disease without acute coronary syndrome. Clinical chemistry 2012;58(9):1342-51.

25. Hijazi Z, Siegbahn A, Andersson U, et al. Comparison of cardiac troponins I and T measured with high-sensitivity methods for evaluation of prognosis in atrial fibrillation: an ARISTOTLE substudy. Clinical chemistry 2015;61(2):368-78.

26. Freda BJ, Tang WH, Van Lente F, et al. Cardiac troponins in renal insufficiency: review and clinical implications. Journal of the American College of Cardiology 2002;40(12):2065-71.

27. Abbas NA, John RI, Webb MC, et al. Cardiac troponins and renal function in nondialysis patients with chronic kidney disease. Clinical chemistry 2005;51(11):2059-66. 


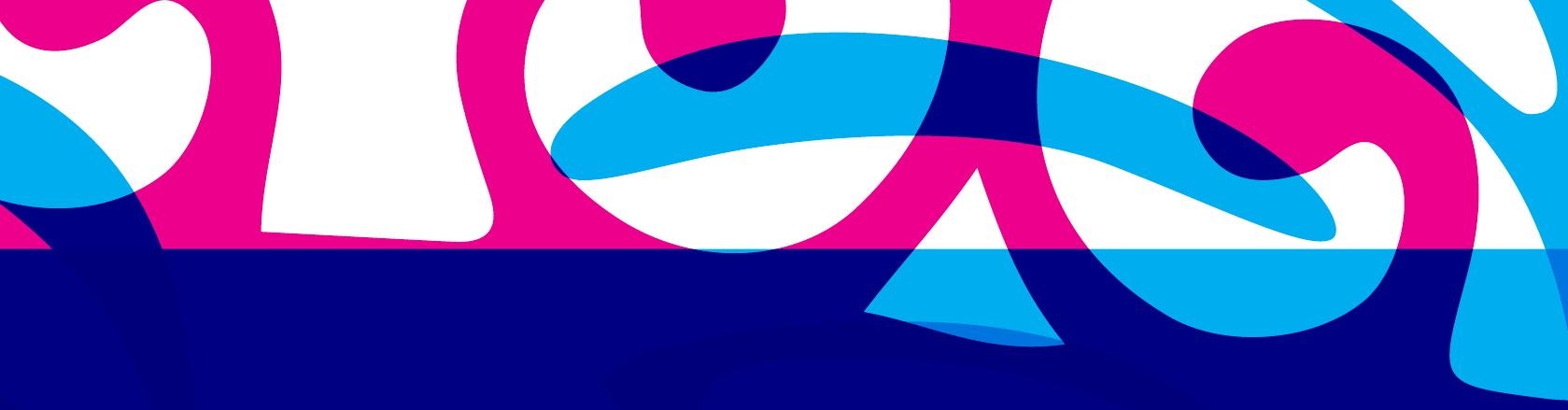




\section{CHAPTER 2}

DIRECT COMPARISON
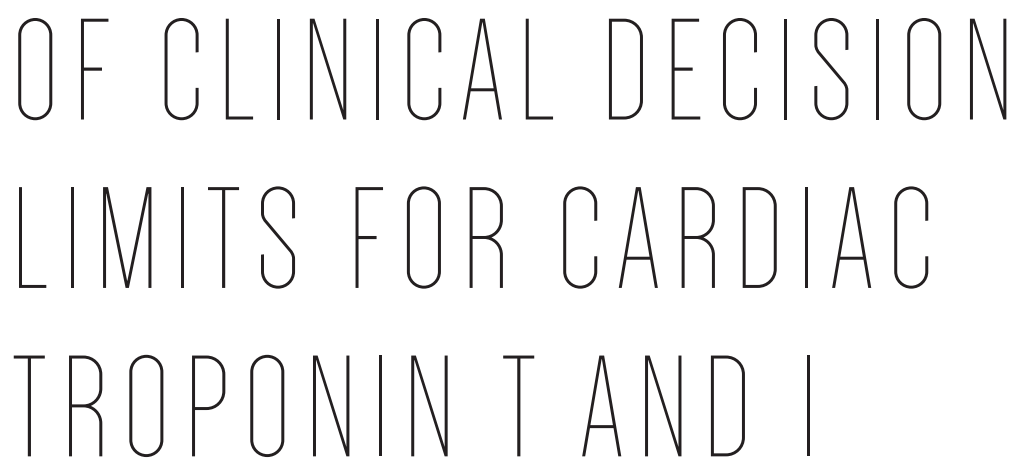

Dorien M Kimenai, Ronald MA Henry, Carla JH van der Kallen, Pieter C Dagnelie, Miranda T Schram, Coen DA Stehouwer, Jeroen DE van Süjlen, Marijke Niens, Otto Bekers, Simone JS Sep, Marja P van Dieijen-Visser, Steven JR Meex

Heart 2016; $102(8): 610-616$ 


\section{ABSTRACT}

\section{> OBJECTIVE}

The $99^{\text {th }}$ percentile upper reference limit of high-sensitivity cardiac troponin (hscTn) from a healthy reference population is used for diagnosing acute myocardial infarction (AMI). Accepted current thresholds of hs-cTnT (Roche) and hs-cTnI (Abbott) are $14 \mathrm{ng} / \mathrm{L}$ and $26 \mathrm{ng} / \mathrm{L}$, respectively. Since thresholds for hs-cTnT and hscTnI were derived from different reference cohorts it is unclear whether they are biologically equivalent. We directly assessed sex- and age-specific $99^{\text {th }}$ percentile upper reference limits of hs-cTnT and hs-cTnI in a single reference cohort, to investigate whether current divergent thresholds of hs-cTnT and hs-cTnI stem from intrinsic assay differences, or reflect cohort variation.

\section{> METHODS}

A healthy reference population was derived from a population based cohort (The Maastricht Study: $\mathrm{n}=3451$; age: 40 - 75 years). Individuals with diabetes mellitus, a history of cardiovascular disease, cardiac ischaemia on electrocardiography, $\mathrm{N}$-terminal pro-brain natriuretic peptide > $125 \mathrm{ng} / \mathrm{L}$, or estimated Glomerular Filtration Rate $<60 \mathrm{~mL} /$ minute $/ 1.73 \mathrm{~m}^{2}$ were excluded. Nonparametric analyses were performed to assess $99^{\text {th }}$ percentile upper reference limits.

\section{, RESULTS}

1540 individuals were included in the healthy reference population (age $57 \pm 8$ years, $52.4 \%$ women). Overall $99^{\text {th }}$ percentile upper reference limits of hs-cTnT and hs-cTnI were $15 \mathrm{ng} / \mathrm{L}$ and $13 \mathrm{ng} / \mathrm{L}$, respectively. Upper reference limits were higher in men than women (hs-cTnT: 16 versus $12 \mathrm{ng} / \mathrm{L}$ ), (hs-cTnI: 20 versus $11 \mathrm{ng} / \mathrm{L}$ ), and increased with age.

\section{> CONCLUSIONS}

Direct comparison reveals numerically similar thresholds for hs-cTnT and hs-cTnI assays. This finding is in line with recently reported underdiagnosis of AMI with the current decision limit of $26 \mathrm{ng} / \mathrm{L}$ for hs-cTnI, especially among women. Downward adjustment of the hs-cTnI threshold, differentiated for sex, would equalize clinical decision limits for both assays, and may prevent further underdiagnosis of AMI. 


\section{INTRODUCTION}

Cardiac troponin is the preferred biomarker for the diagnosis of acute myocardial infarction (AMI). ${ }^{1,2}$ The definition of AMI requires a significant "rise and/or fall" of high-sensitivity cardiac troponin (hs-cTn) between serial measurements with at least one value above the $99^{\text {th }}$ percentile upper reference limits of hs-cTn from a healthy reference population. ${ }^{2}$ The consensus requires that the $99^{\text {th }}$ percentile upper reference limit of hs-cTn should not be lower than the total imprecision, as described by $10 \%$ coefficient of variation $(10 \% \mathrm{CV}) .{ }^{1}$

There are two clinical troponin assays that meet all high-sensitivity criteria: the Roche high-sensitivity cardiac troponin T (hs-cTnT) assay, and the Abbott Architect high-sensitivity cardiac troponin I (hs-cTnI) assay. ${ }^{3}$ The presently recommended $99^{\text {th }}$ percentile upper reference limit for hs-cTnI is almost twice as high as that for hs-cTnT (26 ng/L versus $14 \mathrm{ng} / \mathrm{L}$, respectively, package inserts).

Since troponin $\mathrm{T}$ and I are different molecules, it is not surprising that they have different upper reference limits. More intriguing however, is the observation that the median troponin value of a healthy reference population is lower for hs-cTnI than hs-cTnT, which is opposite to what one would expect from the $99^{\text {th }}$ percentile values of both assays. ${ }^{4}$

The causes of divergent $99^{\text {th }}$ percentile threshold values between troponin $\mathrm{T}$ and I, and opposite effects on the median values of both assays, are unknown. A first possibility is that current upper reference limits for hs-cTnT and hs-cTnI were derived from different reference cohorts, and it is well established that different selection and screening procedures substantially affect the composition of presumably "healthy" reference cohorts. ${ }^{5-7}$ Previous studies recognized this issue and several approaches were developed for defining a healthy reference population. ${ }^{3,7-9}$ Recently, a standardized selection approach is reported for establishing accurate $99^{\text {th }}$ percentile values of cardiac troponin $\mathrm{T}$ and $\mathrm{I} .^{10}$ Second, intrinsic assay differences may play a role, in particular the frequency and distribution of extreme values, where differences across assays can profoundly impact the robustness of the $99^{\text {th }}$ percentile estimation. Third, and of high clinical importance, sex and age differences across reference cohorts may have contributed to substantial heterogeneity. ${ }^{6,11-18}$

The present study was designed to directly compare sex- and age-specific $99^{\text {th }}$ percentile upper reference limits for hs-cTnT and hs-cTnI, in relation to their median value, in a single, large, well-phenotyped healthy reference population. This approach allows to distinguish between cohort variation and intrinsic assay differences as factors that may contribute to divergent $99^{\text {th }}$ percentile upper reference limits, and apparently opposite effects on the median values. 


\section{METHODS}

\section{i STUDY DESIGN AND POPULATIONS}

In this study, we used data from The Maastricht Study, an ongoing observational prospective population-based cohort study. The rationale and methodology have been described previously. ${ }^{19}$ In brief, The Maastricht Study focuses on the aetiology, pathophysiology, complications and comorbidities of type 2 diabetes mellitus and is characterized by an extensive phenotyping approach. All participants gave written informed consent. The study was conducted according to the principles of the Declaration of Helsinki. ${ }^{20}$ Further details regarding The Maastricht Study cohort are provided in supplementary appendix. The healthy reference cohort was derived from The Maastricht Study cohort $(\mathrm{n}=3451)$ and defined as described in the supplemental data.

\section{, BIOMARKER MEASUREMENTS}

Morning blood samples were obtained from all individuals. Samples were centrifuged according to the manufacturer's instructions and serum samples were stored at $-80^{\circ} \mathrm{C}$ in aliquots. The storage time ranged from $1-4$ years. Prior to measurement, samples were thawed at room temperature, mixed and centrifuged at $2500 \mathrm{x} \mathrm{g}$ for two minutes. Serum cTnT was measured, using the Roche Cobase6o1 hs-cTnT assay (Roche) on the Cobas6ooo analyser. This method has a limit of blank (LoB) of $3 \mathrm{ng} / \mathrm{L}$, limit of detection (LoD) of $5 \mathrm{ng} / \mathrm{L}$, and $10 \% \mathrm{CV}$ of $13 \mathrm{ng} / \mathrm{L}$ (package insert). Hs-cTnI was measured after an additional freeze-thaw step. Potential preanalytical bias was investigated by reassessment of $3 \%$ of the hs-cTnI measurements. Reassessed values correlated strongly with the original data (Pearson's correlation coefficient: 0.998 , 95\% CI $0.996-0.999, \mathrm{P}<$ o.00o1; intercept: -0.81, 95\% CI -1.71 - 0.09), making pre-analytical bias unlikely. Serum cTnI was measured with ARCHITECT i20ooSR STAT hs-cTnI assay (Abbott). According to the package insert, LoB ranged between 0.7 and 1.3 ng/L and LoD ranged between 1.1 and 1.9 ng/L. The hs-cTnI assay has a 10\% CV of $4.7 \mathrm{ng} / \mathrm{L}$ (package insert).

\section{- STATISTICAL ANALYSIS}

Continuous variables are expressed as mean and standard deviation (SD), or median and interquartile range (IQR) when not normally distributed. Categorical data are reported as number and percentage.

The correlation between log-transformed hs-cTnT and log-transformed hs-cTnI was assessed by Pearson's correlation test. The relationship between hs-cTn and age was examined with Spearman's correlation test.

Non-parametric analyses were performed to determine $99^{\text {th }}$ percentile upper reference limits of hs-cTnT and hs-cTnI, stratified by sex and age. Bias corrected and accelerated bootstrapped percentile method (resampling with replacement: 5,000 bootstrap replicates) was used to determine $95 \%$ confidence intervals ( $95 \%$ 
CIs) for the stratified $99^{\text {th }}$ percentile upper reference limits. Uncorrected and outlier-adjusted $99^{\text {th }}$ percentiles according to Dixon's outlier detection method were calculated. Briefly, Dixon's outlier method is based on D/R ratio whereby D is the absolute difference between the most extreme value and the preceding value and $\mathrm{R}$ is the range of the values (maximum - minimum). ${ }^{21}$ If the $D / R$ ratio is $>1 / 3$, the extreme value is considered an outlier and excluded from analyses. Dixon's method further dictates that a group of extreme values can be evaluated as a whole, by testing the least extreme value of this group according to the $\mathrm{D} / \mathrm{R}$ ratio criterion. If this is considered an outlier $(D / R$ ratio $>1 / 3)$, also the more extreme values of this group are excluded.

Sensitivity analyses with the non-parametric Tukey's outlier detection method were performed to verify robustness of the calculated $99^{\text {th }}$ percentile upper reference limits, independent of the outlier detection method applied. The Tukey method is based on $25^{\text {th }}$ quartile $\left(\mathrm{Q}_{1}\right), 75^{\text {th }}$ quartile $\left(\mathrm{Q}_{3}\right)$ and IQR. ${ }^{22}$ For the Tukey method the hs-cTn values were Box-Cox transformed. By means of these values lower and upper fences were defined: lower fence $=\mathrm{Q}_{1}-3$ (IQR), upper fence $=\mathrm{Q}_{3}+3$ (IQR). Values above the upper fence and under the lower fence were defined as outliers and excluded from analyses.

Finally, $97.5^{\text {th }}$ percentile upper reference limits were calculated for both troponin assays. This analysis is an even more stringent approach than the classical outlier detection methods against the influence of extreme values in the right tail of the troponin distribution. Hence, differences in the dispersion of values in the right tail of the troponin distributions, and their effect on the upper reference value of both assays, can be identified. Statistical tests were performed using SPSS V.20 (SPSS Inc., Chicago, Illinois, USA) and STATA V.13 (Stata Corp., College Station, Texas, USA).

\section{RESULTS}

\section{> DETECTION AND DISPERSION OF HS-CTNT AND HS-CTNI IN THE HEALTHY REFERENCE COHORT} A total of 1540 individuals from The Maastricht Study cohort were included in the healthy reference population (Figure 2.1). Baseline characteristics of the entire reference population and subpopulations are shown in Table 2.1.

The proportion of subjects with a measurable troponin $\mathrm{T}$ level ( $\mathrm{LoD} \geq 5 \mathrm{ng} / \mathrm{L}$ ) in this healthy reference population was $43.4 \%$, in line with previously reported findings $(24 \%-58 \%))^{8,12-15}$ The proportion with detectable troponin I concentrations ranged between $43.1 \%$ and $79.1 \%$, depending on the LoD that is employed (troponin I has an LoD range of $1.1-1.9 \mathrm{ng} / \mathrm{L})$. Previous studies applied a fixed LoD of $1.9 \mathrm{ng} / \mathrm{L}$ and reported a range of detectable troponin I concentrations between $64 \%$ and $81 \%^{6,16}$ Percentages of measurable troponin levels stratified by sex are shown in Supplemental Table 2.1. 
The proportion of subjects with measurable concentrations with both assays ranged between $29.1 \%$ and $41.3 \%$, depending the troponin I LoD applied (Supplemental Table 2.1). The median (IQR) was $5(3.4-6.3) \mathrm{ng} / \mathrm{L}$ for hs-cTnT and $2(1.1-2.5) \mathrm{ng} / \mathrm{L}$ for hs-cTnI. The dispersion of values directly around the median was smaller for hs-cTnI than for hs-cTnT, however, both the frequency and the absolute value of extreme values were higher for hs-cTnI than hs-cTnT (Figure 2.2).

To verify that heterophilic antibodies did not underlie the relatively extended right tail of the troponin I distribution, all samples of The Maastricht Study cohort with a hs$\mathrm{cTnI}>11 \mathrm{ng} / \mathrm{L}(\mathrm{n}=102)$ were reassessed in a dilution series $(1: 2 ; 1: 4)$. No evidence for assay interference was found in any of these samples (data not shown), suggesting that the values representing the stretched right tail of the hs-cTnI distribution are analytically correct. The Pearson's correlation coefficient between log-transformed hs-cTnT and log-transformed hs-cTnI was 0.545 (95\% CI $0.509-0.579, \mathrm{P}<0.0001$ ) (Figure 2.3).

Excluded, variables were not mutually exclusive $(n=1,8 \mid 3)$

$-\mathrm{T} 2 \mathrm{DM}(\mathrm{n}=975)$

$-\operatorname{TIDM}(n=37)$

- Other DM $(n=4)$

- Known history of CVD ( $n=558)$

- Prior cardiac ischemia on ECG $(n=627)$

- NT-proBNP > $125 \mathrm{ng} / \mathrm{L}(\mathrm{n}=503)$

- eGFR $<60 \mathrm{~mL} /$ minute $/ .73 \mathrm{~m}^{2}(\mathrm{n}=147)$

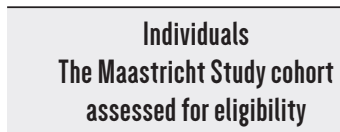

assessed for eligibility $(n=3,451)$

Missing data, variables were not mutually exclusive $(n=98)$

- No hs-cTnT/hs-cTnl levels ( $n=18$ )

- Unknown history of CVD ( $n=4 I)$

- No ECG data available $(n=40)$
Detection outliers - Dixon $(n=5)$

- hs-cTnT $(n=2)$

- hs-cTnl $(n=3)$
Reference population $(n=1,540)$

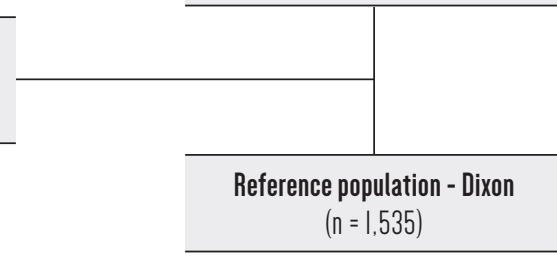




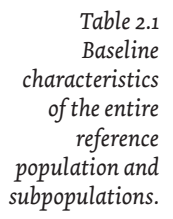

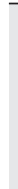

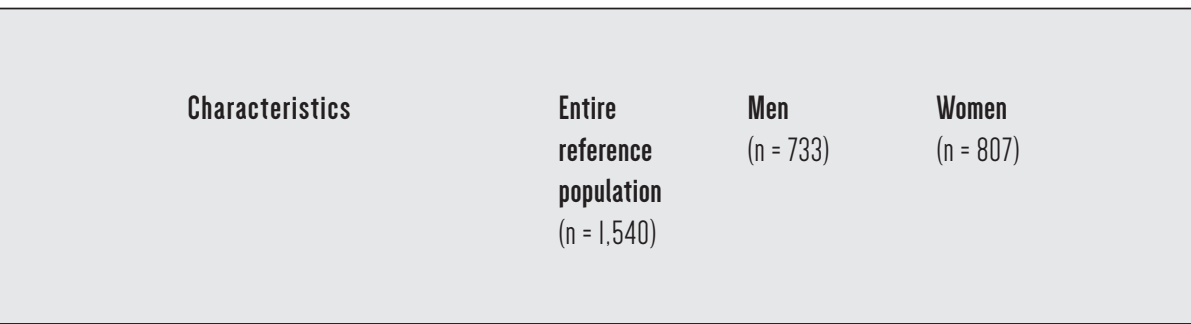

\begin{tabular}{|c|c|c|c|}
\hline Age (years) & $57(8)$ & $58(8)$ & $57(8)$ \\
\hline Sex (male) & $733(47.6 \%)$ & $733(100 \%)$ & $0(0 \%)$ \\
\hline Weight (kg) & $75(66-85)$ & $83(76-90)$ & $67(61-75)$ \\
\hline Height (cm) & I7| (9) & $178(6)$ & $165(6)$ \\
\hline $\operatorname{BMI}\left(\mathrm{kg} / \mathrm{m}^{2}\right)^{\mathrm{a}}$ & $26(23-28)$ & $26(24-28)$ & $25(23-27)$ \\
\hline Hypertension (yes) & $604(39.3 \%)$ & $356(48.7 \%)$ & $248(30.7 \%)$ \\
\hline \multicolumn{4}{|l|}{ Smoking ${ }^{\mathrm{a}}$} \\
\hline Never & $602(39.1 \%)$ & $281(38.4 \%)$ & $321(39.8 \%)$ \\
\hline Former & $737(47.9 \%)$ & $350(47.8 \%)$ & $387(48.0 \%)$ \\
\hline Current & $200(13.0 \%)$ & |O| (|3.8\%) & $99(12.3 \%)$ \\
\hline BP lowering medication (yes) ${ }^{\mathrm{a}}$ & $30 \mid(\mid 9.6 \%)$ & $169(23.1 \%)$ & $132(16.4 \%)$ \\
\hline Lipid lowering medication (yes)a & $226(14.7 \%)$ & |3| (17.9\%) & $95(11.8 \%)$ \\
\hline Total cholesterol (mmol/L) & $5.6(1.0)$ & $5.5(1.0)$ & $5.8(1.0)$ \\
\hline HDL-cholesterol (mmol/L) & $1.6(1.3-1.9)$ & $1.4(1.2-1.6)$ & $1.8(1.5-2.1)$ \\
\hline LDL-cholesterol (mmol/L) & $3.4(0.9)$ & $3.4(0.4)$ & $3.4(0.9)$ \\
\hline Triglycerides (mmol/L) & I.I (0.8-1.5) & $1.2(0.9-1.7)$ & I.I (0.8-1.5) \\
\hline eGFR $\left(\mathrm{mL} / \mathrm{minute} / \mathrm{l} .73 \mathrm{~m}^{2}\right)$ & 9I (I2) & $91(\mid 2)$ & 9I (12) \\
\hline BP, Systolic (mmHg) & $|3|(17)$ & $137(15)$ & $127(16)$ \\
\hline BP, Diastolic $(\mathrm{mmHg})^{\mathrm{a}}$ & $76(10)$ & $79(10)$ & $73(9)$ \\
\hline
\end{tabular}




$\begin{array}{llllll}\text { Men, } & \text { Men, } & \text { Men, } & \text { Women, } & \text { Women, } & \text { Women, } \\ 40-49 & 50-64 & 65-75 & 40-49 & 50-64 & 65-75 \\ \text { years } & \text { years } & \text { years } & \text { years } & \text { years } & \text { years } \\ (n=120) & (n=443) & (n=170) & (n=163) & (n=503) & (n=141)\end{array}$

\begin{tabular}{|c|c|c|c|c|c|c|}
\hline 45 (3) & $58(4)$ & $68(3)$ & $45(3)$ & $57(4)$ & $68(3)$ & \\
\hline $120(100 \%)$ & $443(100 \%)$ & $170(100 \%)$ & $0(0 \%)$ & $0(0 \%)$ & $0(0 \%)$ & \\
\hline $84(77-91)$ & $83(77-92)$ & $80(74-86)$ & 67 (62-78) & $67(61-76)$ & $67(61-74)$ & \\
\hline $179(6)$ & $179(6)$ & $176(6)$ & $167(6)$ & $165(6)$ & $163(5)$ & \\
\hline $26(24-28)$ & $26(24-29)$ & $26(24-27)$ & $25(22-28)$ & $25(22-27)$ & $25(23-27)$ & \\
\hline 34 (28.3\%) & $218(49.2 \%)$ & 104 (61.9\%) & $26(16.0 \%)$ & $157(31.2 \%)$ & 65 (46.1\%) & \\
\hline $\begin{array}{l}62(51.7 \%) \\
38(31.7 \%) \\
20(16.7 \%)\end{array}$ & $\begin{array}{l}168(37.9 \%) \\
207(46.7 \%) \\
68(15.3 \%)\end{array}$ & $\begin{array}{l}51(30.2 \%) \\
105(62.1 \%) \\
13(7.7 \%)\end{array}$ & $\begin{array}{l}86(52.8 \%) \\
46(28.2 \%) \\
31(19.0 \%)\end{array}$ & $\begin{array}{l}178(35.4 \%) \\
266(52.9 \%) \\
59(11.7 \%)\end{array}$ & $\begin{array}{l}57(40.4 \%) \\
75(53.2 \%) \\
9(6.4 \%)\end{array}$ & \\
\hline $6(5.0 \%)$ & 105 (23.7\%) & $58(34.3 \%)$ & $15(9.2 \%)$ & $86(17.1 \%)$ & 31 (22.0\%) & \\
\hline $9(7.5 \%)$ & $80(18.1 \%)$ & 42 (24.9\%) & $5(3.1 \%)$ & $54(10.7 \%)$ & $36(25.5 \%)$ & \\
\hline $5.4(1.0)$ & $5.5(1.0)$ & $5.5(1.0)$ & $5.2(0.9)$ & $5.9(1.0)$ & $5.9(1.0)$ & \\
\hline $1.3(1.0-1.5)$ & $1.4(1.2-1.6)$ & $1.5(1.3-1.7)$ & $1.7(1.4-1.9)$ & $1.8(1.5-2.1)$ & $1.8(1.5-2.1)$ & \\
\hline $3.5(1.0)$ & $3.4(0.9)$ & $3.4(0.9)$ & $3.1(0.8)$ & $3.5(1.0)$ & $3.5(0.9)$ & \\
\hline $1.2(0.9-1.6)$ & $1.2(0.9-1.7)$ & I.I (0.9-1.6) & $1.0(0.7-1.3)$ & I.I (0.8-1.5) & I.I (0.9-1.5) & $\begin{array}{l}\text { Continuous } \\
\text { variables are }\end{array}$ \\
\hline 100 (II) & $91(I I)$ & $85(12)$ & $100(12)$ & 9I (II) & $82(10)$ & $\begin{array}{l}\text { mease of normal } \\
\text { distribution, }\end{array}$ \\
\hline$|3|(\mid 3)$ & $137(15)$ & $140(16)$ & $\| 9(13)$ & $127(16)$ & $132(19)$ & $\begin{array}{l}\text { reported as } \\
\text { median (IQR). }\end{array}$ \\
\hline $79(10)$ & $80(10)$ & $77(9)$ & $72(9)$ & $74(9)$ & $72(9)$ & $\begin{array}{l}\text { Categorical data } \\
\text { are reported } \\
\text { as } n(\%) .\end{array}$ \\
\hline
\end{tabular}


Figure 2.2

Distribution of hs-cTnT (A) and hs-cTnI (B) of healthy reference population.

Figure 2.3 Scatterplot of the relationship between log-transformed hs-cTnT and log-transformed hs-cTnI ( $\beta=0.983$ (95\% CI $0.908-1.059$ ), $\left.R^{2}=0.297\right)$.
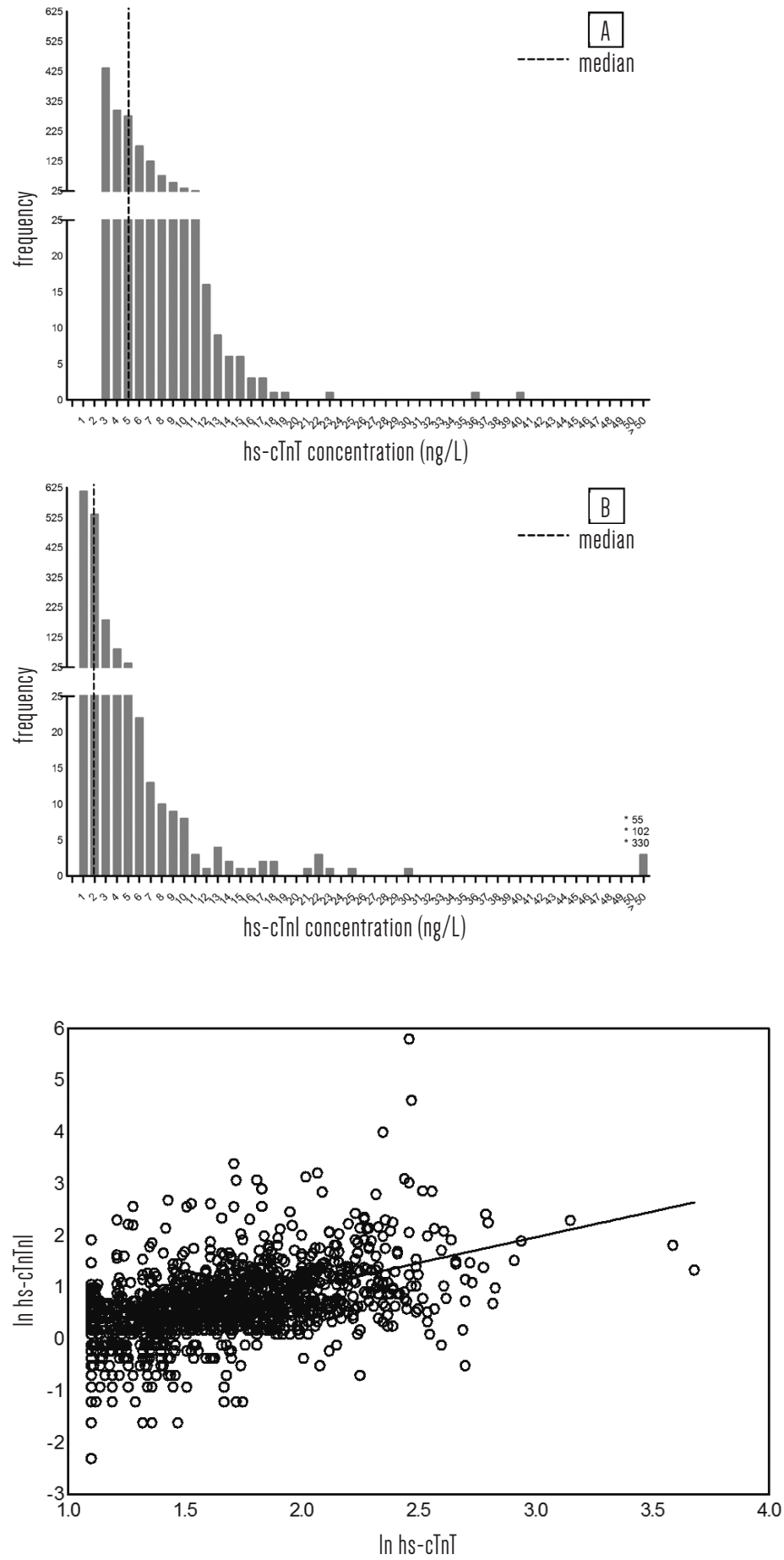


\section{> THE 99TH PERCENTILE UPPER REFERENCE LIMITS OF HS-CTNT AND HS-CTNI}

By direct comparison, uncorrected $99^{\text {th }}$ percentile upper reference limits of hs-cTnT and hs-cTnI assays were in the same range with overlapping 95\% CIs: hs-cTnT, 15 (95\% CI 14 - 16) ng/L vs hs-cTnI, 16 (95\% CI 12 - 21) ng/L (Table 2.2). Two hs-cTnT values and three hs-cTnI values were considered outliers according to Dixon's outlier detection method, leaving 1,535 individuals for refined outlier-adjusted analyses. The calculated $99^{\text {th }}$ percentile threshold of hs-cTnT was unaffected after outlier removal (Table 2.2). Unlike hs-cTnT, outlier-correction decreased the overall hs-cTnI upper reference limit with 3 ng/L (hs-cTnI, uncorrected: $16 \mathrm{ng} / \mathrm{L}$; hs-cTnI, outlier-adjusted: $13 \mathrm{ng} / \mathrm{L}$ ) (Table 2.2), which further equalized the $99^{\text {th }}$ percentile upper reference limits of hs-cTnT and hs-cTnI.

To verify that the $99^{\text {th }}$ percentile upper reference limits were robust and independent of the outlier detection method applied, sensitivity analysis was performed to compare results from Dixon's and Tukey's outlier detection methods. Two hs-cTnT values were outliers according to Dixon, no hs-cTnT measurements were considered outliers according to Tukey. Three hs-cTnI values were outliers according to Dixon's method and 10 hs-cTnI values were outliers according to Tukey's method. Calculated $99^{\text {th }} \mathrm{hs}$-cTn percentile upper reference limits were largely independent of the outlier detection method applied (Figure 2.4).

\begin{tabular}{|c|c|c|c|}
\hline & $\mathrm{n}$ & hs-cTnT, ng/L (95\% Cl) & hs-cTnl, ng/L (95\% Cl) \\
\hline \multicolumn{4}{|l|}{ Uncorrected $99^{\text {th }}$ percentile } \\
\hline Reference population & 1,540 & $15(14-16)$ & $16(12-21)$ \\
\hline \multicolumn{4}{|l|}{ Stratified by sex } \\
\hline Men & 733 & $16(15-18)$ & $22(16-46)$ \\
\hline Women & 807 & $12(10-15)$ & $\|(8-13)$ \\
\hline \multicolumn{4}{|l|}{ Stratified by sex, age } \\
\hline Men, 40 - 49 years & 120 & $16(10-17)$ & $13(5-15)$ \\
\hline Men, 50 - 64 years & 443 & $14(13-16)$ & $23(16-55)$ \\
\hline Men, 65 - 75 years & 170 & $28(19-40)$ & $113(17-330)$ \\
\hline Women, 40 - 49 years & 163 & $12(7-16)$ & $12(10-14)$ \\
\hline Women, 50 - 64 years & 503 & $12(9-15)$ & $9(6-14)$ \\
\hline Women, 65 - 75 years & $|4|$ & $27(12-36)$ & $13(10-13)$ \\
\hline \multicolumn{4}{|c|}{ Outlier-adjusted $99^{\text {th }}$ percentile } \\
\hline Reference population & 1,535 & $15(13-16)$ & |3 (|| - 18) \\
\hline \multicolumn{4}{|l|}{ Stratified by sex } \\
\hline Men & 729 & $16(15-17)$ & $20(14-22)$ \\
\hline Women & 806 & $12(10-14)$ & $\|(8-13)$ \\
\hline \multicolumn{4}{|l|}{ Stratified by sex, age } \\
\hline Men, 40 - 49 years & 120 & $16(10-17)$ & $13(5-15)$ \\
\hline Men, 50 - 64 years & 441 & $14(13-16)$ & $22(13-23)$ \\
\hline Men, 65 - 75 years & 168 & $20(17-23)$ & $20(13-25)$ \\
\hline Women, 40 - 49 years & 163 & $12(7-16)$ & $12(10-14)$ \\
\hline Women, 50 - 64 years & 503 & $12(9-15)$ & $9(6-14)$ \\
\hline Women, 65 - 75 years & 140 & $\mid 3(||-\mid 4)$ & $13(10-13)$ \\
\hline
\end{tabular}

Table 2.2 The $99^{\text {th }}$ percentile upper reference limits ( $n g / L, 95 \%$ CI) forhs-cTnT and hs-cTnI. 


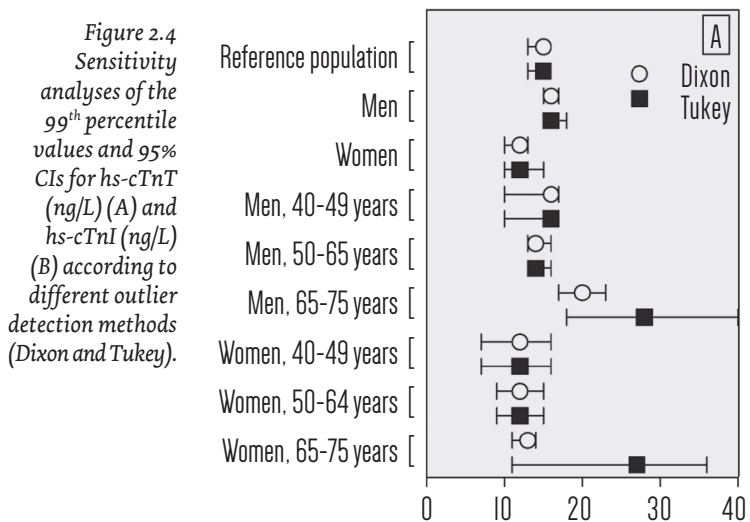

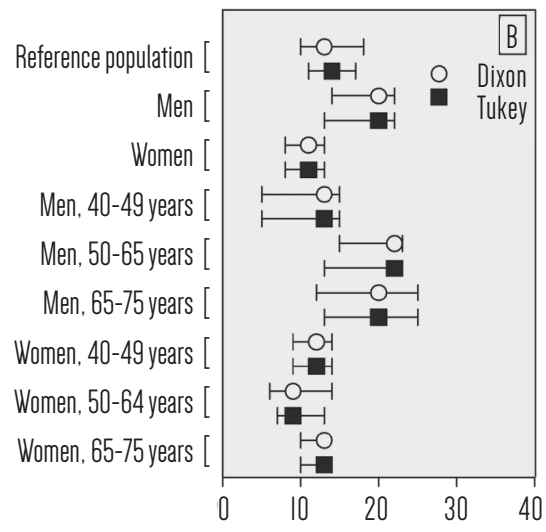

\section{$>$ THE 97.5TH PERCENTILE UPPER REFERENCE LIMITS OF HS-CTNT AND HS-CTNI}

The troponin I distribution is characterized by a more extended right tail than the troponin $\mathrm{T}$ distribution, in particular when compared to the median value of both distributions (hs-cTnT: $5 \mathrm{ng} / \mathrm{L}$, hs-cTnI: $2 \mathrm{ng} / \mathrm{L}$; Figure 2.2, compare A to B). This suggests that variation in a very small proportion of the reference cohort (1-2\%) strongly influences calculated upper reference limits of troponin I, whereas troponin $\mathrm{T}$ is more robust in terms of variation in the extremity of the right tail of the distribution.

To quantitate the effect of this phenomenon on calculated upper reference limits, $97.5^{\text {th }}$ percentile upper reference limits were assessed for both assays. The $97.5^{\text {th }}$ percentile not only eliminates the undesirable effect of outliers on the upper reference limit, but also reduces the disproportional influence of widely dispersed tails, which are often not representative for the vast majority of measurements in a healthy reference cohort. The calculated $97.5^{\text {th }}$ percentile upper reference limits were $12 \mathrm{ng} / \mathrm{L}$ for hs-cTnT and $9 \mathrm{ng} / \mathrm{L}$ for hs-cTnI, a decrease of respectively $\sim 20 \%$ versus $\sim 60 \%$ compared with the uncorrected $99^{\text {th }}$ percentile upper reference limits of both assays (Table 2.3).

The much larger numerical difference between the $99^{\text {th }}$ and $97.5^{\text {th }}$ percentile for troponin I compared with troponin $\mathrm{T}$, confirm the much wider dispersed tails of troponin I, which makes the $99^{\text {th }}$ percentile upper reference limit of troponin I less robust, and more subject to variation and outliers.

\section{> SEX-SPECIFIC AND AGE-SPECIFIC UPPER REFERENCE LIMITS OF HS-CTNT AND HS-CTNI}

For both assays, $99^{\text {th }}$ percentile upper reference limits were higher in men than women. For hs-cTnT the $99^{\text {th }}$ percentile was $16 \mathrm{ng} / \mathrm{L}$ for men and $12 \mathrm{ng} / \mathrm{L}$ for women. For hs-cTnI the $99^{\text {th }}$ percentile was $20 \mathrm{ng} / \mathrm{L}$ for men and $11 \mathrm{ng} / \mathrm{L}$ for women 


\begin{tabular}{|c|c|c|c|c|}
\hline & n & hs-cTnT, ng/L (95\% Cl) & hs-cTnl, ng/L (95\% CI) & \multirow{12}{*}{$\begin{array}{l}\text { Table 2.3 } \\
\text { The } 97.5^{\text {th }} \\
\text { percentile upper } \\
\text { reference limits } \\
\text { (ng/L, } 95 \% \text { CI) } \\
\text { for hs-cTnT } \\
\text { and hs-cTnI. }\end{array}$} \\
\hline Reference population & 1,540 & $12(\mid 1-13)$ & $9(8-10)$ & \\
\hline \multicolumn{4}{|l|}{ Stratified by sex } & \\
\hline Men & 733 & $14(12-15)$ & II (9- 18) & \\
\hline Women & 807 & $9(8-10)$ & $6(5-9)$ & \\
\hline \multicolumn{4}{|l|}{ Stratified by sex, age } & \\
\hline Men, 40 - 49 years & 120 & $11(8-17)$ & $5(4-15)$ & \\
\hline Men, 50 - 64 years & 443 & $13(12-14)$ & $13(9-22)$ & \\
\hline Men, 65 - 75 years & 170 & $18(15-23)$ & $16(8-50)$ & \\
\hline Women, 40 - 49 years & 163 & $7(6-16)$ & $10(3-11)$ & \\
\hline Women, 50 - 64 years & 503 & $9(8-11)$ & $5(5-8)$ & \\
\hline Women, 65 - 75 years & $|4|$ & $12(9-28)$ & $9(5-13)$ & \\
\hline
\end{tabular}

(Table 2.2). The Spearman's correlation coefficient between hs-cTn and age was 0.398 (95\% CI $0.354-0.440, \mathrm{P}<0.0001$ ) for hs-cTnT and 0.292 (95\% CI $0.244-0.338$, $\mathrm{P}<0.0001)$ for hs-cTnI. The $99^{\text {th }}$ percentile upper reference limits progressively increased per age stratum, in particular for the hs-cTnT assay. Similar to the overall $99^{\text {th }}$ percentile upper reference limits, sex- and age-specific estimates are less robust for troponin I than troponin $\mathrm{T}$ (see CIs Table 2.2).

\section{DISCUSSION}

In a direct comparison of troponin $\mathrm{T}$ and $\mathrm{I}$ upper reference limits, the present study reveals remarkably similar $99^{\text {th }}$ percentile upper reference limits of $15 \mathrm{ng} / \mathrm{L}$ and $13 \mathrm{ng} / \mathrm{L}$ for the hs-cTnT (Roche) and hs-cTnI (Abbott) assay, respectively. The $99^{\text {th }}$ percentile upper reference limit of $13 \mathrm{ng} / \mathrm{L}$ for hs-cTnI contrasts with the currently employed decision limit of $26 \mathrm{ng} / \mathrm{L}$. One previous study performed a direct comparison between troponin $\mathrm{T}$ and I but focused mainly on the effect of population selection on the $99^{\text {th }}$ percentile upper reference limits. ${ }^{7}$

An important strength of this study is that the $99^{\text {th }}$ percentile upper reference limits of hs-cTnT and hs-cTnI were obtained from a single healthy reference population. Although proposed hs-cTnT thresholds are relatively reproducible in different "healthy" reference populations, it seems that hs-cTnI thresholds are less robust across studies, and decrease substantially in reference populations that applied more stringent selection criteria..$^{5}$ Our approach eliminated cohort variation as an uncontrollable source of heterogeneity, and enabled the estimation of biologically identical clinical decision limits for troponin $\mathrm{T}$ and $\mathrm{I}$. Current non-bioequivalent $99^{\text {th }}$ percentile upper reference limits of hs-cTnT and hs-cTnI are a worrying source of misdiagnosis of AMI and harm to patients. ${ }^{23}$ The clinical importance of our key 
finding - a lower clinical decision limit for troponin I - is recently illustrated in a study by Wildi et al. ${ }^{23}$ They showed that one out of five AMI patients has an inconsistent diagnosis when using the hs-cTnT or hs-cTnI assays with currently approved upper reference limits of $14 \mathrm{ng} / \mathrm{L}$ and $26 \mathrm{ng} / \mathrm{L}$, respectively. This inconsistency was driven by a too stringent hs-cTnI clinical decision limit of $26 \mathrm{ng} / \mathrm{L}$, and led to underdiagnosis of AMI with the troponin I assay. ${ }^{23}$ Importantly, the percentage of inconsistent diagnoses could be halved by downwards adjustment of the hs-cTnI threshold to $9 \mathrm{ng} / \mathrm{L} .{ }^{23}$ The present study therefore corroborates and extends these observations, and strengthens the evidence of two studies who reported lower hs-cTnI thresholds than currently employed. ${ }^{6,7}$

Our in-depth exploration of the dispersion of measurements with both hs-cTn assays showed that the $99^{\text {th }}$ percentile upper reference limit of the hs-cTnT assay is less susceptible to variation and outliers in the extremity of the right tail of the distribution than the hs-cTnI assay. Importantly, this finding is robust and unrelated to the lower LoD of troponin I relative to troponin T: when a troponin I LoD threshold at the right side of the reported troponin I LoD range is employed (1.9 ng/L instead of $1.1 \mathrm{ng} / \mathrm{L}$ ), which brings the proportion of subjects with measurable concentrations to an identical percentage as the troponin $\mathrm{T}$ assay (43\%), the number of outliers remained similar. These intrinsic assay differences, along with cohort variation, may have contributed to rather inconsistent $99^{\text {th }}$ percentile upper reference limits for troponin I compared to troponin $\mathrm{T}$ across studies, ${ }^{5}$ and underlie current non-bioequivalent clinical decision limits for troponin $\mathrm{T}$ and $\mathrm{I}$.

Adequate selection and screening procedures are of great importance to compose a healthy reference cohort. Our study fulfilled the recent Sandoval criteria for defining a healthy reference population and therefrom sexspecific and age-specific upper reference limits of hs-cTnT and hs-cTnI assays were assessed. In line with previous studies $99^{\text {th }}$ percentile upper reference limits were sex-specific, with lower levels in women than men., ${ }^{4,6-8,11-18,24}$ This finding extends recent clinical data that the generally used "one size fits all" clinical decision limits are too high for women, leading to underdiagnosis of AMI. ${ }^{25}$ Sex-specific thresholds may tackle this clinical problem. The $99^{\text {th }}$ percentile threshold of hs-cTnT also increased with age, in particular in men $\geq 65$ years. Age-dependency of the hs-cTnT upper reference limit was also evident in women, though less pronounced in outlier-adjusted analyses. Troponin I revealed a similar trend of age-dependency, although not as clear as hs-cTnT. This is consistent with the lower correlation coefficients between hscTnI and age compared with hs-cTnT. However, the larger Cls confirm that the much wider dispersed tails of troponin I complicate robust assessment of the $99^{\text {th }}$ percentile for troponin I, in particular in stratified analyses with less individuals.

The requirement that clinically used thresholds should be >10\% CV merits consideration. The women-specific hs-cTnT threshold of $12 \mathrm{ng} / \mathrm{L}$ exceeds the $10 \%$ $\mathrm{CV}$ of $13 \mathrm{ng} / \mathrm{L}$. Especially for women $<65$ years it is with the current definition not possible to adjust the hs-cTnT threshold downwards, as the $99^{\text {th }}$ percentile upper 
reference limit would not meet the required precision. However, adjustment for the hs-cTnI threshold is possible, since the women-specific $99^{\text {th }}$ percentile upper reference limit of $11 \mathrm{ng} / \mathrm{L}>10 \% \mathrm{CV}$ of $5 \mathrm{ng} / \mathrm{L}$. The clinically relevance of sex-specific $99^{\text {th }}$ percentile upper reference limits of hs-cTnI, with in particular a single threshold for women, requires further urgent attention. The next step should be a multicentre randomised controlled trial investigating the performance of the adjusted sexspecific hs-cTnI thresholds. This should clarify whether sex-specific thresholds can effectively reduce the underdiagnosis of AMI.

Limitations of this study merit attention. 1) Individuals from The Maastricht Study were mainly of Caucasian origin and all individuals were between 40 and 75 years of age. Hence, $99^{\text {th }}$ percentile upper reference limits cannot be extrapolated to other ethnicities and age groups. 2) Men and women were not matched or adjusted for characteristics. Hence, we cannot formerly exclude the possibility that differences in upper reference limits are (in part) attributable to differences in patient case mix. 3) Imaging modalities such as cardiac echocardiography were not yet available in the current dataset. It is conceivable that inclusion of imaging modalities, although not formally recommended, would have resulted in even slightly lower upper reference limits. These limitations, however, do not affect the key finding of this study, being that $99^{\text {th }}$ percentile upper reference limits of hs-cTnI should be adjusted downwards to become more equivalent to the clinical decision limit that is used for hs-cTnT, and reduce underdiagnosis of AMI.

In conclusion, direct comparison from a single reference cohort reveals numerically similar $99^{\text {th }}$ percentile upper reference limits for hs-cTnT and hs-cTnI assays. Downwards adjustment of the $99^{\text {th }}$ percentile upper reference limit of hs-cTnI, differentiated for sex, would equalise clinical decision limits for both assays, and may prevent underestimation of AMI in clinical practice. Sex-specific clinical decision limits seems to be crucial to reduce underdiagnosis of AMI in women. 
REFERENCES

1. Thygesen K, Alpert JS, Jaffe AS, et al. Third universal definition of myocardial infarction. Journal of the American College of Cardiology 2012;60(16):1581-98.

2. Authors/Task Force M, Roffi M, Patrono C, et al. 2015 ESC Guidelines for the management of acute coronary syndromes in patients presenting without persistent ST-segment elevation: Task Force for the Management of Acute Coronary Syndromes in Patients Presenting without Persistent ST-Segment Elevation of the European Society of Cardiology (ESC). European heart journal 2016;37(3):267-315.

3. Apple FS, Collinson PO, Biomarkers ITFoCAoC. Analytical characteristics of high-sensitivity cardiac troponin assays. Clinical chemistry 2012;58(1):54-61.

4. Mingels A, Jacobs L, Michielsen E, et al. Reference population and marathon runner sera assessed by highly sensitive cardiac troponin $\mathrm{T}$ and commercial cardiac troponin $\mathrm{T}$ and $\mathrm{I}$ assays. Clinical chemistry 2009;55(1):101-8.

5. Cardinaels EP, Mingels AM, Jacobs LH, et al. A comprehensive review of upper reference limits reported for (high-)sensitivity cardiac troponin assays: the challenges that lie ahead. Clinical chemistry and laboratory medicine : CCLM / FESCC 2012;50(5):791-806.

6. Krintus M, Kozinski M, Boudry P, et al. Defining normality in a European multinational cohort: Critical factors influencing the $99^{\text {th }}$ percentile upper reference limit for high sensitivity cardiac troponin I. International journal of cardiology 2015;187:256-63.

7. Koerbin G, Abhayaratna WP, Potter JM, et al. Effect of population selection on $99^{\text {th }}$ percentile values for a high sensitivity cardiac troponin I and $\mathrm{T}$ assays. Clinical biochemistry 2013;46(16-17):1636-43.

8. Collinson PO, Heung YM, Gaze D, et al. Influence of population selection on the $99^{\text {th }}$ percentile reference value for cardiac troponin assays. Clinical chemistry 2012;58(1):219-25.

9. McKie PM, Heublein DM, Scott CG, et al. Defining high-sensitivity cardiac troponin concentrations in the community. Clinical chemistry 2013;59(7):1099-107.

10. Sandoval Y, Apple FS. The global need to define normality: the $99^{\text {th }}$ percentile value of cardiac troponin. Clinical chemistry 2014;60(3):455-62.

11. Giannitsis E, Kurz K, Hallermayer K, et al. Analytical validation of a high-sensitivity cardiac troponin T assay. Clinical chemistry 2010;56(2):254-61.

12. Saenger AK, Beyrau R, Braun S, et al. Multicenter analytical evaluation of a high-sensitivity troponin $\mathrm{T}$ assay. Clinica chimica acta; international journal of clinical chemistry 2011;412 $(9-10): 748-54$. 
13. Koerbin G, Tate JR, Hickman PE. Analytical characteristics of the Roche highly sensitive troponin $\mathrm{T}$ assay and its application to a cardio-healthy population. Annals of clinical biochemistry 2010;47(Pt 6):524-8.

14. Gaggin HK, Dang PV, Do LD, et al. Reference interval evaluation of high-sensitivity troponin $\mathrm{T}$ and $\mathrm{N}$-terminal B-type natriuretic peptide in Vietnam and the US: The North South East West Trial. Clinical chemistry 2014;6o(5):758-64.

15. Gore MO, Seliger SL, Defilippi CR, et al. Age- and sex-dependent upper reference limits for the high-sensitivity cardiac troponin T assay. Journal of the American College of Cardiology 2014;63(14):1441-8.

16. Zeller T, Ojeda F, Brunner FJ, et al. High-sensitivity cardiac troponin I in the general population--defining reference populations for the determination of the $99^{\text {th }}$ percentile in the Gutenberg Health Study. Clinical chemistry and laboratory medicine : CCLM / FESCC 2015;53(5):699-706.

17. Franzini M, Lorenzoni V, Masotti S, et al. The calculation of the cardiac troponin $\mathrm{T} 99^{\text {th }}$ percentile of the reference population is affected by age, gender, and population selection: A multicenter study in Italy. Clinica chimica acta; international journal of clinical chemistry 2015;438:376-81.

18. Koerbin G, Tate J, Potter JM, et al. Characterisation of a highly sensitive troponin I assay and its application to a cardio-healthy population. Clinical chemistry and laboratory medicine: CCLM / FESCC 2012;50(5):871-8.

19. Schram MT, Sep SJ, van der Kallen CJ, et al. The Maastricht Study: an extensive phenotyping study on determinants of type 2 diabetes, its complications and its comorbidities. European journal of epidemiology 2014;29(6):439-51.

20. World Medical A. World Medical Association Declaration of Helsinki: ethical principles for medical research involving human subjects. JAMA : the journal of the American Medical Association 2013;310(20):2191-4.

21. Reed AH, Henry RJ, Mason WB. Influence of statistical method used on the resulting estimate of normal range. Clinical chemistry 1971;17(4):275-84.

22. Horn PS, Feng L, Li Y, et al. Effect of outliers and nonhealthy individuals on reference interval estimation. Clinical chemistry 2001;47(12):2137-45.

23. Wildi K, Rubini Gimenez M, Twerenbold R, et al. Misdiagnosis of Myocardial Infarction Related to Limitations of the Current Regulatory Approach to Define Clinical Decision Values for Cardiac Troponin. Circulation 2015.

24. Apple FS, Ler R, Murakami MM. Determination of 19 cardiac troponin I and T assay $99^{\text {th }}$ percentile values from a common presumably healthy population. Clinical chemistry 2012;58(11):1574-81.

25. Shah AS, Griffiths M, Lee KK, et al. High sensitivity cardiac troponin and the underdiagnosis of myocardial infarction in women: prospective cohort study. Bmj 2015;350:g7873. 


\section{SUPPLEMENTAL DATA}

\section{> THE MAASTRICHT STUDY COHORT AND DEFINING A HEALTHY REFERENCE COHORT}

The Maastricht Study focuses on the etiology, pathophysiology, complications and comorbidities of type 2 diabetes mellitus (T2DM) and is characterized by an extensive phenotyping approach. Eligible for participation were all individuals aged between 40 and 75 years and living in the southern part of the Netherlands. Participants were recruited through mass media campaigns and from the municipal registries and the regional Diabetes Patient Registry via mailings. Recruitment was stratified according to known T2DM status for reasons of efficiency.

The present report includes cross-sectional data from the first 3451 participants, who completed the baseline survey between November 2010 and September 2013. The examinations of each participant were performed within a time window of three months.

According the standardized approach of Sandoval et al. the concept "healthy" was defined. ${ }^{1}$ Key concept of this recommendation is that the screening procedure should not only consist of the screening for a clinical history for known cardiovascular disease, but that also an assessment with surrogate biomarkers for diabetes mellitus (DM), myocardial dysfunction and renal dysfunction is required. ${ }^{1}$ Accordingly, the healthy sub cohort was defined by excluding a) individuals with DM, b) individuals with a clinical history of cardiovascular disease, c) individuals with prior cardiac ischemia on electrocardiography (ECG), d) individuals with serum $\mathrm{N}$-terminal probrain natriuretic peptide (NT-proBNP) $>125 \mathrm{ng} / \mathrm{L}$ and e) individuals with an estimated Glomerular Filtration Rate (eGFR) $<60 \mathrm{~mL} /$ minute $/ 1.73 \mathrm{~m}^{2}$, calculated according to Chronic Kidney Disease Epidemiology Collaboration equation based on cystatin $\mathrm{C}$ in combination with creatinine. ${ }^{2}$ An 75 g oral glucose tolerance test was performed in all subjects to classify glucose metabolism status as normal glucose tolerance (NGT), impaired fasting glucose (IFG), impaired glucose tolerance (IGT) and T2DM according to the World Health Organization 2006 criteria. ${ }^{3}$ Clinical history for known cardiovascular disease was assessed using the Rose questionnaire. ${ }^{4}$ In addition, a resting 12-lead ECG was performed using the Mac 5500 ECG system (GE Medical Systems, Milwaukee, Wisconsin, USA). Subjects with evidence of prior cardiac ischemia on the ECG, defined according to Minnesota code classification system (Minnesota code $1.1-1.3,4.1-4.3,5.1-5.3$ or 7.1), were excluded. ${ }^{5}$ 


\begin{tabular}{|c|c|c|c|}
\hline & $\begin{array}{l}\text { Entire study } \\
\text { population } \\
(n=1540), n(\%)\end{array}$ & $\begin{array}{l}\text { Stratified } \\
\text { by men } \\
(n=733), n(\%)\end{array}$ & $\begin{array}{l}\text { Stratified } \\
\text { by women } \\
(n=807), n(\%)\end{array}$ \\
\hline \multicolumn{4}{|l|}{ hs-cTnT } \\
\hline$=\operatorname{LoB}(3 \mathrm{ng} / \mathrm{L})$ & $1256(81.6 \%)$ & $697(95.1 \%)$ & $559(69.3 \%)$ \\
\hline$\geq \operatorname{LoD}(5 \mathrm{ng} / \mathrm{L})$ & $668(43.4 \%)$ & $480(65.5 \%)$ & $188(23.3 \%)$ \\
\hline \multicolumn{4}{|l|}{ hs-cTnl } \\
\hline$=\operatorname{LoB}(0.7 \mathrm{ng} / \mathrm{L})$ & I447 (94.0\%) & $713(97.8 \%)$ & $730(90.5 \%)$ \\
\hline$\geq \operatorname{LOB}(1.3 \mathrm{ng} / \mathrm{L})$ & $1070(69.5 \%)$ & $625(85.3 \%)$ & $445(55.1 \%)$ \\
\hline$\geq \operatorname{LoD}(\mathrm{I} . \mathrm{lng} / \mathrm{L})$ & $1218(79.1 \%)$ & $672(91.7 \%)$ & $546(67.7 \%)$ \\
\hline$=\mathrm{LoD}(1.9 \mathrm{ng} / \mathrm{L})$ & $664(43.1 \%)$ & $455(62.1 \%)$ & $209(25.9 \%)$ \\
\hline \multicolumn{4}{|l|}{ combined hs-cTn } \\
\hline \multicolumn{4}{|l|}{$\geq \mathrm{LOB}$} \\
\hline hs-cTnT 3 ng/L, hs-cTnl 0.7 ng/L & $1216(79.0 \%)$ & $686(93.6 \%)$ & $530(65.7 \%)$ \\
\hline hs-cTnT 3 ng/L, hs-cTnl I.3 ng/L & $978(63.5 \%)$ & $607(82.1 \%)$ & $371(46.0 \%)$ \\
\hline \multicolumn{4}{|l|}{$\geq \operatorname{LOD}$} \\
\hline hs-cTnT 5 ng/L, hs-cTnl I.I ng/L & $636(41.3 \%)$ & $467(63.7 \%)$ & $169(20.9 \%)$ \\
\hline hs-cTnT 5 ng/L, hs-cTnl I.9 ng/L & $448(29.1 \%)$ & $351(47.9 \%)$ & $97(12.0 \%)$ \\
\hline
\end{tabular}

Supplemental Table 2.1. Percentage measurable high-sensitivity cardiac troponin levels.

\section{REFERENCES}

1. Sandoval Y, Apple FS. The global need to define normality: the $99^{\text {th }}$ percentile value of cardiac troponin. Clinical chemistry 2014;60(3):455-62.

2. Inker $\mathrm{LA}, \mathrm{Schmid} \mathrm{CH}$, Tighiouart $\mathrm{H}$, et al. Estimating glomerular filtration rate from serum creatinine and cystatin C. The New England journal of medicine 2012;367(1):20-9.

3. WHO. Definition and Diagnosis of diabetes mellitus and intermediate hyperglycemia. Secondary Definition and Diagnosis of diabetes mellitus and intermediate hyperglycemia 2006. www.whoint/diabetes/publications/Definition\%20and\%2odiagnosis\%20of\%2odiabets_new.pdf.

4. Leng GC, Fowkes FG. The Edinburgh Claudication Questionnaire: an improved version of the WHO/Rose Questionnaire for use in epidemiological surveys. Journal of clinical epidemiology 1992;45(10):1101-9.

5. Prineas RJ, Crow RS, Zhang ZM. The Minnesota Code Manual of Electrocardiographic Findings. 2nd ed: Springer-Verlag London, 2010. 

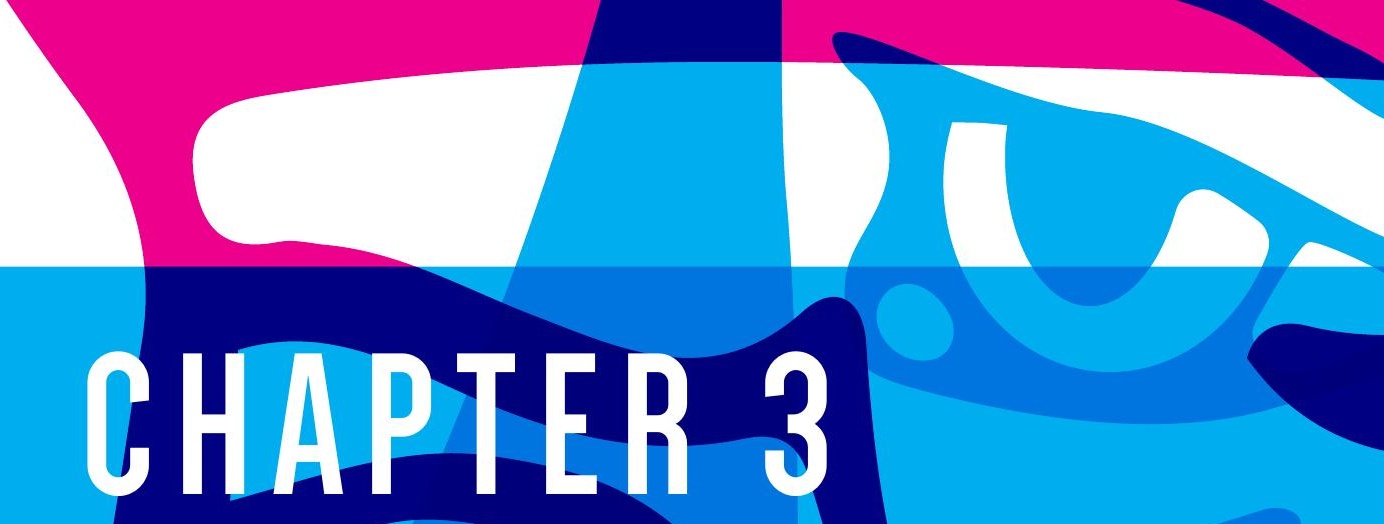



\section{CHAPTER 3}

SEX-SPECIFIC VERSUS

OVERALL CLINICAL

DECISION LIMITS FOR

CARDIAC TROPONIN

I AND TFOR THE

DIAGNOSIS OF ACUTE

MYOCARDIAL INFARCTION:

A SYSTEMATIC REVIEW

Dorien M Kimenai, Emma BNJJanssen, Kai M Eggers, Bertil Lindahl, Hester M den Ruijter, Otto Bekers, Yolande Appelman, Steven JR Meex

Clinical Chemistry 2018; 64 (7): 1034-1043 


\section{ABSTRACT}

\section{$>$ BACKGROUND}

The overall clinical decision limits of high-sensitivity cardiac troponin I (hscTnI; $26 \mathrm{ng} / \mathrm{L}$ ) and T (hs-cTnT; $14 \mathrm{ng} / \mathrm{L}$ ) may contribute to underdiagnosis of acute myocardial infarction in women. We performed a systematic review to investigate sex-specific and overall $99^{\text {th }}$ percentiles of hs-cTnI and hs-cTnT derived from healthy reference populations.

\section{$>$ CONTENT}

We searched in PubMed and EMBASE for original studies, and by screening reference lists. Reference populations designed to establish $99^{\text {th }}$ percentiles of hscTnI (Abbott) and/or hs-cTnT (Roche), published between January 2009 and October 2017 , were included. Sex-specific and overall $99^{\text {th }}$ percentile values of hs-cTnI and hs-cTnT were compared with overall clinical decision ranges (hs-cTnI, 23 - $30 \mathrm{ng} / \mathrm{L}$; hs-cTnT, $13-25 \mathrm{ng} / \mathrm{L}$ ).

Twenty-eight studies were included in the systematic review. Of 16 hs-cTnI and 18 hs-cTnT studies, 14 (87.5\%) and 11 (61.1\%) studies reported lower female-specific hscTn cut-offs than overall clinical decision ranges, respectively. Conversely, malespecific thresholds of both hs-cTnI and hs-cTnT were in line with currently used overall thresholds, particularly hs-cTnT ( $90 \%$ concordance).

The variation of estimated overall $99^{\text {th }}$ percentiles was much higher for hs-cTnI than hs-cTnT (29.4\% versus $80.0 \%$ of hs-cTnI and hs-cTnT studies reported values within the current overall clinical decision range, respectively).

\section{$>$ SUMMARY}

Our data show substantially lower female-specific upper reference limits of hscTnI and hs-cTnT than overall clinical decision limits of $26 \mathrm{ng} / \mathrm{L}$ and $14 \mathrm{ng} / \mathrm{L}$, respectively. The statistical approach strongly affects the hs-cTnI threshold. Downward adjustment of hs-cTn thresholds in women may be warranted to reduce underdiagnosis of acute myocardial infarction in women. 


\section{INTRODUCTION}

Women have lower 1-year survival rates after an acute myocardial infarction (AMI) than men. ${ }^{1,2}$ Twenty-three percent of women versus $18 \%$ of men will die within 1 year after a first AMI. ${ }^{2}$ Atypical symptomatology in women with AMI is a possible cause of their undertreatment, but underdiagnosis also may play a crucial role in the existing gap between women and men. ${ }^{3}$ Indeed, in a population-based cohort study investigating the incidence of (un)recognized AMIs in women and men, the proportion of incident AMIs remaining clinically unrecognized was higher for women than men (4\% for women versus $33 \%$ for men). ${ }^{4}$ However, the incidence of missed AMIs in women from application of the common diagnostic cardiac troponin algorithm is not well established, as prospective trials on this matter are lacking. The urgent need for conducting research to understand these disparities between women and men has recently been recognized. ${ }^{3}$

An algorithm using sex-specific cutoff concentrations for cardiac troponins is recommended, but not required, in the third universal definition of myocardial infarction. ${ }^{5}$ The European Society of Cardiology guidelines require a defined 'rise and/or fall' of high-sensitivity cardiac troponin I (hs-cTnI) or T (hs-cTnT) between serial measurements with at least 1 value above the $99^{\text {th }}$ percentile upper reference limits (URLs) of hs-cTn from a healthy reference population; however, the European Society of Cardiology guidelines do not recommend a sex-specific algorithm. ${ }^{6}$

The $99^{\text {th }}$ percentile URL of cardiac troponin from a reference population for diagnosis of AMI is proposed by the Joint European Society of Cardiology/ American College of Cardiology Committee for the Redefinition of Myocardial Infarction, driven by the demonstration that any amount of detectable troponin release is associated with an increased risk of new adverse cardiac events. ${ }^{7,8}$ Defining adequate clinical decision limits of hs-cTnI and hs-cTnT was challenging owing to the lack of a biomarker-independent gold standard for diagnosis of AMI. As a result, common clinical thresholds are based on a statistical approach rather than a biological approach (which would have been preferable). Why the $99^{\text {th }}$ percentile URL was chosen rather than the more common 97.5th percentile URL was probably a result of the much lower sensitivity (and precision) of the assays available around the millennium shift when the first Universal Definition document was created.

The 2 clinical troponin assays that meet the analytical properties of a highsensitivity assay are the Abbott ARCHITECT STAT hs-cTnI assay and the Roche hs-cTnT assay with recommended overall clinical decision limits of $26 \mathrm{ng} / \mathrm{L}$ and $14 \mathrm{ng} / \mathrm{L}$, respectively (package insert). ${ }^{9}$ 
Two concerns have been raised that may hamper the diagnosis of AMI in women. First, the clinical decision limits of hs-cTnI and hs-cTnT are overall thresholds and, therefore, make no distinction between women and men. The introduction of high-sensitivity troponin assays has led to detection of troponin concentrations in a lower range and revealed the presence of female/male differences when assessing the $99^{\text {th }}$ percentile values of hs-cTn. ${ }^{10,11}$ These data imply that the overall clinical decision limits of hs-cTn are too high for women, which may contribute to underdiagnosis of AMI. ${ }^{12}$ The second concern is that the overall clinical decision limits of hs-cTnI and hs-cTnT might not be biologically equivalent, as they are not derived from a single reference population. ${ }^{12,13}$ Wildi et al. showed that 1 out of 5 diagnoses of AMI is inconsistent with the overall clinical decision limits of hs-cTnI and hs-cTnT. Their data suggested that the overall cutoff of $26 \mathrm{ng} / \mathrm{L}$ for hs-cTnI should be lowered to $\mathrm{g}$ n/L to become biologically equivalent to the overall hs-cTnT threshold of $14 \mathrm{ng} / \mathrm{L} .{ }^{13}$ Given the lower circulating troponin concentrations in women than men, the effect of a higher diagnostic threshold for the hs-cTnI assay is possibly more problematic for women than men.

Several studies have studied healthy reference populations to establish sex-specific and overall $99^{\text {th }}$ percentile URL of hs-cTnI and/or hs-cTnT. Evaluating these sexspecific and overall cutoffs could a) provide direction on whether sex-specific clinical decision limits of hs-cTn should be considered for further investigation in randomized controlled trials, and b) assess whether the currently used overall clinical decision limits of $26 \mathrm{ng} / \mathrm{L}$ for hs-cTnI and/or $14 \mathrm{ng} / \mathrm{L}$ for hs-cTnT need critical reinvestigation. We performed a systematic review to investigate sexspecific and overall $99^{\text {th }}$ percentile URL of hs-cTnI and hs-cTnT derived from healthy reference populations.

\section{MATERIAL AND METHODS}

\section{> SEARCH STRATEGY AND ELIGIBILITY CRITERIA}

The study was conducted in according to the principles of the Preferred Reporting Items for Systematic Reviews and Meta-Analyses (PRISMA) guidelines. ${ }^{14} \mathrm{We}$ searched in PubMed and EMBASE for original studies published between January 2009 and October 2017 (see Supplemental Data). Additionally, we screened the reference lists of relevant articles.

Reference cohorts designed to assess sex-specific and/or overall $99^{\text {th }}$ percentile URL of hs-cTnI and/or hs-cTnT from healthy reference individuals $\geq 18$ years were included. Only studies written in English were included. Exclusion criteria were reference populations with a sample size $<300^{15}$, and conventional troponin assays i.e. assays other than Abbott ARCHITECT STAT hs-cTnI assay or Roche hs-cTnT assay. 


\section{STUDY SELECTION AND DATA EXTRACTION}

Studies were selected by 2 independent reviewers (DMK and EBNJJ). Initial screening of all identified records was performed on title and abstract. Of potentially eligible studies, full texts were obtained and assessed for inclusion. In case of duplicate studies or overlapping references populations, the most recently published article was selected.

Data were extracted by 2 independent reviewers (DMKand EBNJJ). All disagreements were resolved in the presence of a third reviewer (SJRM). Using a standardized form, the following information was collected: author, year of publication, troponin assay, population and setting, sample size, statistical approach, and $99^{\text {th }}$ percentile values of hs-cTn (with their 90\% CI or 95\% CI). Previously, Sandoval et al. recommended a set of criteria for defining a reference population. ${ }^{16}$ According to these criteria, we collected sex-specific and overall $99^{\text {th }}$ percentile URL from the most stringently screened selection of a reference population. ${ }^{16}$ In cases when the $99^{\text {th }}$ percentile values of hs-cTn from the most stringently screened reference cohort were not reported, this additional information was requested from the study authors by e-mail.

\section{$>$ DATA SYNTHESIS}

Owing to substantial heterogeneity in screening criteria and applied methods for determination of $99^{\text {th }}$ percentile URL, a qualitative data synthesis was performed. Female-specific, male-specific and overall $99^{\text {th }}$ percentile URL of hs-cTnI and hscTnT were depicted in graphs. Outcomes were compared with the package-insertdefined $90 \%$ or $95 \%$ CI ranges for the overall clinical decision thresholds of hs-cTnI and hs-cTnT from 23 to $30 \mathrm{ng} / \mathrm{L}$ and from 13 to $25 \mathrm{ng} / \mathrm{L}$, respectively. Such graphs demonstrate to what extent reported sex-specific $99^{\text {th }}$ percentile URLs of cardiac troponins match with currently used decision limits, and whether reconsideration of the overall clinical decision limits is warranted.

To investigate the hypothesis that reference population heterogeneity contributes to discrepancy between $99^{\text {th }}$ percentile values of hs-cTnI and hs-cTnT, we selected the studies that directly compared the $99^{\text {th }}$ percentile URL of hs-cTnI and hs-cTnT derived from a single reference population. Additionally, we investigated the differences between the $99^{\text {th }}$ percentile URLs of hs-cTnI and hs-cTnT, also stratified by sex. 


\begin{tabular}{|c|c|c|}
\hline & hs-cInl & hs-cTnT \\
\hline \multicolumn{3}{|l|}{ Study Characteristics } \\
\hline Publication & Rogers et al. $2013^{17}$ & Saenger et al. 20118 \\
\hline Country & US & US/ Europe \\
\hline Description cohort & US population & 3 US and 5 European sites \\
\hline Women/ Men (n, \%) & $765(50 \%) / 766(50 \%)$ & $265(49.7 \%) / 268(50.3 \%)$ \\
\hline Age range (years) & $21-75$ & $20-71$ \\
\hline Statistical approach & Robust & NP/Dixon \\
\hline Female-specific 99th percentile & $16[14-18]$ & 9 \\
\hline Male-specific 99th percentile & $34[29-39]$ & 16 \\
\hline Overall 99th percentile & $26[23-30]$ & $14(13-25)$ \\
\hline
\end{tabular}

Table 3.1.

Characteristics of reference populations in which package insert clinical decision limits of hs-cTnI and hs-cTnT were derived.

The ggth percentile upper reference limits are reported in $n g / L$, and are presented with their 90\% CI [ ] or $95 \% \mathrm{CI}$ (). Abbreviations: NP, nonparametric.

\section{RESULTS}

Full-text screening of 67 studies revealed that 37 studies did not meet the inclusion criteria (Supplemental Figure 3.1). The 2 original studies that were the basis for currently used decision limits of hs-cTnI and hs-cTnT were also excluded (Table 3.1).1718

Twenty-eight studies were included in the qualitative synthesis. ${ }^{10-12,19-43}$ Six studies derived $99^{\text {th }}$ percentile values for both hs-cTnI and hs-cTnT (Table 3.2, Supplemental Table 3.1 (11,12,19-22 $^{11}$ studies determined $99^{\text {th }}$ percentile values of hs-cTnI (Table 3.3, Supplemental Table 3.2 $)^{23-33}$, and 11 studies determined $99^{\text {th }}$ percentile values of hscTnT (Table 3.3, Supplemental Table 3.3). ${ }^{10,34-43}$

\section{> SEX-SPECIFIC 99TH PERCENTILE URL OF HS-CTNI AND HS-CTNT}

In almost $90 \%$ of studies (14 of 16 studies), female-specific $99^{\text {th }}$ percentile values of hs-cTnI were lower than the overall package insert-defined clinical decision CI ranges (Figure 3.1A).11,12,19-30,32,33 Regarding to the female-specific clinical decision CI range of hs-cTnI (16 ng/L; range, 14-18 ng/L; package insert), 7 (43.8\%) studies reported femalespecific female-specific $99^{\text {th }}$ percentile values below this range. Although somewhat less remarkable, 11 of $18 \mathrm{hs}$-cTnT studies $(61.1 \%)$ also reported lower female-specific $99^{\text {th }}$ percentile values than the overall clinical decision CI range (Figure 3.1B)..$^{10,12,20,22,34,35,37-41}$ 
Figure 3.1. Female-specific 9gth percentile upper reference limits of hs-cTnI (A) and hs-cTnT (B) compared with the package insert-defined 90\% or 95\% CI ranges for the overall clinical decision threshold of 23 to $30 \mathrm{ng} / \mathrm{L}$ for hs-cTnI and 13 to $25 \mathrm{ng} / \mathrm{L}$ for hs-cTnT (shaded area).
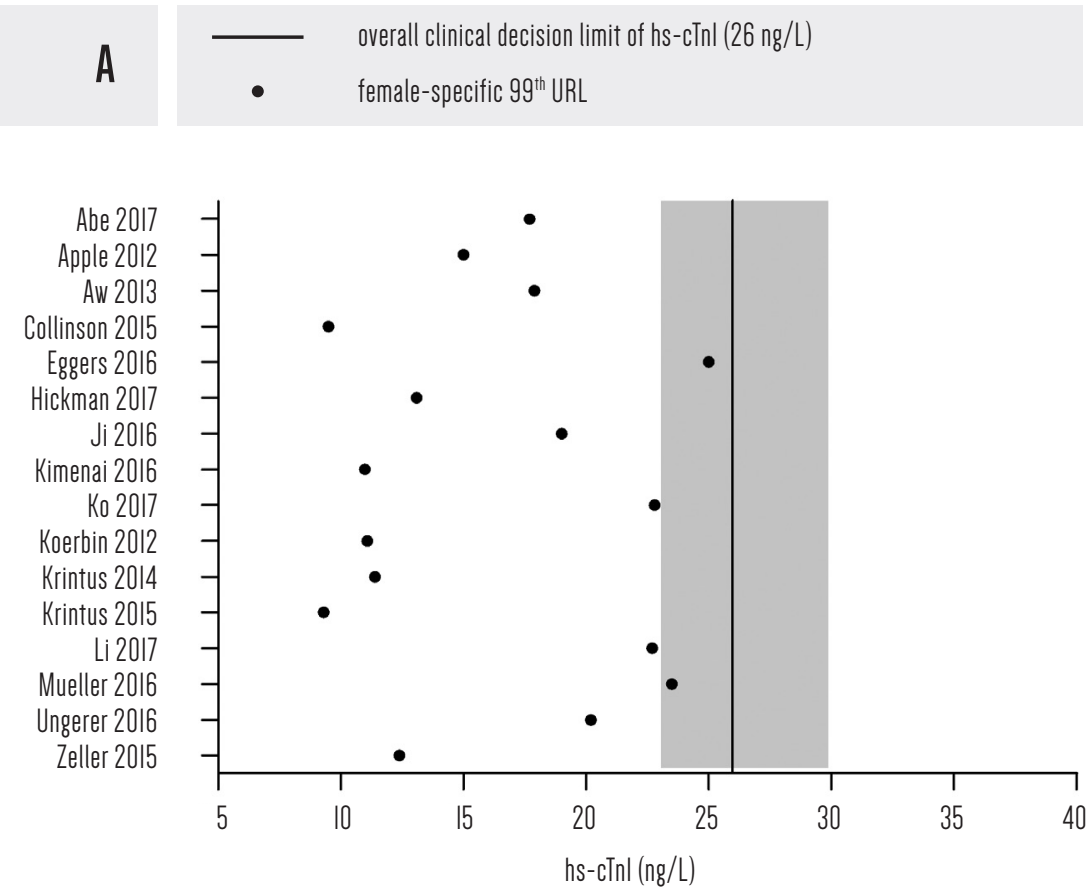

B

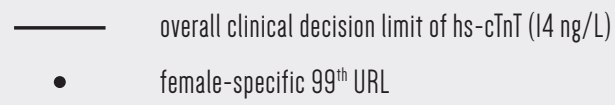
Apple 2012
Aw 2017
Collinson 2012
Franzini 2015 Gaggin 2014 US Gaggin 2014 Vietnam
Giannitsis 2010
Gore 2014 DHS
Gore 2014 ARIC
Gore 2014 CHS
Gunsolus 2017
Hammarsten 2012
Hickman 2017
Kimenai 2016
Ko 2017
Mingels 2009
Mueller 2016
Ungerer 2016

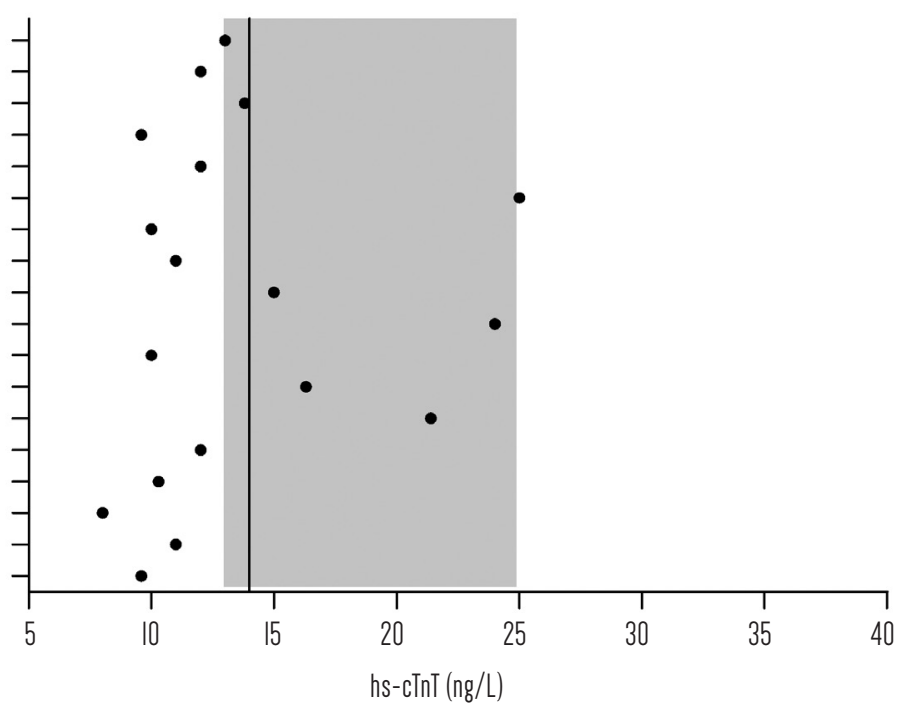




\section{A

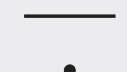 \\ overall clinical decision limit of hs-cTnl (26 ng/L) \\ overall 99th URL}

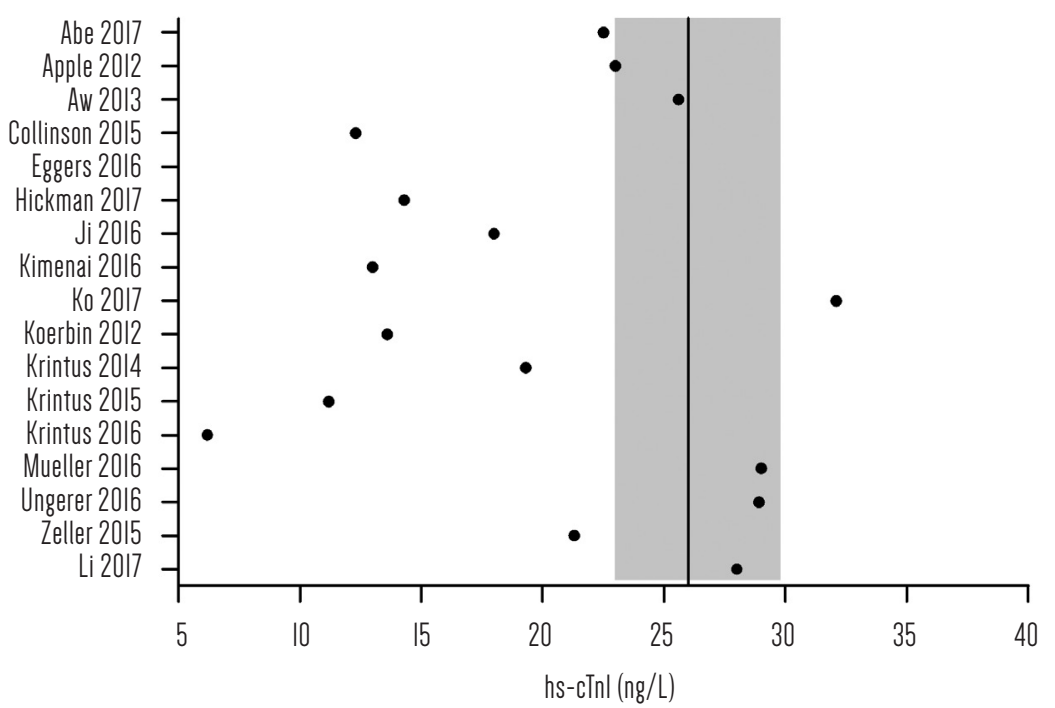

B
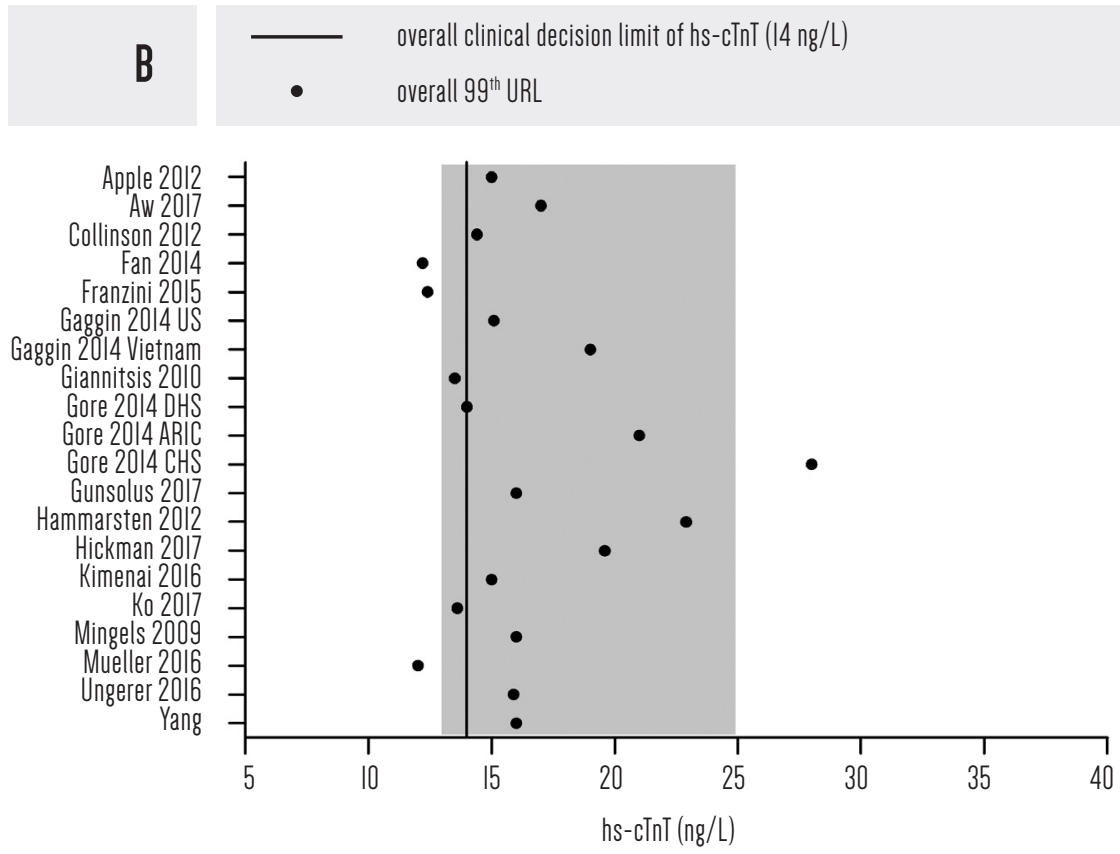

Figure 3.2.

Overall 9gth percentile upper reference limits of hs-cTnI (A) and hs-cTnT

(B) as compared with the package insert-defined 90\% or 95\% CI ranges for the overall clinical decision threshold of 23 to $30 \mathrm{ng} / \mathrm{L}$ forhs-cTnI and 13 to $25 \mathrm{ng} / \mathrm{L}$ for hs-cTnT (shaded area). 
Figure 3.3 Direct comparison of overall $99^{\text {th }}$ percentile URL

of hs-cTnI

and hs-cTnT

derived from a

single reference population. The

right column shows the

absolute numeric differences

between $99^{\text {th }}$

percentile values

of hs-cTnI and

hs-cTnT from a single reference population. Characteristics of included studies that reported sex-specific and overall $99^{\text {th }}$ percentiles of hs-cTnI and hs-cTnT $(n=6)$.

\begin{tabular}{|c|c|c|c|c|c|}
\hline Publication & Country & Cohort Description & $\begin{array}{l}\text { Female/ Male } \\
(n, \%)\end{array}$ & $\begin{array}{l}\text { Age Range } \\
\text { (years) }\end{array}$ & $\begin{array}{l}\text { Statistical } \\
\text { Approach }\end{array}$ \\
\hline Apple et al. 2012" & US & Healthy volunteers & $252(48 \%) / 273(52.0 \%)$ & $18-64$ & NP/ Dixon \\
\hline Hickman etal. $2017^{19}$ & Australia & $\begin{array}{l}\text { CHS and a } \\
\text { younger cohort of } \\
\text { population-based } \\
\text { individuals }\end{array}$ & $\begin{array}{l}\text { hs-cTnl } \\
262(54.1 \%) / 222 \text { (45.9\%) } \\
\text { hs-cTnT } \\
262(53.5 \%) / 228(46.5 \%)\end{array}$ & $48-95$ & NP/ Dixon \\
\hline Kimenai etal. 2016 ${ }^{12}$ & The Netherlands & $\begin{array}{l}\text { Healthy } \\
\text { individuals from } \\
\text { The Maastricht Study }\end{array}$ & $806(52.5 \%) / 729(47.5 \%)$ & $40-75$ & NP/ Dixon \\
\hline Ko et al. $20177^{20}$ & Korea & $\begin{array}{l}\text { Individuals seen } \\
\text { for health checks }\end{array}$ & $338(52.7 \%) / 303(47.3 \%)$ & $22-86$ & NP/ Tukey \\
\hline Mueller etal. 201621 & Austria & Blood donors & $143(36 \%) / 259(64 \%)$ & $<65$ & NP \\
\hline Ungerer et al. 2016 22 & Australia & Blood donors & 705 (35.2\%)/ I 299 (64.8\%) & Not reporte & NP \\
\hline
\end{tabular}

- - - - - o overall clinical decision limit of hs-cTnl ( $26 \mathrm{ng} / \mathrm{L}$ )

-----ー overall clinical decision limit of hs-cTnT (I4 ng/L)

g9th URL - hs-cTnl

$\Delta \quad g^{\text {th }}$ URL - hs-cTnT

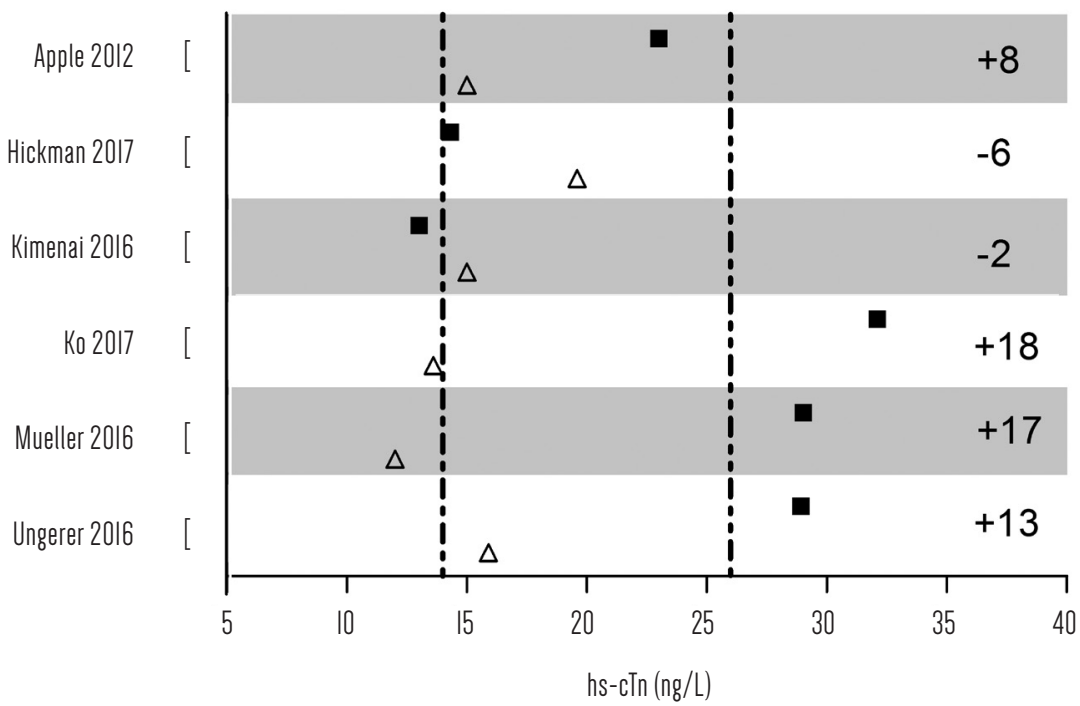

hs-cTn (ng/L) 
In men, the $99^{\text {th }}$ percentile values of both hs-cTnI and hs-cTnT are more in line with currently used overall thresholds. For hs-cTnT, almost $90 \%$ of the male-specific $99^{\text {th }}$ percentile URL match with current clinical practice ${ }^{10-12,19,20,22,34,35,38-43}$, although values of hs-cTnI showed a much wider dispersion around the recommended overall threshold (Supplemental Figure 3.2). ${ }^{11,12,19-30,32,33}$

\section{$>$ OVERALL 99TH PERCENTILE URL OF HS-CTNI AND HS-CTNT}

Several studies (17 and 20, respectively) determined the overall $99^{\text {th }}$ percentiles of hs-cTnI and hs-cTnT (Supplemental Tables $3.1-3 \cdot 3$ ) ${ }^{10-12,19-43}$ The majority of studies $(58.8 \%)$ that determined overall $99^{\text {th }}$ percentile URL for hs-cTnI reported lower $99^{\text {th }}$ percentile values than the clinically recommended overall decision range in the package insert $(29.4 \%$ within and $11.8 \%$ above the overall clinical decision range) (Figure 3.2A). In line with the sex stratified analysis, the range of overall $99^{\text {th }}$ percentiles was more dispersed for hs-cTnI than for hs-cTnT (Figure 3.2B).

\section{> DIRECT COMPARISON OF 99TH PERCENTILE URL FOR HS-CTNI AND HS-CTNT}

To investigate whether the package insert overall clinical decision limits of $26 \mathrm{ng} / \mathrm{L}$ for hs-cTnI and $14 \mathrm{ng} / \mathrm{L}$ for hs-cTnT could be discrepant owing to differences in the reference populations from which they were derived, we additionally investigated the 6 studies that derived $99^{\text {th }}$ percentiles of both hs-cTn from a single reference population..$^{11,12,19-22}$ Examining the absolute numeric differences between $99^{\text {th }}$ percentile concentrations of hs-cTnI and hs-cTnT as a measure of dispersion, these values ranged from -6 to $18 \mathrm{ng} / \mathrm{L}$ (Figure 3.3).

The dispersion seems less an issue in women than in men (absolute difference $99^{\text {th }}$ percentile hs-cTnT - hs-cTnI: women, -1 to $13 \mathrm{ng} / \mathrm{L}$; men, 4 to $30 \mathrm{ng} / \mathrm{L}$ ) (Supplemental Figure 3.3). 


\begin{tabular}{|c|c|c|c|}
\hline $\begin{array}{r}\text { Table } 3.3 \text {. } \\
\text { Characteristics of }\end{array}$ & Publication & Country & Cohort Description \\
\hline & hs-cTnl studies ( $=$ II) & & \\
\hline & Abe et al. $2018^{23}$ & Japan & $\begin{array}{l}\text { Individuals who visited the Japanese Red Cross Medical Center } \\
\text { for health checkup }\end{array}$ \\
\hline $\begin{array}{l}\text { percentiles of } \\
\text { hs-cTnI or }\end{array}$ & Aw et al. $2013^{24}$ & Singapore & Individuals participating in health screening program \\
\hline$n s-\operatorname{ctn} 1(n=22)$. & Collinson et al. 201525 & UK & $\begin{array}{l}\text { Individuals from seven representative local community } \\
\text { practices in Harrow. North London }\end{array}$ \\
\hline & Eggers et al. $2016^{26}$ & Sweden & Community inhabitants of the PIVUS study \\
\hline & Ji et al. $2016^{27}$ & South-Korea & Individuals participating in health screening program \\
\hline & Koerbin et al. $2012^{28}$ & Australia & CHS and a younger cohort of population-based individuals \\
\hline & Krintus et al. $2014^{29}$ & Poland & $\begin{array}{l}\text { Apparently healthy individuals and blood donors recruited } \\
\text { in seven European countries }\end{array}$ \\
\hline & Krintus et al. $2015^{30}$ & Poland & $\begin{array}{l}\text { Presumably healthy individuals from } 9 \text { European countries } \\
\text { (health check-ups, GHS, NOBIDA, healthy volunteers) }\end{array}$ \\
\hline & Krintus et al. $2016^{31 *}$ & Poland & $\begin{array}{l}\text { Presumably healthy individuals recruited in various } \\
\text { workplaces in Bydgoszcz }\end{array}$ \\
\hline & Li et al. $2017^{32}$ & China & Individuals seen for health checkup \\
\hline & Zeller et al. $2015^{33}$ & Germany & GHS \\
\hline & hs-cInT studies ( $\mathrm{n}=\mathrm{II})$ & & \\
\hline & Aw et al. $2017^{34}$ & Singapore & Individuals participating in a health screening program \\
\hline & Collinson et al. $2012^{35}$ & UK & $\begin{array}{l}\text { Individuals from seven representative local community practices } \\
\text { in Harrow, North London }\end{array}$ \\
\hline & Fan et al. $2014^{36 *}$ & China & Individuals seen for health check \\
\hline & Franzini et al. $2015^{37}$ & Italy & $\begin{array}{l}\text { G. Monasterio Tuscany Foundation, San Maurizio } \\
\text { Regional Hospital, MEHLP, CAMELLIA and MAREA }\end{array}$ \\
\hline & Gaggin et al. $2014^{38}$ & US/ Vietnam & NSEW Trial \\
\hline & Giannitsis et al. $2010^{39}$ & Germany & Apparently healthy volunteers and blood donors \\
\hline & Gore et al. $2014^{40}$ & US & DHS/ARIC/ CHS \\
\hline & Gunsolus et al. $2017^{41}$ & US & Subjects from the AACC Universal Sample Bank \\
\hline & Hammarsten et al. $2012^{42 *}$ & Sweden & MONICA study \\
\hline & Mingels et al. 200910 & The Netherlands & Individuals seen for health check \\
\hline & Yang et al. $2016^{43 *}$ & China & Individuals from Northern China, covering cities and villages. \\
\hline
\end{tabular}




\begin{tabular}{lll} 
Female/ Male (n. \%) & Age Range (years) & Statistical Approach \\
\hline $385(55.2 \%) / 313(44.8 \%)$ & $23-86$ & $\begin{array}{l}\text { Smoothed empirical } \\
\text { likelihood/ Dixon }\end{array}$ \\
$523(46.7 \%) / 597(53.3 \%)$ & $35-65$ & NP \\
$195(53.3 \%) / 171(46.7 \%)$ & $>45$ & NP \\
$255(48.9 \%) / 266(51.1 \%)$ & 70 & NP/ Dixon \\
$428(50.1 \%) / 426(49.9 \%)$ & $18-90$ & NP/ Dixon \\
$276(55.5 \%) / 221(44.5 \%)$ & $20-84$ & not reported \\
$776(43.9 \%) / 993(56.1 \%)$ & $18-9 \mid$ & NP/ Dixon \\
$354(55.8 \%) / 280(44.2 \%)$ & GHS 35- 74 & NP/ Dixon \\
$208(51.0 \%) / 200(49.0 \%)$ & NOBIDA = 18 & NP/ Dixon \\
$754(50.8 \%) / 73 \mid(49.2 \%)$ & $18-70$ & NP \\
\hline $316(50.4 \%) / / 292(49.6 \%)$ & $18-85$ & Hajek's method \\
\hline
\end{tabular}

\begin{tabular}{|c|c|c|c|}
\hline $543(50 \%) / 543(50 \%)$ & $40-65$ & NP & \\
\hline $170(54.1 \%) / / 144(45.9 \%)$ & $>45$ & NP & \\
\hline $305(44.9 \%) / 374(55.1 \%)$ & $18-89$ & NP & \\
\hline $368(43.0 \%) / 488(57.0 \%)$ & $20-64$ & NP/ Tukey & \\
\hline $\begin{array}{l}\text { US · } 312(55.2 \%) / 253(44.8 \%) \\
\text { Vietnam • } 292(49.3 \%) / 300(50.7 \%)\end{array}$ & $>18$ & Not reported & \\
\hline $307(49.8 \%) / 309(50.2 \%)$ & $20-71$ & Not reported & \\
\hline $\begin{array}{l}\text { DHS · } 1105(55.9 \%) / 873(44.1 \%) / \text { ARIC · } 4603(60.8 \%) / 2972(39.2 \%) \\
\text { / CHS · } 885(64.4 \%) / 489(35.6 \%)\end{array}$ & $\begin{array}{l}\text { DHS } 30-65 \text { / ARIC } 45-64 \\
\text { / CHS > } 65\end{array}$ & NP & \\
\hline $339(48.8 \%) / 355(51.2 \%)$ & $|9-9|$ & NP/ Dixon & $\begin{array}{l}{ }^{*} \text { No female- } \\
\text { specific and/or }\end{array}$ \\
\hline $315(78.2 \%) / 88(21.8 \%)$ & $25-64$ & NP & $\begin{array}{l}\text { male-specific } \\
\text { 99th percentile } \\
\text { upper reference }\end{array}$ \\
\hline $215(44.9 \%) / 264(55.1 \%)$ & $\geq 18$ & NP & $\begin{array}{l}\text { limits of } \\
\text { hs-cTn reported. } \\
\text { Abbreviations: }\end{array}$ \\
\hline $661(53.0 \%) / 585(47.0 \%)$ & $18-<70$ & NP/ Dixon & $\begin{array}{l}\text { NP, non- } \\
\text { parametric. }\end{array}$ \\
\hline
\end{tabular}




\section{DISCUSSION}

The main finding of our systematic review is that female-specific $99^{\text {th }}$ percentile URL of hs-cTn are lower than the package insert values currently recommended in clinical practice, especially for hs-cTnI. In contrast with female-specific $99^{\text {th }}$ percentile values, the male-specific $99^{\text {th }}$ percentile values are more in line with those in current clinical practice. Furthermore, we found a much wider range of reported overall $99^{\text {th }}$ percentile URL for hs-cTnI than for hs-cTnT.

Although sex differences in basal concentrations of circulating troponin were first recognized in $2009^{10}$, the question of whether this finding requires a change in clinical practice is an ongoing topic of debate. Lower troponin concentrations in women are probably the result of several factors. ${ }^{44}$ The difference in left ventricular mass between sexes is most likely the main contributor to the differences in baseline troponin concentration between sexes. ${ }^{45-47}$ Better understanding of the pathogenesis of coronaryarterydiseasealsorevealed differencesbetweenwomenand men. ${ }^{48}$ The sex-hormone estrogen seems to have a protective role in the development of coronary artery disease for women by the attenuation of several processes of cardiac remodeling, reflected in lower troponin concentrations for women than men. ${ }^{49-51}$

As our findings strongly suggest that the female-specific troponin $99^{\text {th }}$ percentile URL of hs-cTnI is lower than the overall clinical decision limit of $26 \mathrm{ng} / \mathrm{L}$, it is urgently recommended to further investigate the clinical relevance of this observation. The choice to implement sex-specific analysis of hs-cTn should be carefully weighed, and potential clinical benefit should be evaluated in relation to the already advanced diagnostic algorithms of AMI using cardiac troponins. According to the guidelines, the $99^{\text {th }}$ percentile URL of hs-cTn should be above the $10 \% \mathrm{CV}$ thresholds (hs-cTnI, $5 \mathrm{ng} / \mathrm{L}$; hs-cTnT, $13 \mathrm{ng} / \mathrm{L}$ ), to ensure that detected differences are within analytical error margins to avoid misclassification of AMI diagnosis..$^{52}$ For hs-cTnI, all reported CIs of female-specific $99^{\text {th }}$ percentile URL of hs-cTnI were above the $10 \% \mathrm{CV}$ threshold of $5 \mathrm{ng} / \mathrm{L}$, making downward adjustment of hs-cTnI threshold for diagnosis of AMI in women seem feasible. Examining the analytical properties of hs-cTnT assay, the CIs for the femalespecific $99^{\text {th }}$ percentile URL of hs-cTnT were predominantly below the $10 \% \mathrm{CV}$ threshold of $13 \mathrm{ng} / \mathrm{L}$.

A plausible argument against sex-specific algorithms is that women suspected of AMI are, on average, 10 years older than men. Therefore, the age effect on troponin concentrations might neutralize the sex difference..$^{53}$ Indeed, Eggers et al. determined $99^{\text {th }}$ percentile URL from a reference cohort of 70 years of age, and reported a substantially higher female-specific $99^{\text {th }}$ percentile of hs-cTnI as compared with the other studies. ${ }^{26}$ In addition, age-adjusted 30 -day mortality after 
AMI is similar between women and men, suggesting that for the consideration of sex-specific thresholds of hs-cTn, age is an important confounding factor that should be considered. ${ }^{2}$

On the other hand, lowering the clinical decision limit of hs-cTnI might detect the subgroup of women who are at high risk and currently missed. ${ }^{54}$ This hypothesis is reinforced by Shah et al., who showed that sex-specific thresholds for hs-cTnI resulted in a similar prevalence of AMI diagnoses between women and men. ${ }^{55}$ Whether reclassification by sex-specific thresholds leads to better treatment - and accordingly, improved prognosis after AMI in women - needs to be confirmed by future prospective randomized trials.

We observed a large dispersion in the range of reported overall $99^{\text {th }}$ percentile values of hs-cTnI, which might have led to nonbiologically equivalent clinical decision limits of hs-cTnI and hs-cTnT. To investigate the influence of reference cohort variation on estimated $99^{\text {th }}$ URL, we compared the $99^{\text {th }}$ percentile URL of hs-cTnI and hs-cTnT derived from a single reference population. Even when derived from a single cohort, absolute differences up to $18 \mathrm{ng} / \mathrm{L}$ were observed between troponin I and $\mathrm{T}$ thresholds. Hence, this indicates that the divergence between the clinical decision limits of hs-cTnI and hs-cTnT is not simply a result of heterogeneity across reference populations. We believe that the dispersion seems rather to be a result of the higher proportion of outliers for hs-cTnI as previously observed..$^{12} \mathrm{~A}$ small proportion of hs-cTn values in the extreme right tail of the distribution determines the $99^{\text {th }}$ percentiles URL for hs-cTnI and hscTnT. The hs-cTnI distribution is more profoundly affected by extreme outliers than hs-cTnT distribution. ${ }^{12}$ Thus, the $99^{\text {th }}$ percentile URL is highly sensitive to outliers, and a sufficiently large sample size is an absolute requirement. Most studies that directly compared thresholds of hs-cTnI and hs-cTnT included only a limited number of reference individuals. Hence, the play of chance may have resulted in the discrepant $99^{\text {th }}$ percentiles for hs-cTnI and hs-cTnT.

Therefore, handling of outliers seems a critical factor for the determination of an appropriate clinical cutoff for hs-cTnI. For hs-cTnI, much wider CIs around the $99^{\text {th }}$ percentile values have been reported than for hs-cTnT. As most of the CIs of hs-cTn are derived from bootstraps, the wider intervals for hs-cTnI could be explained by the higher number of hs-cTnI outliers, rather than biological variation. In addition to the outlier detection method, the heterogeneity in the applied statistical approaches for calculating the $99^{\text {th }}$ percentile values also may have resulted in the large dispersion of values for hs-cTnI. Indeed, 2 studies recently showed that the applied statistical approach highly influences the $99^{\text {th }}$ percentile URL of hs-cTn..$^{19,26}$ They both conclude that the non-parametric approach in combination with a conservative outlier detection method (e.g., Dixon) is the preferred method for determination of the $99^{\text {th }}$ percentile URL for hs-cTn. ${ }^{19,26}$ 
The following limitations of our study require attention: 1) Only the hs-cTnI assay from Abbott was investigated, precluding the extrapolation of our findings to other troponin I assays. 2) Although Sandoval et al. recommended criteria for defining a healthy reference population, we believe that it was not appropriate to translate this into "hard" quality criteria owing to the wide variety of population differences globally (e.g. European versus US). Therefore, we reported the $99^{\text {th }}$ percentile URL derived from the most stringently screened selection of a reference population according to the Sandoval recommendations. 3) The statistical approach used for estimation of the $99^{\text {th }}$ percentile URL was highly variable across studies, preventing the calculation of numerical $99^{\text {th }}$ percentiles. We chose to use a less quantitative approach and evaluated whether the reported $99^{\text {th }}$ percentile URL matched with common practice. The clinical decision ranges of hs-cTnI and hs-cTnT derived from the package inserts were pragmatically defined as "common practice", as a gold standard diagnostic threshold for both hs-cTn assays is not established. 4) Furthermore, the focus in this study was on the reported $99^{\text {th }}$ percentile URL for hs-cTnI and hs-cTnT from healthy reference populations. The relation of downward adjustment of the diagnostic cutoff of hs-cTnI in relation to clinical outcome should be the subject of future research.

In conclusion, this systematic review shows that the female-specific $99^{\text {th }}$ percentile URL of hs-cTnI is lower than the overall clinical decision limit of $26 \mathrm{ng} / \mathrm{L}$. A similar, but less profound, effect is seen for hs-cTnT. Direct comparison of $99^{\text {th }}$ percentile URL of hs-cTnI and hs-cTnT revealed that the statistical approach, rather than reference population heterogeneity, contributes to current discrepant clinical decision limits. Handling of outliers seems particularly critical for the hs-cTnI threshold. Our study results suggest that future research is needed on this topic, particularly to investigate whether downward adjustment of hs-cTn thresholds, particularly hs-cTnI, can close the diagnostic gap between women and men and improve prognosis of women. 
REFERENCES

1. Pagidipati NJ, Peterson ED. Acute coronary syndromes in women and men. Nat Rev Cardiol 2016;13:471-80.

2. Benjamin EJ, Blaha MJ, Chiuve SE, Cushman M, Das SR, Deo R, et al. Heart Disease and Stroke Statistics-2017 Update: A Report From the American Heart Association. Circulation 2017;135:e146-e6o3.

3. Madonis SM, Skelding KA, Roberts M. Management of acute coronary syndromes: special considerations in women. Heart 2017;103:1638-46.

4. de Torbal A, Boersma E, Kors JA, van Herpen G, Deckers JW, van der Kuip DA, et al. Incidence of recognized and unrecognized myocardial infarction in men and women aged 55 and older: the Rotterdam Study. Eur Heart J 2006;27:729-36.

5. Thygesen K, Alpert JS, Jaffe AS, Simoons ML, Chaitman BR, White HD, et al. Third universal definition of myocardial infarction. J Am Coll Cardiol 2012;60:1581-98.

6. Roffi M, Patrono C, Collet JP, Mueller C, Valgimigli M, Andreotti F, et al. 2015 ESC Guidelines for the management of acute coronary syndromes in patients presenting without persistent ST-segment elevation: Task Force for the Management of Acute Coronary Syndromes in Patients Presenting without Persistent ST-Segment Elevation of the European Society of Cardiology (ESC). Eur Heart J 2016;37:267-315.

7. Alpert JS, Thygesen K, Antman E, Bassand JP. Myocardial infarction redefined-a consensus document of the Joint European Society of Cardiology|American College of Cardiology Committee for the redefinition of myocardial infarction. J Am Coll Cardiol 2000;36:959-69.

8. Panteghini M, Pagani F, Yeo KT, Apple FS, Christenson RH, Dati F, et al. Evaluation of imprecision for cardiac troponin assays at low-range concentrations. Clin Chem 2004;50:327-32.

9. Apple FS, Collinson PO, Biomarkers ITFoCAoC. Analytical characteristics of highsensitivity cardiac troponin assays. Clin Chem 2012;58:54-61.

10. Mingels A, Jacobs L, Michielsen E, Swaanenburg J, Wodzig W, van Dieijen-Visser M. Reference population and marathon runner sera assessed by highly sensitive cardiac troponin $\mathrm{T}$ and commercial cardiac troponin $\mathrm{T}$ and I assays. Clin Chem 2009;55:101-8.

11. Apple FS, Ler R, Murakami MM. Determination of 19 cardiac troponin I and T assay $99^{\text {th }}$ percentile values from a common presumably healthy population. Clin Chem 2012;58:1574-81.

12. Kimenai DM, Henry RM, van der Kallen CJ, Dagnelie PC, Schram MT, Stehouwer CD, et al. Direct comparison of clinical decision limits for cardiac troponin $\mathrm{T}$ and I. Heart 2016;102:610-6. 
13. Wildi K, Gimenez MR, TwerenboldR, Reichlin T, Jaeger C, Heinzelmann A, et al. Misdiagnosis of Myocardial Infarction Related to Limitations of the Current Regulatory Approach to Define Clinical Decision Values for Cardiac Troponin. Circulation 2015;131:2032-40.

14. Moher D, Liberati A, Tetzlaff J, Altman DG, Group P. Preferred reporting items for systematic reviews and meta-analyses: The PRISMA statement. Br Med J 2009;339:b2535.

15. Thygesen K, Mair J, Katus H, Plebani M, Venge P, Collinson P, et al. Recommendations for the use of cardiac troponin measurement in acute cardiac care. Eur Heart J 2010;31:2197-204.

16. Sandoval Y, Apple FS. The global need to define normality: The $99^{\text {th }}$ percentile value of cardiac troponin. Clin Chem 2014;60:455-62.

17. Rogers J, Du S, Yen J, Wiesner D, Workman R, Badciong J. Determination of a $99^{\text {th }}$ percentile for the architect stat high sensitive troponin-I immunoassay using a robust statistical method. Clin Chem 2013;59:A225.

18. Saenger AK, Beyrau R, Braun S, Cooray R, Dolci A, Freidank H, et al. Multicenter analytical evaluation of a high-sensitivity troponin T assay. Clin Chim Acta 2011;412:748-54.

19. Hickman PE, Koerbin G, Potter JM, Abhayaratna WP. Statistical considerations for determining high-sensitivity cardiac troponin reference intervals. Clin Biochem 2017;50:502-5.

20. Ko DH, Jeong TD, Cho EJ, Lim J, Ji M, Lee K, et al. The $99^{\text {th }}$ percentile values of six cardiac troponin assays established for a reference population using strict selection criteria. Clin Chim Acta 2017;464:1-5.

21. Mueller T, Egger M, Leitner I, Gabriel C, Haltmayer M, Dieplinger B. Reference values of galectin-3 and cardiac troponins derived from a single cohort of healthy blood donors. Clin Chim Acta 2016;456:19-23.

22. Ungerer JP, Tate JR, Pretorius CJ. Discordance with 3 Cardiac Troponin I and T assays: Implications for the $99^{\text {th }}$ Percentile Cutoff. Clin Chem 2016;62:1106-14.

23. Abe N, Tomita K, Teshima M, Kuwabara M, Sugawa S, Hinata N, et al. Distribution of cardiac troponin I in the Japanese general population and factors influencing its concentrations. J Clin Lab Anal 2018;32(3).

24. Aw TC, Phua SK, Tan SP. Measurement of cardiac troponin I in serum with a new high-sensitivity assay in a large multi-ethnic Asian cohort and the impact of gender. Clin Chim Acta 2013;422:26-8.

25. Collinson PO, Gaze D, Goodacre S. The clinical and diagnostic performance characteristics of the high sensitivity Abbott cardiac troponin I assay. Clin Biochem 2015;48:275-81.

26. Eggers KM, Apple FS, Lind L, Lindahl B. The applied statistical approach highly influences the $99^{\text {th }}$ percentile of cardiac troponin I. Clin Biochem 2016;49:1109-12.

27. Ji M, Moon HW, Hur M, Yun YM. Determination of high-sensitivity cardiac troponin I $99^{\text {th }}$ percentile upper reference limits in a healthy Korean population. Clin Biochem 2016;49:756-61.

28. Koerbin G, Tate J, Potter JM, Cavanaugh J, Glasgow N, Hickman PE. Characterisation of a highly sensitive troponin I assay and its application to a cardio-healthy population. Clin Chem Lab Med 2012;50:871-8.

29. Krintus M, Kozinski M, Boudry P, Capell NE, Koller U, Lackner K, et al. European multicenter analytical evaluation of the Abbott ARCHITECT STAT high sensitive troponin I immunoassay. Clin Chem Lab Med 2014;52:1657-65.

30. Krintus M, Kozinski M, Boudry P, Lackner K, Lefevre G, Lennartz L, et al. Defining normality in a European multinational cohort: Critical factors influencing the $99^{\text {th }}$ percentile upper reference limit for high sensitivity cardiac troponin I. Int J Cardiol 2015;187:256-63. 
31. Krintus M, Kozinski M, Fabiszak T, Kuligowska-Prusinska M, Laskowska E, Lennartz L, et al. Impact of lipid markers and high-sensitivity C-reactive protein on the value of the $99^{\text {th }}$ percentile upper reference limit for high-sensitivity cardiac troponin I. Clin Chim Acta 2016;462:193-200.

32. Li S, Zuo Y, Huang W. Establishment of a reference interval for high-sensitivity cardiac troponin I in healthy adults from the Sichuan area. Medicine (Baltimore) 2017;96:e6252.

33. Zeller T, Ojeda F, Brunner FJ, Peitsmeyer P, Munzel T, Binder H, et al. High-sensitivity cardiac troponin I in the general population--defining reference populations for the determination of the $99^{\text {th }}$ percentile in the Gutenberg Health Study. Clin Chem Lab Med 2015;53:699-706.

34. Aw $T$, Phua S, Lam $C$, Wong $M$. What are normal high-sensitivity troponin-T values in a large multi-ethnic Asian population? Blood, Heart and Circulation 2017;1:1-4.

35. Collinson PO, Heung YM, Gaze D, Boa F, Senior R, Christenson R, Apple FS. Influence of population selection on the $99^{\text {th }}$ percentile reference value for cardiac troponin assays. Clin Chem 2012;58:219-25.

36. Fan L, Yu P, Yu S, Gu Y, Zong M, Cai Y, Liu Z. Age-specific $99^{\text {th }}$ percentile cutoff of highsensitivity cardiac troponin $\mathrm{T}$ for early prediction of non-ST-segment elevation myocardial infarction (NSTEMI) in middle-aged patients. J Clin Lab Anal 2014;28:10-5.

37. Franzini M, Lorenzoni V, Masotti S, Prontera C, Chiappino D, Latta DD, et al. The calculation of the cardiac troponin $\mathrm{T} 99^{\text {th }}$ percentile of the reference population is affected by age, gender, and population selection: a multicenter study in Italy. Clin Chim Acta 2015;438:376-81.

38. Gaggin HK, Dang PV, Do LD, deFilippi CR, Christenson RH, Lewandrowski EL, et al. Reference interval evaluation of high-sensitivity troponin $\mathrm{T}$ and $\mathrm{N}$-terminal B-type natriuretic peptide in Vietnam and the US: The North South East West Trial. Clin Chem 2014;60:758-64.

39. Giannitsis E, Kurz K, Hallermayer K, Jarausch J, Jaffe AS, Katus HA. Analytical validation of a high-sensitivity cardiac troponin T assay. Clin Chem 2010;56:254-61.

40. Gore MO, Seliger SL, Defilippi CR, Nambi V, Christenson RH, Hashim IA, et al. Age- and sex-dependent upper reference limits for the high-sensitivity cardiac troponin $\mathrm{T}$ assay. J Am Coll Cardiol 2014;63:1441-8.

41. Gunsolus IL, Jaffe AS, Sexter A, Schulz K, Ler R, Lindgren B, et al. Sex-specific $99^{\text {th }}$ percentiles derived from the AACC Universal Sample Bank for the Roche Gen 5 cTnT assay: Comorbidities and statistical methods influence derivation of reference limits. Clin Biochem 2017;50:1073-7.

42. Hammarsten O, Fu ML, Sigurjonsdottir R, Petzold M, Said L, Landin-Wilhelmsen K, et al. Troponin $\mathrm{T}$ percentiles from a random population sample, emergency room patients and patients with myocardial infarction. Clin Chem 2012;58:628-37.

43. Yang S, Huai W, Qiao R, Cui L, Liu G, Wu J, et al. Age and Gender Tailored Cutoff Value of hscTnT Contributes to Rapidly Diagnose Acute Myocardial Infarction in Chest Pain Patients. Clin Lab 2016;62:1451-9.

44. Eggers KM, Lindahl B. Impact of Sex on Cardiac Troponin Concentrations-A Critical Appraisal. Clin Chem 2017;63:1457-64.

45. de Simone G, Devereux RB, Daniels SR, Meyer RA. Gender differences in left ventricular growth. Hypertension 1995;26:979-83.

46. de Lemos JA, Drazner MH, Omland T, Ayers CR, Khera A, Rohatgi A, et al. Association of troponin $\mathrm{T}$ detected with a highly sensitive assay and cardiac structure and mortality risk in the general population. JAMA 2010;304:2503-12. 
47. Neeland IJ, Drazner MH, Berry JD, Ayers CR, deFilippi C, Seliger SL, et al. Biomarkers of chronic cardiac injury and hemodynamic stress identify a malignant phenotype of left ventricular hypertrophy in the general population. J Am Coll Cardiol 2013;61:187-95.

48. Jaffe AS, Apple FS. High-sensitivity cardiac troponin assays: isn't it time for equality? Clin Chem 2014;60:7-9.

49. Westerman S, Wenger NK. Women and heart disease, the underrecognized burden: sex differences, biases, and unmet clinical and research challenges. Clin Sci 2016;130:551-63.

50. Donaldson C, Eder S, Baker C, Aronovitz MJ, Weiss AD, Hall-Porter M, et al. Estrogen attenuates left ventricular and cardiomyocyte hypertrophy by an estrogen receptordependent pathway that increases calcineurin degradation. Circ Res 2009;104:265-75.

51. Piro M, Della Bona R, Abbate A, Biasucci LM, Crea F. Sex-related differences in myocardial remodeling. J Am Coll Cardiol 2010;55:1057-65.

52. Sheehan P, Blennerhassett J, Vasikaran SD. Decision limit for troponin I and assay performance. Ann Clin Biochem 2002;39:231-6.

53. Twerenbold R, Boeddinghaus J, Nestelberger T, Wildi K, Rubini Gimenez M, Badertscher P, Mueller C. Clinical Use of High-Sensitivity Cardiac Troponin in Patients With Suspected Myocardial Infarction. J Am Coll Cardiol 2017;70:996-1012.

54. Cullen L, Greenslade JH, Carlton EW, Than M, Pickering JW, Ho A, et al. Sex-specific versus overall cut points for a high sensitivity troponin I assay in predicting 1-year outcomes in emergency patients presenting with chest pain. Heart 2016;102:120-6.

55. Shah AS, Griffiths M, Lee KK, McAllister DA, Hunter AL, Ferry AV, et al. High sensitivity cardiac troponin and the under-diagnosis of myocardial infarction in women: prospective cohort study. Br Med J 2015;350:g7873. 


\section{SUPPLEMENTAL DATA. LITERATURE SEARCH STRATEGIES}

\section{>I. PUBMED SEARCH (SEARCH DATE: 17-I0-2017, RESULTS, N = 1290)}

("reference values"[MeSH Terms] OR (reference[All Fields] AND value[All Fields]) OR (normal[All Fields] AND value[All Fields]) OR ( $99^{\text {th }}$ [All Fields] AND centile[All Fields]) OR (clinical [All Fields] AND decision[All Fields] AND limit[All Fields]) OR (upper[All Fields] AND reference[All Fields] AND limit[All Fields]) OR ( $99^{\text {th }}[$ All Fields] AND percentile[All Fields])) AND (hs-cTn[All Fields] OR hstnt[All Fields] OR hstni[All Fields] OR hs-cTnT[All Fields] OR hs-cTnI[All Fields] OR hsctnt[All Fields] OR hsctni[All Fields] OR ctnt[All Fields] OR ctni[All Fields] OR troponin[MeSH Terms] OR "troponin I"[MeSH Terms] OR "troponin T"[MeSH Terms] OR troponin[All Fields] OR (high[All Fields] AND sensitivity[All Fields] AND (troponin[MeSH Terms] OR troponin[All Fields]))) AND ((reference[All Fields] AND (population[MeSH Terms] OR population[All Fields] OR "population groups"[MeSH Terms])) OR (normal[All Fields] AND (population[MeSH Terms] OR population[All Fields] OR "population groups"[MeSH Terms])) OR (healthy[All Fields] AND (population[MeSH Terms] OR population[All Fields] OR "population group"[All Fields])) OR (healthy[All Fields] AND individual[All Fields]) OR ((health[MeSH Terms] OR health[All Fields] OR normality[All Fields]) AND (population[MeSH Terms] OR population[All Fields] OR "population groups"[MeSH Terms])) OR ((health[MeSH Terms] OR health[All Fields] OR normality[All Fields]) AND individual[All Fields]) OR (reference[All Fields] AND individual[All Fields]) OR (reference[All Fields] AND ("population groups"[MeSH Terms] OR (population[All Fields] AND group[All Fields]) OR "population group"[All Fields] OR group[All Fields])) OR (reference[All Fields] AND cohort[All Fields]) OR (healthy[All Fields] AND cohort[All Fields]) OR (normal[All Fields] AND cohort[All Fields]) OR (cardio-healthy[All Fields] AND (population[MeSH Terms] OR population[All Fields])))

>II. EMBASE SEARCH (SEARCH DATE: 17-I0-2017, RESULTS: N = 340)

1. reference values.mp. or reference value|

2. $99^{\text {th }}$ centile.mp.

3. clinical decision limit.mp.

4. upper reference limit.mp.

5. $99^{\text {th }}$ percentile.mp.

6. 1 or 2 or 3 or 4 or 5

7. hs-cTn.mp.

8. hstnt.mp.

9. hstni.mp.

10. hs-cTnT.mp.

11. hs-cTnI.mp.

12. hsctnt.mp.

13. hsctni.mp.

14. ctnt.mp. 
15. ctni.mp.

16. troponin $\mathrm{I} /$ or troponin.mp. or troponin/ or troponin $\mathrm{T} /$

17. high sensitivity troponin.mp.

18. 7 or 8 or 9 or 10 or 11 or 12 or 13 or 14 or 15 or 16 or 17

19. 6 and 18

20. reference population.mp.

21. population group.mp. or population group/

22. normal population.mp.

23. healthy population.mp.

24. healthy individual.mp.

25. normality population.mp.

26. reference indivdual.mp.

27. reference group.mp.

28. reference cohort.mp.

29. healthy cohort.mp.

30. normal cohort.mp.

31. cardio-healthy population.mp.

32. 20 or 21 or 22 or 23 or 24 or 25 or 26 or 27 or 28 or 29 or 30 or 31

33. 19 and 32

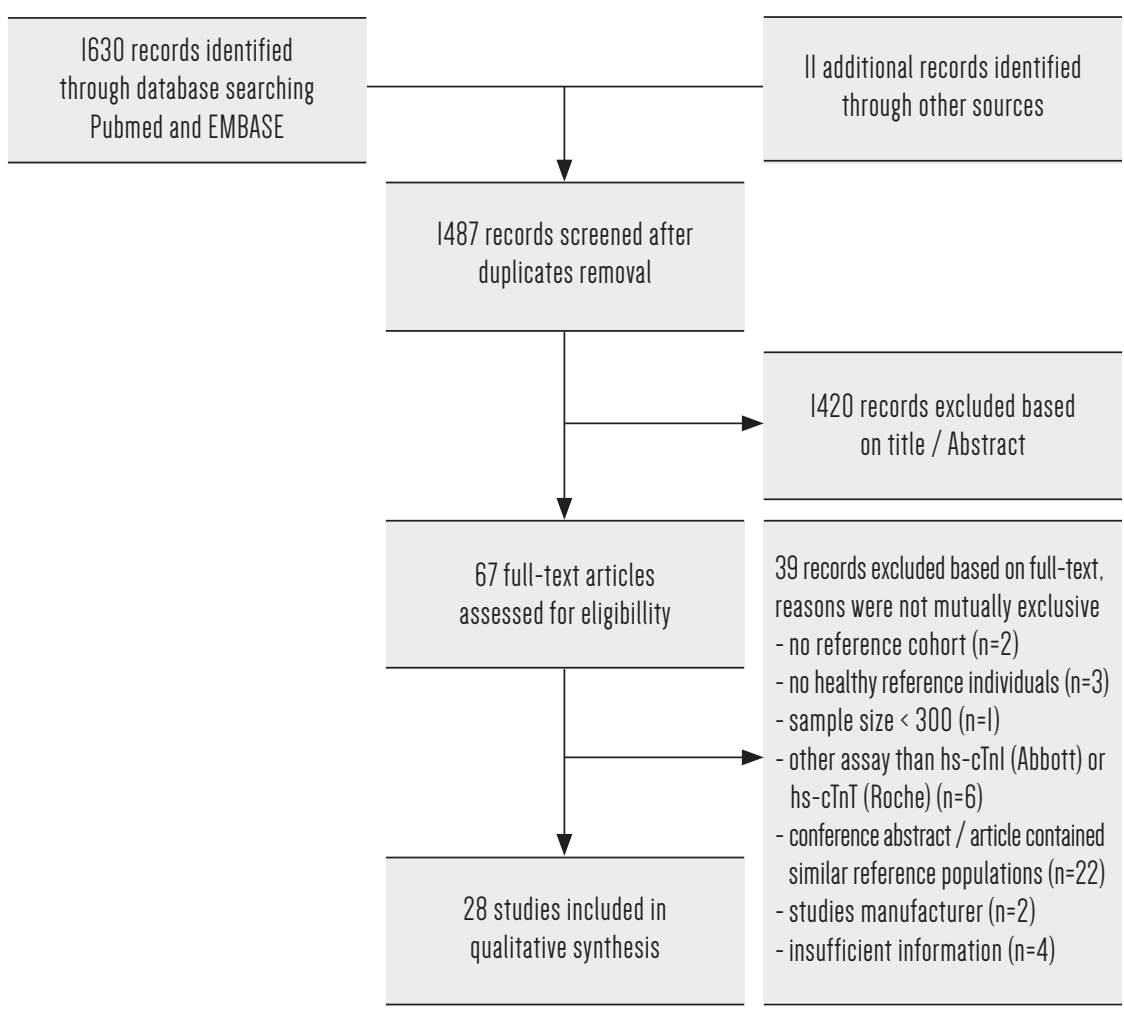

Supplemental 

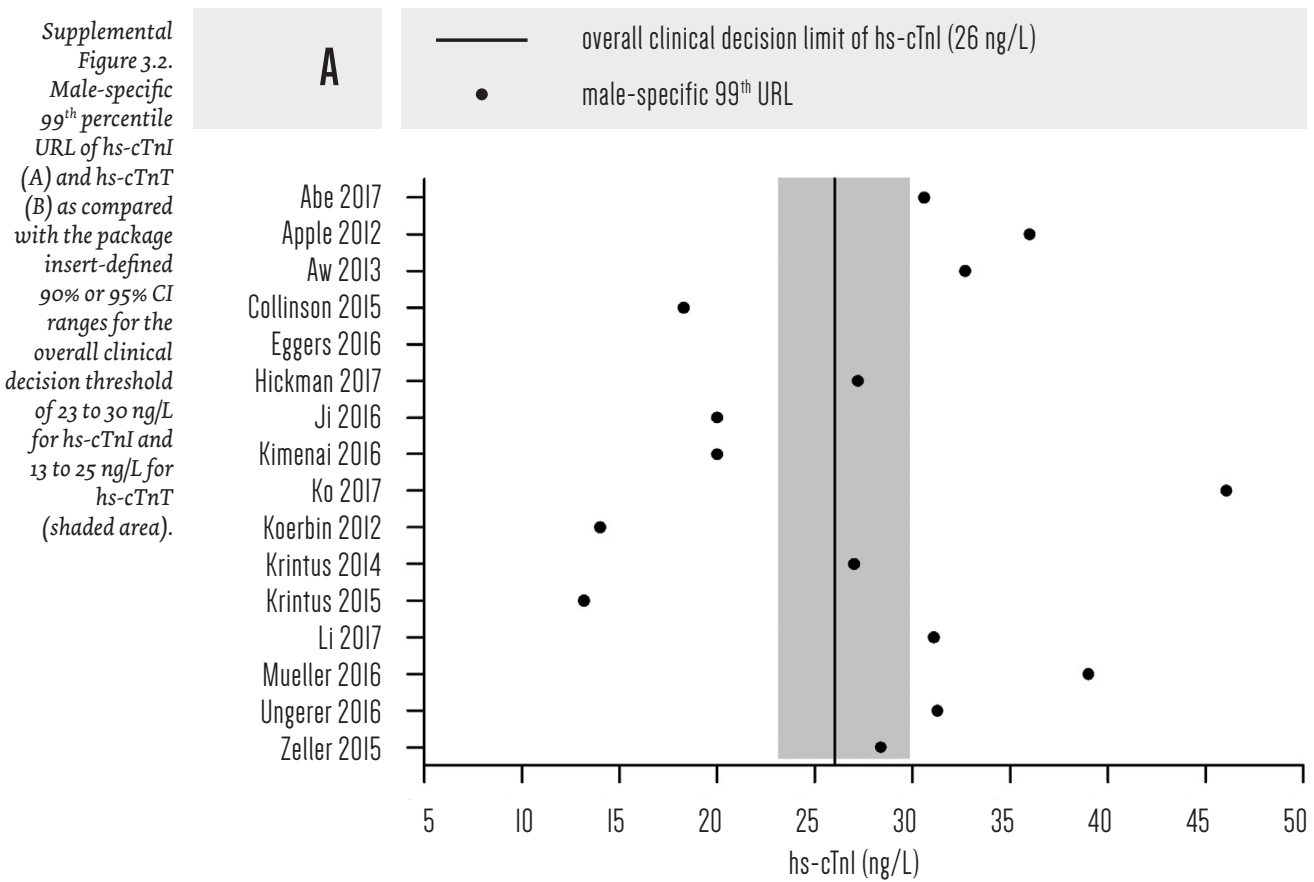

B
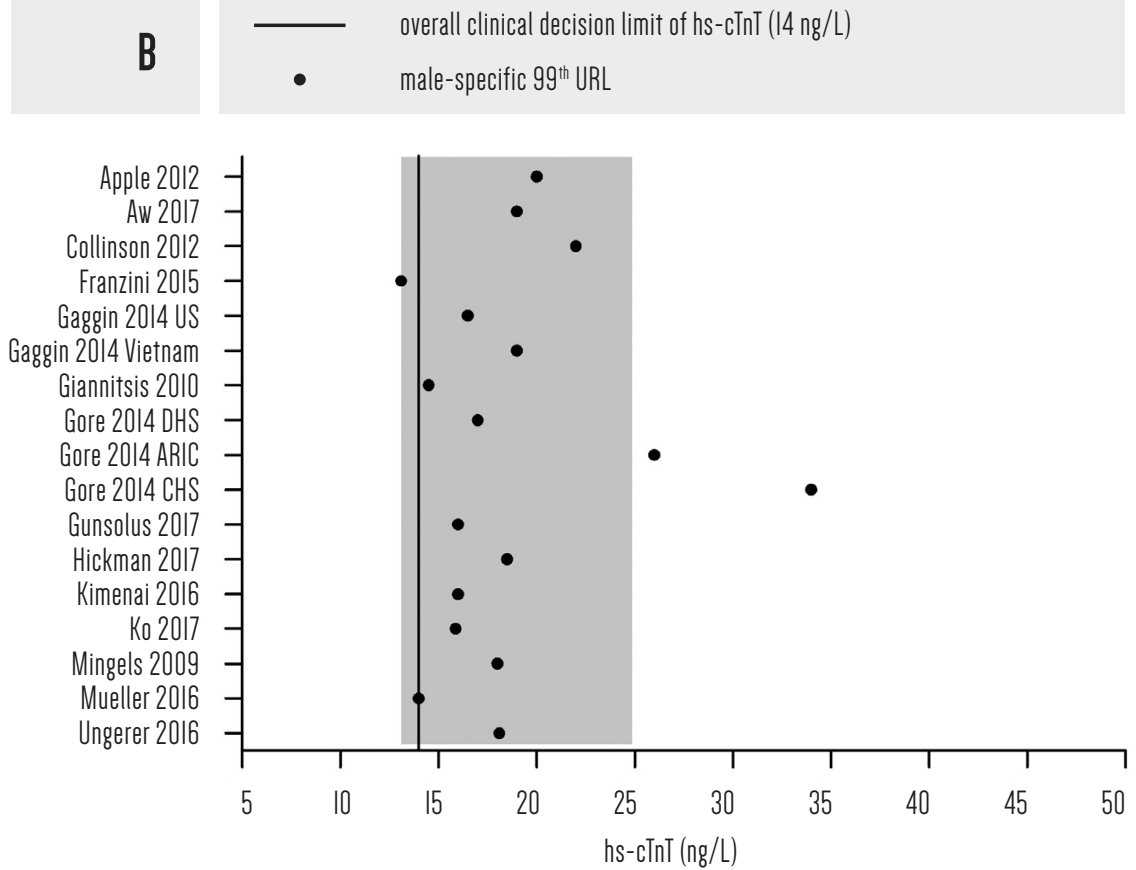
A
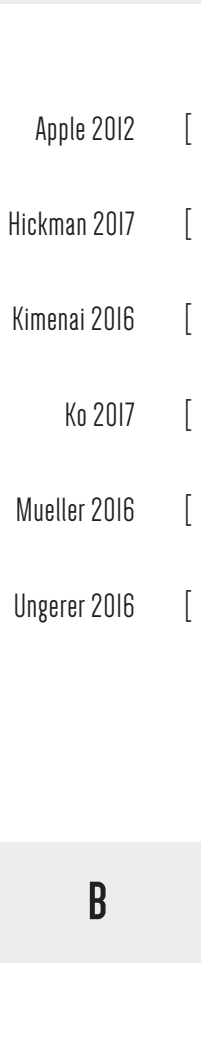

\section{Apple $2012 \quad[$}

Hickman 2017

Kimenai $2016 \quad$ [

Ko 2017 [

Mueller 2016

Ungerer 2016
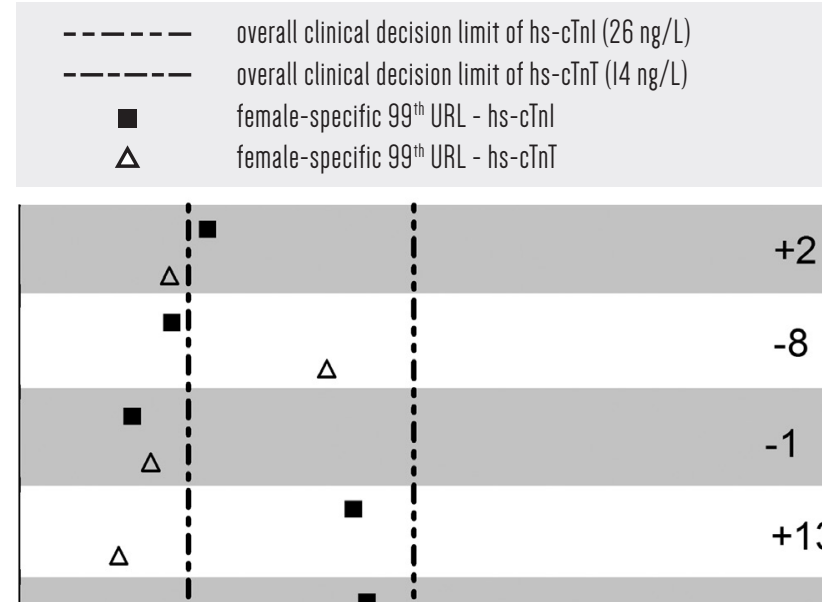

$-8$

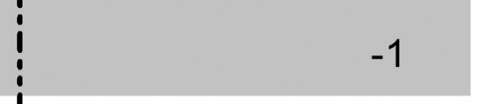

$\Delta$

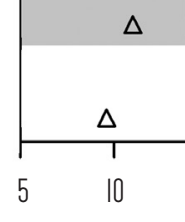

Supplemental

Figure 3.3.

Direct comparison offemale-specific $99^{\text {th }}$ percentile URL (A) and male-specific $99^{\text {th }}$ percentile URL (B) for hs-cTnI and hs-cTnT derived from a single reference population. The right column shows the absolute numeric differences between $99^{\text {th }}$ percentiles values of hs-cTnI and hs-cTnT from a single reference population.
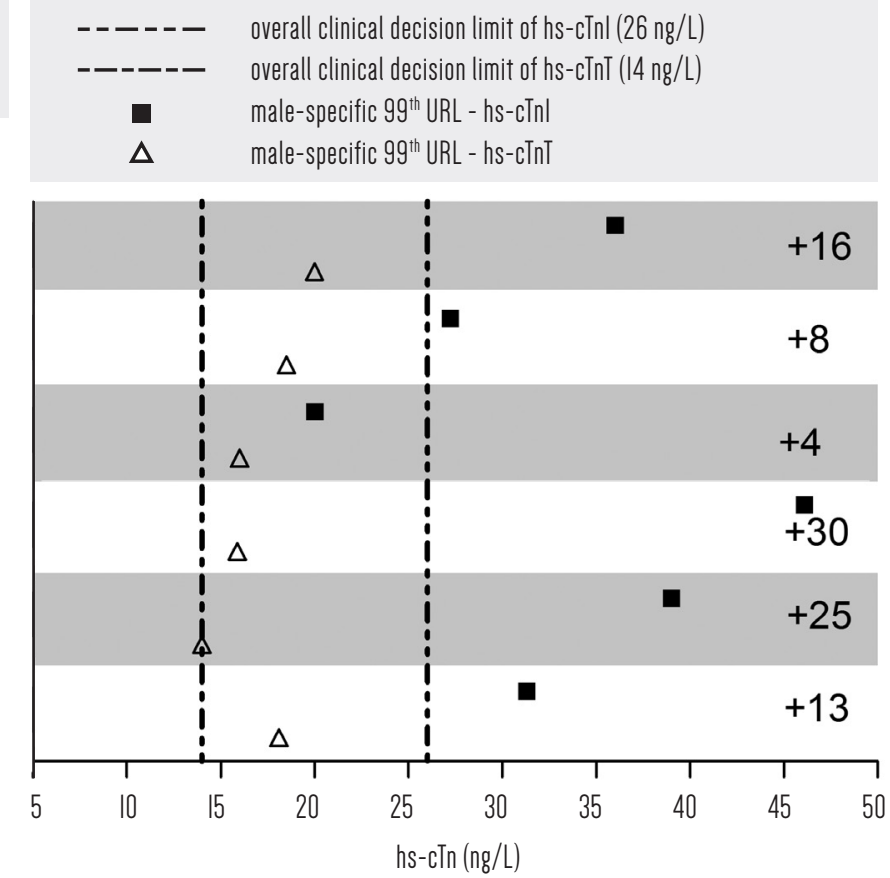


\begin{tabular}{|c|c|c|c|c|c|c|c|}
\hline \multirow{4}{*}{$\begin{array}{r}\text { Supplemental } \\
\text { Table } 3.1 . \\
\text { Sex-specific } \\
\text { and overall } 9 g^{\text {th }} \\
\text { percentile URL } \\
\text { of hs-cTnI and } \\
\text { hs-cTnT from a } \\
\text { single reference } \\
\text { population }(n=6) \text {. }\end{array}$} & Publication & $\begin{array}{l}\text { Female } \\
\text { (n) }\end{array}$ & $\begin{array}{l}\text { Female-specific } \\
99^{\text {th }} \text { percentile } \\
\text { (ng/L) }\end{array}$ & $\begin{array}{l}\text { Male } \\
\text { (n) }\end{array}$ & $\begin{array}{l}\text { Male-specific } \\
9^{\text {th }} \text { percentile } \\
\text { (ng/L) }\end{array}$ & $\begin{array}{l}\text { Reference } \\
\text { cohort } \\
\text { (n) }\end{array}$ & $\begin{array}{l}\text { Overall } \\
99^{\text {th }} \text { percentile } \\
\text { (ng/L) }\end{array}$ \\
\hline & \multicolumn{7}{|l|}{ hs-cTnl } \\
\hline & $\begin{array}{l}\text { Apple } \\
\text { et al. 2012। }\end{array}$ & 252 & 15 & 273 & 36 & 525 & $23[16-63]$ \\
\hline & $\begin{array}{l}\text { Hickman } \\
\text { et al. } 2017^{2}\end{array}$ & 226 & 13 & 222 & 27 & 484 & 14 \\
\hline & $\begin{array}{l}\text { Kimenai } \\
\text { et al. } 2016^{3}\end{array}$ & 806 & II (8-13) & 729 & $20(14-22)$ & 1535 & $13(||-18)$ \\
\hline & $\begin{array}{l}\text { Ko } \\
\text { et al. } 2017^{4}\end{array}$ & 338 & $23[17-29]$ & 303 & 46 [26-60] & 641 & $32[28-56]$ \\
\hline & $\begin{array}{l}\text { Mueller } \\
\text { et al. } 2016^{5}\end{array}$ & 143 & 24 & 259 & 39 & 402 & $29(|5-| 0 \mid)$ \\
\hline & $\begin{array}{l}\text { Ungerer } \\
\text { et al. } 2016^{6}\end{array}$ & 705 & $20[10-52]$ & 1299 & 31 [25-58] & 2004 & 29 [23-41] \\
\hline & \multicolumn{7}{|l|}{ hs-cTnT } \\
\hline & $\begin{array}{l}\text { Apple } \\
\text { et al. 2012! }\end{array}$ & 252 & 13 & 273 & 20 & 525 & $15[13-28]$ \\
\hline & $\begin{array}{l}\text { Hickman } \\
\text { et al. } 2017^{2}\end{array}$ & 262 & 21 & 228 & 19 & 490 & 20 \\
\hline & $\begin{array}{l}\text { Kimenai } \\
\text { et al. } 2016^{3}\end{array}$ & 806 & $12(10-14)$ & 729 & $16(15-17)$ & 1535 & $15(13-16)$ \\
\hline $\begin{array}{l}\text { The } 99^{\text {th }} \text { percentile } \\
\text { URL are reported } \\
\text { in } n g / L \text {, and if }\end{array}$ & $\begin{array}{l}\text { Ko } \\
\text { et al. } 2017^{4}\end{array}$ & 338 & $10[9-15]$ & 303 & 16 [I3-23] & 641 & $14[13-20]$ \\
\hline $\begin{array}{l}\text { determined, they } \\
\text { are presented }\end{array}$ & $\begin{array}{l}\text { Mueller } \\
\text { et al. } 2016^{5}\end{array}$ & 143 & $\|$ & 259 & 14 & 402 & $12(9-20)$ \\
\hline $\begin{array}{l}\text { with their } 90 \% \text { CI } \\
\text { [] or } 95 \% \text { CI(), } \\
\text { respectively. }\end{array}$ & $\begin{array}{l}\text { Ungerer } \\
\text { et al. } 2016^{6}\end{array}$ & 705 & $10[9-11]$ & 1299 & 18 [|6-20] & 2004 & 16 [15-18] \\
\hline
\end{tabular}




\begin{tabular}{|c|c|c|c|c|c|c|}
\hline Publication & $\begin{array}{l}\text { Female } \\
\text { (n) }\end{array}$ & $\begin{array}{l}\text { Female-specific } \\
99^{\text {th }} \text { percentile } \\
(\mathrm{ng} / \mathrm{L})\end{array}$ & $\begin{array}{l}\text { Male } \\
\text { (n) }\end{array}$ & $\begin{array}{l}\text { Male-specific } \\
99^{\text {th }} \text { percentile } \\
\text { (ng/L) }\end{array}$ & $\begin{array}{l}\text { Reference } \\
\text { cohort } \\
\text { (n) }\end{array}$ & $\begin{array}{l}\text { Overall } \\
9 g^{\text {th }} \text { percentile } \\
\text { (ng/L) }\end{array}$ \\
\hline $\begin{array}{l}\text { Abe } \\
\text { et al. } 2018^{7}\end{array}$ & 385 & $18(12-23)$ & 313 & $31(17-53)$ & 698 & $23(17-37)$ \\
\hline $\begin{array}{l}\text { Aw } \\
\text { et al. } 2013^{8}\end{array}$ & 523 & 18 [II-26] & 597 & 33 [21-48] & $\| 20$ & 26 [20-33] \\
\hline $\begin{array}{l}\text { Collinson } \\
\text { et al. } 2015^{9}\end{array}$ & 195 & 10 & |7| & 18 & 366 & $12(6-21)$ \\
\hline $\begin{array}{l}\text { Eggers } \\
\text { et al. 2016 } 10\end{array}$ & 255 & $25(||-3 \mid)$ & 266 & 42 (22-73) & 521 & $37(2 \mid-45)$ \\
\hline $\begin{array}{l}\text { Ji } \\
\text { et al. 2016" }\end{array}$ & 428 & 19 [II-4|] & 426 & $20[15-69]$ & 854 & $18[14-35]$ \\
\hline $\begin{array}{l}\text { Koerbin } \\
\text { et al. 2012 }\end{array}$ & 221 & $\|[||-\mid 2]$ & 276 & $14[13-14]$ & 497 & $14[13-14]$ \\
\hline $\begin{array}{l}\text { Krintus } \\
\text { et al. 2014 }{ }^{13}\end{array}$ & 776 & II [10-15] & 993 & $27[20-67]$ & 1769 & $19[14-25]$ \\
\hline $\begin{array}{l}\text { Krintus } \\
\text { et al. } 2015^{14}\end{array}$ & 354 & $9[7-16]$ & 280 & 13 & 634 & II [8-16] \\
\hline $\begin{array}{l}\text { Krintus } \\
\text { et al. } 2016^{15}\end{array}$ & 208 & not reported & 200 & not reported & 408 & $6[6-8]$ \\
\hline $\begin{array}{l}\text { Li } \\
\text { et al. } 2017^{16}\end{array}$ & 754 & 23 & $73 \mid$ & 31 & 1485 & 28 \\
\hline $\begin{array}{l}\text { Zeller } \\
\text { et al. } 2015^{17}\end{array}$ & 1316 & $12(9-21)$ & 1292 & $28(21-42)$ & 2608 & $21(18-29)$ \\
\hline
\end{tabular}

Supplemental Table 3.2. Sex-specific and overall $99^{\text {th }}$ percentile URL of $h s-c \operatorname{TnI}(n=11)$.

The $99^{\text {th }}$ percentile URL are reported in $n g / L$, and if determined, they are presented with their 90\% CI [ ] or $95 \% \mathrm{CI}$ ( ), respectively. 


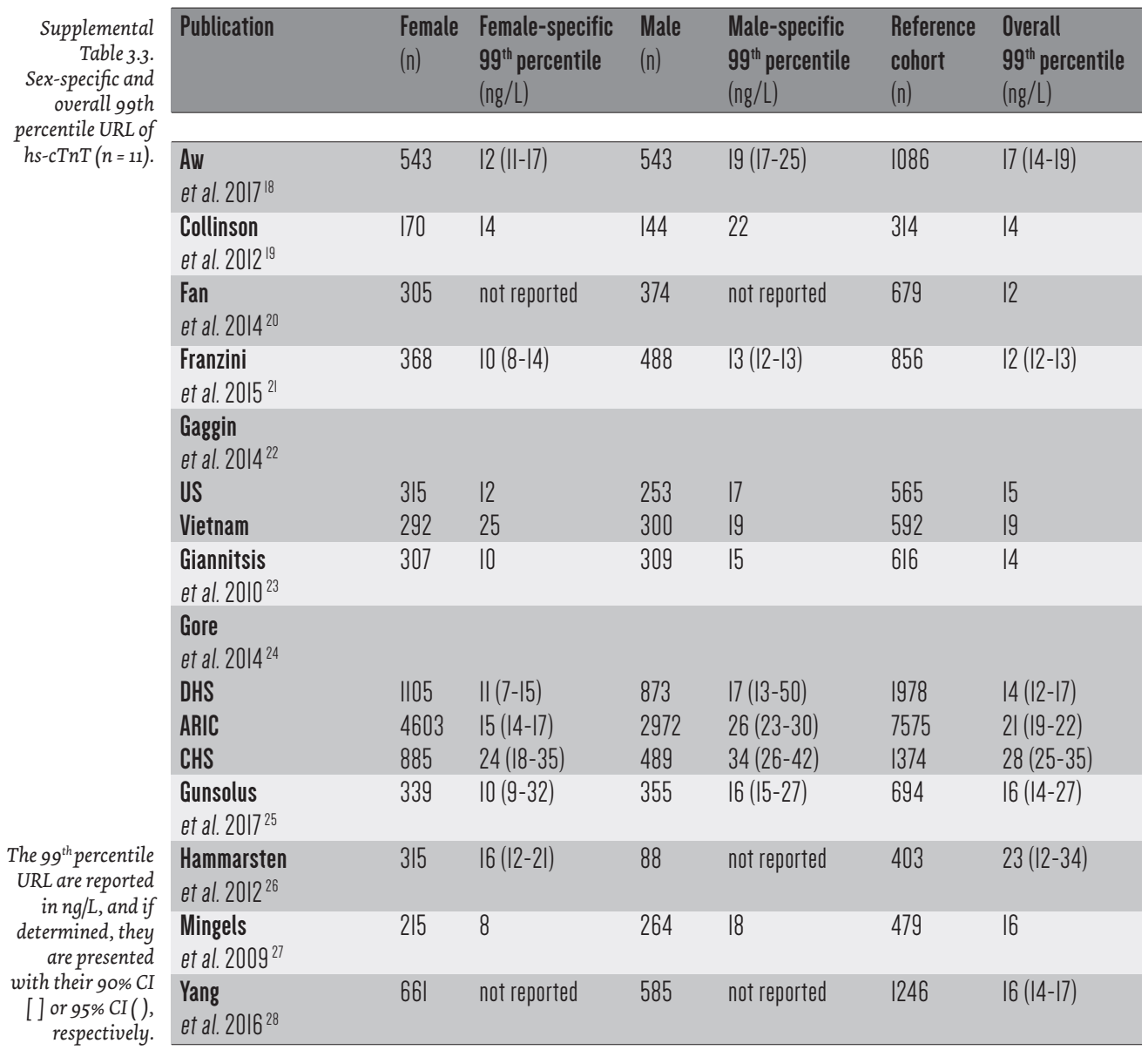




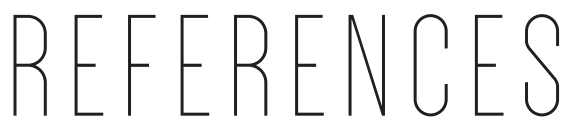

1. Apple FS, Ler R, Murakami MM. Determination of 19 cardiac troponin I and T assay $99^{\text {th }}$ percentile values from a common presumably healthy population. Clin Chem 2012;58:1574-81.

2. Hickman PE, Koerbin G, Potter JM, Abhayaratna WP. Statistical considerations for determining high-sensitivity cardiac troponin reference intervals. Clin Biochem 2017;50:502-5.

3. Kimenai DM, Henry RM, van der Kallen CJ, Dagnelie PC, Schram MT, Stehouwer CD, et al. Direct comparison of clinical decision limits for cardiac troponin $\mathrm{T}$ and I. Heart 2016;102:610-6.

4. Ko DH, Jeong TD, Cho EJ, Lim J, Ji M, Lee K, et al. The $99^{\text {th }}$ percentile values of six cardiac troponin assays established for a reference population using strict selection criteria. Clin Chim Acta 2017;464:1-5.

5. Mueller T, Egger M, Leitner I, Gabriel C, Haltmayer M, Dieplinger B. Reference values of galectin-3 and cardiac troponins derived from a single cohort of healthy blood donors. Clin Chim Acta 2016;456:19-23.

6. Ungerer JP, Tate JR, Pretorius CJ. Discordance with 3 Cardiac Troponin I and T assays: Implications for the $99^{\text {th }}$ Percentile Cutoff. Clin Chem 2016;62:1106-14.

7. Abe N, Tomita K, Teshima M, Kuwabara M, Sugawa S, Hinata N, et al. Distribution of cardiac troponin I in the Japanese general population and factors influencing its concentrations. J Clin Lab Anal 2018;32(3).

8. Aw TC, Phua SK, Tan SP. Measurement of cardiac troponin I in serum with a new highsensitivity assay in a large multi-ethnic Asian cohort and the impact of gender. Clin Chim Acta 2013;422:26-8.

9. Collinson PO, Gaze D, Goodacre S. The clinical and diagnostic performance characteristics of the high sensitivity Abbott cardiac troponin I assay. Clin Biochem 2015;48:275-81.

10. Eggers KM, Apple FS, Lind L, Lindahl B. The applied statistical approach highly influences the $99^{\text {th }}$ percentile of cardiac troponin I. Clin Biochem 2016;49:1109-12.

11. Ji M, Moon HW, Hur M, Yun YM. Determination of high-sensitivity cardiac troponin I $99^{\text {th }}$ percentile upper reference limits in a healthy Korean population. Clin Biochem 2016;49:756-61.

12. Koerbin G, Tate J, Potter JM, Cavanaugh J, Glasgow N, Hickman PE. Characterisation of a highly sensitive troponin I assay and its application to a cardio-healthy population. Clin Chem Lab Med 2012;50:871-8. 
13. Krintus M, Kozinski M, Boudry P, Capell NE, Koller U, Lackner K, et al. European multicenter analytical evaluation of the Abbott ARCHITECT STAT high sensitive troponin I immunoassay. Clin Chem Lab Med 2014;52:1657-65.

14. Krintus M, Kozinski M, Boudry P, Lackner K, Lefevre G, Lennartz L, et al. Defining normality in a European multinational cohort: Critical factors influencing the $99^{\text {th }}$ percentile upper reference limit for high sensitivity cardiac troponin I. Int J Cardiol 2015;187:256-63.

15. Krintus M, Kozinski M, Fabiszak T, Kuligowska-Prusinska M, Laskowska E, Lennartz L, et al. Impact of lipid markers and high-sensitivity C-reactive protein on the value of the $99^{\text {th }}$ percentile upper reference limit for high-sensitivity cardiac troponin I. Clin Chim Acta 2016;462:193-200.

16. Li S, Zuo Y, Huang W. Establishment of a reference interval for high-sensitivity cardiac troponin I in healthy adults from the Sichuan area. Medicine (Baltimore) 2017;96:e6252.

17. Zeller T, Ojeda F, Brunner FJ, Peitsmeyer P, Munzel T, Binder H, et al. High-sensitivity cardiac troponin I in the general population--defining reference populations for the determination of the $99^{\text {th }}$ percentile in the Gutenberg Health Study. Clin Chem Lab Med 2015;53:699-706.

18. Aw T, Phua S, Lam C, Wong M. What are normal high-sensitivity troponin-T values in a large multi-ethnic Asian population? Blood, Heart and Circulation 2017;1:1-4.

19. Collinson PO, Heung YM, Gaze D, Boa F, Senior R, Christenson R, Apple FS. Influence of population selection on the $99^{\text {th }}$ percentile reference value for cardiac troponin assays. Clin Chem 2012;58:219-25.

20. Fan L, Yu P, Yu S, Gu Y, Zong M, Cai Y, Liu Z. Age-specific $99^{\text {th }}$ percentile cutoff of high-sensitivity cardiac troponin $\mathrm{T}$ for early prediction of non-ST-segment elevation myocardial infarction (NSTEMI) in middle-aged patients. J Clin Lab Anal 2014;28:10-5.

21. Franzini M, Lorenzoni V, Masotti S, Prontera C, Chiappino D, Latta DD, et al. The calculation of the cardiac troponin $\mathrm{T} 99^{\text {th }}$ percentile of the reference population is affected by age, gender, and population selection: a multicenter study in Italy. Clin Chim Acta 2015;438:376-81.

22. Gaggin HK, Dang PV, Do LD, deFilippi CR, Christenson RH, Lewandrowski EL, et al. Reference interval evaluation of high-sensitivity troponin $\mathrm{T}$ and $\mathrm{N}$-terminal B-type natriuretic peptide in Vietnam and the US: The North South East West Trial. Clin Chem 2014;60:758-64.

23. Giannitsis E, Kurz K, Hallermayer K, Jarausch J, Jaffe AS, Katus HA. Analytical validation of a high-sensitivity cardiac troponin T assay. Clin Chem 2010;56:254-61.

24. Gore MO, Seliger SL, Defilippi CR, Nambi V, Christenson RH, Hashim IA, et al. Ageand sex-dependent upper reference limits for the high-sensitivity cardiac troponin T assay. J Am Coll Cardiol 2014;63:1441-8.

25. Gunsolus IL, Jaffe AS, Sexter A, Schulz K, Ler R, Lindgren B, et al. Sex-specific $99^{\text {th }}$ percentiles derived from the AACC Universal Sample Bank for the Roche Gen $5 \mathrm{cTnT}$ assay: Comorbidities and statistical methods influence derivation of reference limits. Clin Biochem 2017;50:1073-7. 
26. Hammarsten O, Fu ML, Sigurjonsdottir R, Petzold M, Said L, Landin-Wilhelmsen $\mathrm{K}$, et al. Troponin $\mathrm{T}$ percentiles from a random population sample, emergency room patients and patients with myocardial infarction. Clin Chem 2012;58:628-37.

27. Mingels A, Jacobs L, Michielsen E, Swaanenburg J, Wodzig W, van Dieijen-Visser M. Reference population and marathon runner sera assessed by highly sensitive cardiac troponin $\mathrm{T}$ and commercial cardiac troponin $\mathrm{T}$ and $\mathrm{I}$ assays. Clin Chem 2009;55:101-8.

28. Yang S, Huai W, Qiao R, Cui L, Liu G, Wu J, et al. Age and Gender Tailored Cutoff Value of hs-cTnT Contributes to Rapidly Diagnose Acute Myocardial Infarction in Chest Pain Patients. Clin Lab 2016;62:1451-9. 


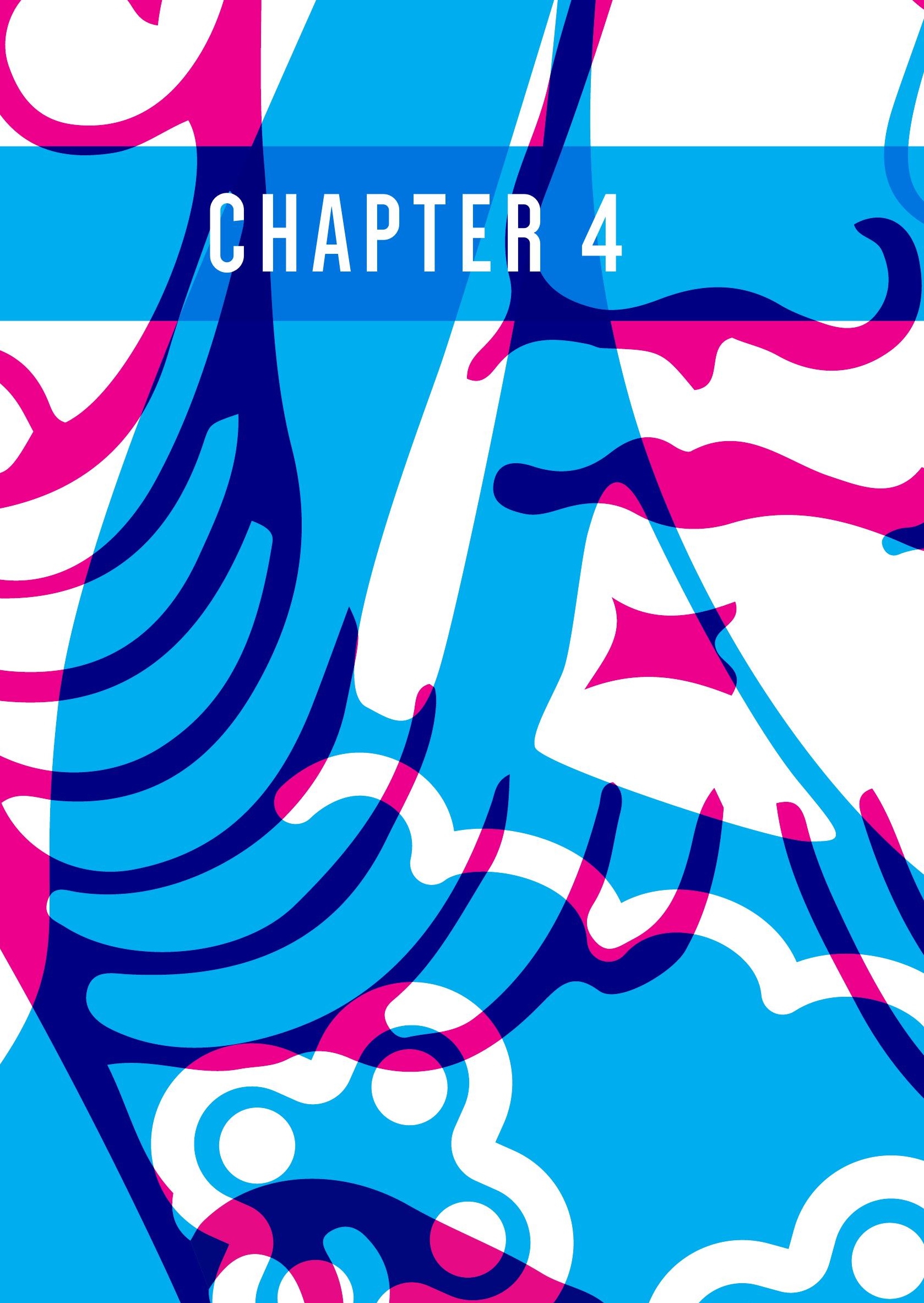



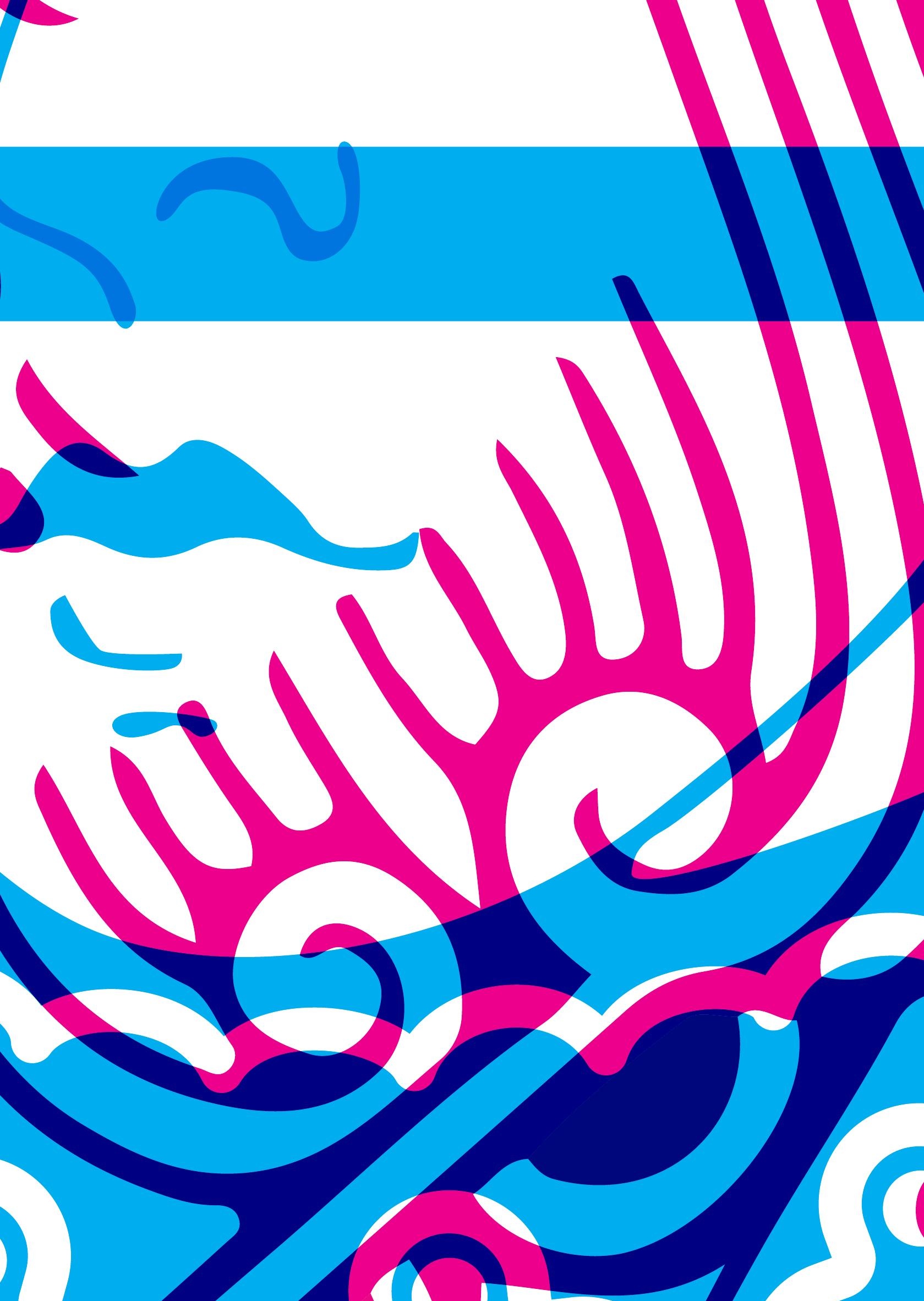


\section{CHAPTER 4}

SEX-RELATED ASPECTS

OF BIOMARKERS IN

CARDIAC DISEASE

Published as book chapter in "Sex-Specific Analysis of Cardiovascular Function"

Alma M Mingels and Dorien M Kimenai

Editors: Peter LM Kerkhof and Virginia M Miller

Advances in Experimental Medicine and Biology 2018; 1065:545-564 


\section{ABSTRACT}

Biomarkers play an important role in the clinical management of cardiac care. In particular, cardiac troponins ( $\mathrm{cTn}$ ) and natriuretic peptides are the cornerstones for the diagnosis of acute myocardial infarction (AMI) and for the diagnosis of heart failure (HF), respectively. Current guidelines do not make a distinction between women and men.

However, the commonly used "one size fits all" algorithms are topic of debate to improve assessment of prognosis, particularly in women. Due to the highsensitivity assays (hs-cTn), lower cTn levels (and $99^{\text {th }}$ percentile upper reference limits) were observed in women as compared with men. Sex-specific diagnostic thresholds may improve the diagnosis of AMI in women, though clinical relevance remains controversial and more trials are needed. Also other diagnostics aspects are under investigation, like combined biomarkers approach and rapid measurement strategies.

For the natriuretic peptides, previous studies observed higher concentrations in women than in men, especially in premenopausal women who might benefit from the cardio-protective actions. Contrary to hs-cTn, natriuretic peptides are particularly incorporated in the ruling-out algorithms for the diagnosis of HF and not ruling-in. Clinical relevance of sex differences here seems marginal, as clinical research has shown that negative predictive values for ruling-out HF were hardly effected when applying a universal diagnostic threshold that is independent from sex or other risk factors.

Apart from the diagnostic issues of AMI in women, we believe that in the future most sex-specific benefits of cardiac biomarkers can be obtained in patient follow-up (guiding therapy) and prognostic applications, fitting modern ideas on preventive and personalized medicine. 


\section{INTRODUCTION}

Biomarkers play an important role in the clinical management of cardiac care and risk stratification for cardiovascular diseases (CVD) ${ }^{1,2}$ and heart failure. ${ }^{3,4}$ Although over the last decades the number of deaths attributable to CVD has declined globally ${ }^{5}$, the reduction on CVD-related deaths is less pronounced for women than men. ${ }^{6}$ With respect to sex-specific analysis, the common cardiac care guidelines do not make a distinction between women and men on the use of cardiac biomarkers. ${ }^{1-4}$ However, the awareness has been raised that sex inequalities, and hence, the use of the "one size fits all" algorithms that comprises cardiac biomarkers, may hamper the diagnosis and treatment in women. ${ }^{1,7}$

A number of biomarkers such as lactate dehydrogenase, creatine kinase (CK), or creatine kinase muscle-brain (CKMB) type were used in the past for the diagnosis of acute myocardial infarction (AMI) (Figure 4.1). Though most of these biomarkers

Figure 4.1.

History of cardiac

biomarkers for

the diagnosis of acute myocardial infarction.

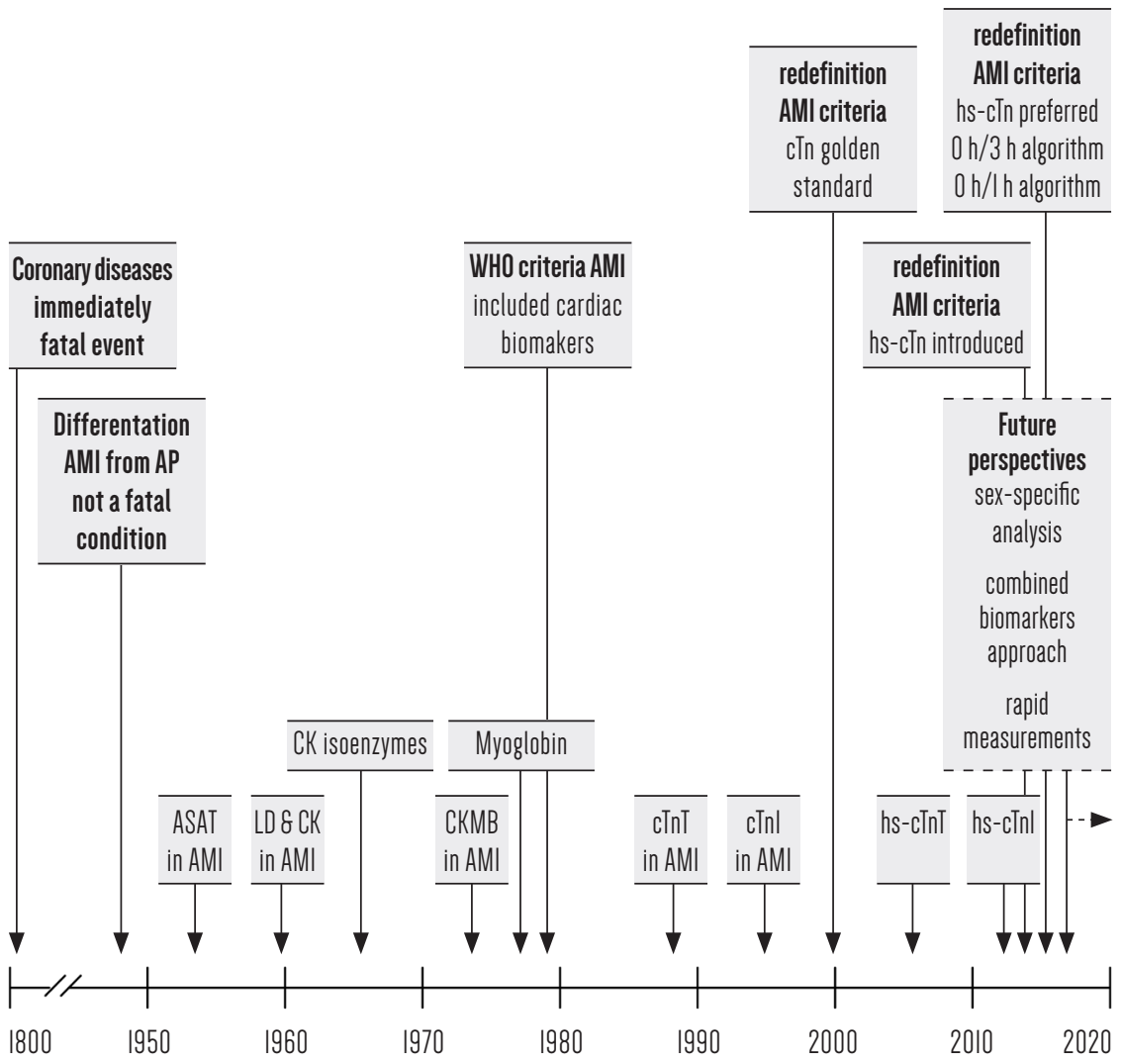


are run in every clinical laboratory today, also for other purposes, their main disadvantage is the lack in cardiac specificity and so none of them are preferred anymore in the diagnosis of cardiac diseases..$^{1,2,8} \mathrm{At}$ this point, cardiac troponins (cTn) are, due to their outperformance on cardiac specificity, incorporated in common guidelines and clinical practice for diagnosis, risk stratification, and prognosis of acute coronary syndrome (ACS). ${ }^{1,2}$

That no sex-based differences are made in cTn algorithms can be explained from a historical point of view, since the "older" assays were not able to detect any sex differences. Due to the analytical improvement of these laboratory assays over the last years, nowadays, even basal levels of cTn in healthy individuals can be measured. ${ }^{9}$ This improvement resulted in the observation that disparities between women and men on cTn levels became apparent. ${ }^{10}$ Hence, the technical progress has led to the ongoing debate whether the "one size fits all" strategy is sufficiently sensitive for women.

Also regarding the use of natriuretic peptides no sex-based differences are incorporated in the guidelines and clinical practice, ${ }^{3,4}$ despite the fact that it has clearly been shown that natriuretic peptide concentrations differ between men and women. ${ }^{11,12}$

In this chapter we will provide an overview of the current knowledge and existing gaps on sex differences of cardiac biomarkers. We focus on cTn as the gold standard for the diagnosis of AMI and we focus on natriuretic peptides (B-type natriuretic peptic, BNP and N-terminal proBNP, NT-proBNP) in the area of diagnosis and prognosis of heart failure (HF).

\section{CARDIAC TROPONINS}

\section{$>$ THE BASICS OF CARDIAC TROPONINS}

The contractile apparatus of cardiac and skeletal muscle cells consists of thick and thin filaments that slide along each other upon muscle contraction and relaxation. Thick filaments are built up from myosin; thin filaments are built up from actin with tropomyosin wrapped around and at regular sites a troponin complex of troponin $\mathrm{T}$ $(\mathrm{TnT})$, troponin I (TnI), and troponin C (TnC).

TnT, TnI, and TnC are tropomyosin binding, inhibition, and calcium binding components, respectively. ${ }^{13}$ Upon stimulation of the myocytes, calcium ions are released and bound to $\mathrm{TnC}$, and subsequently there is a conformational change of the troponin complex, resulting in accessible binding sites between actin and myosin and leading to contraction of the muscles. TnT and TnI isoforms are characterized by a cardiac-specific N-terminal extension, while $\mathrm{TnC}$ in cardiac and slow skeletal 
muscle is similar (Swiss-Prot, P19429, P45379, and P63316, respectively), which makes them highly valuable for detection of cardiac injury and thus for the management of cardiac diseases. It has been estimated that around 5-10\% of the total cTnT and cTnI content is present free and unbound in the cytosol, but this statement has been questioned by others. TnC, on the other hand, is not a cardiac-specific biomarker and will be therefore left out of consideration in this chapter.

After cardiac injury, e.g., in acute myocardial infarction (AMI), cardiac troponin T ( $\mathrm{cTnT}$ ) and cardiac troponin I (cTnI) are released in the bloodstream and detectable for diagnostic and prognostic purposes. ${ }^{1,2}$ In AMI patients, a main peak is typically shown within one day after onset of symptoms of acute chest pain and remains elevated for approximately one week, as depicted in Figure 4.2. It was hypothesized that the first peak reflects the relatively fast release of cytosolic cTn, while the second broader peak represents the slower dissociation of cTn from the sarcomeres. If this is true, it is quite remarkable that cTnT and cTnI show a slight difference in their return to baseline. We believe this is due to differences between cTnT and cTnI clearance, as there are differences observed between cTnT and cTnI in patients with reduced kidney function ${ }^{14}$, both proteins differ in their biochemical composition and their molecular weights (cTnT and cTnI being $37 \mathrm{kDa}$ and $26 \mathrm{kDa}$, respectively), plus their susceptibility to degradation..$^{15,16}$

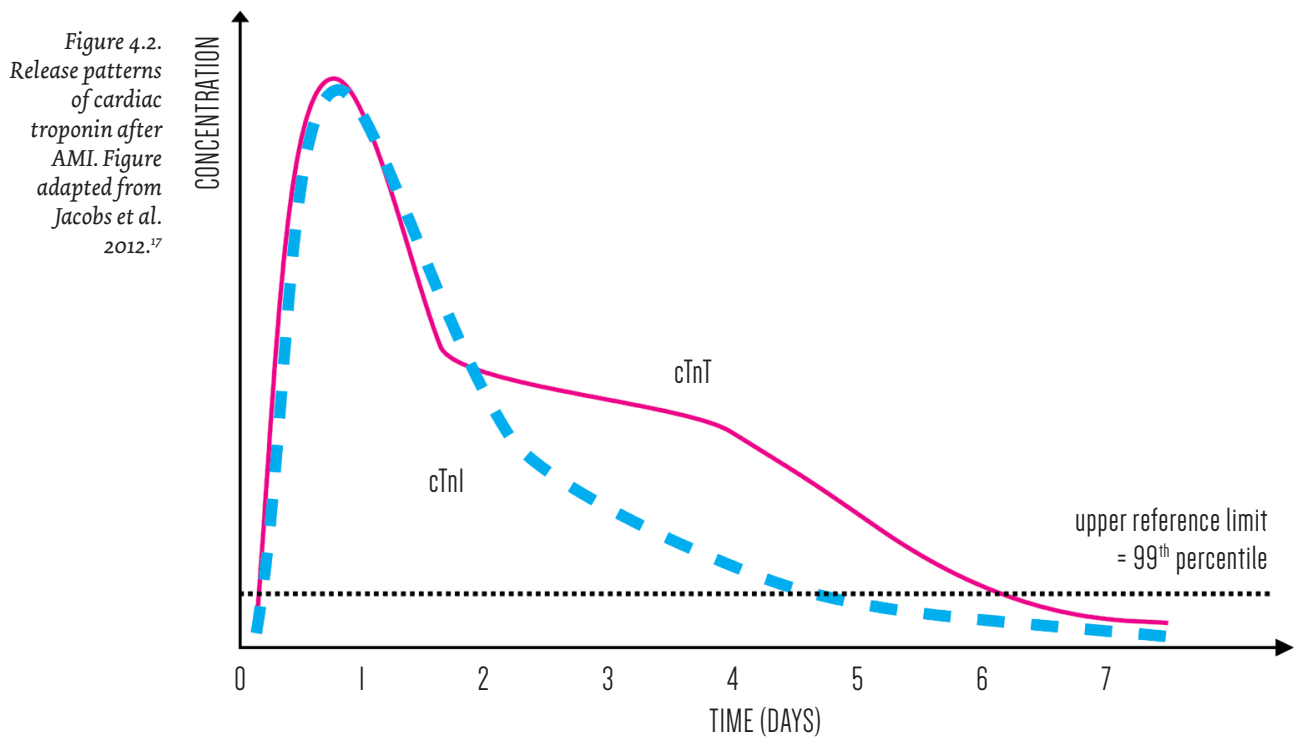




\section{CARDIAC TROPONINS IN HEALTHY WOMEN AND MEN}

In the past, due to the restriction of conventional cTn assays, clinicians and scientists could easily discriminate "patients with AMI" from "patients without AMI" by the ability to detect positive cTn levels in case of clinical cardiac injury. However, a higher prevalence of cTn levels, particularly cTnT, was also seen in patients with kidney diseases. ${ }^{18-20}$ Furthermore, also in particular subsets of individuals with several cardiac phenotypes like left ventricular hypertrophy (LVH) and congestive heart failure (CHF), elevated troponins were present. ${ }^{21}$

The prominent factor that shed new light on the thought that cTn are not only the result of clinical cardiac injury was the technical improvement of the cTn assays. At this point, using the so-called "high-sensitivity" assays, we now know that cTn levels are also present in apparently healthy individuals. ${ }^{9}$ In particular subgroups, and now to an even larger and more obvious extent, a higher prevalence of cTn elevations is observed, mainly in individuals who are at risk for cardiovascular events, like in individuals with diabetes mellitus, hypertension, and reduced kidney function. ${ }^{18,21-24}$ The current guidelines listed the conditions whereby elevated levels of cTn are observed other than AMI to consider for further diagnostic workup (Table 4.1). ${ }^{1}$

With the shift to more sensitive cTn assays, also sex inequalities of cTn levels in apparently healthy women and men became evident. ${ }^{10}$ Although it is nowadays well established that basal levels of cTn are significantly lower in women than men, the underlying mechanisms of these divergence are still not completely unraveled and understood, but these are most likely multifactorial. ${ }^{25}$ The pathogenesis of coronary artery disease (CAD) between women and men seems to differ. Women with CAD are more often presented with endothelial dysfunction than men, while men are more often present with more localized, i.e., less diffuse, coronary disease. ${ }^{26,27}$ The sex hormone estrogen seems to play a protective role in the pathogenesis of several cardiac pathologies. ${ }^{25}$ It turned out that estrogen attenuates the processes of atherosclerosis, $\mathrm{LVH}$, and cardiomyocyte apoptosis, possibly resulting into lower cTn levels in women than men. ${ }^{27-29}$ Another important contributor to sex-based differences of cTn levels seems to be the left ventricular (LV) anatomy. The LV mass differs between women and men, whereby men have a higher LV mass as compared with women..$^{30}$ Previous studies showed a strong relationship between LV mass and circulating cTn levels ${ }^{31,32}$, and the higher cardiac mass of men probably leads to more release of $\mathrm{cTn}$, which results into a higher basal circulating cTn levels in men than women. 
Table 4.1.

Conditions

or procedures whereby elevated

levels of cTn

are observed

other than AMI.

Adapted from

ESC guidelines

2016.

\section{Most frequent conditions}

Tachyarrhythmias

Heart failure

Critical illness (e.g., shock/ sepsis/ burns)

Myocarditis (incl. myocardial extension of endocarditis or pericarditis)

Takotsubo cardiomyopathy

Structural heart disease (e.g., aortic stenosis)

Aortic dissection

Pulmonary embolism, pulmonary hypertension

Renal dysfunction and associated cardiac disease

Other conditions or procedures

Coronary spasm

Acute neurological event (e.g., stroke or subarachnoid hemorrhage)

Cardiac contusion or cardiac procedures

(CABG, PCI, ablation, pacing, cardioversion, or endomyocardial biopsy)

Hypo- and hyperthyroidism

Infiltrative diseases (e.g. amyloidosis, hemochromatosis, sarcoidosis, scleroderma)

Myocardial drug toxicity or poisoning (e.g., doxorubicin, 5-fluorouracil, Herceptin, snake venoms)

Extreme endurance efforts

Rhabdomyolysis

\section{> CARDIAC TROPONINS FOR THE DIAGNOSIS OF ACUTE MYOCARDIAL INFARCTION}

The cornerstones in the diagnosis of AMI are the clinical presentation, electrocardiography (ECG) findings, and cardiac troponin measurements (Figure 4.3). For patients with ST-elevation myocardial infarction (STEMI), the ECG findings are the gold standard to establish AMI diagnosis. However, patients with non-STelevation myocardial infarction (NSTEMI) show no diagnostic or inconclusive ECG elevations, whereby the clinical presentation of the patient, in conjunction with cTn measurements, establishes the diagnosis of AMI.

The current guidelines require a significant "rise and/or fall" of high-sensitivity cardiac troponin $\mathrm{T}$ (hs-cTnT) or I (hs-cTnI) between serial measurements with at least one value above the $99^{\text {th }}$ percentile upper reference limit of hs-cTn from a healthy reference population. ${ }^{1,2}$ Although the ESC guidelines recommend this oh $/ 3 \mathrm{~h}$ algorithm, they incorporated also an oh/1h algorithm as alternative approach. ${ }^{1}$ The oh/1h algorithm handles other diagnostic thresholds for hs-cTnT and hs-cTnI, dependent of which hs-cTn assay is used. ${ }^{1}$ 


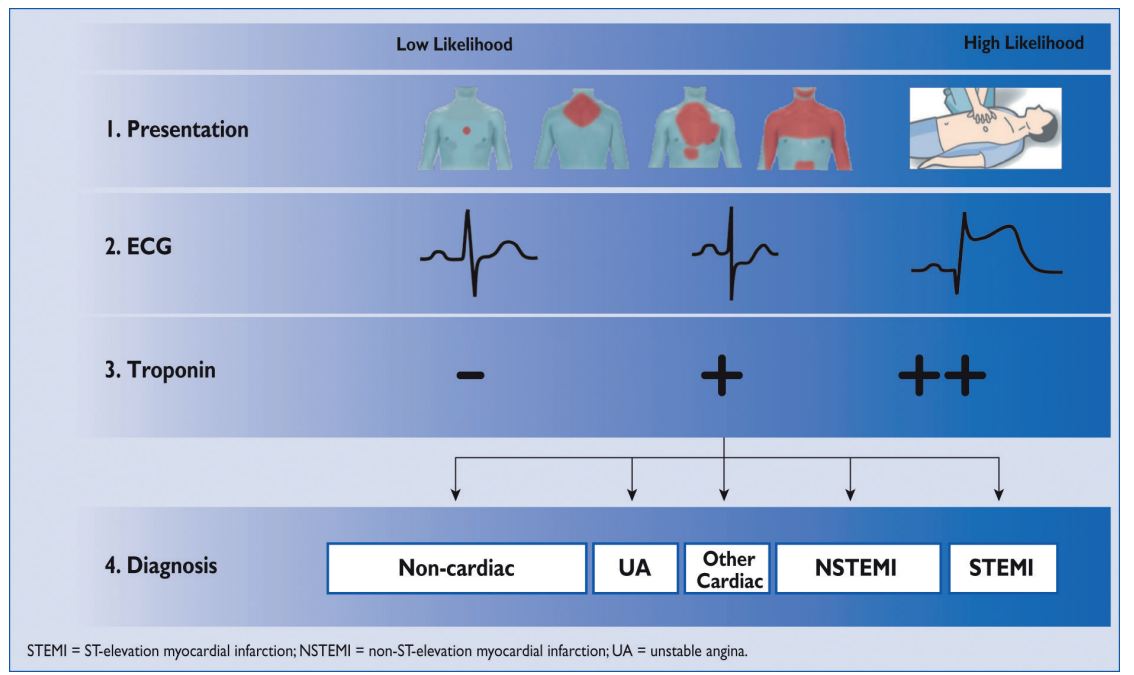

The diagnosis of AMI in women is particularly challenging, as women with suspected ACS are more likely to present with an atypical clinical presentation and/ or inconclusive ECG, which may hamper the diagnosis of AMI.33-35 Besides, women have a higher prevalence of unrecognized (silent) AMIs as compared with men (women 54\% vs men 33\%). ${ }^{36}$ All in all this may lead to worse prognosis after AMI in women, and therefore an optimized diagnostic cTn algorithm is of great clinical importance.

Over the last years, we may already have booked some progress on closing the diagnostic gap between women and men. As stated previously, the common guidelines prefer hs-cTn assays above the conventional cTn assays, which have been introduced in Europe around 2012. ${ }^{2} \mathrm{Hs}$-cTn assays should meet two analytical criteria: 1) the $99^{\text {th }}$ percentile upper reference limit of hs-cTn should not be lower than the $10 \%$ coefficient of variation (CV) cutoff, and 2) the proportion of individuals with measurable cTn concentrations (above the assay's limit of detection) should be $\geq 50 \%{ }^{37}$ Troponin assays that do not meet these criteria are defined as conventional cTn assays. Today there are two assays available which meet the high-sensitivity criteria: the hs-cTnT assay from Roche and Architect hs-cTnI assay from Abbott. ${ }^{37}$ Whereas clinical cutoff values of conventional cTn assays were limited to $10 \% \mathrm{CV}$, the technical improvement of hs-cTn assays has led that the clinical cutoff values of hs-cTn assays are lowered corresponding to the $99^{\text {th }}$ percentile upper reference limits from a reference population (hs-cTnT, $14 \mathrm{ng} / \mathrm{L}$; hs-cTnI, $26 \mathrm{ng} / \mathrm{L}$, package insert). This enabled that cTn concentrations could now be detected also in other high-risk CVD patients, as described above (Table 4.1). Somewhat misleading is that the higher prevalence of elevated cTn levels found by hs-cTn assays resulted 
into more "false-positive" results. Most of these unexpected hs-cTn elevations are "true positive" for (subclinical) myocardial injury (rather than AMI) and thus still require diagnostic workup..$^{38}$ Thus, the change from conventional cTn assays to hscTn assays, and thereby the lowering of the AMI cutoff values, has led to a higher proportion of individuals suitable for beneficial therapies. ${ }^{39}$

Thus far, no studies investigated the clinical impact of change over from conventional to hs-cTn assays for diagnosis of AMI in women and men separately. However, hypothetically, the change over from cTn to hs-cTn assays may have led that unrecognized AMIs in women who were missed in the past could now be detected with hs-cTn assays. At this moment the available evidence supporting this hypothesis is scarce and this topic is currently under investigation.

Another important aspect is that women have lower troponin levels as compared with men. ${ }^{10}$ A number of studies determined sex-specific $99^{\text {th }}$ percentile upper reference limits of hs-cTnT and hs-cTnI and showed that women-specific thresholds are remarkably lower as compared with men-specific thresholds..$^{9,10,40,41}$ As currently a universal diagnostic threshold of hs-cTn is recommended for diagnosing AMI, which is higher than women-specific thresholds, this may contribute to underdiagnosis of AMI in women. In addition, the recommended universal hs-cTnT and hs-cTnI thresholds (14 ng/L vs $26 \mathrm{ng} / \mathrm{L}$, respectively) are not biologically equivalent. ${ }^{2}$ A direct comparison of (sex-specific) $99^{\text {th }}$ percentile upper reference limits of hscTnT and hs-cTnI from a single reference population revealed numerically similar $99^{\text {th }}$ percentile upper reference limits (hs-cTnT, $15 \mathrm{ng} / \mathrm{L}$; hs-cTnI, $13 \mathrm{ng} / \mathrm{L}$ ) and enhanced further investigation into sex-specific analysis and downward adjustment of hs-cTnI threshold (Figure $4 \cdot 4$ ). ${ }^{40}$

Whether these sex inequalities are of such clinical relevance that the use of universal hs-cTn thresholds hampers the diagnosis of AMI in women, and sex-specific thresholds should be incorporated in diagnostic cTn algorithms, remains controversial. The consideration of sex-specific analysis should be carefully weighted, as this implementation also will result into more complexity of acute cardiac care management. Thus far, the limited number of studies who investigated this issue showed contradictory findings. ${ }^{43-48}$ Two studies recommended to remain the universal thresholds, as they did not observe differences in sex-specific diagnostic and prognostic performance of hscTnT on clinical outcome ${ }^{44}$, even when sex-specific thresholds were applied. ${ }^{43}$ Contrary to these findings, Schofer et al. found sex disparities in the diagnostic performance of AMI using hs-cTnI, particularly into the rule-in performance. ${ }^{45}$

The hypothesis that sex-specific thresholds reduce the underdiagnosis of AMI in women is reinforced by the findings of Shah et al., showing that the use of sex-specific hs-cTnI thresholds resulted into a doubling of AMI diagnosis in women but also that the prevalence of AMI diagnosis became similar for women and men..$^{4}$ They concluded that sex-specific clinical decision limits of hs-cTnI should be considered for further investigation to close the diagnostic gap between women and men. Two studies 
showed that applying sex-specific hs-cTnI thresholds did not lead to excessively falsepositive AMI diagnosis. 47,48

Altogether, this finding suggests that sex inequalities may be more relevant for the hs-cTnI assay than for the hs-cTnT assay. Large multicenter randomized controlled trials (e.g., clinical trials.gov NCT01852123) are currently ongoing that should clarify the clinical impact of incorporation of sex-specific cardiac troponin thresholds in the diagnostic algorithm of AMI.

\section{$>$ CARDIAC TROPONINS AS PROGNOSTIC BIOMARKER}

Despite conventional cTn assays were able to detect troponin levels in the clinical range seen in AMI patients, they were not suitable for further risk stratification or prognosis in lower-risk populations. With the shift to hs-cTn assays, cTn could also be detected in the general population, which makes cTn a new promising biomarker for risk stratification and prognosis of CVD morbidity and mortality in the general population. Thus far, a number of epidemiological studies have shown that basal hs-cTn levels are associated with cardiac morbidity and mortality in the general population..$^{31,49-51}$

Due to fact that there are pathophysiological differences between sexes resulting into different cTn levels in women and men, the clinical impact of sex on the associations between hs-cTn and CVD mortality is a topic of debate. ${ }^{52-55}$ While some studies showed no interaction of sex on the relationships with troponin and CVD mortality ${ }^{52,53}$, other studies did (Table 4.2).54,55 The inconsistency between these studies is probably a result of the divergence of age. Besides the sex effect, also age modifies the association between hs-cTn and mortality. In individuals $\geq 70$ years, the impact of sex seems not of clinical importance, as nonsignificant interaction terms of sex were found (all-cause mortality, $P_{\text {interaction }}=0.74$; incident CVD, $\left.P_{\text {interaction }}=0.71\right) .{ }^{22}$ However, in the lower age range, both for hs-cTnT and hs-cTnI higher prognostic values on CVD mortality and all-cause mortality were observed in women than in men, whereby the risk on mortality of both sexes increased in older individuals $\geq 65$ years..$^{54-57}$

The prognostic impact of sex-specific hs-cTn thresholds in the subgroup of patients with suspected ACS on CVD mortality is also not clarified yet. ${ }^{43,46,58,59}$ Shah et al. observed that both women and men susceptive of AMI that were reclassified as having AMI after using sex-specific thresholds had the highest risk on death and recurrent AMI after one year as compared with subjects without having AMI. ${ }^{46}$ In line with these results, Cullen et al. showed that particularly for women, sex-specific hs-cTnI thresholds improved the identification of women for CVD events. ${ }^{46,58}$ However, thus far, these observations are not confirmed for sexspecific hs-cTnT thresholds. ${ }^{43,59}$ Mueller-Hennessen stated that age might be the crucial factor that should be taken into account, instead of sex-specific analysis. ${ }^{59}$ Further study is needed to draw conclusions about this statement, and whether or not sex-specific thresholds of hs-cTnT and/or hs-cTnI improve the long-term prognosis in individuals with suspected ACS. 

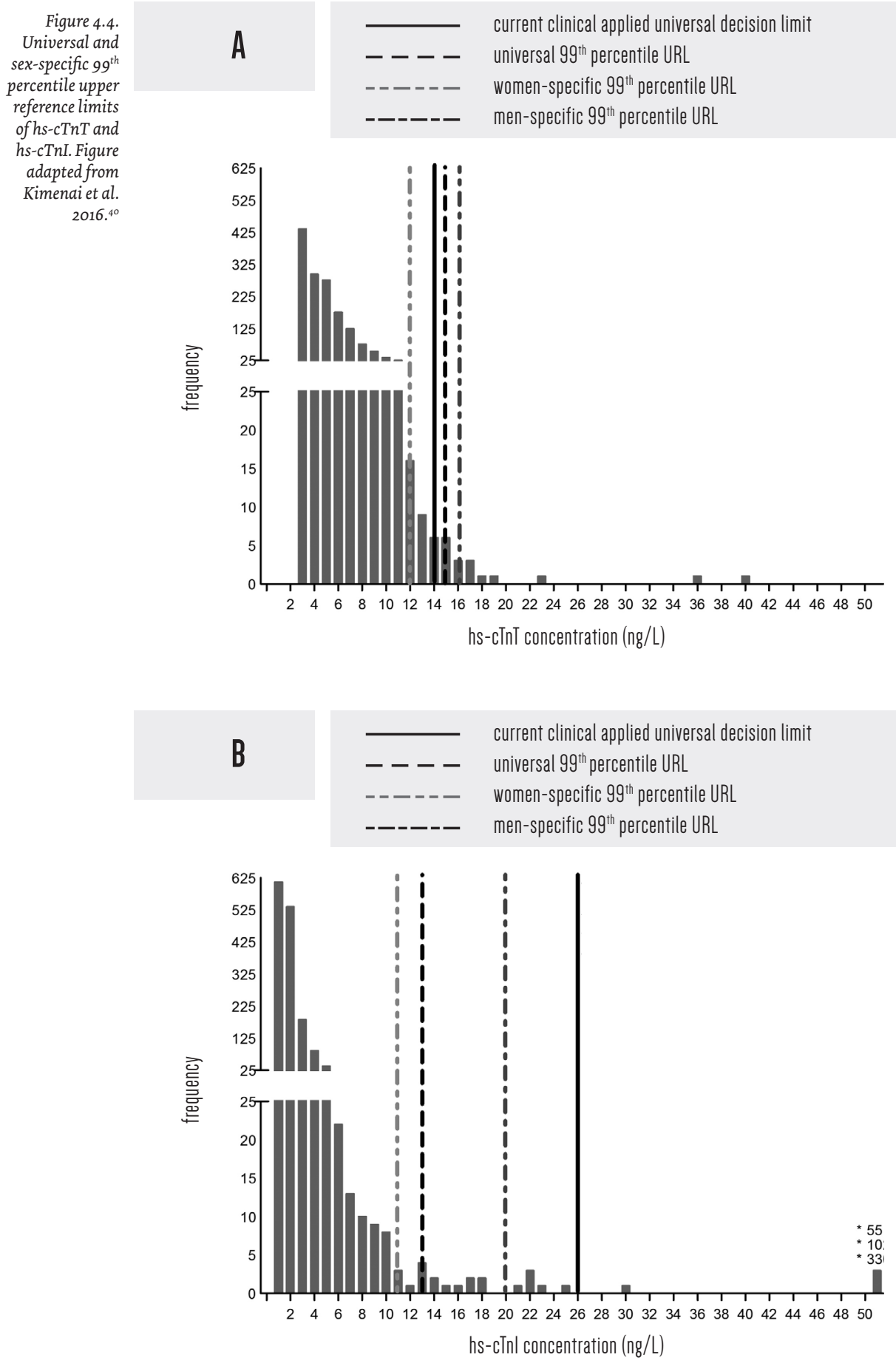


\begin{tabular}{|c|c|c|c|c|}
\hline Publication & Population & Median follow-up & Women/men & Outcome (HR, 95\% CI) \\
\hline $\begin{array}{l}\text { Zeller } \\
\text { et al. } 2014\end{array}$ & $\begin{array}{l}\text { Scottish Heart Health } \\
\text { Extended Cohort } \\
\text { (30- } 74 \text { years) }\end{array}$ & 20 years & $7742 / 7598$ & $\begin{array}{l}\text { CVD events } \\
\text { per cubic root hs-cInl increase } \\
\text { Women: I.35 (I.I8 - I.53) } \\
\text { Men: I.26 (I.I5 - I.38) } \\
\text { Coronary mortality } \\
\text { per cubic root hs-cInl increase } \\
\text { Women: I.53 (I.23 - I.92) } \\
\text { Men: I.35 (I.2I - I.50) } \\
\text { All-cause mortality } \\
\text { per In hs-cInT increase } \\
\text { Women: } 3.67 \text { (2.3I - 5.8I) } \\
\text { Men: } \quad 2.15 \text { (I.6I - } 2.87)\end{array}$ \\
\hline $\begin{array}{l}\text { Dallmeier } \\
\text { et al. } 2015\end{array}$ & $\begin{array}{l}\text { The ActiFE study } \\
\text { ( } \approx 65 \text { years) }\end{array}$ & 4.0 years & $618 / 804$ & $\begin{array}{l}\text { All-cause mortality } \\
\text { per In hs-cInl increase } \\
\text { Women: } \quad 3.33(2.15-5.18) \\
\text { Men: } \quad 1.92(1.55-2.38)\end{array}$ \\
\hline $\begin{array}{l}\text { Eggers } \\
\text { et al. } 2015\end{array}$ & $\begin{array}{l}\text { PIVUS study } \\
\text { (70 years) }\end{array}$ & 10 years & $502 / 502$ & $\begin{array}{l}\text { All-cause mortality } \\
\text { per In hs-cTnl increase } \\
\text { Women: } \quad 1.59(1.11-2.28) \\
\text { Men: } \quad 1.38(I .12-1.70) \\
\text { Cardiac morbidity: } \\
\text { incident CVD } \\
\text { per In hs-cTnl increase } \\
\text { Women: } \quad \text { I.23 (0.82 - I.86) } \\
\text { Men: } \quad \text { I.I4 (0.88 - I.47) }\end{array}$ \\
\hline $\begin{array}{l}\text { Omland } \\
\text { et al. } 2015\end{array}$ & $\begin{array}{l}\text { HUNT study } \\
(\geq 20 \text { years })\end{array}$ & 13.9 years & $5281 / 4431$ & $\begin{array}{l}\text { CVD mortality } \\
\text { per I-SD in In hs-cTnl increase } \\
\text { Women: I.44 (I.3I - I.58) } \\
\text { Men: } \quad \text { I.IO (I.00 - I.20) } \\
\text { All-cause mortality } \\
\text { per I-SD in In hs-cTnl increase } \\
\text { Women: I.33 (I.24 - I.42) } \\
\text { Men: } \quad \text { I.08 (I.0I - I.I5) }\end{array}$ \\
\hline
\end{tabular}

Table 4.2.

Characteristics and main findings of studies investigating clinical impact of sex on the relationship with hs-cTn and CVD morbidity and mortality in the general population. 


\section{NATRIURETIC PEPTIDES}

\section{$>$ THE BASICS OF NATRIURETIC PEPTIDES}

The heart muscle has an endocrine phenotype as it produces the cardiac hormones atrial natriuretic peptide (ANP) and B-type natriuretic peptide (BNP). ANP (28 amino acids (aa), Swiss-Prot Po116o) is primarily derived from cardiomyocytes from the atria, and BNP (32 aa, Swiss-Prot P1686o) is derived from cardiomyocytes from the ventricles, although it was first identified in porcine brain (brain-type). ANP and BNP are quite similar peptides that are released upon stimulation by stretching of the myocyte, as often seen with an overfilled heart, thereby causing excretion of sodium and water. Besides promoting natriuresis and diuresis functionalities, their counter regulatory function also comprises vasodilation and inhibition of the renin-angiotensinaldosterone and sympathetic nervous system. The release of ANP is regulated by its release from storage granules into the circulation, while on the other hand the release of BNP is regulated on the level of its gene expression. C-type natriuretic peptide (CNP) (Swiss-Prot P23582) and D-type natriuretic peptide(DNP) are also part of the natriuretic peptide family but should not be considered as cardiac peptides in humans.

The bioactive hormonal end products ANP and BNP are derived from their precursors, first in the form of preprohormones and next as prohormones. Human proBNP (108 aa) is cleaved at position $73-76$, leading to the inactive side product $\mathrm{N}$-terminal fragment proBNP 1-76 (NT-proBNP) and the active $\mathrm{C}$-terminal fragment proBNP 77-108 being known as BNP or BNP-32. Intact proBNP has an expected mass of $11 \mathrm{kDa}$, but in the mid-region (proBNP 36-71) glycosylation occurs to a fully or partially extent leading to unclarified masses that are estimated to be around $25 \mathrm{kDa}$. For the bioactivity of BNP is the ring structure essential, formed by a disulfide bond, as is synthesized in the endoplasmic reticulum and as is necessary for receptor binding and biological activity.

Natriuretic peptide concentrations in the blood circulation are fully integrated into clinical practice as important diagnostic and prognostic biomarkers. A number of different commercial immunoassays are available, especially for BNP and NTproBNP; for NT-proBNP there is just one source of antibodies and calibrators (Roche Diagnostics). In 2005 the IFCC initiated recommendations on analytical and preanalytical quality specifications and to improve assay harmonization. ${ }^{60-62} \mathrm{BNP}$ is especially susceptible to degradation that may affect antibody affinity. Only EDTAanticoagulated plastic tubes are acceptable and samples should be immediately put on ice and measured as soon as possible, since BNP is not stable at room temperature and at $2-8^{\circ} \mathrm{C}$ it is stable for just a few hours. ${ }^{63}$ For NT-proBNP the main issue is the potential of cross-reactivity with split products of NT-proBNP and proBNP. ${ }^{64}$ The extent of glycosylation does not play a role since antibodies are selected outside the region with glycosylation sites. NT-proBNP is measurable in serum or plasma $(10 \%$ lower in EDTA plasma) and is stable for at least $48 \mathrm{~h}$ at room temperature. ${ }^{63}$ 
Upon stretching of cardiomyocytes, proBNP is split into equimolar amounts of BNP and NT-proBNP, and indeed, they are used for similar clinical purposes as is pointed out further in this chapter. ${ }^{3,4}$ However, their concentrations in the blood circulation are absolutely different and values are not interchangeable, also for other reasons than the previously discussed pre-analytics and stability. Namely, BNP and NT-proBNP are both cleared by the kidneys, but BNP is besides that also cleared by natriuretic peptide receptor type $C$ (NPR-C) and neural endopeptidases. This results in a half-life for BNP of approximately 25 minutes and for NT-proBNP this is estimated to be twice as long with approximately 120 minutes. Finally, both peptides have different molecular weights, with the conversion factor for BNP from $\mathrm{pmol} / \mathrm{L}$ to $\mathrm{pg} / \mathrm{mL}$ being 3.460 and for NT-proBNP 8.457.

\section{$>$ NATRIURETIC PEPTIDES IN HEALTHY WOMEN AND MEN}

Early studies already illustrated significantly higher natriuretic peptide concentrations in women than in men ${ }^{11,12,65}$ suggesting a close relation between the cardiac endocrine function and the sex steroid hormones. Figure 4.5 illustrates a typical NT-proBNP distribution as found in our reference population. ${ }^{10}$ The sex effect remained true even after correction for differences in body composition and LV mass. ${ }^{66}$

Interestingly, sex-based differences in natriuretic peptide concentrations seem to be more pronounced for premenopausal women than for postmenopausal women. It was observed that BNP and NT-proBNP concentrations were lower in postmenopausal women as compared to premenopausal women ${ }^{66,67}$ though this difference could not be confirmed by others. ${ }^{68}$ Also, BNP and NT-proBNP concentrations in postmenopausal women seem to become closer to the concentrations as found in men, as was especially true for older adults of 70-75 years and older ${ }^{55,69}$, while others reported higher NT-proBNP concentrations for women independent of their hormonal and menopausal status. ${ }^{65}$ Table 4.3 illustrates that we also found higher NT-proBNP concentrations for women when compared to men, irrespective of their age, with the lowest NT-proBNP concentrations for women who almost reached or just reached their menopause ( 40 - 50 years). The latter was found by some ${ }^{65}$ but not by all ${ }^{55,69}$ as discussed in more detail below in this chapter.

It has been hypothesized that the sex effect on natriuretic peptides is caused by a stimulatory effect of estrogens and an inhibitory effect of androgens, as schematically illustrated in Figure 4.6. NT-proBNP levels indeed were inversely associated with androgen concentrations (total and free testosterone ${ }^{67,68}$, though the association with estradiol was found to be weak or not significant at all. ${ }^{68,70}$ The latter is not completely surprising because of the following issues. The biological variation in estradiol concentrations is great, up to fivefold during a menstrual cycle. Strikingly, NT-proBNP concentrations were lower in the mid cycle phase than in the follicular or luteal phase and thus completely opposite to the fluctuations of estradiol. ${ }^{67}$ Moreover, estradiol ranges in cycling women are $\geq 2$ times 

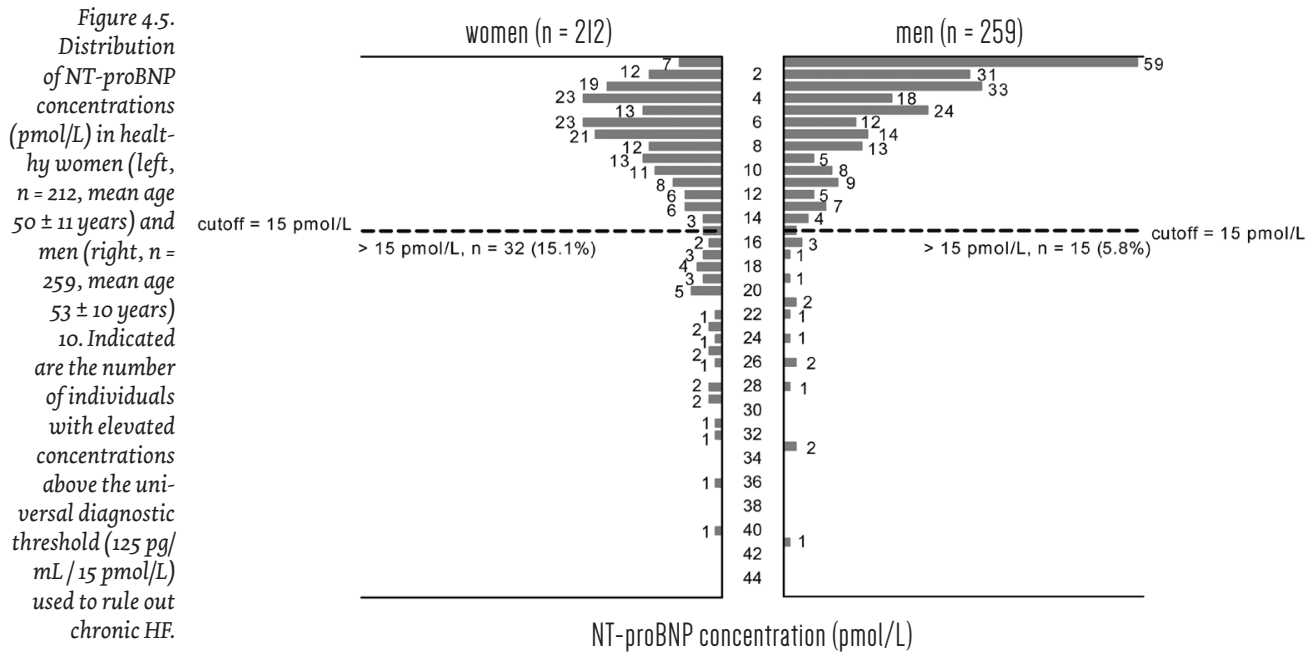

greater than in men, while testosterone ranges in women are hardly to mention in comparison to the concentrations found in men (total testosterone, factor 10; free testosterone, factor 30 ). There might also be an alternative explanation via the indirect role of sex hormone-binding globulin (SHBG) which synthesis is stimulated by estrogens and inhibited by androgens ${ }^{71}$ and SHBG was indeed positively associated with BNP and NT-proBNP concentrations. ${ }^{68}$ Anyway, the explanation for this sex hormone phenomenon is not understood yet. The current hypothesis is 
that fertile women benefit from the cardioprotective actions of natriuretic peptides, including vasodilation and diuresis/natriuresis, as is also noticed by the lower risk for cardiovascular events for women during their cycling. ${ }^{5}$

Clinical studies demonstrate that also interventions that affect sex hormones in women and men fit with previous observations. For instance, BNP and NT-proBNP concentrations were higher in premenopausal women who receive estrogens for contraception $^{67}$ or postmenopausal women who receive estrogens for hormone replacement therapy ${ }^{72}$, as compared to premenopausal women. Moreover, androgen receptor blockage and, to a lesser extent, androgen suppression in men with prostate cancer also result in increased NT-proBNP concentrations. ${ }^{73}$

\section{$>$ NATRIURETIC PEPTIDES DURING PREGNANCY}

Of special clinical interest is the relation of natriuretic peptides with pregnancy. Normal pregnancy goes along with median concentrations about twice that of nonpregnant controls, rising early in pregnancy and remaining high throughout gestation until $\approx 72$ hours after delivery. ${ }^{74}$ Complications including acute HF may be triggered by eclampsia or preeclampsia and in such situations NT-proBNP might possibly help in the diagnosis ${ }^{75}$ though this is not (yet) widely established in routine clinical care. ${ }^{7}$ It has been proven though that in preeclampsia NT-proBNP may reflect ventricular stress and subclinical cardiac dysfunction worsening if fetal growth restriction is present. ${ }^{77}$

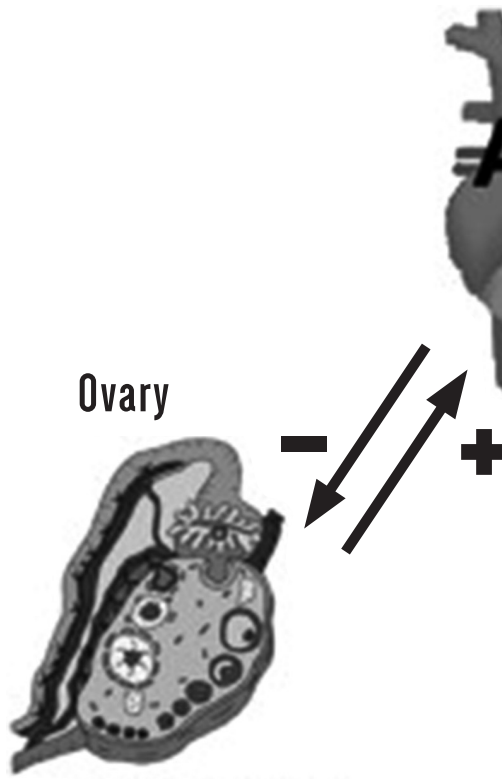

Estrogens

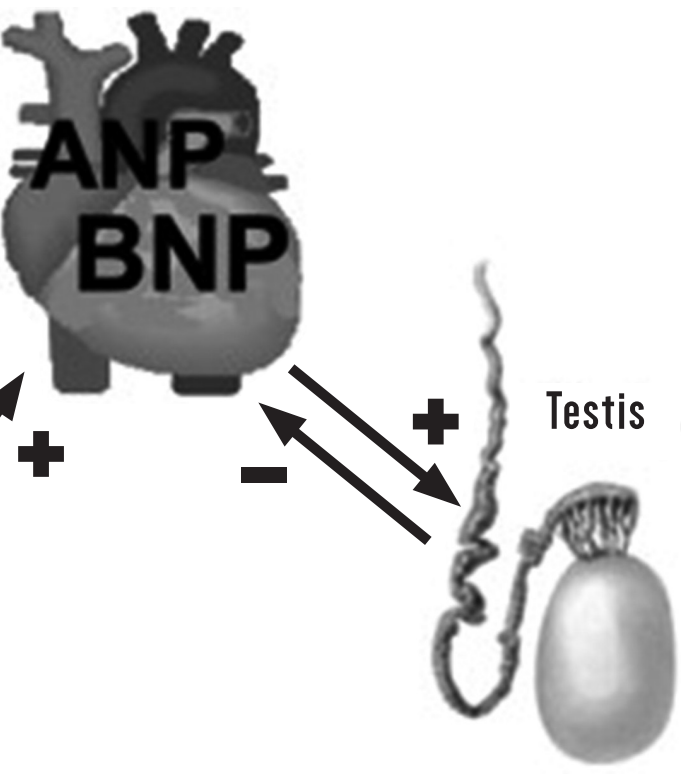

Androgens
Figure 4.6.

Schematic

representation of the relationship between cardiac endocrine and gonadal functions in women and men, showing a stimulatory effect of estrogens (arrow) and an inhibitory effect of androgens (blockade). Figure adapted from Maffei et al. $2001 .^{71}$ 


\section{$>$ NATRIURETIC PEPTIDES AND AGE}

Also the influence of age on baseline natriuretic peptide concentrations is very strong ${ }^{61}$ and might even more be important in clinical patients than in healthy individuals. ${ }^{65}$ Table 4.3 shows that we indeed found substantially higher NT-proBNP concentrations for older adults. Moreover, as mentioned before, NT-proBNP concentrations in our population were higher for women than for men and this was true for all age categories $(\mathrm{P}<0.05)$, similar to what was found by some $\mathrm{e}^{65}$, while others found equal NT-proBNP concentrations for postmenopausal women and men.55,69 When focusing on the age effect, it is immediately clear that many of the older healthy individuals exceed the diagnostic cutoff that is used to rule out (chronic) HF. This is especially the case for adults with an age of 70-75 years and older, as also illustrated by Figure 4.7. It is therefore crucial to remind that the current cutoffs are designed for ruling out HF but that other diagnostic tools are necessary for further diagnostic workup in the ruling in. ${ }^{3,4}$

Other clinical factors that influence natriuretic peptide concentrations are body mass index or obesity, ethnicity and non-HF pathologies ${ }^{3,4,78}$ and are not described here in great detail. Explicit attention should be given to renal impairment which substantially increases NT-proBNP concentrations and to a lesser extent also BNP concentrations. ${ }^{79,80}$

\footnotetext{
Figure 4.7

Age- and sex-

specific $80^{\text {th }}$

percentile upper

reference limits

for NT-proBNP

concentrations

in healthy

individuals

and stage $A / B$

HF subjects.

The universal diagnostic

threshold to rule

out chronic HF

is set at $125 \mathrm{pg} /$ $\mathrm{mL}$ or $15 \mathrm{pmol} / \mathrm{L}$

Adapted from McKie et al. $2010^{69}$
}

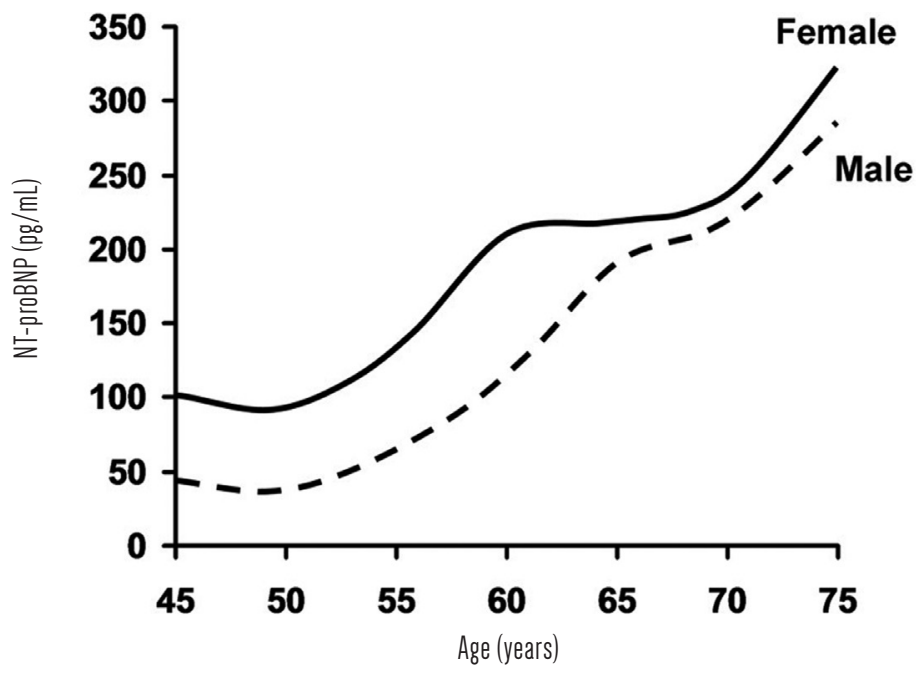

\section{$>$ NATRIURETIC PEPTIDES FOR THE DIAGNOSIS OF HEART FAILURE}

The definition of HF is mainly based on the measurement of the left ventricular ejection fraction (LVEF) and includes a wide range of patients, from those with normal to reduced LVEF. Table 4.4 shows that natriuretic peptides are also part of the criteria for HF, and with the recent ESC and ACC/AHA guideline updates ${ }^{3,4}$ their role became even more important for diagnostic purposes, establishing disease severity, and prognosis. 


\begin{tabular}{llll}
\hline Criteria & HFrEF & HFmrEF & HFpEF \\
\hline I & Symptoms \pm signs & $\begin{array}{l}\text { Symptoms } \pm \text { signs } \\
\text { LVEF }<40 \%\end{array}$ & Symptoms \pm signs \\
3 & $\begin{array}{l}\text { I. Elevated levels of } 40-49 \% \\
\text { natriuretic peptides }\end{array}$ & $\begin{array}{l}\text { LVEF } \pm 50 \% \\
\text { I. Elevated levels of } \\
\text { natriuretic peptides }\end{array}$ \\
& $\begin{array}{l}\text { 2. At least one } \\
\text { additional criterion }\end{array}$ & $\begin{array}{l}\text { 2. At least one } \\
\text { additional criterion }\end{array}$ \\
\hline
\end{tabular}

In the diagnostic workup, two algorithms are prescribed as summarized in the following part. ${ }^{3}$ They use either BNP or NT-proBNP, though absolute values and diagnostic thresholds are not interchangeable 4 , and to a lesser extent also midregional proANP (MR-proANP). There are also several analytical and clinical aspects like sex and age that should be considered when using natriuretic peptides for clinical use. ${ }^{61}$

In the non-acute setting ${ }^{3}$, the diagnosis of HF is assessed by clinical history, physical examination, and ECG. If all elements are normal, HF is highly unlikely and other diagnoses need to be considered. If at least one element is abnormal, natriuretic peptides should be measured, if available, to identify those who need echocardiography. The upper limit of normal in the non-acute setting for BNP is 35 $\mathrm{pg} / \mathrm{mL}$ (10 pmol/L), and for NT-proBNP, it is $125 \mathrm{pg} / \mathrm{mL}(15 \mathrm{pmol} / \mathrm{L})$.

In the acute setting ${ }^{3}$, the diagnostic workup is somewhat different. Upon presentation with acute dyspnea and the suspicion of acute HF, several diagnostic tests are recommended to differentiate cardiac causes of acute dyspnea from noncardiac causes. Natriuretic peptides should be assessed in all patients, and here higher thresholds should be used, namely, for BNP $100 \mathrm{pg} / \mathrm{mL}$ ( $35 \mathrm{pmol} / \mathrm{L})$, and for NT-proBNP $300 \mathrm{pg} / \mathrm{mL}$ (35 pmol/L) and/or MR-proANP $120 \mathrm{pmol} / \mathrm{L}$. Other diagnostic tests that are recommended include ECG; X-thorax; other laboratory assessments like cTn, urea, creatinine, electrolytes (sodium, potassium), glucose, complete blood count, liver function tests, and thyroid stimulating hormone (TSH); and finally, echocardiography.

The use of natriuretic peptides is especially recommended for ruling out HF in patients who present with dyspnea, since their negative predictive values are very high (0.94 - 1.00), both in the non-acute and the acute settings. ${ }^{3,81,82}$ This remains true even when using a universal diagnostic threshold that are regardless of age $\mathrm{e}^{{ }_{1}}$ or $\operatorname{sex}^{82}$ or any of the other risk factors. It was first thought to use age-dependent cutoffs (US Food and Drug Administration, FDA-cleared cutoffs), $125 \mathrm{pg} / \mathrm{mL}$ (15 pmol/L) 
for patients younger than 75 years and $450 \mathrm{pg} / \mathrm{mL}(50 \mathrm{pmol} / \mathrm{L})$ for patients of 75 years and older, and/or renal-dependent cutoffs for eGFR $<60 \mathrm{ml} / \mathrm{min} / 1.72 \mathrm{~m}^{2} .^{79,80}$ However, clinical research has inevitably shown that negative predictive values were hardly affected when applying a single and relatively low cutoff that is independent from risk factors and thus easier to use in daily practice and still safe for all types of patients.

In contrast, natriuretic peptides are less appropriate for establishing the diagnosis of $\mathrm{HF}$ with positive predictive values in the non-acute setting of only $0.44-0.57$ and in the acute setting of $0.66-0.67 .^{3}$ This could be mainly explained by the numerous cardiovascular and non-cardiovascular causes of elevated natriuretic peptides, as summarized in Table $4 \cdot 5^{3,4,78}$ with atrial fibrillation, age, and renal failure being important if not the most important factors. ${ }^{3}$

Table 4.5. Causes of elevated

HF, including RV syndromes

Acute coronary syndromes

Heart muscle disease, including LVH

Valvular heart disease

Pericardial disease

Atrial fibrillation

Myocarditis

Cardiac surgery

Cardioversion

Toxic-metabolic myocardial insults, including cancer chemotherapy

\section{Noncardiac}

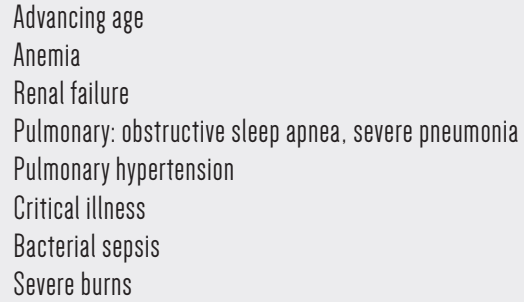

The severity of CHF is significantly associated with natriuretic peptide concentrations as, for instance, determined by the New York Heart Association classification. ${ }^{81,83}$ Concentrations are lower for HF patients with preserved ejection fraction (HFpEF) than for patients with reduced ejection fraction $(\mathrm{HFrEF})^{84}$, but diagnostic values apply similarly to both groups. ${ }^{3}$ 


\section{$>$ NATRIURETIC PEPTIDES FOR THE MANAGEMENT OF HEART FAILURE}

Over the last 15 years, it has been hypothesized that natriuretic peptides might be helpful as objective measures in the management of HF patients. Most of the prospective clinical trials investigated whether a fixed target concentration of natriuretic peptides is useful to titrate the pharmacologic therapy to. Results of single studies have been quite conflicting, unfortunately, whereas meta-analyses overall show promising effects. The most recent and largest meta-analysis by Savarese et al. included 2686 patients from 12 studies. ${ }^{85}$ They reported benefits on both hospitalization and mortality of the HF patients. Significant results were obtained specifically for NT-proBNP-guided studies, in contrast to the BNP-guidedstudies. The final evidence was expected from the GUIDE-IT (Guiding Evidence Based Therapy Using Biomarker Intensified Treatment in Heart Failure) study by investigating high-risk patients with relatively advanced $\mathrm{HFrEF}^{86}$, but NT-proBNP guided therapy turned out not to be more effective in hospitalization or mortality than usual care strategy nor were doses of medical therapy different between the groups; this study was therefore stopped for futility when 894 of the 1100 patients were enrolled. ${ }^{87}$

Sex-based differences for natriuretic peptide-guided therapy have not been reported, though this is different for the influence of age. The benefits for NT-proBNP guidance appear at least to be true for individuals of 75 years or younger ${ }^{84,88,89}$ and for patients with HFrEF but not for HFpEF. ${ }^{84}$ It is conceivable that the more frequent presence of comorbidities in elderly may prevent or even promote potentially harmful uptitration of drugs.

Unfortunately, the concept of serial sampling of natriuretic peptides is quite limited because of a great biological variation which is of course crucial for successful HF management. Changes in concentration of more than 50 to $100 \%$ are necessary to be sure that the changes observed are "real" (exceeding reference change values) and are indeed due to therapy interventions..$^{90,91}$ It was recently validated and confirmed that the biological variation is independent from the clinical condition of the patient, thus being equal in healthy individuals and chronic HF patients. ${ }^{92}$

\section{$>$ NATRIURETIC PEPTIDES FOR PREVENTION, PROGNOSIS, AND RISK STRATIFICATION}

Numerous prognostic markers of death and hospitalization have been identified in HF patients. Extensive review and meta-analysis illustrates that especially age and to a lesser extent also sex are strong predictors for cardiac death and hospitalization in patients with HF, if not the most important ones. ${ }^{93,94}$ In the top ten of the most prominent predictors is also space for a couple of biomarkers that are related to renal function (sodium, creatinine, urea), oxygen supply (hemoglobin), and cardiac function (NT-proBNP). ${ }^{93}$

The use of natriuretic peptides as prognostic predictors remains however challenging for daily practice. ${ }^{3}$ Nevertheless, the recent American guideline 
included new level 1 recommendations on the use of natriuretic peptides as powerful predictors with a specific role for 1) screening in patients at risk for developing HF (presence of hypertension, diabetes mellitus, or vascular disease), 2) baseline levels on admission to the hospital in case of acutely decompensated HF, 3) a predischarge level during hospitalization, and 4) in case of chronic HF to consider also biomarkers of myocardial injury (cTn) or fibrosis. ${ }^{4}$ It seems almost logical that sex and probably also age are very important predictors that should always be considered when using these applications, but future evidence has to proof this first.

An interesting sex aspect is the benefit that fertile women gather from their higher baseline natriuretic peptide concentrations, as previously discussed. ${ }^{5}$ The survival advantage of women though was canceled out in case of congenital heart disease what might be related to the higher prevalence of severe pulmonary hypertension. ${ }^{95}$ Also, despite that, marked sex differences were found in acute HF patients with preserved versus reduced ejection fractions with opposite associations of anemia and LVEF requiring further attention. ${ }^{96}$ Even in community-dwelling older adults sex-based differences remain an ongoing research topic where associations of NTproBNP with all-cause mortality were substantially stronger among women. ${ }^{55}$

\section{CONCLUSIONS AND FUTURE PERSPECTIVES}

Taken together, the cardiac biomarkers cTn, NT-proBNP, and BNP are the cornerstones in the diagnosis and clinical management of AMI and HF, respectively. Current cardiac care guidelines do not make a distinction between women and men and make use of "one size fits all" algorithms.

The debate on sex-specific analysis mainly concerns the underdiagnosis of AMI in women. Due to the improvement of the cTn assays, a new era arose where cTn levels became measurable in apparently healthy individuals, whereby lower cTn levels (and $99^{\text {th }}$ percentile upper reference limits) were observed in women as compared with men. The evidence for sex-specific diagnostic thresholds remains though controversial, and ongoing and future trials will clarify whether or not sex-specific analysis should be incorporated in the diagnostic algorithm of AMI.

Also other aspects of cTn algorithms are under investigation that may improve the diagnosis of AMI. For instance, combined biomarker approaches are investigated, as promising new biomarkers such as copeptin, heart fatty acid-binding protein, ST2, and growth differentiation factor-15 may have additive diagnostic value over hs-cTn. Up till now, however, the results are quite controversial, probably due to the already high diagnostic performance of hs-cTn. Furthermore, the hs-cTn assays allow further investigation to rapid measurement strategies, which could lead to faster diagnosis of AMI and, eventually, resulting into better prognosis after AMI. 
For the natriuretic peptides, previous studies observed higher natriuretic peptide concentrations in women, especially in premenopausal women, than in men. Natriuretic peptides are particularly incorporated in the ruling-out algorithms for the diagnosis of HF and, in contrast, to hs-cTn, where clinical research has shown that negative predictive values for ruling out HF were hardly affected when applying a universal diagnostic threshold of natriuretic peptides that is independent from sex or other risk factors.

Finally, we believe that in the future most sex-specific benefits of cardiac biomarkers can be obtained in the field of patient follow-up and in the field of cardiac risk prevention and risk stratification. Both are unfinished areas and fit the modern ideas of preventive and personalized medicine: from guiding therapy and prognostic applications for patients who are already diagnosed to patients at risk and in the future possibly to even healthy individuals. 
REFERENCES

1. Roffi M, Patrono C, Collet JP, et al. 2015 ESC Guidelines for the management of acute coronary syndromes in patients presenting without persistent ST-segment elevation: Task Force for the Management of Acute Coronary Syndromes in Patients Presenting without Persistent ST-Segment Elevation of the European Society of Cardiology (ESC). European heart journal 2016;37(3):267-315.

2. Thygesen $\mathrm{K}$, Alpert JS, Jaffe AS, et al. Third universal definition of myocardial infarction. European heart journal 2012;33(20):2551-67.

3. Ponikowski P, Voors AA, Anker SD, et al. 2016 ESC Guidelines for the diagnosis and treatment of acute and chronic heart failure: The Task Force for the diagnosis and treatment of acute and chronic heart failure of the European Society of Cardiology (ESC)Developed with the special contribution of the Heart Failure Association (HFA) of the ESC. Eur Heart J 2016;37(27):2129-200.

4. Yancy CW, Jessup M, Bozkurt B, et al. 2017 ACC/AHA/HFSA Focused Update of the 2013 ACCF/ AHA Guideline for the Management of Heart Failure: A Report of the American College of Cardiology/American Heart Association Task Force on Clinical Practice Guidelines and the Heart Failure Society of America. J Am Coll Cardiol 2017.

5. Mozaffarian D, Benjamin EJ, Go AS, et al. Heart Disease and Stroke Statistics-2016 Update: A Report From the American Heart Association. Circulation 2016;133(4):e38-36o.

6. Pagidipati NJ, Peterson ED. Acute coronary syndromes in women and men. Nat Rev Cardiol 2016;13(8):471-80.

7. Daniels LB, Maisel AS. Cardiovascular biomarkers and sex: the case for women. Nat Rev Cardiol 2015;12(10):588-96.

8. Alpert JS, Thygesen K, Antman E, et al. Myocardial infarction redefined--a consensus document of The Joint European Society of Cardiology/American College of Cardiology Committee for the redefinition of myocardial infarction. J Am Coll Cardiol 2000;36(3):959-69.

9. Apple FS, Ler R, Murakami MM. Determination of 19 cardiac troponin I and T assay $99^{\text {th }}$ percentile values from a common presumably healthy population. Clinical chemistry 2012;58(11):1574-81.

10. Mingels A, Jacobs L, Michielsen E, et al. Reference population and marathon runner sera assessed by highly sensitive cardiac troponin $\mathrm{T}$ and commercial cardiac troponin $\mathrm{T}$ and $\mathrm{I}$ assays. Clinical chemistry 2009;55(1):101-8.

11. Raymond I, Groenning BA, Hildebrandt PR, et al. The influence of age, sex and other variables on the plasma level of $\mathrm{N}$-terminal pro brain natriuretic peptide in a large sample of the general population. Heart 2003;89(7):745-51.

12. Redfield MM, Rodeheffer RJ, Jacobsen SJ, et al. Plasma brain natriuretic peptide concentration: impact of age and gender. J Am Coll Cardiol 2002;40(5):976-82. 
13. Apple FS, Collinson PO, Biomarkers ITFoCAoC. Analytical characteristics of highsensitivity cardiac troponin assays. Clin Chem 2012;58(1):54-61.

14. deFilippi C, Seliger SL, Kelley W, et al. Interpreting cardiac troponin results from high-sensitivity assays in chronic kidney disease without acute coronary syndrome. Clin Chem 2012;58(9):1342-51.

15. Cardinaels EP, Mingels AM, van Rooij T, et al. Time-dependent degradation pattern of cardiac troponin $\mathrm{T}$ following myocardial infarction. Clinical chemistry 2013;59(7):1083-90.

16. Labugger R, Organ L, Collier C, et al. Extensive troponin I and T modification detected in serum from patients with acute myocardial infarction. Circulation 2000;102(11):1221-6.

17. Jacobs EJH, Mingels AMA, Dieijen van - Visser MP. Cardiac Biomarkers in End-Stage Renal Disease. In: Sahay M, ed. Chronic Kidney Disease and Renal Transplantation: InTech Publication 2012:147-6o.

18. Freda BJ, Tang WH, Van Lente F, et al. Cardiac troponins in renal insufficiency: review and clinical implications. J Am Coll Cardiol 2002;40(12):2065-71.

19. Abbas NA, John RI, Webb MC, et al. Cardiac troponins and renal function in nondialysis patients with chronic kidney disease. Clin Chem 2005;51(11):2059-66.

20. Buiten MS, de Bie MK, Rotmans JI, et al. Serum Cardiac Troponin-I is Superior to Troponin-T as a Marker for Left Ventricular Dysfunction in Clinically Stable Patients with End-Stage Renal Disease. PLoS One 2015;10(8):e0134245.

21. Wallace TW, Abdullah SM, Drazner MH, et al. Prevalence and determinants of troponin T elevation in the general population. Circulation 2006;113(16):1958-65.

22. Scheven L, de Jong PE, Hillege HL, et al. High-sensitive troponin T and $\mathrm{N}$-terminal pro-B type natriuretic peptide are associated with cardiovascular events despite the cross-sectional association with albuminuria and glomerular filtration rate. Eur Heart J 2012;33(18):2272-81.

23. Martens RJ, Kimenai DM, Kooman JP, et al. Estimated Glomerular Filtration Rate and Albuminuria Are Associated with Biomarkers of Cardiac Injury in a Population-Based Cohort Study: The Maastricht Study. Clin Chem 2017;63(4):887-97.

24. Kimenai DM, Martens RJH, Kooman JP, et al. Troponin I and $\mathrm{T}$ in relation to cardiac injury detected with electrocardiography in a population-based cohort - The Maastricht Study. Sci Rep 2017;7(1):6610.

25. Eggers KM, Lindahl B. Impact of Sex on Cardiac Troponin Concentrations-A Critical Appraisal. Clinical chemistry 2017;63(9):1457-64.

26. Jaffe AS, Apple FS. High-sensitivity cardiac troponin assays: isn't it time for equality? Clin Chem 2014;60(1):7-9.

27. Westerman $S$, Wenger NK. Women and heart disease, the underrecognized burden: sex differences, biases, and unmet clinical and research challenges. Clin Sci (Lond) 2016;130(8):551-63.

28. Donaldson C, Eder S, Baker C, et al. Estrogen attenuates left ventricular and cardiomyocyte hypertrophy by an estrogen receptor-dependent pathway that increases calcineurin degradation. Circ Res 2009;104(2):265-75, 11p following 75 .

29. Piro M, Della Bona R, Abbate A, et al. Sex-related differences in myocardial remodeling. J Am Coll Cardiol 2010;55(11):1057-65.

30. de Simone G, Devereux RB, Daniels SR, et al. Gender differences in left ventricular growth. Hypertension 1995;26(6 Pt 1):979-83.

31. de Lemos JA, Drazner MH, Omland T, et al. Association of troponin T detected with a highly sensitive assay and cardiac structure and mortality risk in the general population. JAMA 2010;304(22):2503-12. 
32. Neeland IJ, Drazner MH, Berry JD, et al. Biomarkers of chronic cardiac injury and hemodynamic stress identify a malignant phenotype of left ventricular hypertrophy in the general population. Journal of the American College of Cardiology 2013;61(2):187-95.

33. Khan NA, Daskalopoulou SS, Karp I, et al. Sex differences in acute coronary syndrome symptom presentation in young patients. JAMA Intern Med 2013;173(20):1863-71.

34. Canto JG, Goldberg RJ, Hand MM, et al. Symptom presentation of women with acute coronary syndromes: myth vs reality. Arch Intern Med 2007;167(22):2405-13.

35. Elsaesser A, Hamm CW. Acute coronary syndrome: the risk of being female. Circulation 2004;109(5):565-7.

36. de Torbal A, Boersma E, Kors JA, et al. Incidence of recognized and unrecognized myocardial infarction in men and women aged 55 and older: the Rotterdam Study. European heart journal 2006;27(6):729-36.

37. Apple FS, Sandoval Y, Jaffe AS, et al. Cardiac Troponin Assays: Guide to Understanding Analytical Characteristics and Their Impact on Clinical Care. Clinical chemistry 2017;63(1):73-81.

38. Twerenbold R, Boeddinghaus J, Nestelberger T, et al. Clinical Use of High-Sensitivity Cardiac Troponin in Patients With Suspected Myocardial Infarction. Journal of the American College of Cardiology 2017;70(8):996-1012.

39. Eggers KM, Lindahl B, Melki D, et al. Consequences of implementing a cardiac troponin assay with improved sensitivity at Swedish coronary care units: an analysis from the SWEDEHEART registry. European heart journal 2016;37(30):2417-24.

40. Kimenai DM, Henry RM, van der Kallen CJ, et al. Direct comparison of clinical decision limits for cardiac troponin T and I. Heart 2016;102(8):610-6.

41. Gore MO, Seliger SL, Defilippi CR, et al. Age- and sex-dependent upper reference limits for the high-sensitivity cardiac troponin T assay. Journal of the American College of Cardiology 2014;63(14):1441-8.

42. Wildi K, Gimenez MR, Twerenbold R, et al. Misdiagnosis of Myocardial Infarction Related to Limitations of the Current Regulatory Approach to Define Clinical Decision Values for Cardiac Troponin. Circulation 2015;131(23):2032-40.

43. Rubini Gimenez M, Twerenbold R, Boeddinghaus J, et al. Clinical Effect of Sex-Specific Cutoff Values of High-Sensitivity Cardiac Troponin T in Suspected Myocardial Infarction. JAMA Cardiol 2016;1(8):912-20.

44. Balmelli C, Meune C, Twerenbold R, et al. Comparison of the performances of cardiac troponins, including sensitive assays, and copeptin in the diagnostic of acute myocardial infarction and long-term prognosis between women and men. Am Heart J 2013;166(1):30-7.

45. Schofer N, Brunner FJ, Schluter M, et al. Gender-specific diagnostic performance of a new high-sensitivity cardiac troponin I assay for detection of acute myocardial infarction. Eur Heart J Acute Cardiovasc Care 2017;6(1):60-68.

46. Shah AS, Griffiths M, Lee KK, et al. High sensitivity cardiac troponin and the underdiagnosis of myocardial infarction in women: prospective cohort study. BMJ 2015;350:g7873.

47. Sandoval Y, Smith SW, Schulz KM, et al. Diagnosis of type 1 and type 2 myocardial infarction using a high-sensitivity cardiac troponin I assay with sex-specific $99^{\text {th }}$ percentiles based on the third universal definition of myocardial infarction classification system. Clinical chemistry 2015;61(4):657-63. 
48. Trambas C, Pickering JW, Than M, et al. Impact of High-Sensitivity Troponin I Testing with Sex-Specific Cutoffs on the Diagnosis of Acute Myocardial Infarction. Clin Chem 2016;62(6):831-8.

49. Willeit P, Welsh P, Evans JDW, et al. High-Sensitivity Cardiac Troponin Concentration and Risk of First-Ever Cardiovascular Outcomes in 154,052 Participants. J Am Coll Cardiol 2017;70(5):558-68.

50. Blankenberg S, Salomaa V, Makarova N, et al. Troponin I and cardiovascular risk prediction in the general population: the BiomarCaRE consortium. Eur Heart J 2016;37(30):2428-37.

51. Sze J, Mooney J, Barzi F, et al. Cardiac Troponin and its Relationship to Cardiovascular Outcomes in Community Populations - A Systematic Review and Meta-analysis. Heart Lung Circ 2016;25(3):217-28.

52. Eggers KM, Johnston N, Lind L, et al. Cardiac troponin I levels in an elderly population from the community--The implications of sex. Clin Biochem 2015;48(12):751-6.

53. Saunders JT, Nambi V, de Lemos JA, et al. Cardiac troponin T measured by a highly sensitive assay predicts coronary heart disease, heart failure, and mortality in the Atherosclerosis Risk in Communities Study. Circulation 2011;123(13):1367-76.

54. Omland T, de Lemos JA, Holmen OL, et al. Impact of sex on the prognostic value of highsensitivity cardiac troponin I in the general population: the HUNT study. Clin Chem 2015;61(4):646-56.

55. Dallmeier D, Denkinger M, Peter R, et al. Sex-specific associations of established and emerging cardiac biomarkers with all-cause mortality in older adults: the ActiFE study. Clin Chem 2015;61(2):389-99.

56. Zeller T, Tunstall-Pedoe $\mathrm{H}$, Saarela $\mathrm{O}$, et al. High population prevalence of cardiac troponin I measured by a high-sensitivity assay and cardiovascular risk estimation: the MORGAM Biomarker Project Scottish Cohort. Eur Heart J 2014;35(5):271-81.

57. Lyngbakken MN, Rosjo H, Holmen OL, et al. Gender, High-Sensitivity Troponin I, and the Risk of Cardiovascular Events (from the Nord-Trondelag Health Study). Am J Cardiol 2016;118(6):816-21.

58. Cullen L, Greenslade JH, Carlton EW, et al. Sex-specific versus overall cut points for a high sensitivity troponin I assay in predicting 1-year outcomes in emergency patients presenting with chest pain. Heart 2016;102(2):120-6.

59. Mueller-Hennessen M, Lindahl B, Giannitsis E, et al. Diagnostic and prognostic implications using age- and gender-specific cut-offs for high-sensitivity cardiac troponin $\mathrm{T}$ - Subanalysis from the TRAPID-AMI study. Int J Cardiol 2016;209:26-33.

6o. Apple FS, Panteghini M, Ravkilde J, et al. Quality specifications for B-type natriuretic peptide assays. Clin Chem 2005;51(3):486-93.

61. Apple FS, Wu AH, Jaffe AS, et al. National Academy of Clinical Biochemistry and IFCC Committee for Standardization of Markers of Cardiac Damage Laboratory Medicine practice guidelines: Analytical issues for biomarkers of heart failure. Circulation 2007;116(5):e95-8.

62. Saenger AK, Rodriguez-Fraga O, Ler R, et al. Specificity of B-Type Natriuretic Peptide Assays: Cross-Reactivity with Different BNP, NT-proBNP, and proBNP Peptides. Clin Chem 2017;63(1):351-58.

63. Yeo KT, Wu AH, Apple FS, et al. Multicenter evaluation of the Roche NT-proBNP assay and comparison to the Biosite Triage BNP assay. Clin Chim Acta 2003;338(1-2):107-15. 
64. Luckenbill KN, Christenson RH, Jaffe AS, et al. Cross-reactivity of BNP, NT-proBNP, and proBNP in commercial BNP and NT-proBNP assays: preliminary observations from the IFCC Committee for Standardization of Markers of Cardiac Damage. Clin Chem 2008;54(3):619-21.

65. Costello-Boerrigter LC, Boerrigter G, Redfield MM, et al. Amino-terminal pro-B-type natriuretic peptide and B-type natriuretic peptide in the general community: determinants and detection of left ventricular dysfunction. J Am Coll Cardiol 2006;47(2):345-53.

66. Lew J, Sanghavi M, Ayers CR, et al. Sex-Based Differences in Cardiometabolic Biomarkers. Circulation 2017;135(6):544-55.

67. Lam CS, Cheng S, Choong K, et al. Influence of sex and hormone status on circulating natriuretic peptides. J Am Coll Cardiol 2011;58(6):618-26.

68. Chang AY, Abdullah SM, Jain T, et al. Associations among androgens, estrogens, and natriuretic peptides in young women: observations from the Dallas Heart Study. J Am Coll Cardiol 2007;49(1):109-16.

69. McKie PM, Cataliotti A, Lahr BD, et al. The prognostic value of N-terminal pro-B-type natriuretic peptide for death and cardiovascular events in healthy normal and stage $\mathrm{A} / \mathrm{B}$ heart failure subjects. J Am Coll Cardiol 2010;55(19):2140-7.

70. Saenger AK, Dalenberg DA, Bryant SC, et al. Pediatric brain natriuretic peptide concentrations vary with age and sex and appear to be modulated by testosterone. Clin Chem 2009;55(10):1869-75.

71. Clerico A, Fontana M, Vittorini S, et al. The search for a pathophysiological link between gender, cardiac endocrine function, body mass regulation and cardiac mortality: proposal for a working hypothesis. Clin Chim Acta 2009;405(1-2):1-7.

72. Maffei S, Del Ry S, Prontera $C$, et al. Increase in circulating levels of cardiac natriuretic peptides after hormone replacement therapy in postmenopausal women. Clin Sci(Lond) 2001;101(5):447-53.

73. Dockery F, Bulpitt CJ, Agarwal S, et al. Anti-androgens increase N-terminal pro-BNP levels in men with prostate cancer. Clin Endocrinol (Oxf) 2008;68(1):59-65.

74. Canobbio MM, Warnes CA, Aboulhosn J, et al. Management of Pregnancy in Patients With Complex Congenital Heart Disease: A Scientific Statement for Healthcare Professionals From the American Heart Association. Circulation 2017;135(8):e50-e87.

75. Tanous D, Siu SC, Mason J, et al. B-type natriuretic peptide in pregnant women with heart disease. J Am Coll Cardiol 2010;56(15):1247-53.

76. Gynaecologists RCoOa, Health NCCfWsaCs. Hypertension in Pregnancy: The Management of Hypertensive Disorders During Pregnancy. National Institute for Health and Clinical Excellence: Guidance 2010.

77. Giannubilo SR, Pasculli A, Tidu E, et al. Relationship between maternal hemodynamics and plasma natriuretic peptide concentrations during pregnancy complicated by preeclampsia and fetal growth restriction. J Perinatol 2017;37(5):484-87.

78. Group NW, Wu AH, Jaffe AS, et al. National Academy of Clinical Biochemistry laboratory medicine practice guidelines: use of cardiac troponin and B-type natriuretic peptide or N-terminal proB-type natriuretic peptide for etiologies other than acute coronary syndromes and heart failure. Clinical chemistry 2007;53(12):2086-96.

79. Anwaruddin S, Lloyd-Jones DM, Baggish A, et al. Renal function, congestive heart failure, and amino-terminal pro-brain natriuretic peptide measurement: results from the ProBNP Investigation of Dyspnea in the Emergency Department (PRIDE) Study. J Am Coll Cardiol 2006;47(1):91-7. 
8o. McCullough PA, Duc P, Omland T, et al. B-type natriuretic peptide and renal function in the diagnosis of heart failure: an analysis from the Breathing Not Properly Multinational Study. Am J Kidney Dis 2003;41(3):571-9.

81. Januzzi JL, Jr., Camargo CA, Anwaruddin S, et al. The N-terminal Pro-BNP investigation of dyspnea in the emergency department (PRIDE) study. Am J Cardiol 2005;95(8):948-54.

82. Krauser DG, Chen AA, Tung R, et al. Neither race nor gender influences the usefulness of amino-terminal pro-brain natriuretic peptide testing in dyspneic subjects: a ProBNP Investigation of Dyspnea in the Emergency Department (PRIDE) substudy. J Card Fail 2006;12(6):452-7.

83. Maisel AS, Krishnaswamy P, Nowak RM, et al. Rapid measurement of B-type natriuretic peptide in the emergency diagnosis of heart failure. N Engl J Med 2002;347(3):161-7.

84. Brunner-La Rocca HP, Eurlings L, Richards AM, et al. Which heart failure patients profit from natriuretic peptide guided therapy? A meta-analysis from individual patient data of randomized trials. Eur J Heart Fail 2015;17(12):1252-61.

85. Savarese G, Trimarco B, Dellegrottaglie S, et al. Natriuretic peptide-guided therapy in chronic heart failure: a meta-analysis of 2,686 patients in 12 randomized trials. PLoS One 2013;8(3):e58287.

86. Felker GM, Ahmad T, Anstrom KJ, et al. Rationale and design of the GUIDE-IT study: Guiding Evidence Based Therapy Using Biomarker Intensified Treatment in Heart Failure. JACC Heart Fail 2014;2(5):457-65.

87. Felker GM, Anstrom KJ, Adams KF, et al. Effect of Natriuretic Peptide-Guided Therapy on Hospitalization or Cardiovascular Mortality in High-Risk Patients With Heart Failure and Reduced Ejection Fraction: A Randomized Clinical Trial. JAMA 2017;318(8):713-20.

88. Pfisterer M, Buser P, Rickli $\mathrm{H}$, et al. BNP-guided vs symptom-guided heart failure therapy: the Trial of Intensified vs Standard Medical Therapy in Elderly Patients With Congestive Heart Failure (TIME-CHF) randomized trial. JAMA 2009;301(4):383-92.

89. Bayes-Genis A, Lupon J, Jaffe AS. Can Natriuretic Peptides be Used to Guide Therapy? EJIFCC 2016;27(3):208-16.

9o. $\mathrm{Wu} \mathrm{AH}$, Smith A. Biological variation of the natriuretic peptides and their role in monitoring patients with heart failure. Eur J Heart Fail 2004;6(3):355-8.

91. Wu AH, Smith A, Wieczorek S, et al. Biological variation for N-terminal pro- and B-type natriuretic peptides and implications for therapeutic monitoring of patients with congestive heart failure. Am J Cardiol 2003;92(5):628-31.

92. Meijers WC, van der Velde AR, Muller Kobold AC, et al. Variability of biomarkers in patients with chronic heart failure and healthy controls. Eur J Heart Fail 2017;19(3):357-65.

93. Ouwerkerk W, Voors AA, Zwinderman AH. Factors influencing the predictive power of models for predicting mortality and/or heart failure hospitalization in patients with heart failure. JACC Heart Fail 2014;2(5):429-36.

94. Rahimi K, Bennett D, Conrad N, et al. Risk prediction in patients with heart failure: a systematic review and analysis. JACC Heart Fail 2014;2(5):440-6.

95. Oliver JM, Gallego P, Gonzalez AE, et al. Impact of age and sex on survival and causes of death in adults with congenital heart disease. Int J Cardiol 2017.

96. Kajimoto K, Minami Y, Sato N, et al. Gender Differences in Anemia and Survival in Patients Hospitalized for Acute Decompensated Heart Failure With Preserved or Reduced Ejection Fraction. Am J Cardiol 2017;120(3):435-42. 


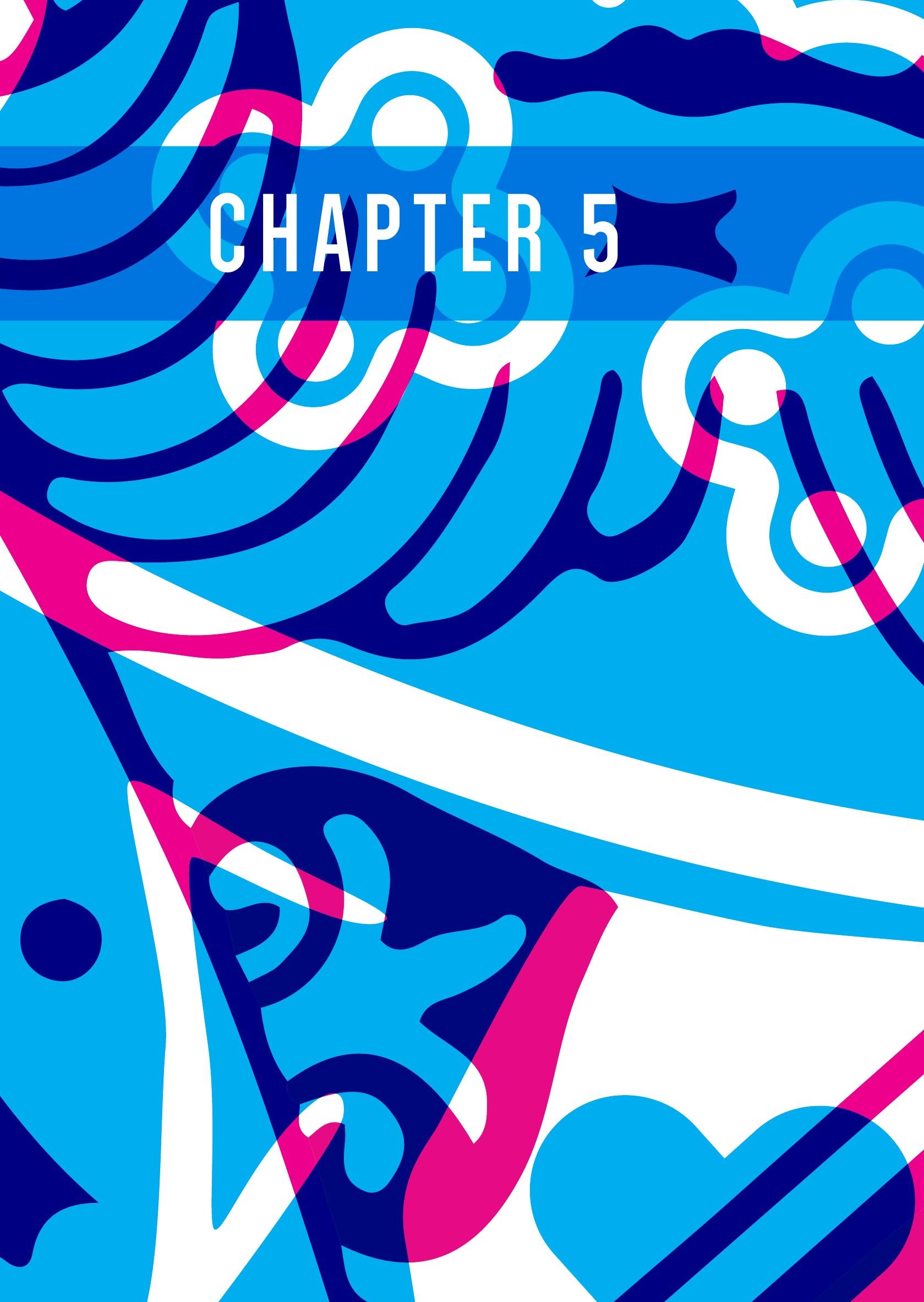




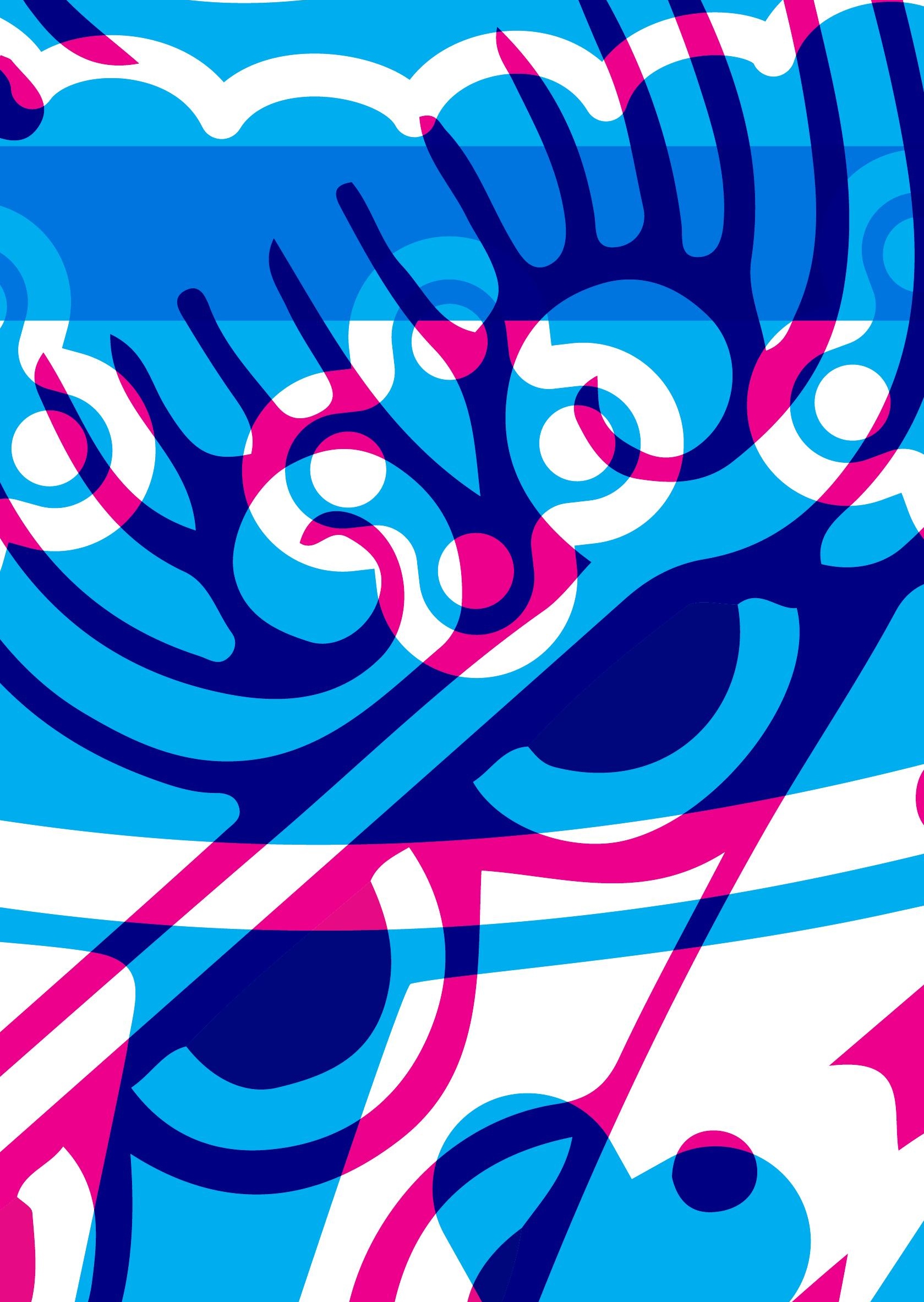




\section{CHAPTER 5}
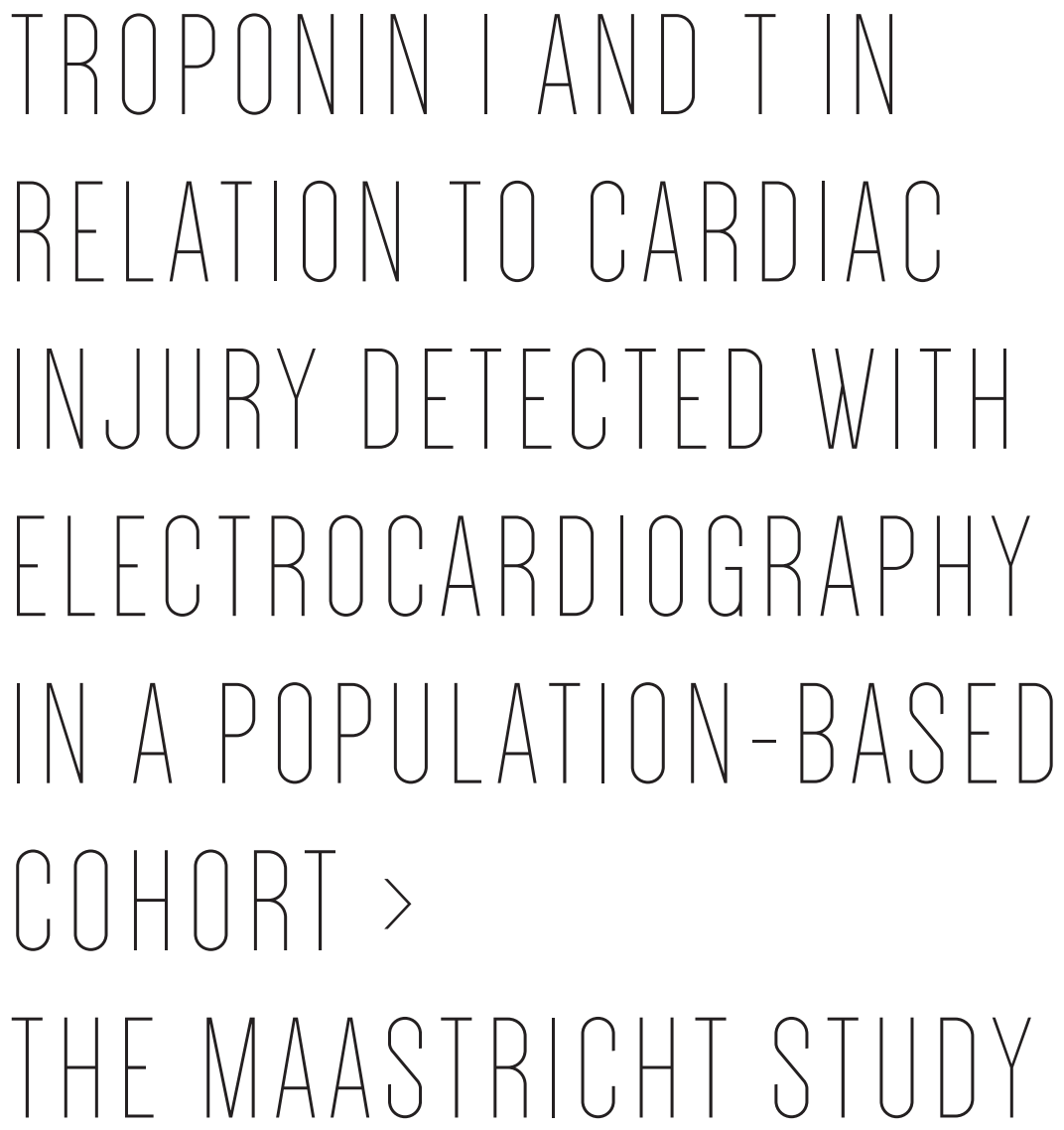

Dorien M Kimenai, Remy JH Martens, Jeroen P Kooman, Coen DA Stehouwer, Frans ES Tan, Nicolaas C Schaper, Pieter C Dagnelie, Miranda T Schram, Carla JH van der Kallen, Simone JS Sep, Jeroen DE van Suijlen, Abraham A Kroon, Otto Bekers, Marja P van Dieijen-Visser, Ronald MA Henry, Steven JR Meex 


\section{ABSTRACT}

\section{$>$ INTRODUCTION}

Interest in high-sensitivity cardiac troponin I (hs-cTnI) and $\mathrm{T}$ (hs-cTnT) has expanded from acute cardiac care to cardiovascular disease (CVD) risk stratification. Whether hs-cTnI and hs-cTnT are interchangeable in the ambulant setting is largely unexplored. Cardiac injury is a mechanism that may underlie the associations between troponin levels and mortality in the general population.

\section{$>$ MATERIAL AND METHODS}

In the population-based Maastricht Study, we assessed the correlation and concordance between hs-cTnI and hs-cTnT. Multiple regression analyses were conducted to assess the association of hs-cTnI and hs-cTnT with electrocardiographic (ECG) changes indicative of cardiac abnormalities.

\section{$>$ RESULTS}

In 3016 eligible individuals (mean age, $60 \pm 8$ years; $50.6 \%$, men) we found a modest correlation between hs-cTnI and hs-cTnT $(\mathrm{r}=0.585)$. After multiple adjustment, the association with ECG changes indicative of cardiac abnormalities was similar for both hs-cTn assays (OR, hs-cTnI: 1.72, 95\% CI: 1.40 - 2.10; OR, hs-cTnT: 1.6o, 95\% CI: $1.22-2.11$ ). The concordance of dichotomized hs-cTnI and hs-cTnT was $\mathbf{K}=0.397$ ( $\geq$ sex-specific $75^{\text {th }}$ percentile). Isolated high levels of hs-cTnI were associated with ECG changes indicative of cardiac abnormalities (OR: 1.93, 95\% CI: 1.01 - 3.68), whereas isolated high levels of hs-cTnT were not (OR: 1.07, 95\% CI: $0.49-2.31$ ).

\section{$>$ CONCLUSION}

In conclusion, there is a moderate correlation and limited concordance between hs-cTnI and hs-cTnT under non-acute conditions. These data suggest that associations of hs-cTnI and hs-cTnT with cardiac injury detected by ECG are driven by different mechanisms. This information may benefit future development of CVD risk stratification algorithms. 


\section{INTRODUCTION}

The cardiac troponins I and $\mathrm{T}$ are the preferred biomarkers for diagnosing acute myocardial infarction (AMI). ${ }^{1}$ Whereas conventional troponin assays were limited to the detection of plasma troponin concentrations that are typically seen in the setting of acute cardiac injury, high-sensitivity assays can detect cardiac troponin concentrations below the clinical cut-off levels. ${ }^{2,3}$ In parallel with this technical progress, interest in cardiac troponin has expanded from acute cardiac care to cardiovascular disease (CVD) risk stratification. Indeed, epidemiological studies have demonstrated that basal levels of high-sensitivity cardiac troponin I (hs-cTnI) and $\mathrm{T}$ (hs-cTnT) are invariably associated with cardiac morbidity and mortality, even in apparently healthy individuals. ${ }^{4-17}$

Whether hs-cTnI and hs-cTnT are interchangeable in CVD risk stratification algorithms is largely unexplored. An important issue here is the fact that, although during and after AMI, hs-cTnI and hs-cTnT levels in blood are strongly correlated (reported $\mathrm{r}=0.85),{ }^{13}$ and carry equivalent diagnostic value, ${ }^{18}$ they not do so under non-acute conditions (reported $r=0.44-0.70) \cdot{ }^{13,19-21}$ This surprising discrepancy may suggest different mechanisms of release or clearance for troponin I and $\mathrm{T}$ in the chronic setting, but more importantly, it raises the hypothesis that associations of cardiac troponin I and $\mathrm{T}$ with cardiac morbidity and mortality may be different.

Cardiac injury is a plausible mechanism that may underlie the associations between basal troponin levels and cardiac morbidity and mortality in the general population, and more insight in the relationship between cardiac troponin I and $\mathrm{T}$ with cardiac injury can provide useful data for cardiovascular risk prediction. ${ }^{22}$ Electrocardiography (ECG) changes are possibly indicative of cardiac injury (e.g., cardiac ischemia).

To test the hypothesis that the modest hs-cTnI-hs-cTnT correlation also translates into different associations with cardiac injury, we directly compared the associations of hscTnI and hs-cTnT with cardiac injury detected with ECG in a population-based cohort. In addition, we explored whether hs-cTnIand hs-cTnT provideinterchangeableinformation, in terms of their association with ECG changes indicative of cardiac abnormalities.

\section{METHODS}

\section{> STUDY POPULATION}

We used data from The Maastricht Study, an ongoing observational prospective population-based cohort study. The rationale and methodology have been described previously. ${ }^{23}$ In brief, the study focuses on the etiology, pathophysiology, complications and comorbidities of $\mathrm{T}_{2} \mathrm{DM}$ and is characterized by an extensive phenotyping approach. Eligible for participation were all individuals aged between 40 and 75 years and living in the southern part of the Netherlands. Participants were recruited through mass media 
campaigns and from the municipal registries and the regional Diabetes Patient Registry via mailings. Recruitment was stratified according to known T2DM status, with an oversampling of individuals with $\mathrm{T}_{2} \mathrm{DM}$, for reasons of efficiency.

The present report includes cross-sectional data from the first 3451 participants, who completed the baseline survey between November 2010 and September 2013. The examinations of each participant were performed within a time window of three months. The study has been approved by the institutional medical ethical committee (NL31329.068.10) and the Minister of Health, Welfare and Sports of the Netherlands (Permit 131088-105234-PG). All participants gave written informed consent. The study was performed in accordance with the Declaration of Helsinki. ${ }^{24}$

For the present study, individuals with self-reported history of AMI, type 1 diabetes mellitus (T1DM), or missing data on one or more of the independent variables or dependent variables were excluded. Self-reported history of AMI was examined using the Rose questionnaire. ${ }^{25}$

\section{$>$ DEFINITION OF ECG CHANGES INDICATIVE OF CARDIAC ABNORMALITIES}

A resting 12-lead ECG was obtained using the Mac 5500 ECG system (GE Medical Systems, Milwaukee, Wisconsin, USA). ECG data were processed automatically and coded according to the Minnesota code classification system (Fysiologic ECG Services B.V., Amsterdam, the Netherlands). ${ }^{26-28}$

Outcomes of interests were ECG changes indicative of cardiac abnormalities, classified according to the Whitehall criteria, which are indicating the relative likelihood of signs of cardiac ischemia. ${ }^{29}$ ECG changes indicative of cardiac abnormalities were categorized as "probable", "possible" or "unlikely". 29 "Probable" was attributed in case of major or medium abnormalities in Q or QS patterns or left bundle branch block (Minnesota coding; 1.1.1-1.2.8 or 7.1.1-7.1.2); "possible" was used for borderline Q/QS waves, or ST-segment abnormalities accompanied by abnormal T-waves (Minnesota coding; 1.3.1, 1.3.2 or 4.1.1, 4.1.2, 4.2, 4.3 accompanied by 5.1, 5.2, 5.3); and "unlikely" for all other Minnesota coding categories (Figure 5.1).

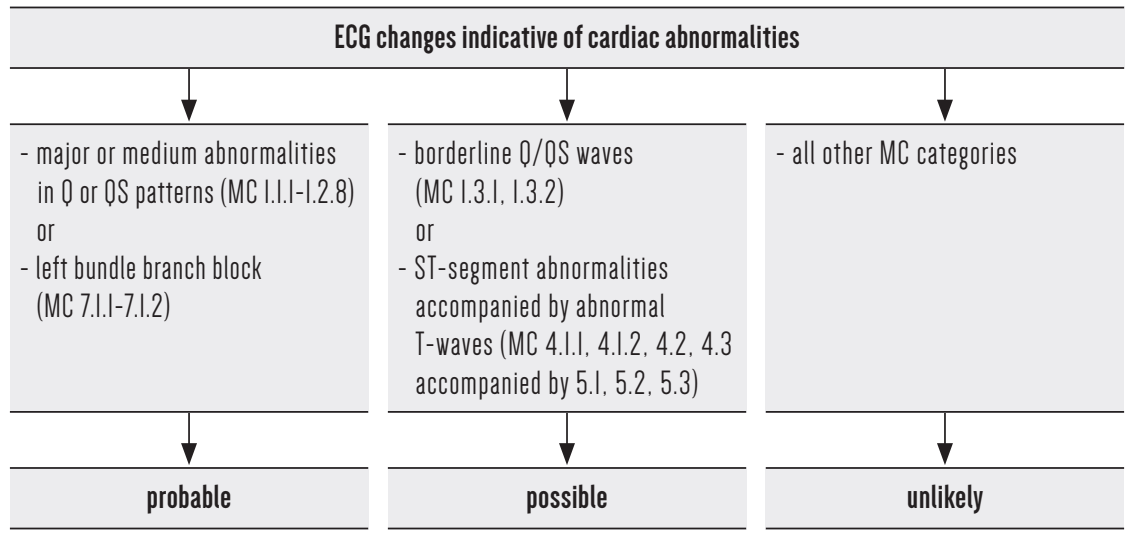




\section{BIOMARKER MEASUREMENTS}

Fasting blood samples were collected, centrifuged, and serum was stored in aliquots at $-80{ }^{\circ} \mathrm{C}$. Storage time prior to analyses varied from $1-4$ years and was identical for hs-cTnI and hs-cTnT. Cardiac troponin I and T were measured in serum using the ARCHITECT i2ooo SR analyzer (Abbott Diagnostics, Lake Forest, IL, USA) and Roche Cobas-6ooo analyzer (F. Hoffman-La Roche Ltd, Basel, Switzerland), respectively. For hs-cTnI the limit of blank (LoB) ranged from 0.7 to $1.3 \mathrm{ng} / \mathrm{L}$, the LoD ranged from 1.1 to $1.9 \mathrm{ng} / \mathrm{L}$ and the LoQ ranged from 4.0 to 10 $\mathrm{ng} / \mathrm{L}$ (package insert). For hs-cTnT the LoB was $3 \mathrm{ng} / \mathrm{L}$, the LoD was $5 \mathrm{ng} / \mathrm{L}$ and the LoQ was $13 \mathrm{ng} / \mathrm{L}$ (package insert)..$^{30}$ The hs-cTnI assay achieves a $10 \% \mathrm{CV}$ at 4.7 $\mathrm{ng} / \mathrm{L}$ and a $20 \% \mathrm{CV}$ at $1.3 \mathrm{ng} / \mathrm{L}$. The hs-cTnT assay achieves a $10 \% \mathrm{CV}$ at $13 \mathrm{ng} / \mathrm{L}$ and a $20 \% \mathrm{CV}$ at $6.8 \mathrm{ng} / \mathrm{L}$ (package insert). ${ }^{\circ}$ Cystatin $\mathrm{C}$ and creatinine were measured in all serum samples using the Roche Cobas-80oo analyzer (F. Hoffman-La Roche Ltd, Basel, Switzerland) and eGFR was calculated with the Chronic Kidney Disease Epidemiology Collaboration (CKD-EPI) equation based on both cystatin C and creatinine. ${ }^{31}$

\section{$>$ POTENTIAL CONFOUNDERS}

We assessed glucose metabolism status, eGFR, smoking behavior, total-to-HDL cholesterol ratio, triglyceride levels, lipid-modifying medication, office or $24 \mathrm{~h}$ average ambulatory systolic blood pressure, antihypertensive medication, waist-tohip ratio, alcohol consumption and educational level as described previously. ${ }^{23,32,33}$

\section{$>$ STATISTICAL ANALYSIS}

Continuous variables were expressed as mean and standard deviation (SD), or median and interquartile range (IQR) for non-Gaussian distributions. Categorical data were reported as $\mathrm{n}(\%)$.

The correlation between natural log-transformed (ln) hs-cTnI and ln hs-cTnT was assessed by Pearson's correlation test. Cohen's kappa (к) was used to assess the concordance between the hs-cTnI and hs-cTnT assays, and the $95 \%$ confidence interval (CI) around $\mathbf{\kappa}$ was calculated using bias-corrected accelerated bootstrapping (resampling with replacement: 5000 bootstrap replicates).

Multivariable logistic regression analyses were conducted to quantify the associations between both troponins and ECG changes indicative of cardiac abnormalities. In the primary analyses, the ECG abnormality categories "possible" and "unlikely" were combined into a single reference category. To allow for a direct comparison of troponin I and $\mathrm{T}$, the independent variables hs-cTnI and hs-cTnT were tested separately in the statistical models as described below. The hs-cTn values below the LoB were set equal to the LoB value (hs-cTnI, $0.9 \mathrm{ng} / \mathrm{L}$ : hscTnT, $3 \mathrm{ng} / \mathrm{L}$ )/2. For hs-cTnI a LoB value of $0.9 \mathrm{ng} / \mathrm{L}$ was chosen to obtain similar proportions for troponin I and $\mathrm{T}$ of participants with values above the LoB. Accordingly, hs-cTnI and hs-cTnT were natural log-transformed and examined 
per 1-SD increase in the regression model. The independent variables (hs-cTnI, hs-cTnT) were analyzed in four different models with sequential adjustment for potential confounders; model 1: crude model; model 2: model 1 + sex, age, glucose metabolism status; model 3: model $2+$ eGFR; model 4A: model $3+$ smoking behavior, total-to-HDL cholesterol ratio, triglyceride levels, lipid-modifying medication, office systolic blood pressure, antihypertensive medication, waistto-hip ratio, alcohol consumption, educational level; model $4 \mathrm{~B}$ : model $4 \mathrm{~A}$ with replacement of office systolic blood pressure by $24 \mathrm{~h}$ average ambulatory systolic blood pressure. Because of the design of The Maastricht Study, we tested for interaction between hs-cTn and glucose metabolism status. Interaction was considered significant when the $\mathrm{p}$ value ( $\left.\mathrm{p}_{\text {interaction }}\right)$ of the interaction term was < o.10. No significant interaction was found for glucose metabolism status and all analyses were, therefore, conducted unstratified.

In secondary analyses, we set the hs-cTn values below LoD, $20 \% \mathrm{CV}, 10 \% \mathrm{CV}$ and LoQ equally to these lower limits of measurements. Furthermore, to allow for a more liberal definition of ECG changes indicative of cardiac abnormalities, "possible" and "probable" were combined as one outcome category and compared with the reference category "unlikely". All statistical analyses were performed using SPSS for windows 23.o (IBM Corp., Armonk, NY, USA).

\section{$>$ DATA AVAILABILITY}

The datasets generated during and/or analyzed during the current study are not publicly available due to privacy issues and national laws but are available from the management board of The Maastricht Study on reasonable request under the provision that data may not leave the university / hospital premises.

\section{RESULTS}

\section{> STUDY POPULATION}

A total of 3016 eligible participants from The Maastricht Study cohort were included in the present study (Supplemental Figure 5.1). Clinical characteristics of the total study population, and stratified by the presence of ECG changes indicative of cardiac abnormalities, are shown in Table 5.1.

The overall proportion of participants with detectable hs-cTnT levels (Limit of Detection (LoD) $\geq 5 \mathrm{ng} / \mathrm{L}$ ) was $54 \%$. The overall proportion of detectable hs-cTnI levels ranged from $51 \%(\mathrm{LoD} \geq 1.9 \mathrm{ng} / \mathrm{L})$ to $83 \%(\mathrm{LoD} \geq 1.1 \mathrm{ng} / \mathrm{L})$. The overall proportion participants with hs-cTn levels above the 10\% coefficient of variation (CV) for hscTnT and hs-cTnI were $6 \%$ and $10 \%$, respectively.

CVD risk factors were more prevalent in participants with ECG changes indicative of cardiac abnormalities as compared with participants without ECG changes 
indicative of cardiac abnormalities (Table 5.1). The number (\%) of Minnesota coding categories of ECG abnormalities of the total study population are shown in Supplemental Table 5.1.

\section{$>$ MODEST CORRELATION BETWEEN HS-CTNI AND HS-CTNT}

In contrast to the acute cardiac care setting where troponin $\mathrm{I}$ and $\mathrm{T}$ are interchangeable ${ }^{18}$, a remarkably modest correlation was found between hs-cTnI and hs-cTnT in these ambulatory participants $(\mathrm{r}=0.585,95 \% \mathrm{CI} 0.562-0.608$, $\mathrm{p}<0.001$, Figure 5.2). The modest correlation was not due to discrepant sensitivities of hs-cTn assays. In the analyses we corrected for the proportion of participants below the LoB, and even after excluding participants with hs-cTn below the LoD (LoD hscTnI: $1.9 \mathrm{ng} / \mathrm{L}$, LoD hs-cTnT: $5 \mathrm{ng} / \mathrm{L}$ ), which equalized the proportion of participants with detectable hs-cTnT levels to those of the hs-cTnI assay, the correlation did not improve $(\mathrm{r}=0.373,95 \% \mathrm{CI} 0.322-0.420, \mathrm{p}<0.001, \mathrm{n}=118 \mathrm{o})$.

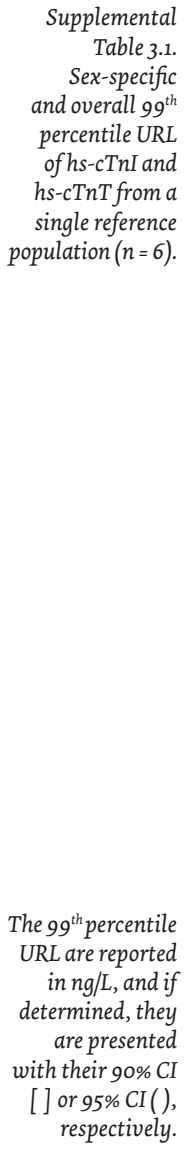

\begin{tabular}{|c|c|c|c|}
\hline variable & $\begin{array}{l}\text { total study population } \\
(n=3016)\end{array}$ & $\begin{array}{l}\text { no ECG changes } \\
\text { indicative of cardiac } \\
\text { abnormalities } \\
(n=2923)\end{array}$ & $\begin{array}{l}\text { ECG changes } \\
\text { indicative of cardiac } \\
\text { abnormalities } \\
(n=93)\end{array}$ \\
\hline age (years) & $60(8)$ & $60(8)$ & $63(8)$ \\
\hline sex (male) & $1526(50.6 \%)$ & $1479(50.6 \%)$ & $47(50.5 \%)$ \\
\hline $\operatorname{BMI}\left(\mathrm{kg} / \mathrm{m}^{2}\right)$ & $26.9(4.5)$ & $26.9(4.5)$ & $28.4(5.0)$ \\
\hline \multicolumn{4}{|l|}{ eGFR } \\
\hline$<60 \mathrm{~mL} / \mathrm{min} / .73 \mathrm{~m}^{2}$ & $\log (3.6 \%)$ & $103(3.5 \%)$ & $6(6.5 \%)$ \\
\hline $60-<90 \mathrm{~mL} / \mathrm{min} / 1.73 \mathrm{~m}^{2}$ & $1438(47.7 \%)$ & |393 (47.7\%) & $45(48.4 \%)$ \\
\hline$\geq 90 \mathrm{~mL} / \mathrm{min} / .73 \mathrm{~m}^{2}$ & $1469(48.7 \%)$ & |427 (48.8\%) & $42(45.2 \%)$ \\
\hline \multicolumn{4}{|l|}{ glucose metabolism } \\
\hline NGM & $1776(58.9 \%)$ & $1739(59.5 \%)$ & $37(39.8 \%)$ \\
\hline IFG & $129(4.3 \%)$ & $125(4.3 \%)$ & $4(4.3 \%)$ \\
\hline IGT & $330(10.9 \%)$ & $312(10.7 \%)$ & $18(19.4 \%)$ \\
\hline T2DM & $781(25.9 \%)$ & $747(25.6 \%)$ & $34(36.6 \%)$ \\
\hline office BP, systolic (mmHg) & $135(18)$ & $134(18)$ & $140(17)$ \\
\hline office BP, diastolic (mmHg) & $76(10)$ & $76(10)$ & $77(11)$ \\
\hline hypertension (yes) & $1628(54.0 \%)$ & $1557(53.3 \%)$ & $71(76.3 \%)$ \\
\hline antihypertensive medication (yes) & $1108(36.7 \%)$ & $1056(36.1 \%)$ & $52(55.9 \%)$ \\
\hline \multicolumn{4}{|l|}{ smoking behavior } \\
\hline never & $1090(36.1 \%)$ & $1055(36.1 \%)$ & $35(37.6 \%)$ \\
\hline former & $1525(50.6 \%)$ & $1476(50.5 \%)$ & $49(52.7 \%)$ \\
\hline current & $401(\mid 3.3 \%)$ & $392(\mid 3.4 \%)$ & $9(9.7 \%)$ \\
\hline total-to-HDL cholesterol ratio & $3.5(2.8-4.3)$ & $3.5(2.8-4.3)$ & $3.4(2.7-4.4)$ \\
\hline triglycerides $(\mathrm{mmol} / \mathrm{L})$ & $1.2(0.9-1.7)$ & $1.2(0.9-1.7)$ & $1.3(0.9-1.6)$ \\
\hline lipid-modifying medication (yes) & 992 (32.9\%) & $943(32.3 \%)$ & $49(52.7 \%)$ \\
\hline hs-cTnl (ng/L) & $1.9(1.2-3.0)$ & $1.8(1.2-2.9)$ & $2.8(1.6-4.9)$ \\
\hline hs-cTnT (ng/L) & $5.3(3.8-7.7)$ & $5.3(3.7-7.6)$ & $7.0(4.7-11.3)$ \\
\hline
\end{tabular}




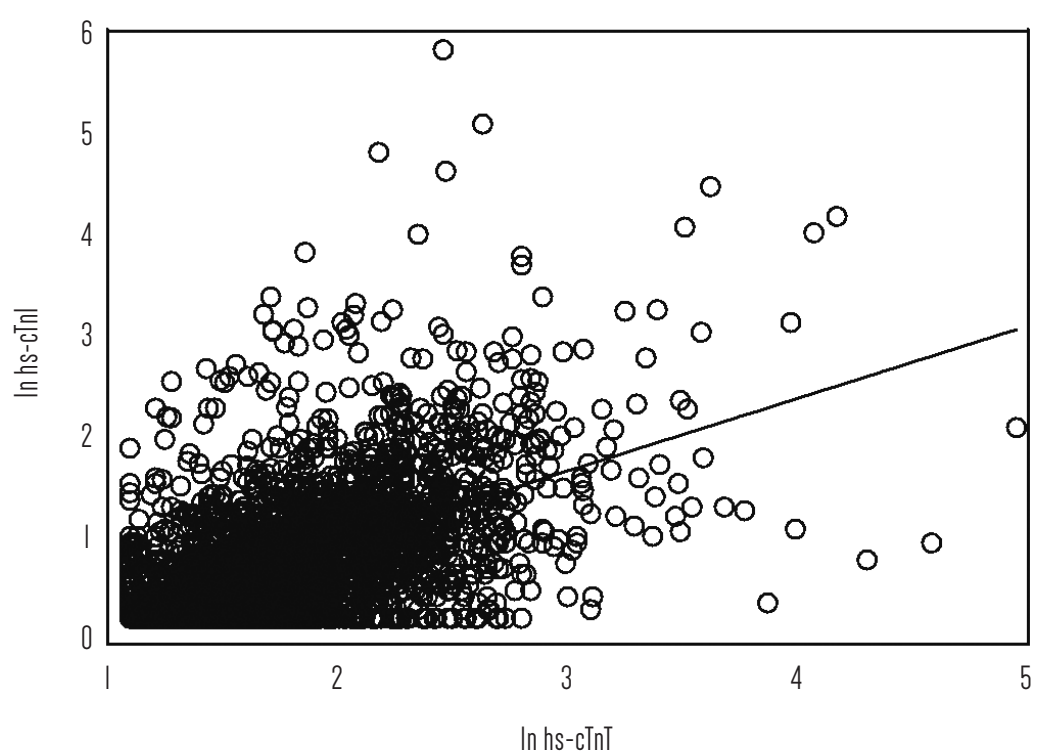

Figure 5.2.

Correlation between natural log transformed hs-cTnI and natural log transformed hs-cTnT ( $\beta=0.465,95 \%$ CI $0.442-0.488$, $p<0.001$, $\left.R^{2}=0.343\right)$.

\section{HS-CTNI AND HS-CTNT ARE INDEPENDENTLY ASSOCIATED WITH CARDIAC INJURY DETECTED WITH ECG}

To test whether the modest hs-cTnI-hs-cTnT correlation also translated into different associations with cardiac injury, we directly compared the associations of hs-cTnI and hs-cTnT with cardiac injury detected with ECG. Univariable associations of hs-cTnI and hs-cTnT with ECG changes indicative of cardiac abnormalities were statistically significant and numerically similar (Table 5.2, model 1). Sequential adjustments for demographic variables, eGFR and traditional CVD risk factors did not abrogate the similar associations of both hs-cTn with ECG changes indicative of cardiac abnormalities (odds ratio (OR) per 1-SD higher ln hscTnI: 1.72, 95\% CI 1.40 - 2.10; OR per 1-SD higher ln hs-cTnT: 1.6o, 95\% CI 1.22 - 2.11) (Table 5.2, model 4A).

Results were robust in sensitivity analyses, of which values below the LoD, $20 \% \mathrm{CV}, 10 \% \mathrm{CV}$, and LoQ were set equal to these lower limits of measurements (Supplemental Table 5.2). However, the association between hs-cTnT and ECG changes indicative of cardiac abnormalities remained not statistically significant when hs-cTnT values below $13 \mathrm{ng} / \mathrm{L}$ were set at $13 \mathrm{ng} / \mathrm{L}$ (10\% CV, LoQ) (Supplemental Table 5.2). Results were not materially altered with a more liberal definition of ECG changes indicative of cardiac abnormalities (definition included also borderline Q/QS waves, or ST-segment abnormalities accompanied by abnormal T-waves) (Supplemental Table 5.3). 


\begin{tabular}{|c|c|c|c|c|c|}
\hline \multirow{3}{*}{$\begin{array}{r}\text { Table 5.2. } \\
\text { Associations } \\
\text { of hs-cTnI and } \\
\text { hs-cTnT with } \\
\text { ECG changes } \\
\text { indicative } \\
\text { of cardiac } \\
\text { abnormalities. }\end{array}$} & & \multicolumn{2}{|c|}{ hs-cTnl (In, I-SD increase) } & \multicolumn{2}{|c|}{ hs-cTnT (In, I-SD increase) } \\
\hline & & OR (95\% CI) & p-value & OR (95\% CI) & p-value \\
\hline & modell & $1.72(1.45-2.05)$ & $<0.001$ & $1.67(1.36-2.05)$ & $<0.001$ \\
\hline & model 2 & $1.72(1.42-2.09)$ & $<0.001$ & $1.56(1.22-2.01)$ & 0.001 \\
\hline & model 3 & $1.76(1.45-2.14)$ & $<0.001$ & $1.67(1.28-2.18)$ & $<0.001$ \\
\hline & model $4 \mathrm{~A}$ & $1.72(1.40-2.10)$ & $<0.001$ & 1.60 (I.22-2..I) & 0.001 \\
\hline & model 4B & $1.89(1.53-2.34)$ & $<0.001$ & $1.65(1.24-2.20)$ & 0.001 \\
\hline
\end{tabular}

Model 1: crude model; model 2: model $1+$ sex, age, glucose metabolism status; model 3: model $2+e G F R ;$ model 4A: model 3 + smoking behavior, total-to-HDL cholesterol ratio, triglyceride levels, lipid-modifying medication, office systolic blood pressure, antihypertensive medication, waist-to-hip ratio, alcohol consumption, educational level; model $4 \mathrm{~B}:$ model $4 \mathrm{~A}$ with replacement of office systolic blood pressure by 24 h average ambulatory systolic blood pressure.

Both hs-cTnI and hs-cTnT showed no significant interaction with eGFR on the association with ECG changes indicative of cardiac abnormalities (hs-cTnI * eGFR, $\mathrm{p}_{\text {interaction }}=0.991 ;$ hs-cTnT * eGFR, $\mathrm{p}_{\text {interaction }}=0.409$ ).

\section{$>$ DISCORDANCE OF HS-CTNI AND HS-CTNT ASSAYS}

Study participants were classified into four groups on the basis of dichotomized hs-cTn concentrations, divided at the sex-specific $75^{\text {th }}$ percentile (hs-cTnI, women: $2.20 \mathrm{ng} / \mathrm{L}$; hs-cTnI, men: $3.70 \mathrm{ng} / \mathrm{L}$; hs-cTnT, women: $5.55 \mathrm{ng} / \mathrm{L}$; hs-cTnT, men: 9.36 $\mathrm{ng} / \mathrm{L}$ ). Dichotomization at the $75^{\text {th }}$ percentile was based on a troponin I threshold effect corresponding to the upper quartile. This threshold effect for troponin I was first described in relation to cardiovascular death and heart failure ${ }^{19}$ and now further extended in this study in relation to the presence of ECG changes indicative of cardiac abnormalities (Figure 5.3).
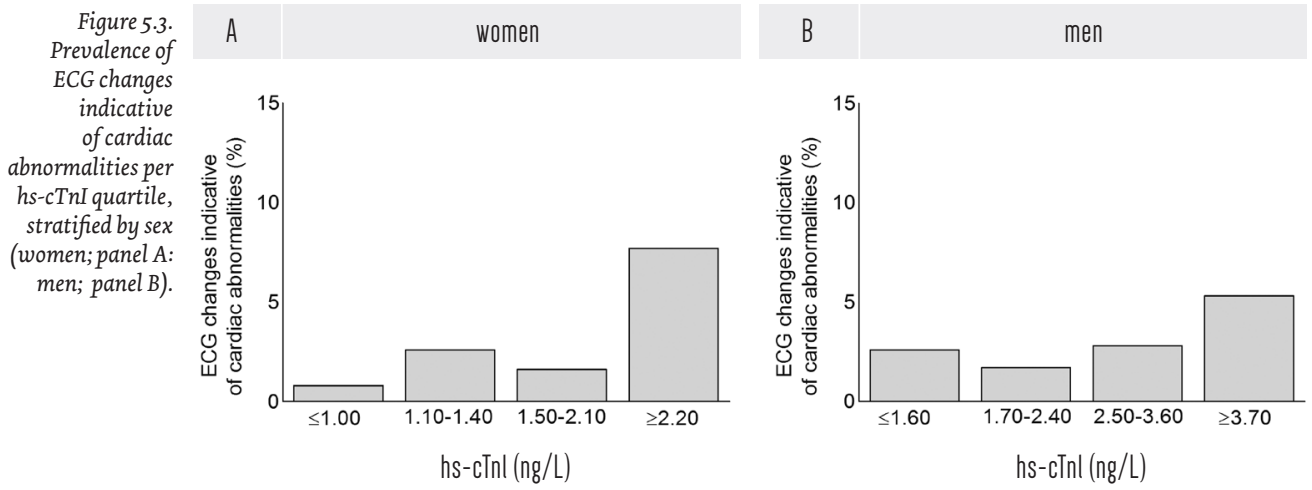
Clinical characteristics of the groups based on sex-specific $75^{\text {th }}$ percentiles (and sex-specific $99^{\text {th }}$ percentiles) per hs-cTn assay are shown in Supplemental Table 5.4 and 5.5. The concordance between the two assays was statistically significant but rather weak (Cohen's kappa (к) 0.397; 95\% CI 0.359 - 0.434), with $22.4 \%$ of participants having a discordant classification (Table 5.3).

\begin{tabular}{|c|c|c|c|c|}
\hline & & \multicolumn{3}{|c|}{ hs-cInT $\cong$ sex-specific $75^{\text {th }}$ percentile } \\
\hline & & no & yes & total \\
\hline \multirow{3}{*}{$\begin{array}{l}\text { hs-cTnl } \\
\geq \text { sex-specific } \\
75^{\text {th }} \text { percentile }\end{array}$} & \multirow[t]{2}{*}{ no } & \multirow[t]{2}{*}{$1934(64.1 \%)$} & \multirow[t]{2}{*}{$344(11.4 \%)$} & \multirow[t]{2}{*}{$2278(75.5 \%)$} \\
\hline & & & & \\
\hline & yes & 332 (11.0\%) & $406(13.5 \%)$ & $738(24.5 \%)$ \\
\hline & total & $2266(75.1 \%)$ & $750(24.9 \%)$ & $3016(100 \%)$ \\
\hline
\end{tabular}

Table 5.3. Concordance of hs-cTnI and hs-cTnT according to sex-specific $75^{\text {th }}$ percentile thresholds.

Data are reported as $n(\%)$. Cohen's $\mathbf{K}=0.397$ (95\% CI 0.359-0.434). Four groups were classified according to sex-specific $75^{\text {th }}$ percentiles of hs-cTnI and hs-cTnT (hs-cTnI, women: $2.20 \mathrm{ng} / \mathrm{L}$; hs-cTnI, men: $3.70 \mathrm{ng} / \mathrm{L}$; hs-cTnT, women: $5.55 \mathrm{ng} / \mathrm{L}$; hs-cTnT, men: $9.36 \mathrm{ng} / \mathrm{L}$ ).

We then assessed the associations of dichotomized hs-cTn concentrations with ECG changes indicative of cardiac abnormalities. Both high levels of hs-cTnI and hscTnT were strongly associated with ECG changes indicative of cardiac abnormalities (Figure 5.4). 


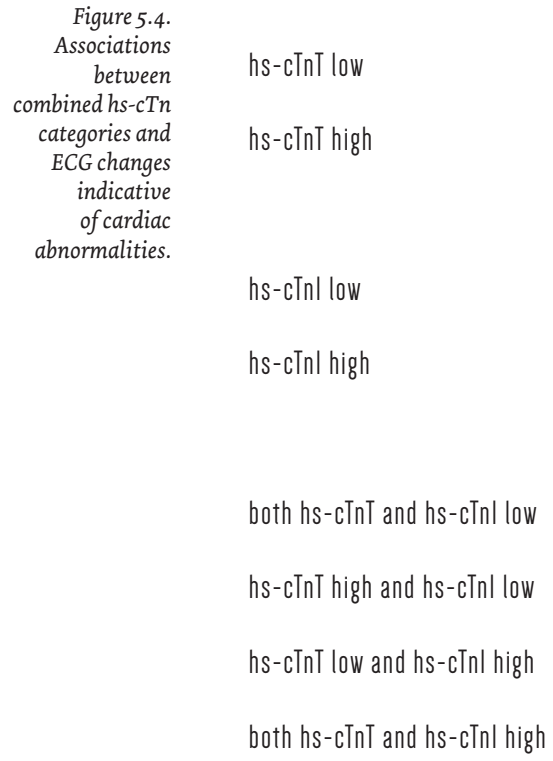

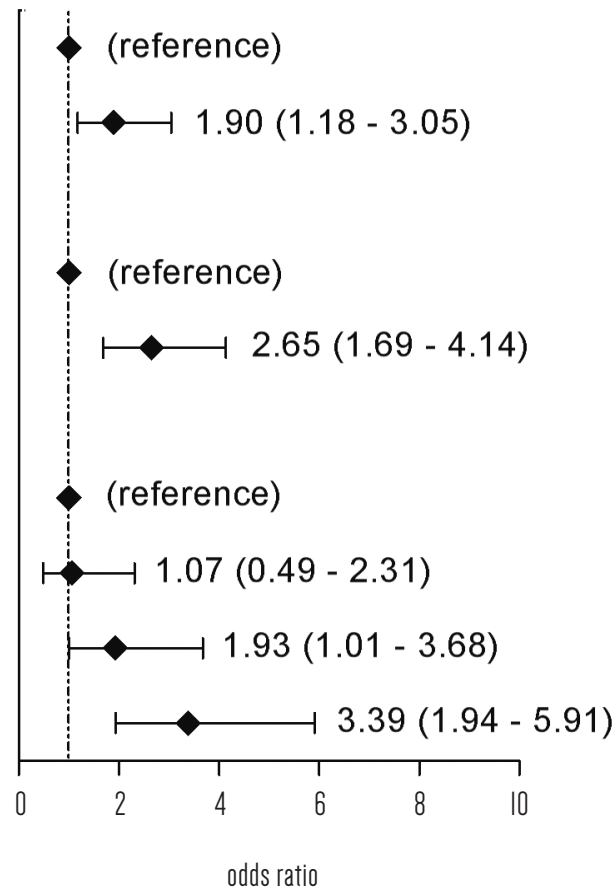

Associations between combined hs-cTn categories and ECG changes indicative of cardiac abnormalities. Definitions of "low" and "high" hs-cTn categories were based on the sex-specific $75^{\text {th }}$ percentiles of hs-cTnI and hs-cTnT (hs-cTnI, women: $2.20 \mathrm{ng} / \mathrm{L}$; hs-cTnI, men:3.70 $\mathrm{ng} / \mathrm{L}$; hs-cTnT, women: $5.55 \mathrm{ng} / \mathrm{L}$; hs-cTnT, men: $9.36 \mathrm{ng} / \mathrm{L}$ ). Category "low" included participants with hs-cTn levels $<$ the sex-specific $75^{\text {th }}$ percentile and category "high" included participants $\geq$ the sex-specific $75^{\text {th }}$ percentile. Model was adjusted for sex, age, glucose metabolism status, eGFR, smoking behavior, total-to-HDL cholesterol ratio, triglyceride levels, lipid-modifying medication, office systolic blood pressure, antihypertensive medication, waist-to-hip ratio, alcohol consumption and educational level.

The strongest association with ECG changes indicative of cardiac abnormalities was observed for participants who had high levels of both hs-cTnI and hs-cTnT ("both hs-cTnT and hs-cTnI high" as compared with the reference category "both hs-cTnT and hs-cTnI low", OR 3.39, 95\% CI 1.94 - 5.91, p < 0.001) (Figure 5.4). As compared with the category "both hs-cTnT and hs-cTnI low", isolated high levels of troponin I were associated with ECG changes indicative of cardiac abnormalities (OR 1.93, 95\% CI 1.01 - 3.68, $\mathrm{p}=0.046$ ), whereas isolated high levels of troponin T were not (OR 1.07, $95 \% \mathrm{CI} 0.49-2.31, \mathrm{p}=0.869$ ) (Figure 5.4).

These results were robust in a sensitivity analysis in which we used a more liberal definition of ECG changes indicative of cardiac abnormalities (Supplemental Figure 5.2), and when we applied a range of alternative percentile cut-off points (percentile $66^{\text {th }}$ to percentile $90^{\text {th }}$ ) to dichotomize cardiac troponin levels (Supplemental Table 5.6). 


\section{> CLINICAL CHARACTERISTICS OF DISCORDANTLY CLASSIFIED PARTICIPANTS}

In an exploratory analysis to further investigate the discordance between both hs-cTn assays, we compared clinical parameters of concordant and discordantly classified participants. Discordantly classified participants differed most markedly in estimated glomerular filtration rate (eGFR) and the prevalence of type 2 diabetes mellitus (T2DM): prevalence of eGFR $<60 \mathrm{~mL} / \mathrm{min} / 1.73 \mathrm{~m}^{2}$ was $7.6 \%$ in participants with isolated high hs-cTnT levels compared with $1.5 \%$ for participants with isolated high hs-cTnI levels (Supplemental Table 5.7). Prevalence of T2DM was $42.7 \%$ vs $20.5 \%$ in participants with isolated high hs-cTnT levels compared with participants with isolated high hs-cTnI levels, respectively.

\section{DISCUSSION}

This study reports four major findings. 1) Hs-cTnI and hs-cTnT were only moderately correlated in the general population. 2) There was substantial discordance between hs-cTnI and hs-cTnT under non-acute conditions. 3) Higher hs-cTnI and hs-cTnT were similarly associated with ECG changes indicative of cardiac abnormalities independent of demographic variables, eGFR and CVD risk factors, and 4) isolated high levels of troponin I, but not isolated high levels of troponin $\mathrm{T}$, were associated with ECG changes indicative of cardiac abnormalities, independent of the same risk factors as under 3 .

In contrast to the setting of acute coronary syndrome, where hs-cTnI and hscTnT are strongly correlated ${ }^{13}$ and are considered diagnostically interchangeable ${ }^{18}$, recent data suggest a much lower correlation under non-acute conditions such as atrium fibrillation ${ }^{20}$, stable coronary artery disease ${ }^{19}$, and chronic kidney disease ${ }^{21}$ (Supplemental Table 5.8). This may reflect different mechanisms that drive troponin I and T elevations in the chronic setting. The present results corroborate and extend previously reported data by showing substantial discordance between troponin I and $\mathrm{T}$ elevations in a population-based cohort.

This study focused on the relationship with cardiac injury, which is a possible mechanism for troponin I and $\mathrm{T}$ elevations in the ambulant setting. We found that isolated elevations of troponin I, but not isolated troponin $\mathrm{T}$ levels, were associated with ECG changes indicative of cardiac abnormalities. This would suggest that the similar strength of the association for hs-cTnI and hs-cTnT with ECG changes indicative of cardiac abnormalities are due to different underlying mechanisms. Hs-cTnI and hs-cTnT may therefore not be interchangeable as predictor for cardiovascular events and mortality in the ambulant setting, or on the other hand, they may even be complementary to each other. Unraveling the discrepancies between both hs-cTn may contribute to the development of CVD risk stratification algorithms that comprise cardiac biomarkers. 
The source of discordant hs-cTnI and hs-cTnT measurements can be analytical or biological. Differences in imprecision between hs-cTnI and hs-cTnT are an unlikely source of the modest correlation between hs-cTnI and hs-cTnT: when participants below an LoD of $1.9 \mathrm{ng} / \mathrm{L}$ were excluded, which brought the proportion of participants with measurable troponin I concentrations to a similar percentage as the troponin $\mathrm{T}$ assay (hs-cTnI 51\%, hs-cTnT 54\%), the correlation between troponin I and $\mathrm{T}$ became even weaker $(\mathrm{r}=0.373 \mathrm{vs} \mathrm{r}=0.585)$. Lack of analytical specificity due to interference of heterophilic or other antibodies is also unlikely to account for the substantial level of discordance and modest correlation between hs-cTnI and hs-cTnT. Hence, we speculate that true biological differences in release and/or elimination underlie the moderate correlation between hs-cTn assays in the chronic setting. We further explored which variables were associated with the distinct distribution patterns of troponin $\mathrm{T}$ and $\mathrm{I}$, and found that a reduced eGFR and T2DM were more prevalent in participants with isolated elevations of troponin $\mathrm{T}$, which is in line with the findings of Hijazi et al. ${ }^{20}$ Contrary to their findings, we observed no differences in age and proportion of men, but this is probably due to the age limit of 75 years of The Maastricht Study and the fact that we used sex-specific thresholds in our definition of elevated hs-cTn levels, while they did not. Whether these particular traits are mechanistically involved in distinct release and/or clearance patterns of troponin $\mathrm{T}$ and I is unknown. In this regard, previous studies have shown a stronger association of hs-cTnT than hs-cTnI with eGFR ${ }^{14,21,34-36}$, and our results expand these data by showing a higher prevalence of reduced eGFR in participants with isolated high hs-cTnT levels.

This study has several limitations. 1) The cross-sectional nature of our study precludes assessment of longitudinal associations of hs-cTnI and hs-cTnT with incident cardiac morbidity and mortality. Although the results are in line with longitudinal associations observed in a cohort with atrium fibrillation ${ }^{20}$, and extend this phenomenon to individuals from a population-based cohort, the next relevant step should be investigating whether troponin I and $\mathrm{T}$ are interchangeable predictors for cardiovascular morbidity and mortality in the general population. 2) Only one hs-cTnI assay was studied (Abbott), and the lack of standardization of hscTnI assays precludes extrapolation of our data to other troponin I assays. 3) The study participants were mainly of Caucasian origin, limiting generalizability to other ethnic groups. 4) The definition of cardiac injury is based on ECG data only, without additional information on cardiac ischemia from coronary imaging or echocardiography. Imaging modalities were not yet available in the current dataset. Although the selected ECG criteria indicate the relative likelihood of signs of cardiac ischemia, ECG abnormalities due to for e.g. left ventricular hypertrophy, dilated cardiomyopathy or amyloid cardiomyopathy cannot be excluded. 5) Although hscTnI and hs-cTnT were measured with high-sensitivity assays, concentrations were still below the LoB and LoD in a substantial number of participants. 6) To obtain a direct comparison between hs-cTnI and hs-cTnT assay, we corrected in the analysis for assay performance by adjustment of lower limits measurements. However, the 
lower sensitivity of hs-cTnT assay may have limited to show the association with hs-cTnT levels and ECG changes indicative of cardiac abnormalities.

In conclusion, this study showed a moderate correlation and limited concordance between the hs-cTnI and hs-cTnT assays. We showed that isolated high troponin I levels, but not isolated high troponin $\mathrm{T}$ levels, were independently associated with cardiac injury detected with ECG under non-acute conditions. These data suggest that observed associations of hs-cTnI and hs-cTnT with ECG changes indicative of cardiac abnormalities are driven by different mechanisms. This information may benefit future development of CVD risk stratification algorithms, using information from high-sensitivity cardiac troponin I and T assays. 
REFERENCES

1. Roffi, M. et al. 2015 ESC Guidelines for the management of acute coronary syndromes in patients presenting without persistent ST-segment elevation: Task Force for the Management of Acute Coronary Syndromes in Patients Presenting without Persistent STSegment Elevation of the European Society of Cardiology (ESC). European heart journal 37, 267-315, doi:10.1093/eurheartj/ehv320 (2016).

2. Apple, F. S., Ler, R. \& Murakami, M. M. Determination of 19 cardiac troponin I and T assay $99^{\text {th }}$ percentile values from a common presumably healthy population. Clinical chemistry 58, 1574-1581, doi:10.1373/clinchem.2012.192716 (2012).

3. Apple, F. S., Steffen, L. M., Pearce, L. A., Murakami, M. M. \& Luepker, R. V. Increased cardiac troponin I as measured by a high-sensitivity assay is associated with high odds of cardiovascular death: the Minnesota Heart Survey. Clinical chemistry 58, 930-935, doi:10.1373/clinchem.2011.179176 (2012).

4. Eggers, K. M., Venge, P., Lindahl, B. \& Lind, L. Cardiac troponin I levels measured with a high-sensitive assay increase over time and are strong predictors of mortality in an elderly population. Journal of the American College of Cardiology 61, 1906-1913, doi:10.1016/j. jacc.2012.12.048 (2013).

5. de Lemos, J. A. et al. Association of troponin T detected with a highly sensitive assay and cardiac structure and mortality risk in the general population. JAMA 304, 2503-2512, doi:10.1001/jama.2010.1768 (2010).

6. Hussein, A. A. et al. Cardiomyocyte injury assessed by a highly sensitive troponin assay and sudden cardiac death in the community: the Cardiovascular Health Study. Journal of the American College of Cardiology 62, 2112-2120, doi:10.1016/j.jacc.2013.07.049 (2013).

7. Eggers, K. M. et al. High-sensitive cardiac troponin $\mathrm{T}$ and its relations to cardiovascular risk factors, morbidity, and mortality in elderly men. American heart journal 166, 541-548, doi:10.1016/j.ahj.2013.07.004 (2013).

8. Oluleye, O. W. et al. Troponin T, B-type natriuretic peptide, C-reactive protein, and causespecific mortality. Ann Epidemiol 23, 66-73, doi:10.1016/j.annepidem.2012.11.004 (2013).

9. Masson, S. et al. The fibroblast growth factor-23 and Vitamin D emerge as nontraditional risk factors and may affect cardiovascular risk. J Intern Med 277, 318-330, doi:10.1111/ joim.12232 (2015).

10. Neumann, J. T. et al. Comparison of three troponins as predictors of future cardiovascular events--prospective results from the FINRISK and BiomaCaRE studies. PloS one 9, egoo63, doi:10.1371/journal.pone.0090063 (2014). 
11. Wang, T. J. et al. Prognostic utility of novel biomarkers of cardiovascular stress: the Framingham Heart Study. Circulation 126, 1596-1604, doi:10.1161/ CIRCULATIONAHA.112.129437 (2012).

12. Omland, T. et al. Impact of sex on the prognostic value of high-sensitivity cardiac troponin I in the general population: the HUNT study. Clin Chem 61, 646-656, doi:10.1373/ clinchem.2014.234369 (2015).

13. Haaf, P. et al. Risk stratification in patients with acute chest pain using three highsensitivity cardiac troponin assays. European heart journal 35, 365-375, doi:10.1093/ eurheartj/eht218 (2014).

14. Cardinaels, E. P. et al. Clinical Interpretation of Elevated Concentrations of Cardiac Troponin T, but Not Troponin I, in Nursing Home Residents. J Am Med Dir Assoc 16, 884891, doi:10.1016/j.jamda.2015.06.026 (2015).

15. Sze, J., Mooney, J., Barzi, F., Hillis, G. S. \& Chow, C. K. Cardiac Troponin and its Relationship to Cardiovascular Outcomes in Community Populations - A Systematic Review and Metaanalysis. Heart, lung \& circulation 25, 217-228, doi:10.1016/j.hlc.2015.09.001 (2016).

16. Thorsteinsdottir, I. et al. High-Sensitivity Cardiac Troponin I Is a Strong Predictor of Cardiovascular Events and Mortality in the AGES-Reykjavik Community-Based Cohort of Older Individuals. Clin Chem 62, 623-630, doi:10.1373/clinchem.2015.250811 (2016).

17. Blankenberg, S. et al. Troponin I and cardiovascular risk prediction in the general population: the BiomarCaRE consortium. European heart journal 37, 2428-2437, doi:10.1093/ eurheartj/ehw172 (2016).

18. Rubini Gimenez, M. et al. Direct comparison of high-sensitivity-cardiac troponin I vs. $\mathrm{T}$ for the early diagnosis of acute myocardial infarction. European heart journal 35, 2303-2311, doi:10.1093/eurheartj/ehu188 (2014).

19. Omland, T. et al. Prognostic value of cardiac troponin I measured with a highly sensitive assay in patients with stable coronary artery disease. Journal of the American College of Cardiology 61, 1240-1249, doi:10.1016/j.jacc.2012.12.026 (2013).

20. Hijazi, Z. et al. Comparison of cardiac troponins I and T measured with high-sensitivity methods for evaluation of prognosis in atrial fibrillation: an ARISTOTLE substudy. Clin Chem 61, 368-378, doi:10.1373/clinchem.2014.226936 (2015).

21. deFilippi, C. et al. Interpreting cardiac troponin results from high-sensitivity assays in chronic kidney disease without acute coronary syndrome. Clin Chem 58, 1342-1351, doi:10.1373/clinchem.2012.185322 (2012).

22. Zhang, Z. M., Prineas, R. J. \& Eaton, C. B. Evaluation and comparison of the Minnesota Code and Novacode for electrocardiographic Q-ST wave abnormalities for the independent prediction of incident coronary heart disease and total mortality (from the Women's Health Initiative). The American journal of cardiology 106, 18-25 e12, doi:10.1016/j. amjcard.2010.02.007 (2010).

23. Schram, M. T. et al. The Maastricht Study: an extensive phenotyping study on determinants of type 2 diabetes, its complications and its comorbidities. European journal of epidemiology 29, 439-451, doi:10.1007/s10654-014-9889-o (2014).

24. World Medical, A. World Medical Association Declaration of Helsinki: ethical principles for medical research involving human subjects. Jama 310, 2191-2194, doi:10.1001/ jama.2013.281053 (2013). 
25. Leng, G. C. \& Fowkes, F. G. The Edinburgh Claudication Questionnaire: an improved version of the WHO/Rose Questionnaire for use in epidemiological surveys. Journal of clinical epidemiology 45, 1101-1109 (1992).

26. Prineas, R. J., Crow, R. S. \& Zhang, Z. M. (Springer-Verlag London, 2010).

27. Kors, J. A. et al. Validation of a new computer program for Minnesota coding. Journal of electrocardiology 29 Suppl, 83-88 (1996).

28. Kors, J. A., Crow, R. S., Hannan, P. J., Rautaharju, P. M. \& Folsom, A. R. Comparison of computer-assigned Minnesota Codes with the visual standard method for new coronary heart disease events. American journal of epidemiology 151, 790-797 (2000).

29. Keen, H., Morrish, N. \& Lee, E. T. An analysis of serial Minnesota ECG code changes in the London cohort of the WHO Multinational Study of Vascular Disease in Diabetes. Diabetologia 44 Suppl 2, S72-77 (2001).

3o. Koerbin, G., Tate, J. R. \& Hickman, P. E. Analytical characteristics of the Roche highly sensitive troponin $\mathrm{T}$ assay and its application to a cardio-healthy population. Ann Clin Biochem 47, 524-528, doi:10.1258/acb.2010.010033 (2010).

31. Inker, L. A. et al. Estimating glomerular filtration rate from serum creatinine and cystatin C. The New England journal of medicine 367, 20-29, doi:10.1056/NEJMoa1114248 (2012).

32. Spauwen, P. J. et al. Both Low and High 24-Hour Diastolic Blood Pressure Are Associated With Worse Cognitive Performance in Type 2 Diabetes: The Maastricht Study. Diabetes care 38, 1473-148o, doi:10.2337/dc14-2502 (2015).

33. Martens, R. J. et al. Estimated GFR, Albuminuria, and Cognitive Performance: The Maastricht Study. American journal of kidney diseases : the official journal of the National Kidney Foundation 69, 179-191, doi:10.1053/j.ajkd.2016.04.017 (2017).

34. Lippi, G. \& Cervellin, G. High-sensitivity troponin $\mathrm{T}$ is more susceptible than highsensitivity troponin I to impaired renal function. The American journal of cardiology 112, 1985, doi:10.1016/j.amjcard.2013.10.003 (2013).

35. Bjurman, C. et al. High-sensitive cardiac troponin, NT-proBNP, hFABP and copeptin levels in relation to glomerular filtration rates and a medical record of cardiovascular disease. Clinical biochemistry 48, 302-307, doi:10.1016/j.clinbiochem.2015.01.008 (2015).

36. Martens, R. J. et al. Estimated Glomerular Filtration Rate and Albuminuria Are Associated with Biomarkers of Cardiac Injury in a Population-Based Cohort Study: The Maastricht Study. Clin Chem 63, 887-897, doi:10.1373/clinchem.2016.266031 (2017). 


\section{SUPPLEMENTAL DATA}

\section{Supplemental \\ Figure 5.1. \\ Study flow \\ diagram.}

Excluded, variables were not mutually exclusive $(n=208)$

- TIDM ( $n=37)$

- Other DM $(n=4)$

- self-reported history of AMI $(n=169)$
Individuals

The Maastricht Study cohort assessed for eligibility

$(n=3,451)$

Missing (in)dependent variables $(\mathrm{n}=|0|)$

- hs-cTnT / hs-cTnl levels ( $=29)$

- ECG data $(n=72)$

Missing data, variables were not mutually exclusive $(n=126)$

- eqFR $R_{\text {crovs }}(n=2)$

- office systolic blood pressure $(n=2)$

- antihypertensive medication $(n=4)$

- smoking behavior $(n=60)$

- self-reported history of AMI $(n=75)$

- waist-to-hip ratio ( $n=4)$

- level of education ( $n=72$ )

- alcohol consumption ( $=66$ )

- lipid-modifying medication ( $n=4)$ 


hs-cTnt low
hs-cTnT high
hs-cTnl low
hs-cTnl high
both hs-cTnT and hs-cTnl low
hs-cTnT high and hs-cTnl low
hs-cTnT low and hs-cTnl high
both hs-cTnT and hs-cTnl high

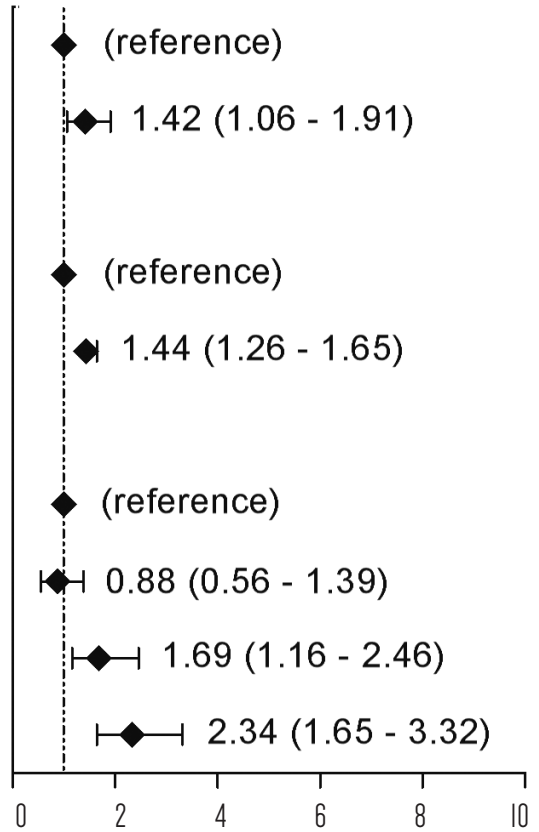

odds ratio
Supplemental

Figure 5.2.

Associations

between

combined hs-cTn

categories and

liberal definition

of ECG changes

indicative

of cardiac

abnormalities.

Associations between combined hs-cTn categories and liberal definition of ECG changes indicative of cardiac abnormalities. Definitions of "low" and "high" $h s-c T n$ categories were based on the sex-specific $75^{\text {th }}$ percentiles of hscTnI and hs-cTnT (hs-cTnI, women: $2.20 \mathrm{ng} / \mathrm{L}$; hs-cTnI, men: $3.70 \mathrm{ng} / \mathrm{L}$; hs-cTnT, women: $5.55 \mathrm{ng} / \mathrm{L} ; \mathrm{hs}-c T n T$, men: 9.36 $\mathrm{ng} / \mathrm{L}$ ). Category "low" included participants with hs-cTn levels < the sex-specific $75^{\text {th }}$ percentile and category "high" included participants $\geq$ the sex-specific $75^{\text {th }}$ percentile. Model was adjusted for sex, age, glucose metabolism status, eGFR, smoking behavior, total-to-HDL cholesterol ratio, triglyceride levels, lipid-modifying medication, office systolic blood pressure, antihypertensive medication, waist-to-hip ratio, alcohol consumption and educational level.

\begin{tabular}{|lll|}
\hline Minnesota coding & Description & N (\%) \\
\hline I.I.I - I.2.8 & major or medium abnormalities in Q / QS patterns & $78(2.6 \%)$ \\
\hline I.3.I, I.3.2 & borderline abnormalities in Q/OS patterns & $65(2.2 \%)$ \\
\hline 4.I.I. 4.I.2. 4.2, 4.3 & ST-segment abnormalities & $82(2.7 \%)$ \\
\hline 5.I, 5.2. 5.3 & abnormal T-waves & $310(10.3 \%)$ \\
\hline 7.I.I. 7.I.2 & left bundle branch block & $28(0.9 \%)$ \\
\hline
\end{tabular}

Supplemental Table 5.1. Prevalence of Minnesota coding categories of ECG abnormalities of the total study population. 


\begin{tabular}{|c|c|c|c|c|c|}
\hline \multirow{26}{*}{$\begin{array}{r}\text { Supplemental } \\
\text { Table 5.2. } \\
\text { Associations } \\
\text { of hs-cTnI and } \\
\text { hs-cTnT with } \\
\text { ECG changes } \\
\text { indicative } \\
\text { of cardiac } \\
\text { abnormalities. }\end{array}$} & & \multicolumn{2}{|c|}{$\begin{array}{c}\text { hs-cTnl } \\
\text { In, I-SD increase }\end{array}$} & \multicolumn{2}{|c|}{$\begin{array}{c}\text { hs-cTnT } \\
\text { In, I-SD increase }\end{array}$} \\
\hline & & OR (95\% CI) & p-value & OR (95\% Cl) & $p$-value \\
\hline & \multicolumn{5}{|l|}{ LoD } \\
\hline & modell & $1.52(1.33-1.73)$ & $<0.001$ & I.42 (I.23-I.65) & $<0.001$ \\
\hline & model 2 & I.51 (1.31-|.75) & $<0.001$ & 1.32 (I.|I-|.58) & 0.002 \\
\hline & model 3 & 1.54 (I.33-1.77) & $<0.001$ & $1.38(1.15-1.66)$ & 0.001 \\
\hline & model 4A & $1.52(1.31-1.76)$ & $<0.001$ & I.35 (I..I-I.63) & 0.002 \\
\hline & model 4B & I.61 (1.38-1.89) & $<0.001$ & $1.35(1.10-1.65)$ & 0.004 \\
\hline & \multicolumn{5}{|l|}{$20 \%$ CV } \\
\hline & modell & $1.56(1.36-1.80)$ & $<0.001$ & I.33 (I.17-I.52) & $<0.001$ \\
\hline & model 2 & $1.56(1.33-1.82)$ & $<0.001$ & I.23 (I.06-I.43) & 0.007 \\
\hline & model 3 & $1.58(1.35-1.85)$ & $<0.001$ & $1.27(1.08-1.48)$ & 0.003 \\
\hline & model 4A & 1.56 (I.32-1.83) & $<0.001$ & 1.25 (I.06-I.47) & 0.007 \\
\hline & model 4B & 1.67 (I.41-1.98) & $<0.001$ & $1.23(1.04-\mid .47)$ & 0.019 \\
\hline & \multicolumn{5}{|l|}{$10 \%$ CV } \\
\hline & modell & $1.29(1.17-1.43)$ & $<0.001$ & $1.12(0.99-1.27)$ & 0.069 \\
\hline & model 2 & 1.30 (I.16-I.44) & $<0.001$ & 1.06 (0.93-1.22) & 0.386 \\
\hline & model 3 & $1.30(1.17-1.45)$ & $<0.001$ & $1.07(0.93-1.23)$ & 0.325 \\
\hline & model 4A & $1.30(1.17-1.46)$ & $<0.001$ & $1.07(0.93-1.23)$ & 0.344 \\
\hline & model 4B & $1.34(1.20-1.50)$ & $<0.001$ & $1.06(0.92-1.23)$ & 0.412 \\
\hline & \multicolumn{5}{|l|}{ LoO } \\
\hline & modell & $1.24(1.13-1.37)$ & $<0.001$ & $1.12(0.99-1.27)$ & 0.069 \\
\hline & model 2 & I.25 (I.I3-I.38) & $<0.001$ & 1.06 (0.93-1.22) & 0.386 \\
\hline & model 3 & $1.26(1.14-1.39)$ & $<0.001$ & $1.07(0.93-1.23)$ & 0.325 \\
\hline & model 4A & 1.26 (I.I3-1.39) & $<0.001$ & 1.07 (0.93-1.23) & 0.344 \\
\hline & model 4B & 1.29 (1.16-1..43) & $<0.001$ & $1.06(0.92-1.23)$ & 0.412 \\
\hline
\end{tabular}

Hs-cTn values below the lower limits of measurement were set equal to the LoD, $20 \% \mathrm{CV}, 10 \% \mathrm{CV}$ and LoQ, respectively. Hs-cTnI, LoD: 1.7 ng/L, 20\% CV: 1.3 ng/L, 10\% CV: 4.7 ng/L, LoQ: 6.7 ng/L; hs-cTnT, LoD: $5 \mathrm{ng} / \mathrm{L}, 20 \%$ CV: $6.8 \mathrm{ng} / \mathrm{L}$, 10\% CV: $13 \mathrm{ng} / \mathrm{L}, \mathrm{LoQ}: 13 \mathrm{ng} / \mathrm{L}$. For hs-cTnI a LoD value of $1.7 \mathrm{ng} / \mathrm{L}$ and LoQ value of $6.7 \mathrm{ng} / \mathrm{L}$ was chosen to obtain similar proportions for troponin I and T of participants with values above the LoD and LoQ, respectively. Model 1: crude model; model 2: model $1+$ sex, age, glucose metabolism status; model 3: model $2+e G F R ;$ model 4A: model 3 + smoking behavior, total-to-HDL cholesterol ratio, triglyceride levels, lipid-modifying medication, office systolic blood pressure, antihypertensive medication, waist-to-hip ratio, alcohol consumption, educational level; model $4 \mathrm{~B}$ : model $4 \mathrm{~A}$ with replacement of office systolic blood pressure by 24 h average ambulatory systolic blood pressure. 


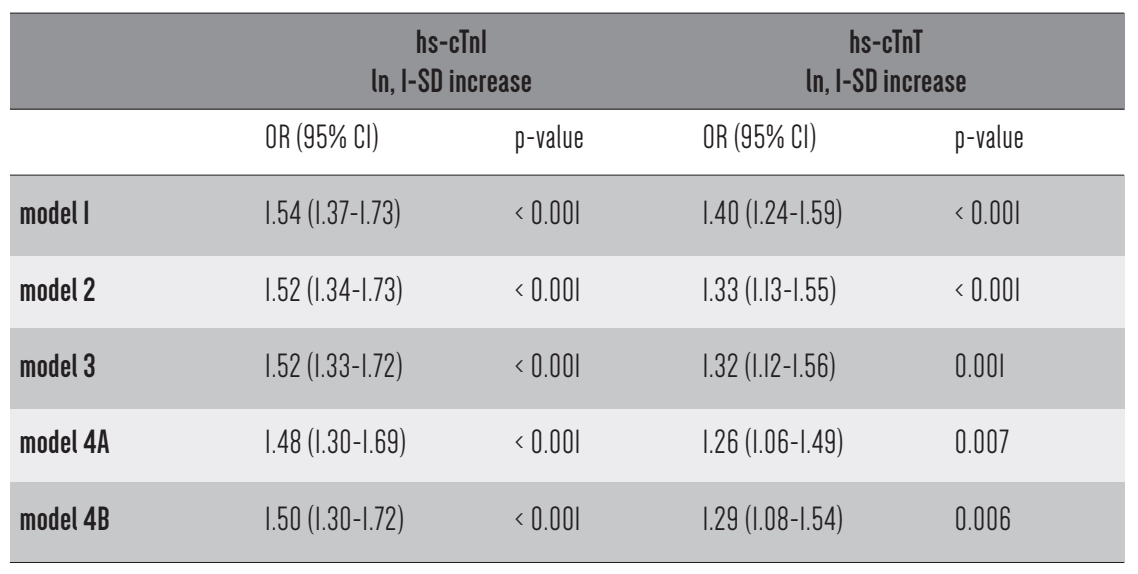

Supplemental

Table 5.3.

Associations

of hs-cTnI and hs-cTnT with

liberal definition

of ECG changes

indicative

of cardiac

abnormalities.

Model 1: crude model; model 2: model $1+$ sex, age, glucose metabolism status; model 3: model $2+$ eGFR; model 4A: model 3 + smoking behavior, total-to-HDL cholesterol ratio, triglyceride levels, lipid-modifying medication, office systolic blood pressure, antihypertensive medication, waist-to-hip ratio, alcohol consumption, educational level; model 4B: model $4 \mathrm{~A}$ with replacement of office systolic blood pressure by $24 \mathrm{~h}$ average ambulatory systolic blood pressure. 


\begin{tabular}{|c|c|c|c|c|c|}
\hline $\begin{array}{r}\text { Supplemental } \\
\text { Table 5.4. } \\
\text { Clinical } \\
\text { characteristics } \\
\text { stratified by } \\
\text { hs-cTnI categories } \\
\text { based on the } \\
\text { sex-specific } 75 \text { th } \\
\text { percentiles and } \\
\text { sex-specific } 99^{\text {th }} \\
\text { percentiles. }\end{array}$ & variable & $\begin{array}{l}\text { hs-cTnl } \\
\text { < sex-specific } \\
75^{\text {th }} \text { percentiles } \\
\text { ( } \mathrm{n}=2278 \text { ) }\end{array}$ & $\begin{array}{l}\text { hs-cTnl } \\
\text { əsex-specific } \\
7^{\text {th }} \text { percentiles } \\
\text { ( }=738 \text { ) }\end{array}$ & $\begin{array}{l}\text { hs-cTnl } \\
\text { s sex-specific } \\
99^{\text {th }} \text { percentiles } \\
(\mathrm{n}=2987)\end{array}$ & $\begin{array}{l}\text { hs-cTnl } \\
\geq \text { sex-specific } \\
\text { 99th percentiles } \\
(\mathrm{n}=29)\end{array}$ \\
\hline \multirow{16}{*}{$\begin{array}{l}\text { hs-cTnI categories } \\
\text { based on the } \\
\text { sex-specific } 75 \text { th } \\
\text { percentiles and } \\
\text { sex-specific } 99^{\text {th }} \\
\text { percentiles. }\end{array}$} & age (years) & $59(8)$ & $63(7)$ & $60(8)$ & $62(9)$ \\
\hline & $\operatorname{sex}(m a l e)$ & $\| 5 \mid(50.5 \%)$ & $375(50.8 \%)$ & $|5| \mid(50.6 \%)$ & $15(51.7 \%)$ \\
\hline & $\operatorname{BMI}\left(\mathrm{kg} / \mathrm{m}^{2}\right)$ & $26.5(4.3)$ & $28.0(4.9)$ & $26.9(4.5)$ & $26.0(3.7)$ \\
\hline & eGFR & & & & \\
\hline & $<60 \mathrm{~mL} / \mathrm{min} / \mathrm{l} .73 \mathrm{~m}^{2}$ & $48(2.1 \%)$ & $61(8.3 \%)$ & $106(3.5 \%)$ & $3(10.3 \%)$ \\
\hline & $60->90 \mathrm{~mL} / \mathrm{min} / 1.73 \mathrm{~m}^{2}$ & I022 (44.9\%) & $4 \mid 6(56.4 \%)$ & $14 \mid 9(47.5 \%)$ & $19(65.5 \%)$ \\
\hline & $\geq 90 \mathrm{~mL} / \mathrm{min} / \mathrm{l} .73 \mathrm{~m}^{2}$ & I208 (53.0\%) & $26 \mathrm{I}(35.4 \%)$ & 1462 (48.9\%) & $7(24.1 \%)$ \\
\hline & glucose metabolism & & & & \\
\hline & NGM & |394 (6l.2\%) & $382(51.8 \%)$ & $1759(58.9 \%)$ & $17(58.6 \%)$ \\
\hline & IFG & $105(4.6 \%)$ & $24(3.3 \%)$ & $129(4.3 \%)$ & $0(0 \%)$ \\
\hline & IGT & $237(10.4 \%)$ & $93(12.6 \%)$ & $326(10.9 \%)$ & $4(13.8 \%)$ \\
\hline & T2DM & $542(23.8 \%)$ & $239(32.4 \%)$ & $773(25.9 \%)$ & $8(27.6 \%)$ \\
\hline & office BP, systolic (mmHg) & |33 (17) & $140(20)$ & $135(18)$ & $142(8)$ \\
\hline & office BP, diastolic (mmHg) & $76(10)$ & $77(I I)$ & $76(10)$ & $77(\|)$ \\
\hline & hypertension (yes) & $1098(48.2 \%)$ & $530(71.8 \%)$ & $\operatorname{l609}(53.9 \%)$ & $19(65.5 \%)$ \\
\hline & antihypertensive medication (yes) & $717(31.5 \%)$ & $391(53.0 \%)$ & $1095(36.7 \%)$ & I3 (44.8\%) \\
\hline \multirow{9}{*}{$\begin{array}{r}\text { Sex-specific } \\
75^{\text {th }} \text { percentiles } \\
\text { of hs-cTnI: } \\
\text { women, } 2.20 \\
n g / l ; \text { men, } 3.70 \\
n g / / \text { Sex-specific } \\
\text { ng } 9^{\text {th }} \text { percentiles } \\
\text { ofhs-cTnI: } \\
\text { women, } 13.15 \\
\text { ng/l; men, 25.56 } \\
\text { ng/l. Continuous } \\
\text { variables are } \\
\text { expressed as } \\
\text { mean (SD) or } \\
\text { median (IQR) } \\
\text { depending on } \\
\text { their distribution. } \\
\text { Categorical data } \\
\text { are reported } \\
\text { as } n(\%) .\end{array}$} & smoking behavior & & & & \\
\hline & never & $813(35.7 \%)$ & $277(37.5 \%)$ & $1078(36.1 \%)$ & $12(41.4 \%)$ \\
\hline & former & $\| 5 \mid(50.5 \%)$ & $374(50.7 \%)$ & $1508(50.5 \%)$ & $17(58.6 \%)$ \\
\hline & current & $3 \mid 4(\mid 3.8 \%)$ & $87(11.8 \%)$ & $401(13.4 \%)$ & $0(0 \%)$ \\
\hline & total-to-HDL cholesterol ratio & $3.4(2.8-4.3)$ & $3.6(2.8-4.3)$ & $3.5(2.8-4.3)$ & $3.2(2.8-4.3)$ \\
\hline & triglycerides (mmol/L) & $1.2(0.9-1.7)$ & $1.3(0.9-1.8)$ & $1.2(0.9-1.7)$ & $1.0(0.9-1.4)$ \\
\hline & lipid-modifying medication (yes) & $682(29.9 \%)$ & $310(42.0 \%)$ & $984(32.9 \%)$ & $8(27.6 \%)$ \\
\hline & hs-cInl (ng/L) & $1.5(1.1-2.1)$ & $4.3(3.1-6.6)$ & $1.9(1.2-2.9)$ & $27.9(20.3-57.0)$ \\
\hline & hs-cTnT (ng/L) & $4.8(3.5-6.7)$ & $7.8(5.5-11.1)$ & $5.3(3.7-7.7)$ & $11.7(6.4-17.2)$ \\
\hline
\end{tabular}




\begin{tabular}{|c|c|c|c|c|c|}
\hline variable & $\begin{array}{l}\text { hs-cTnT } \\
\text { < sex-specific } \\
75^{\text {th }} \text { percentiles } \\
\text { ( } \mathrm{n}=2266 \text { ) }\end{array}$ & $\begin{array}{l}\text { hs-cTnT } \\
\text { ə sex-specific } \\
75^{\text {th }} \text { percentiles } \\
\text { ( }=750)\end{array}$ & $\begin{array}{l}\text { hs-cTnT } \\
\text { s sex-specific } \\
99^{\text {th }} \text { percentiles } \\
(\mathrm{n}=2987)\end{array}$ & $\begin{array}{l}\text { hs-cTnT } \\
\text { ”s sex-specific } \\
99^{\text {th }} \text { percentiles } \\
(\mathrm{n}=29)\end{array}$ & \multirow{4}{*}{$\begin{array}{l}\text { Supplemental } \\
\text { Table 5.5. } \\
\text { Clinical } \\
\text { characteristics } \\
\text { stratified by hs- } \\
\text { cTnT categories } \\
\text { based on the } \\
\text { sex-specific } 75^{\text {th }} \\
\text { percentiles and } \\
\text { sex-specific } 99^{\text {th }} \\
\text { percentiles. }\end{array}$} \\
\hline age (years) & $58(8)$ & $64(7)$ & $60(8)$ & $66(7)$ & \\
\hline sex (male) & $\| 47(50.6 \%)$ & $379(50.5 \%)$ & $|5| \mid(50.6 \%)$ & $15(51.7 \%)$ & \\
\hline $\operatorname{BMI}\left(\mathrm{kg} / \mathrm{m}^{2}\right)$ & $26.5(4.2)$ & $28.2(5.1)$ & $26.9(4.5)$ & $27.9(6.1)$ & \\
\hline \multicolumn{6}{|l|}{ eGFR } \\
\hline$<60 \mathrm{~mL} / \mathrm{min} / 1.73 \mathrm{~m}^{2}$ & $27(1.2 \%)$ & $82(10.9 \%)$ & $98(3.3 \%)$ & $\|(37.9 \%)$ & \\
\hline 60 - > $90 \mathrm{~mL} / \mathrm{min} / .73 \mathrm{~m}^{2}$ & $958(42.3 \%)$ & $480(64.0 \%)$ & $1420(47.5 \%)$ & $18(62.1 \%)$ & \\
\hline$=90 \mathrm{~mL} / \mathrm{min} / \mathrm{l} .73 \mathrm{~m}^{2}$ & $|28|(56.5 \%)$ & $188(25.1 \%)$ & $1469(49.2 \%)$ & $0(0 \%)$ & \\
\hline \multicolumn{6}{|l|}{ glucose metabolism } \\
\hline NGM & $1467(64.7 \%)$ & $309(41.2 \%)$ & $1769(59.2 \%)$ & $7(24.1 \%)$ & \\
\hline IFG & $97(4.3 \%)$ & $32(4.3 \%)$ & $129(4.3 \%)$ & $0(0 \%)$ & \\
\hline IGT & $239(10.5 \%)$ & 9I (I2.1\%) & $328(\| 1.0 \%)$ & $2(6.9 \%)$ & \\
\hline T2DM & $463(20.4 \%)$ & $318(42.4 \%)$ & $761(25.5 \%)$ & $20(69.0 \%)$ & \\
\hline office BP, systolic (mmHg) & |33 (17) & $139(19)$ & $135(18)$ & $140(23)$ & \\
\hline office BP, diastolic (mmHg) & $76(10)$ & $76(10)$ & $76(10)$ & $72(11)$ & \\
\hline hypertension (yes) & $1082(47.7 \%)$ & $546(72.8 \%)$ & $1604(53.7 \%)$ & $24(82.8 \%)$ & \\
\hline antihypertensive medication (yes) & $670(29.6 \%)$ & $438(58.4 \%)$ & $1087(36.4 \%)$ & $21(72.4 \%)$ & \\
\hline smoking behavior & & & & & \multirow{9}{*}{$\begin{array}{l}\text { Sex-specific } \\
75^{\text {th percentiles }} \\
\text { ofhs-cTnT: } \\
\text { women, } 5.55 \\
\text { ng/l/; men, } 9.36 \\
\text { ngll. Sex-specific } \\
99^{\text {th }} \text { percentiles } \\
\text { ofhs-cTnT: } \\
\text { women, } 16.28 \\
\text { ng/li;men, } 29.50 \\
\text { ng/l. Continuous } \\
\text { variables are } \\
\text { expressed as } \\
\text { mean (SD) or } \\
\text { median (IQR) } \\
\text { depending on } \\
\text { their distribution. } \\
\text { Categorical data } \\
\text { are reported } \\
\text { as } n(\%) \text {. }\end{array}$} \\
\hline never & $821(36.2 \%)$ & $269(35.9 \%)$ & 1081 (36.2\%) & $9(31.0 \%)$ & \\
\hline former & ॥|33 (50.0\%) & $392(52.3 \%)$ & $1507(50.5 \%)$ & $18(62.1 \%)$ & \\
\hline current & $312(13.8 \%)$ & $89(\mid 1.9 \%)$ & $399(\mid 3.4 \%)$ & $2(6.9 \%)$ & \\
\hline total-to-HDL cholesterol ratio & $3.5(2.8-4.3)$ & $3.5(2.8-4.3)$ & $3.5(2.8-4.3)$ & $3.7(2.7-4.3)$ & \\
\hline triglycerides (mmol/L) & $1.2(0.9-1.6)$ & $1.3(1.0-1.8)$ & $1.2(0.9-1.7)$ & $1.4(1.0-2.5)$ & \\
\hline lipid-modifying medication (yes) & $615(27.1 \%)$ & $377(50.3 \%)$ & $980(32.8 \%)$ & $12(41.4 \%)$ & \\
\hline hs-cTnl (ng/L) & I.6 (I..--2.4) & $3.0(1.9-5.1)$ & $1.9(1.2-2.9)$ & $4.6(3 .|-|-\mid 6.0)$ & \\
\hline hs-cTnT (ng/L) & $4.5(3.5-5.9)$ & $10.1(7.1-12.9)$ & $5.3(3.7-7.6)$ & $33.9(29.5-50.5)$ & \\
\hline
\end{tabular}




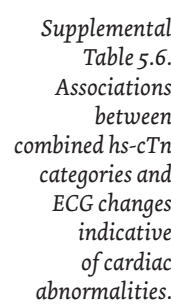

\begin{tabular}{lllll}
\hline threshold & hs-cTn categories & n & OR $(95 \%$ CI $)$ & p-value \\
\hline $66^{\text {th }}$ percentile & both hs-cTnT and hs-cTnl low & 1526 & reference & \\
& hs-cTnT high and hs-cTnl low & 381 & $0.98(0.43-2.22)$ & 0.953 \\
& hs-cTnT low and hs-cTnl high & 444 & $1.67(0.86-3.25)$ & 0.129 \\
& both hs-cTnT and hs-cTnl high & 625 & $3.32(1.91-5.77)$ & $<0.001$
\end{tabular}

$70^{\text {th }}$ percentile both hs-cTnT and hs-cTnl low

1692 reference

hs-cTnT high and hs-cTnl low

$360 \quad 1.02(0.46-2.30)$

0.955

hs-cTnT low and hs-cTnl high

418

$1.74(0.90-3.34)$

0.098

both hs-cTnT and hs-cTnl high

546

$3.57(2.07-6.16)$

$<0.001$

$80^{\text {th }}$ percentile both hs-cTnT and hs-cTnl low

2093 reference

hs-cTnT high and hs-cTnl low

29

$1.24(0.58-2.68)$

0.577

hs-cTnT low and hs-cTnl high

320

$2.35(1.27-4.34)$

0.006

both hs-cTnT and hs-cTnl high

370

$3.92(2.20-6.97)$

$<0.001$

90 percentile both hs-cTnT and hs-cTnl low
hs-cTnT high and hs-cTnl low
hs-cTnT low and hs-cTnl high
both hs-cTnT and hs-cTnl high

2524 reference

$182 \quad 1.34(0.60-2.96) \quad 0.477$

I9 $\quad 2.53(1.34-4.80) \quad 0.004$

$\| 9 \quad 3.83(1.90-7.72) \quad<0.001$

Definitions of "low" and "high" hs-cTn categories were based on sex-specific $66^{\text {th }}$ percentiles of hs-cTnI and hs-cTnT (hscTnI, women: $1.80 \mathrm{ng} / \mathrm{L}$; hs-cTnI, men: $3.10 \mathrm{ng} / \mathrm{L} ; \mathrm{hs}-c T n T$, women: $4.95 \mathrm{ng} / \mathrm{L} ; \mathrm{hs}-c T n T$, men: $8.34 \mathrm{ng} / \mathrm{L}$ ), $70^{\text {th }}$ percentiles (hs-cTnI, women: $1.90 \mathrm{ng} / \mathrm{L}$; hs-cTnI, men: $3.30 \mathrm{ng} / \mathrm{L} ; \mathrm{hs}$-cTnT, women: $5.18 \mathrm{ng} / \mathrm{L} ; \mathrm{hs}$-cTnT, men: $8.68 \mathrm{ng} / \mathrm{L}$ ), $80^{\text {th }}$ percentiles (hs-cTnI, women: $2.40 \mathrm{ng} / \mathrm{L}$; hs-cTnI, men: $4.00 \mathrm{ng} / \mathrm{L}$; $\mathrm{hs}$-cTnT, women: $5.93 \mathrm{ng} / \mathrm{L} ; \mathrm{hs}-\mathrm{cTnT}$, men: 10.17 $\mathrm{ng} / \mathrm{L}$ ) and $9 \mathrm{o}^{\text {th }}$ percentiles (hs-cTnT, women: $7.68 \mathrm{ng} / \mathrm{L} ; \mathrm{hs}-\mathrm{cTnI}$, women: $3.50 \mathrm{ng} / \mathrm{L} ; \mathrm{hs}$-cTnI, men: $6.30 \mathrm{ng} / \mathrm{L} ; \mathrm{hs}$-cTnT, men: $12.83 \mathrm{ng} / \mathrm{L}$ ). Category "low" included participants with hs-cTn levels $<$ the respective percentile and category "high" included participants $\geq$ the respective percentile. Model was adjusted for sex, age, glucose metabolism status, eGFR, smoking behavior, total-to-HDL cholesterol ratio, triglyceride levels, lipid-modifying medication, office systolic blood pressure, antihypertensive medication, waist-to-hip ratio, alcohol consumption and educational level. 


$\begin{array}{lllll} & \text { hs-cTnT } & \text { hs-cTnT high } & \text { hs-cTnT low } & \text { hs-cTnT } \\ \text { and } & \text { and } & \text { and } & \text { and } \\ \text { hs-cTnl low } & \text { hs-cTnl low } & \text { hs-cTnl high } & \text { hs-cTnl high } \\ (n=1934) & (n=344) & (n=332) & (n=406) \\ \text { variable } & & & \end{array}$

\begin{tabular}{lllll}
\hline age (years) & $58(8)$ & $63(7)$ & $60(8)$ & $65(7)$ \\
\hline sex (male) & $979(50.6 \%)$ & $172(50.0 \%)$ & $168(50.6 \%)$ & $207(51.0 \%)$ \\
BMI $\left(\mathrm{kg} / \mathrm{m}^{2}\right)$ & $26.3(4 . \mathrm{l})$ & $27.7(4.8)$ & $27.3(4.4)$ & $28.7(5.3)$ \\
\hline
\end{tabular}

Supplemental Table 5.7.

Clinical characteristics stratified by combined hs-cTn categories based on the sex-specific $75^{\text {th }}$ percentiles.

eGFR

$\begin{array}{lllll}<60 \mathrm{~mL} / \mathrm{min} / 1.73 \mathrm{~m}^{2} & 22(1.1 \%) & 26(7.6 \%) & 5(1.5 \%) & 56(13.8 \%) \\ 60->90 \mathrm{~mL} / \mathrm{min} / 1.73 \mathrm{~m}^{2} & 809(41.8 \%) & 213(61.9 \%) & 149(44.9 \%) & 267(65.8 \%) \\ \geq 90 \mathrm{~mL} / \mathrm{min} / 1.73 \mathrm{~m}^{2} & 1103(57.0 \%) & 105(30.5 \%) & 178(53.6 \%) & 83(20.4 \%)\end{array}$

\section{glucose metabolism}

\begin{tabular}{|c|c|c|c|c|c|}
\hline NGM & I253 (64.8\%) & $|4|(41.0 \%)$ & $214(64.5 \%)$ & $168(41.4 \%)$ & \multirow{17}{*}{ 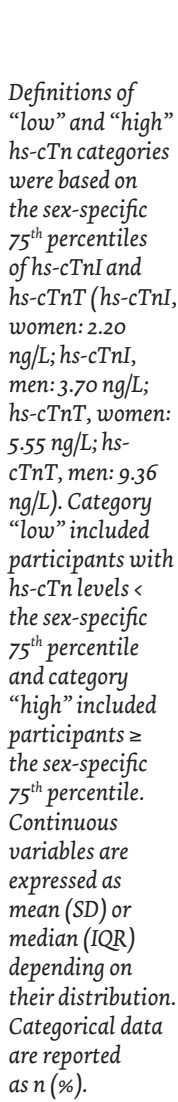 } \\
\hline IFG & $90(4.7 \%)$ & $15(4.4 \%)$ & $7(2.1 \%)$ & $17(4.2 \%)$ & \\
\hline IGT & $196(10.1 \%)$ & $4 \mid(11.9 \%)$ & $43(13.0 \%)$ & $50(12.3 \%)$ & \\
\hline T2DM & $395(20.4 \%)$ & $147(42.7 \%)$ & $68(20.5 \%)$ & $|7|(42.1 \%)$ & \\
\hline office BP, systolic (mmHg) & $132(17)$ & $136(17)$ & $139(\mid 9)$ & $142(20)$ & \\
\hline office BP, diastolic (mmHg) & $76(10)$ & $76(10)$ & $78(11)$ & $76(11)$ & \\
\hline hypertension (yes) & $870(45.0 \%)$ & $228(66.3 \%)$ & $212(63.9 \%)$ & $318(78.3 \%)$ & \\
\hline antihypertensive medication (yes) & $531(27.5 \%)$ & $186(54.1 \%)$ & $139(41.9 \%)$ & $252(62.1 \%)$ & \\
\hline smoking behavior & & & & & \\
\hline never & $704(36.4 \%)$ & $\log (31.7 \%)$ & $117(35.2 \%)$ & $160(39.4 \%)$ & \\
\hline former & $959(49.6 \%)$ & $192(55.8 \%)$ & $174(52.4 \%)$ & $200(49.3 \%)$ & \\
\hline current & $271(14.0 \%)$ & $43(12.5 \%)$ & $41(12.3 \%)$ & $46(11.3 \%)$ & \\
\hline total-to-HDL cholesterol ratio & $3.4(2.8-4.3)$ & $3.4(2.8-4.3)$ & $3.6(3.0-4.3)$ & $3.5(2.8-4.3)$ & \\
\hline triglycerides (mmol/L) & $1.2(0.9-1.6)$ & $1.3(1.0-1.8)$ & $1.2(0.9-1.7)$ & $1.3(1.0-1.9)$ & \\
\hline lipid-modifying medication (yes) & $5 \mathrm{II}(26.4 \%)$ & $17 \mid(49.7 \%)$ & $104(31.3 \%)$ & $206(50.7 \%)$ & \\
\hline hs-cTnl (ng/L) & $1.5(1.1-2.0)$ & $1.9(1.4-2.5)$ & $4.0(2.9-5.5)$ & $4.7(3.5-7.4)$ & \\
\hline hs-cTnT (ng/L) & $4.3(3.2-5.7)$ & $9.8(6.6-11.9)$ & $5.3(4.3-7.4)$ & $10.6(7.8-14.0)$ & \\
\hline
\end{tabular}




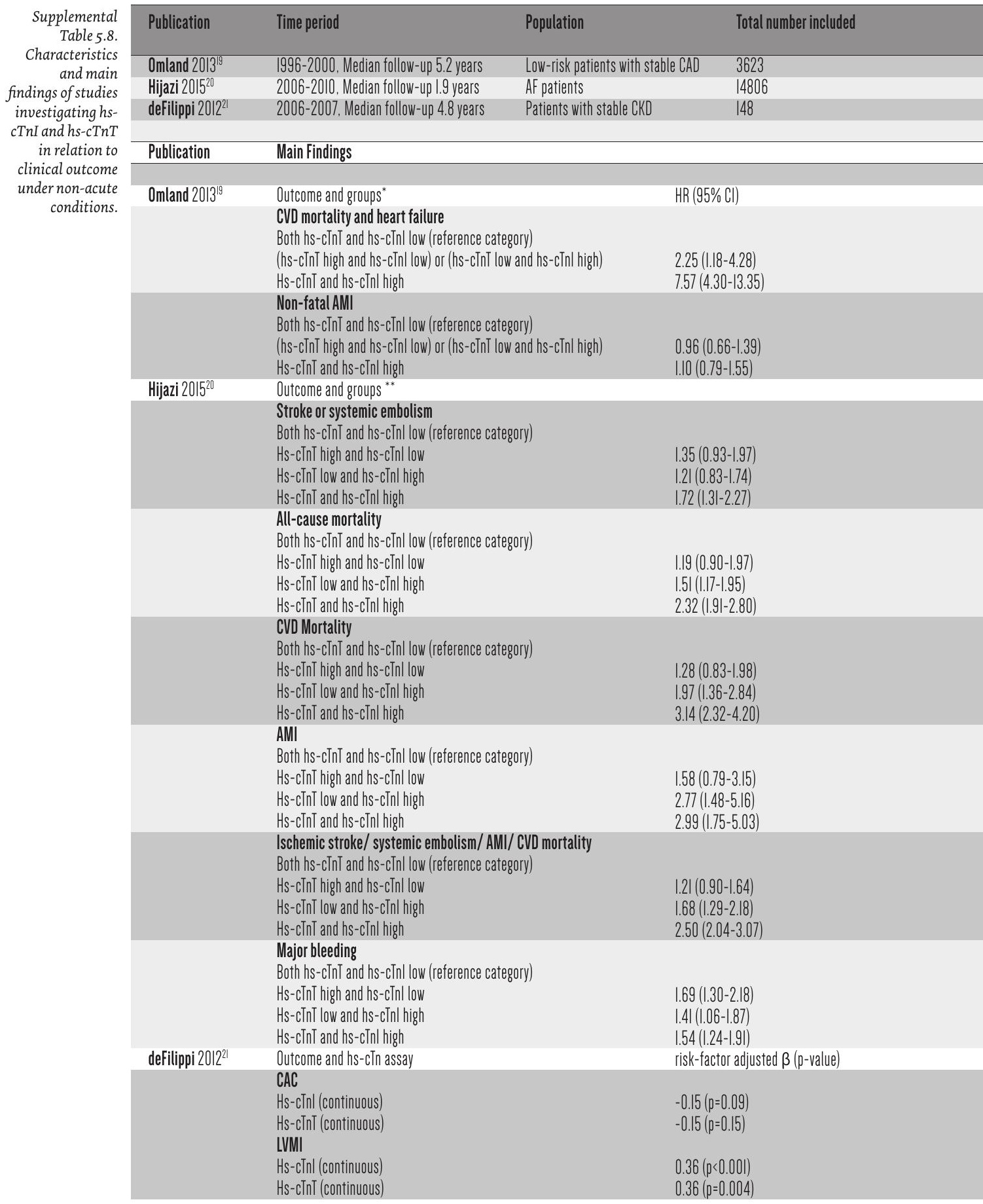




\begin{tabular}{lll} 
Sex (male) & Correlation or concordance hs-cTnl/hs-cTnT & Outcome \\
\hline $2933(81.0 \%)$ & $r=0.44$ & CVD mortality or heart failure, nonfatal AMl \\
$9517(64.3 \%)$ & $r=0.70$ & Stroke or systemic embolism, all-cause mortality, CVD mortality, AMI, major bleeding \\
$102(68.9 \%)$ & $\mathrm{k}=0.40$ & CAC, LVMI
\end{tabular}

* Definitions of "low" and "high" hs-cTn categories were based on the sex-specific $75^{\text {th }}$ percentiles of hscTnI and hs-cTnT (hs-cTnI, women: $2.8 \mathrm{ng} / \mathrm{L} ; \mathrm{hs}-\mathrm{cTnI}$, men: $4.2 \mathrm{ng} / \mathrm{L}$; hs-cTnT, women: $3.9 \mathrm{ng} / \mathrm{L} ; \mathrm{hs}-\mathrm{cTnT}$, men: $5.6 \mathrm{ng} / \mathrm{L}$ ). ${ }^{\star *}$ Definitions of "low" and "high" hs-cTn categories were based on the median of hs-cTnI and hs-cTnT

(hs-cTnI: 6.3 ng/L; hs-cTnT;

$17.0 \mathrm{ng} / \mathrm{L}$ ). 


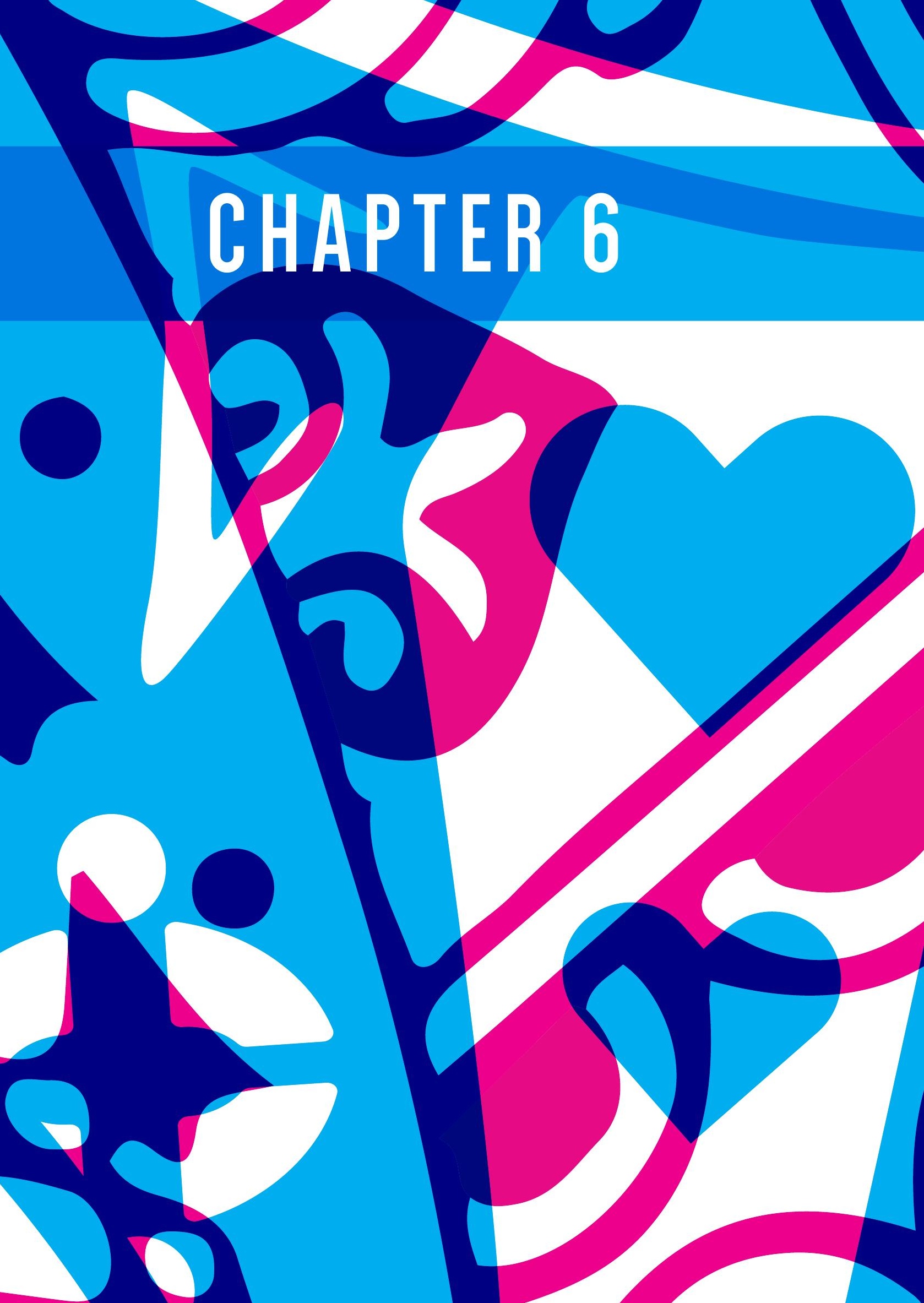




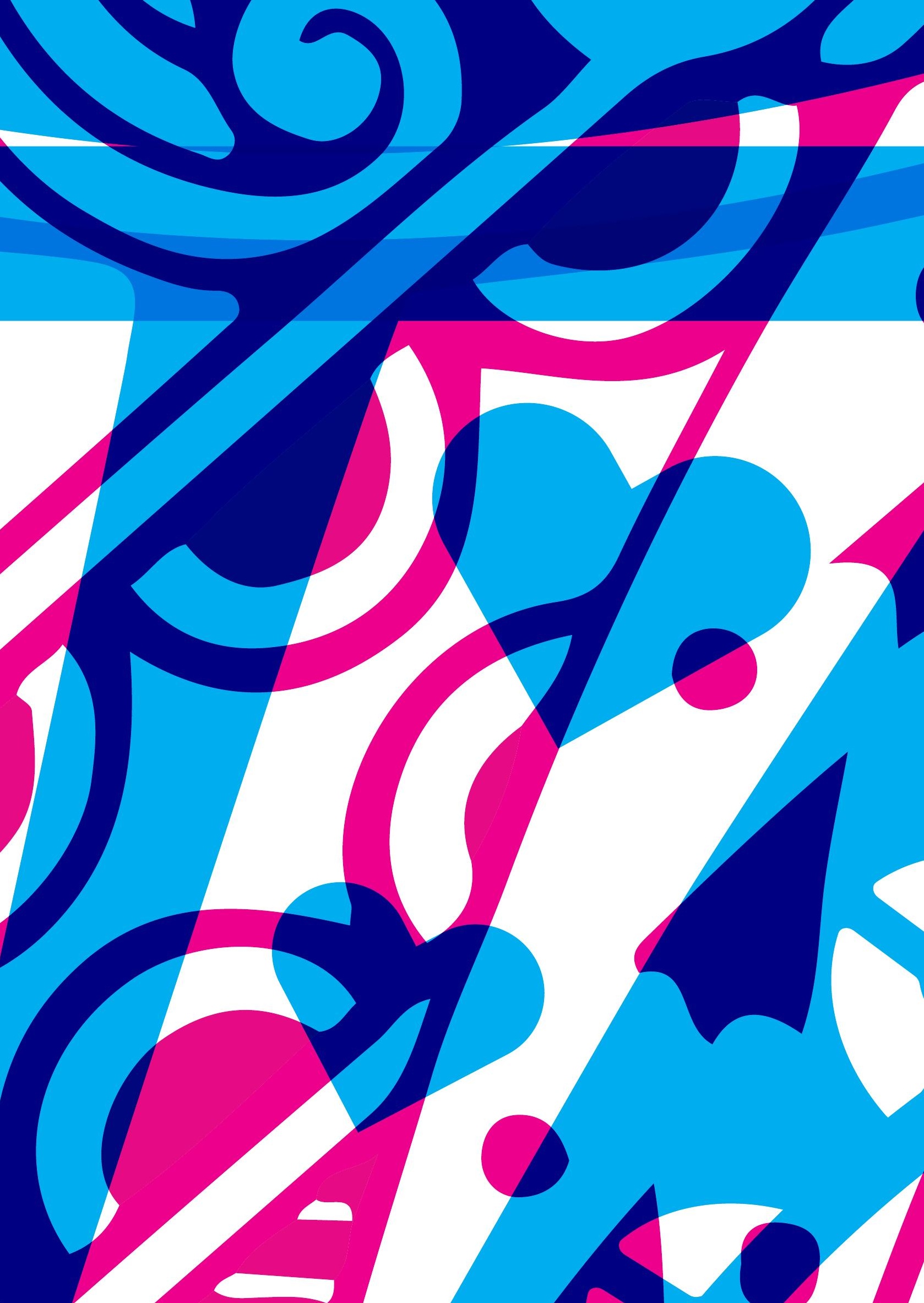




\section{CHAPTER 6}

\section{ESTIMATED GLOMERULAR \\ FILTRATION RATE AND}

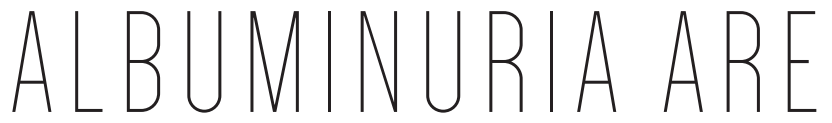

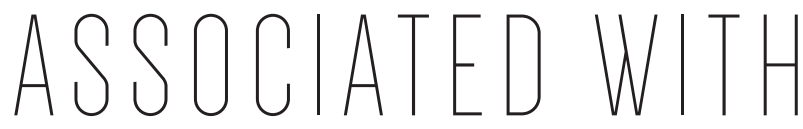

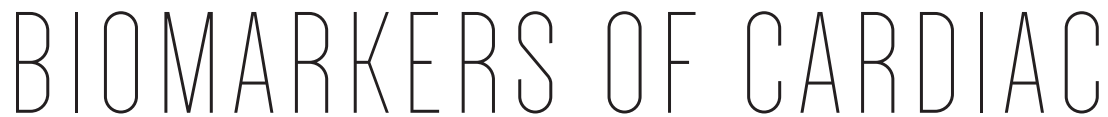

INJURY IN A POPULATION-

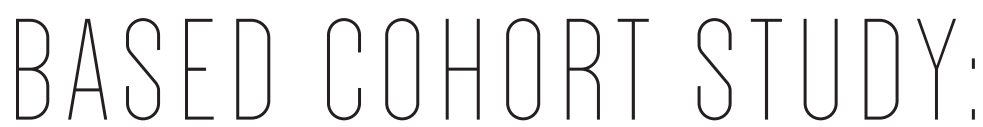

THE MAASTRICHT STUDY

RemyJH Martens, Dorien M Kimenai, Jeroen P Kooman, Coen DA Stehouwer, Frans ES Tan, Otto Bekers, Pieter C Dagnelie, Carla JH van der Kallen, Abraham A Kroon, Karel ML Leunissen, Frank M van der Sande, Nicolaas C Schaper, Simone JS Sep, Miranda T Schram, Jeroen D van Suijlen, Marja P van Dieijen-Visser, Steven JR Meex, Ronald MA Henry

Clinical Chemistry 2017; 63 (4): 887-897 


\section{ABSTRACT}

\section{$>$ BACKGROUND}

Chronic kidney disease (CKD) is associated with an increased cardiovascular disease mortality risk. It is, however, less clear at what point in the course from normal kidney function to CKD the association with cardiovascular disease appears. Studying the associations of estimated glomerular filtration rate (eGFR) and albuminuria with biomarkers of (subclinical) cardiac injury in a population without substantial CKD may clarify this issue.

\section{$>$ METHODS}

We examined the cross-sectional associations of eGFR and urinary albumin excretion (UAE) with high-sensitivity cardiac troponin (hs-cTn) T, hs-cTnI, and N-terminal probrain natriuretic-peptide (NT-proBNP) in 3103 individuals from a populationbased diabetes-enriched cohort study.

\section{$>$ RESULTS}

After adjustment for potential confounders, eGFR and UAE were associated with these biomarkers of cardiac injury, even at levels that do not fulfill the CKD criteria. For example, eGFR $60-<90 \mathrm{~mL} / \mathrm{min} / 1.73 \mathrm{~m}^{2}\left[\mathrm{vs} \geq 90 \mathrm{~mL} / \mathrm{min} / 1.73 \mathrm{~m}^{2}\right]$ was associated with a [ratio $(95 \% \mathrm{CI})] 1.21(1.17-1.26), 1.14(1.07-1.20)$, and $1.19(1.12-1.27)$ times higher hs-cTnT, hs-cTnI, and NT-proBNP, respectively. The association of eGFR with hs-cTnT was statistically significantly stronger than that with hs-cTnI. In addition, UAE $15-<30 \mathrm{mg} / 24 \mathrm{~h}$ (vs $<15 \mathrm{mg} / 24 \mathrm{~h}$ ) was associated with a 1.04 (0.98 - 1.10), 1.08 $(1.00-1.18)$, and $1.07(0.96-1.18)$ times higher hs-cTnT, hs-cTnI, and NT-proBNP, respectively.

\section{$>$ CONCLUSIONS}

eGFR and albuminuria were already associated with biomarkers of (subclinical) cardiac injury at levels that do not fulfill the CKD criteria. Although reduced renal elimination may partly underlie the associations of eGFR, these findings support the concept that eGFR and albuminuria are, over their entire range, associated with cardiac injury. 


\section{INTRODUCTION}

Chronic kidney disease (CKD), which is defined as an estimated glomerular filtration rate $(\mathrm{eGFR})<60 \mathrm{~mL} / \mathrm{min} / 1.73 \mathrm{~m}^{2}$ and/or a urinary albumin excretion (UAE) $\geq 30 \mathrm{mg} / 24 \mathrm{~h}$, is associated with an increased cardiovascular disease (CVD) mortality risk. ${ }^{1}$ It is, however, not entirely clear at what point in the course from normal kidney function to CKD the association with CVD risk appears. ${ }^{1,2}$ For example, GFR estimates that include cystatin $C$ have been associated with CVD mortality at levels far above the $60 \mathrm{~mL} / \mathrm{min} / 1.7 \mathrm{~m}^{2}$ threshold, whereas this is less clear for eGFR based on creatinine only. ${ }^{2}$ In addition, it is important to gain insight into the mechanisms that underlie the CVD mortality risk associated with eGFR and albuminuria. Studying the associations of eGFR levels $\geq 60 \mathrm{~mL} / \mathrm{min} / 1.73 \mathrm{~m}^{2}$ and UAE levels $<30 \mathrm{mg} / 24 \mathrm{~h}$ with biomarkers of (subclinical) cardiac injury, such as the cardiac troponins (cTn) $\mathrm{T}$ and I, and N-terminal probrain natriuretic-peptide (NT-proBNP) ${ }^{3,4}$, may help in clarifying these issues. The interpretation of these biomarkers in the face of kidney disease is, nevertheless, controversial. ${ }^{5}$

In this regard, it is important to distinguish eGFR from albuminuria as both entities may be associated with biomarkers of cardiac injury via different mechanisms. For instance, reduced eGFR may cause cardiac injury via chronic low-grade inflammation and endothelial dysfunction ${ }^{6}$, but may also reduce the renal elimination of cTn and NT-proBNP. ${ }^{5,7}$ In contrast, albuminuria has been hypothesized to be a biomarker of generalized endothelial dysfunction ${ }^{8}$ and capillary rarefaction ${ }^{9}$, which in turn may cause (subclinical) cardiac ischemia ${ }^{10}$, resulting in higher cTn and NT-proBNP concentrations. ${ }^{3,4}$ Indeed, the limited available data ${ }^{5,11-13}$ suggest that eGFR and albuminuria are mutually independently associated with $\mathrm{cTn}^{5,11,13}$ and NT-proBNP. ${ }^{11}$ However, only 2 of these studies have examined a population without substantial CKD. ${ }^{11,13}$

In addition, only 2 studies have examined whether cTnT and cTnI behave similarly with regard to their associations with eGFR at levels $\geq 60 \mathrm{~mL} / \mathrm{min} / 1.73 \mathrm{~m}^{2,14}$ and with albuminuria. ${ }^{5}$ This may be important as both cTn are used interchangeably in clinical practice, whereas CTnT has been suggested to be more strongly dependent on renal elimination than $\mathrm{CTnI} .{ }^{15}$ The latter is supported by stronger associations of eGFR $^{5,15-18}$ and measured GFR ${ }^{19}$ with cTnT than with cTnI at levels $<60 \mathrm{~mL} / \mathrm{min} / 1.73 \mathrm{~m}^{2}$, even when both cTn were measured with high-sensitivity (hs) assays. , $, 15,17,19^{19}$

In view of the above, we examined whether eGFR and albuminuria were, independently of each other, associated with hs-cTnT, hs-cTnI, and NT-proBNP in a population-based cohort, and whether these associations appeared at levels of eGFR and albuminuria, which do not fulfill the CKD criteria. In addition, we compared the strength of any associations of eGFR and albuminuria with hs-cTnT and hs-cTnI. 


\section{MATERIALS AND METHODS}

\section{> THE MAASTRICHT STUDY POPULATION AND DESIGN}

We used data from The Maastricht Study, an observational prospective populationbased cohort study. The rationale and methodology have been described previously. ${ }^{20}$ In brief, the study focuses on the etiology, pathophysiology, complications, and comorbidities of type 2 diabetes mellitus (T2DM) and is characterized by an extensive phenotyping approach. Eligible for participation were all individuals aged between 40 and 75 years and living in the southern part of the Netherlands. Participants were recruited through mass media campaigns and from the municipal registries and the regional Diabetes Patient Registry via mailings. Recruitment was stratified according to known $\mathrm{T}_{2} \mathrm{DM}$ status, with an oversampling of individuals with $\mathrm{T}_{2} \mathrm{DM}$, for reasons of efficiency.

The present report includes cross-sectional data from the first 3451 participants, who completed the baseline survey between November, 2010 and September, 2013. The examinations of each participant were performed within a time window of 3 months. The study has been approved by the institutional medical ethical committee (NL31329.068.10) and the Minister of Health, Welfare and Sports of the Netherlands (Permit 131088-105234-PG). All participants gave written informed consent.

For this study, participants with type 1 diabetes or other specific types of diabetes (n = 41) were excluded. After further successively excluding participants whose eGFR based on creatinine and cystatin (eGFR crcys $_{\text {s }}$ ) was missing $(\mathrm{n}=33$ ), whose 24 -h urine collections were collected erroneously ( $<2$ oh or $>28 \mathrm{~h}$ ) or were not handed in at all (n $=42$ ), and who had missing data on other variables in our primary regression models $(\mathrm{n}=232), 3103$ participants were included.

\section{$>$ KIDNEY FUNCTION}

GFR was estimated with the CKD-EPI equation based on the combination of serum creatinine and serum cystatin C (eGFR crcys ) for the primary analyses (Supplemental Methods). ${ }^{21}$ For additional analyses, eGFR was calculated with the CKD-EPI equations based on serum creatinine $\left(e G F R_{c r}\right)^{21}$ and serum cystatin $\mathrm{C}(\mathrm{eGFR}$ cys $){ }^{21}$ To assess UAE, participants were requested to collect two 24-h urine collections (Supplemental Methods). UAE was preferably based on the average of 2 (available in $91.9 \%$ of the participants) 24-h urine collections.

\section{$>$ CARDIAC BIOMARKERS}

Fasting blood samples were collected from all participants and processed according to the manufacturers' instructions. Serum samples were stored at $-80^{\circ} \mathrm{C}$ for $1-4$ years. hs-cTnT was measured on a Roche Cobas 6000 analyzer (Roche) with the Elecsys Troponin Ths assay (Roche), which has a limit of blank (LoB) of $3 \mathrm{ng} / \mathrm{L}$, a limit of detection (LoD) of $5 \mathrm{ng} / \mathrm{L}$, and achieves a 10\% coefficient of variation (10\% CV) at 13 
ng/L. hs-cTnI was measured on an Architect i20oo SR analyzer (Abbott Diagnostics) with the Architect STAT High Sensitive Troponin-I assay (Abbott), which has an LoB range of $0.7-1.3 \mathrm{ng} / \mathrm{L}$, an LoD range of $1.1-1.9 \mathrm{ng} / \mathrm{L}$, and achieves a 10\% CV at 4.7 ng/L. NT-proBNP was assessed on a Roche Cobas 6000 analyzer (Roche) with the Elecsys proBNP II assay (Roche), which has a LoD of $5.0 \mathrm{ng} / \mathrm{L}$ (LoB not reported), and achieves a $20 \% \mathrm{CV}$ at $50.0 \mathrm{ng} / \mathrm{L}$.

\section{$>$ COVARIATES}

We collected data on glucose metabolism status, hemoglobin $\mathrm{A}_{1 \mathrm{c}}\left(\mathrm{Hb} \mathrm{A}_{1 \mathrm{c}}\right)$, total cholesterol, HDL, LDL, triglycerides, waist circumference, office blood pressure, 24-h average ambulatory blood pressure, medication use, smoking behavior, alcohol consumption, educational level, questionnaire based prevalent CVD, resting 12lead electrocardiogram (ECG), and self-reported physical activity as described previously. ${ }^{20,22}$ See the Supplemental Methods for further details and definitions.

\section{$>$ STATISTICAL ANALYSES}

All analyses were performed with SPSS Statistics version 22.0 (IBM). Characteristics of the entire study population, and as categorized according to GFR $_{\text {crcys }}$ and albuminuria, were summarized as means with SD, medians with interquartile ranges (IQRs), and numbers with percentages, as appropriate. The cardiac biomarkers are described with both actual mean (with SD) and median (with IQR) levels. However, given their positively skewed distributions, the median and IQR most accurately capture their central tendency and variation.

For the comparison of participants across eGFR crcys and albuminuria categories, continuous variables were analyzed with one-way ANOVA if normally distributed and with the Kruskal-Wallis test if not. Categorical variables were compared with the $\mathrm{x}^{2}$ test.

Associations of eGFR $\mathrm{crcys}_{\text {s }}$ and albuminuria with hs-cTnT, hs-cTnI, and NT-proBNP were evaluated with multivariable linear regression analyses. eGFR $\mathrm{Crcys}_{\text {s }}$ and albuminuria were analyzed as categorical $\left(\geq 90,60-<90\right.$, and $<60 \mathrm{~mL} / \mathrm{min} / 1.73 \mathrm{~m}^{2}$; $<$ $15,15-<30$, and $\geq 30 \mathrm{mg} / 24 \mathrm{~h}$ ) and as continuous (per $-10 \mathrm{~mL} / \mathrm{min} / 1.73 \mathrm{~m}^{2}$; per doubling of UAE) variables. To obtain similar proportions of individuals with a value above the LoB for hs-cTnT and hs-cTnI ( $87.1 \%$, and $87.9 \%$, respectively), the LoB of hscTnI was considered to be $0.9 \mathrm{ng} / \mathrm{L}$. For both hs-cTnT and hs-cTnI, concentrations below the LoB were subsequently set at LoB/2. NT-proBNP concentrations below the LoD were set at LoD/2. Thereafter, concentrations of hs-cTnT, hs-cTnI, and NT-proBNP were natural logarithmically transformed. The regression coefficients were exponentiated to obtain the ratio of (geometric mean) concentrations of the cardiac biomarkers per 1 unit increase in the independent variable. We adjusted for potential confounders as follows: model 1, unadjusted model; model 2, age, sex, glucose metabolism status; model 3, model $2+$ waist circumference, total-toHDL cholesterol ratio, triglycerides, use of lipid-modifying medication, smoking 


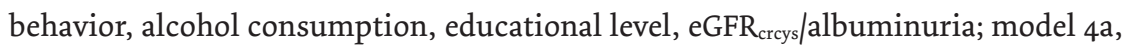
model 3 + office systolic pressure, use of antihypertensive medication, ischemic ECG abnormalities; model 4 b, similar to model 4 a but adjusted for 24 -h average ambulatory systolic pressure instead of office systolic pressure. Systolic pressure, use of antihypertensive medication, and ischemic ECG abnormalities were entered in a separate model as these may be both confounders and intermediates in the associations examined. The distributions of hs-cTnT and hs-cTnI should be taken into account when comparing their associations with eGFR crcys $_{\text {and }}$ albuminuria. Therefore, we subsequently calculated partial correlations of eGFR $_{\text {crcys }}$ and albuminuria with hs-cTnT and hs-cTnI in model $4 \mathrm{a}$ and compared these with a test for comparing correlations measured on the same individuals. ${ }^{23}$

Several additional analyses were performed. First, analyses were repeated: in participants with $\mathrm{UAE} \leq 300 \mathrm{mg} / 24 \mathrm{~h}$ and in participants with 2 valid urine collections; with additional adjustment for total and moderate to vigorous physical activity; and with alternative operationalization's of prior CVD, blood pressure, and the use of antihypertensive medication. Second, eGFR crcys $_{\text {was }}$ replaced with either eGFR $_{\text {cr }}$ or eGFR $_{\text {cys }}$. Third, we added interaction terms to model 4a to explore interaction with glucose metabolism status because glucose metabolism status has been shown to modify associations of other CVD risk factors with CVD events ${ }^{24}\left(P_{\text {interaction }}<0.10\right.$ was considered statistically significant). Fourth, to explore whether any association of eGFR $_{\text {crys }}$ and albuminuria with (subclinical) cardiac injury could also be detected with an ECG, we examined their associations with ischemic ECG abnormalities. 


\section{RESULTS}

\section{$>$ CHARACTERISTICS OF THE STUDY POPULATION}

Table 6.1 and Supplemental Table 6.1 show the characteristics of the study population stratified according to eGFR $\mathrm{Grcys}_{\text {s }}$ and albuminuria categories, respectively. Due to oversampling, $27.7 \%$ had T2DM.

Average eGFR crcys $_{\text {was }} 88.2(14.8) \mathrm{mL} / \mathrm{min} / 1.73 \mathrm{~m}^{2}$. Most participants had an $\mathrm{eGFR}_{\text {crcys }} \geq 90 \mathrm{~mL} / \mathrm{min} / 1.73 \mathrm{~m}^{2}(48.2 \%)$ or $60-<90 \mathrm{~mL} / \mathrm{min} / 1.73 \mathrm{~m}^{2}(47.7 \%)$, whereas $4.1 \%$ had an eGFR crcys $<60 \mathrm{~mL} / \mathrm{min} / 1.73 \mathrm{~m}^{2}$. Median UAE was 6.7 [IQR 4.1-11.9] mg/24h. In $10.4 \%$ UAE was 15 to $<30 \mathrm{mg} / 24 \mathrm{~h}$, whereas it was $\geq 30 \mathrm{mg} / 24 \mathrm{~h}$ in $8.6 \%$.

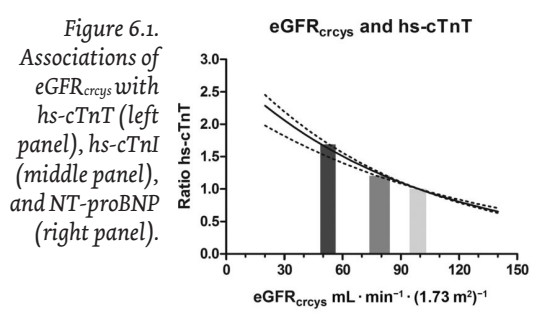

11. $290 \mathrm{~mL} \cdot \min ^{-1} \cdot\left(1.73 \mathrm{~m}^{2}\right)^{-1}$

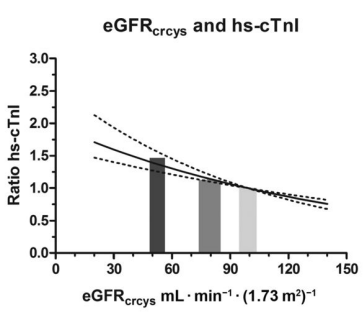

$60-<90 \mathrm{~mL} \cdot \min ^{-1} \cdot\left(1.73 \mathrm{~m}^{2}\right)^{-1}$

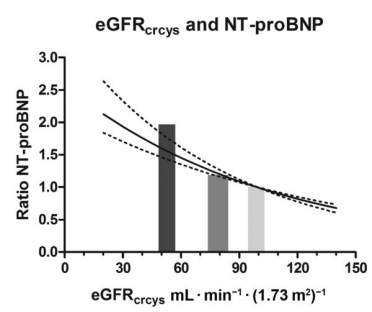

$<60 \mathrm{~mL} \cdot \mathrm{min}^{-1} \cdot\left(1.73 \mathrm{~m}^{2}\right)^{-1}$

eGFR crrys is expressed as a continuous variable (continuous lines with dashed lines for $95 \%$ CIs) and as a categorical

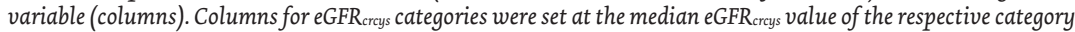
(column width represents IQR). Ratios represent the ratio of geometric mean concentrations of cardiac biomarkers as compared with an eGFRcrcys of $99.2 \mathrm{~mL} / \mathrm{min} / 1.73 \mathrm{~m}^{2}$ [the median eGFR crys in participants with eGFR crcys $\geq 90 \mathrm{~mL}$ / $\mathrm{min} / 1.73 \mathrm{~m}^{2}$ for eGFR crys expressed as a continuous variable or as compared with eGFR creys $\geq 90 \mathrm{~mL} / \mathrm{min} / 1.73 \mathrm{~m}^{2}$ for eGFR crys expressed as a categorical variable (model $4 a$ ).

In general, participants with lower eGFR crcys $_{\text {and }}$ participants with higher UAE had a worse CVD risk profile. Further, lower eGFR crcys was accompanied by higher UAE and vice versa. Supplemental Figure 6.1 shows the distribution of hs-cTnT and hs-cTnI. hs-cTnT was above the LoD in 55.6\%; hs-cTnI was above the LoD in $49.3 \%$ $(1.9 \mathrm{ng} / \mathrm{L})$ to $80.2 \%(1.1 \mathrm{ng} / \mathrm{L})$.

\section{$>$ EGFR AND CARDIAC BIOMARKERS}

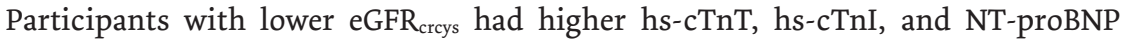
(Table 6.1). After adjustment for potential confounders, and as compared with eGFR crcys $_{2} \geq 90 \mathrm{~mL} / \mathrm{min} / 1.73 \mathrm{~m}^{2}$, both eGFR crcys $60-<90 \mathrm{~mL} / \mathrm{min} / 1.73 \mathrm{~m}^{2}$ and eGFR crcys $_{\text {s }}$ $<60 \mathrm{~mL} / \mathrm{min} / 1.73 \mathrm{~m}^{2}$ remained associated with higher hs-cTnT, hs-cTnI, and NTproBNP (Table 6.2, model 4a). Results were similar when we adjusted for 24 -h average ambulatory systolic pressure (Table 6.2, model 4 b). 
When eGFR crys $_{\text {was }}$ expressed as a continuous variable, each $10 \mathrm{~mL} / \mathrm{min} / 1.73 \mathrm{~m}^{2}$ lower eGFR $_{\text {crys }}$ was associated with a [ratio $\left.(95 \% \mathrm{CI})\right] 1.11(1.09-1.12)$ times higher hs-cTnT, a $1.07(1.05-1.10)$ times higher hs-cTnI, and a $1.10(1.08-1.13)$ times higher NT-proBNP (model 4a). However, these models somewhat underestimated the associations at

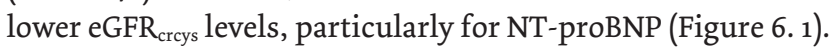

\section{> EGFR AND CARDIAC BIOMARKERS - HS-CTNT VS. HS-CTNI}

The associations of categorical and continuous eGFR crcys with hs-cTnT were slightly stronger than those with hs-cTnI (Table 6.2, model 4a). This difference was statistically significant when we took their different distributions into account using a comparison of partial correlations in model $4 \mathrm{a}\left(\mathrm{P}<0.001\right.$ for eGFR crcys $_{6} 60-<$ $90 \mathrm{~mL} / \mathrm{min} / 1.73 \mathrm{~m}^{2}, \mathrm{eGFR}_{\text {crcys }}<60 \mathrm{~mL} / \mathrm{min} / 1.73 \mathrm{~m}^{2}$, and continuous eGFR crcys $_{\text {) }}$.

\section{$>$ ALBUMINURIA AND CARDIAC BIOMARKERS}

Participants with higher UAE had higher hs-cTnT, hs-cTnI, and NT-proBNP (Supplemental Table 6.1). After adjustment for potential confounders, and as compared with UAE $<15 \mathrm{mg} / 24 \mathrm{~h}$, both UAE $15-<30 \mathrm{mg} / 24 \mathrm{~h}$ and UAE $\geq 30 \mathrm{mg} / 24 \mathrm{~h}$ remained associated with higher hs-cTnI, whereas only UAE $\geq 30 \mathrm{mg} / 24 \mathrm{~h}$ was associated with higher hs-cTnT and NT-proBNP (Table 6.3, model 4a). Results were similar when we adjusted for 24-h average ambulatory systolic pressure (Table 6.3, model 4 b).

When albuminuria was expressed as a continuous variable, each doubling of UAE was associated with a [ratio $(95 \% \mathrm{CI})] 1.03(1.02-1.04)$ times higher hs-cTnT, a 1.05 $(1.03-1.07)$ times higher hs-cTnI, and a $1.07(1.04-1.09)$ times higher NT-proBNP (model 4a). However, these models somewhat underestimated the associations at higher UAE, particularly for hs-cTnI and NT-proBNP (Figure 6.2). 


\begin{tabular}{|c|c|c|c|}
\hline $\begin{array}{r}\text { Table 6.1. } \\
\text { Clinical }\end{array}$ & & Study population, $n=3103$ & $\begin{array}{l}\text { eGFRcrcys categories } \\
\triangleq 90 \mathrm{ml} / \mathrm{min} / \mathrm{I} .73 \mathrm{~m}^{2}, \mathrm{n}=1497\end{array}$ \\
\hline characteristics & Demographics & & \\
\hline of the study & Age, years & $59.8 \pm 8.2$ & $56.5 \pm 8.1$ \\
\hline population & Men & $1608(51.8)$ & $763(51.0)$ \\
\hline stratified & Educational level & & \\
\hline according & Low & $517(16.7)$ & 207 (I3.8) \\
\hline to eGFR creys & Intermediate & $1327(42.8)$ & $664(44.4)$ \\
\hline categories. $^{a}$ & High & $1259(40.6)$ & $626(41.8)$ \\
\hline & Ischemic ECG abnormalities & $333(10.7)$ & $150(10.0)$ \\
\hline & Questionnaire based prevalent cardiovascular disease ${ }^{c}$ & $504(16.5)$ & $186(12.6)$ \\
\hline & Lifestyle variables & & \\
\hline & Smoking behavior & & \\
\hline & Never smoker & $1088(35.1)$ & $550(36.7)$ \\
\hline & Former smoker & $1596(51.4)$ & $751(50.2)$ \\
\hline & Current smoker & $419(13.5)$ & $196(\mid 3.1)$ \\
\hline & Alcohol consumption & & \\
\hline & None & $572(18.4)$ & $269(18.0)$ \\
\hline & Low (women $\leq 7$ glasses/week; men $\leq 14$ glasses/week) & $1720(55.4)$ & $827(55.2)$ \\
\hline & High (women > 7 glasses/week; men > 14 glasses/week) & $811(26.1)$ & $401(26.8)$ \\
\hline & Metabolic variables & & \\
\hline & Waist circumference, cm & & \\
\hline & Men & $101.4 \pm 11.9$ & $99.8 \pm 11.9$ \\
\hline & Women & $89.9 \pm 13.0$ & $88.4 \pm 12.7$ \\
\hline & Office systolic pressure, mmHg & $135.1 \pm 18.2$ & $133.3 \pm 17.4$ \\
\hline & Office diastolic pressure, mmHg & $76.2 \pm 9.9$ & $76.1 \pm 9.6$ \\
\hline & 24h average ambulatory systolic pressure, $\mathrm{mmHg}{ }^{d}$ & $1 \mid 9.1 \pm 11.8$ & $118.5 \pm 11.8$ \\
\hline & 24h average ambulatory diastolic pressure, $\mathrm{mmHg}{ }^{d}$ & $73.5 \pm 7.2$ & $73.9 \pm 7.1$ \\
\hline & Hypertension & $1742(56.1)$ & $735(49.1)$ \\
\hline & Glucose metabolis m status & & \\
\hline & Normal glucose metabolism & $1775(57.2)$ & $916(61.2)$ \\
\hline & Impaired fasting glucose & $129(4.2)$ & $62(4.1)$ \\
\hline & Impaired glucose tolerance & $341(11.0)$ & $156(10.4)$ \\
\hline & Type 2 diabetes & $858(27.7)$ & $363(24.2)$ \\
\hline & HDAÍC $(\%)^{e}$ & & \\
\hline & Without type 2 diabetes & $5.5 \pm 0.4$ & $5.5 \pm 0.4$ \\
\hline & With type 2 diabetes & $6.9 \pm 1.0$ & $6.9 \pm 1.1$ \\
\hline & Total cholesterol, mg/dL & $202.7 \pm 44.8$ & $202.2 \pm 43.9$ \\
\hline & HDL cholesterol, mg/dL & & \\
\hline & Men & $51.4 \pm 14.5$ & $52.6 \pm 14.8$ \\
\hline & Women & $67.2 \pm 18.5$ & $68.1 \pm 18.6$ \\
\hline & LDL cholesterol, mg/dL & $119.5 \pm 40.0$ & $\| 9.1 \pm 39.2$ \\
\hline & Triglycerides, mg/dL & $107.2(77.9-152.3)$ & $100.1(72.6-\mid 43.5)$ \\
\hline & Total-to-HDL cholesterol ratio & $3.7 \pm 1.2$ & $3.6 \pm 1.2$ \\
\hline & Kidney function & & \\
\hline & eGFRcrcys, $\mathrm{ml} / \mathrm{min} / \mathrm{I} .73 \mathrm{~m}^{2}$ & $88.2 \pm 14.8$ & $99.2(94.3-104.5)$ \\
\hline & eGFRcr, $\mathrm{ml} / \mathrm{min} / \mathrm{l} .73 \mathrm{~m}^{2}$ & $84.5 \pm 13.9$ & $94.5(89.2-99.9)$ \\
\hline & eGFRcys, $\mathrm{ml} / \mathrm{min} / \mathrm{l} .73 \mathrm{~m}^{2}$ & $89.8 \pm 16.5$ & $102.4(96.6-107.9)$ \\
\hline & UAE, mg/24h & $6.7(4.1-11.9)$ & $6.4(3.9-10.7)$ \\
\hline & UAE categories & & \\
\hline & $<15 \mathrm{mg} / 24 \mathrm{~h}$ & $2514(81.0)$ & $|26|(84.2)$ \\
\hline & $15-<30 \mathrm{mg} / 24 \mathrm{~h}$ & $323(10.4)$ & $148(9.9)$ \\
\hline & $\geq 30 \mathrm{mg} / 24 \mathrm{~h}$ & $266(8.6)$ & $88(5.9)$ \\
\hline & Medication & & \\
\hline & Antihypertensive medication & $1223(39.4)$ & $469(31.3)$ \\
\hline & Renin-angiotensin system inhibitor & $927(29.9)$ & $350(23.4)$ \\
\hline & Lipid-modifying medication & $1104(35.6)$ & $454(30.3)$ \\
\hline & Cardiac biomarkers & & \\
\hline & hs-cTnT, ng/L & $\begin{array}{l}6.6 \pm 6.1 \\
5.4(3.9-7.9)\end{array}$ & $\begin{array}{l}5.1 \pm 3.1 \\
4.6(3.2-6.5)\end{array}$ \\
\hline & hs-cTnl, ng/L & $3.2 \pm 9.1$ & $2.5 \pm 5.9$ \\
\hline & & $1.9(1.3-3.1)$ & $1.7(1.1-2.6)$ \\
\hline & NT-proBNP, ng/L & $82.6 \pm 133.2$ & $60.5 \pm 63.8$ \\
\hline & & $6.0(3.4-10.7)$ & $5.0(2.8-8.7)$ \\
\hline
\end{tabular}




\begin{tabular}{|c|c|c|}
\hline $\begin{array}{l}\text { eGFRcrcys categories } \\
60-<90 \mathrm{ml} / \mathrm{min} / \mathrm{I} .73 \mathrm{~m}^{2}, \mathrm{n}=1480\end{array}$ & $\begin{array}{l}\text { eGFRcrcys categories } \\
<60 \mathrm{ml} / \mathrm{min} / \mathrm{l} .73 \mathrm{~m}^{2}, \mathrm{n}=126\end{array}$ & \\
\hline & & Pvalue $^{b}$ \\
\hline $62.6 \pm 7.1$ & $67.2 \pm 6.2$ & $<0.001$ \\
\hline 770 (52.0) & $75(59.5)$ & 0.18 \\
\hline $\begin{array}{l}271(18.3) \\
612(41.4) \\
597(40.3)\end{array}$ & $\begin{array}{l}39(31.0) \\
51(40.5) \\
36(28.6)\end{array}$ & $<0.001$ \\
\hline 157 (10.6) & $26(20.6)$ & $<0.01$ \\
\hline $270(18.6)$ & $48(38.7)$ & $<0.001$ \\
\hline $\begin{array}{l}507(34.3) \\
772(52.2) \\
201(13.6)\end{array}$ & $\begin{array}{l}31(24.6) \\
73(57.9) \\
22(17.5)\end{array}$ & 0.07 \\
\hline $\begin{array}{l}265(17.9) \\
824(55.7) \\
391(26.4)\end{array}$ & $\begin{array}{l}38(30.2) \\
69(54.8) \\
19(15.1)\end{array}$ & $<0.01$ \\
\hline
\end{tabular}

\begin{tabular}{|c|c|c|}
\hline $\begin{array}{l}102.3 \pm 11.4 \\
91.1 \pm 13.0\end{array}$ & $\begin{array}{l}109.3 \pm 12.5 \\
96.5 \pm 13.5\end{array}$ & $\begin{array}{l}<0.001 \\
<0.001\end{array}$ \\
\hline $136.3 \pm 18.7$ & $141.7 \pm 19.5$ & $<0.001$ \\
\hline $76.6 \pm 10.0$ & $74.5 \pm 10.9$ & 0.05 \\
\hline $119.5 \pm 11.8$ & $|2| . \mid \pm 11.9$ & 0.02 \\
\hline $73.4 \pm 7.3$ & $70.7 \pm 6.7$ & $<0.001$ \\
\hline $890(60.1)$ & $117(92.9)$ & $<0.001$ \\
\hline $\begin{array}{l}827(55.9) \\
62(4.2) \\
174(11.8) \\
417(28.2)\end{array}$ & $\begin{array}{l}32(25.4) \\
5(4.0) \\
\| 1(8.7) \\
78(61.9)\end{array}$ & $<0.001$ \\
\hline $\begin{array}{l}5.6 \pm 0.4 \\
6.9 \pm 0.9\end{array}$ & $\begin{array}{l}5.6 \pm 0.4 \\
7.0 \pm 1.0\end{array}$ & $\begin{array}{l}<0.001 \\
0.47\end{array}$ \\
\hline $204.8 \pm 45.3$ & $183.9 \pm 44.8$ & $<0.001$ \\
\hline $\begin{array}{l}50.7 \pm 14.3 \\
66.8 \pm 18.4\end{array}$ & $\begin{array}{l}45.1 \pm 11.1 \\
60.2 \pm 17.1\end{array}$ & $\begin{array}{l}<0.001 \\
<0.01\end{array}$ \\
\hline $121.5 \pm 40.2$ & $102.2 \pm 40.8$ & $<0.001$ \\
\hline $110.7(84.1-155.0)$ & $138.2(98.3-203.3)$ & $<0.001$ \\
\hline $3.8 \pm 1.2$ & $3.8 \pm 1.1$ & $<0.01$ \\
\hline $80.1(73.6-85.6)$ & $53.0(48.6-57.5)$ & $<0.001$ \\
\hline $77.3(70.8-83.9)$ & $53.8(46.7-58.4)$ & $<0.001$ \\
\hline $80.8(73.1-87.9)$ & $52.6[(46.4-57.3)$ & $<0.001$ \\
\hline $6.7(4.1-12.3)$ & $14.4(7.0-68.6)$ & $<0.001$ \\
\hline $\begin{array}{l}1187(80.2) \\
156(10.5) \\
137(9.3)\end{array}$ & $\begin{array}{l}66(52.4) \\
19(15.1) \\
41(32.5)\end{array}$ & $<0.001$ \\
\hline 641 (43.3) & $113(89.7)$ & $<0.001$ \\
\hline $480(32.4)$ & $97(77.0)$ & $<0.001$ \\
\hline $561(37.9)$ & $89(70.6)$ & $<0.001$ \\
\hline $7.5 \pm 7.3$ & $13.8 \pm 9.8$ & $<0.001$ \\
\hline $6.0(4.4-8.8)$ & $11.3(7.3-17.2)$ & $<0.001$ \\
\hline $3.6 \pm 11.6$ & $5.4 \pm 7.1$ & $<0.001$ \\
\hline $2.1(1.4-3.4)$ & $3.7(2.5-5.5)$ & $<0.001$ \\
\hline $92.8 \pm 140.2$ & $225.6 \pm 362.5$ & $<0.001$ \\
\hline $6.8(4.0-11.7)$ & $12.0(6.9-28.8)$ & $<0.001$ \\
\hline
\end{tabular}

aData are presented as $n(\%)$, mean $\pm S D$, or median (interquartile range). Conversion factors for units: $\mathrm{Hb}$ Aic in \% to $\mathrm{mmol} / \mathrm{mol}, 10.93 \times$ $\mathrm{Hb}$ A1c (\%) +23.5; cholesterol in $\mathrm{mg} /$ $\mathrm{dL}$ to mmol, $\times 0.02586$; triglycerides in $\mathrm{mg} / \mathrm{dL}$ to $\mathrm{mmol} / \mathrm{L}, \times 0.01129$. ${ }^{b} P$ values for the comparison of participants across the eGFR crcys categories were calculated with the one-way ANOVA test for normally distributed variables, the KruskalWallis test for non-normally distributed variables and the 2 test for categorical variables. 'Data available for 3059 participants. ${ }^{d}$ Data available for 2749 participants. ${ }^{e}$ Data available for 3096 participants. 


\begin{tabular}{|c|c|c|c|c|c|c|c|c|}
\hline \multirow{2}{*}{$\begin{array}{r}\text { Table 6.2. } \\
\text { Associations of } \\
\text { eGFRcrcys with } \\
\text { biomarkers of } \\
\text { cardiac injury. }\end{array}$} & \multirow[b]{2}{*}{ Biomarker } & \multirow[b]{2}{*}{ Model } & \multicolumn{2}{|c|}{$\begin{array}{c}\operatorname{eGFR}_{\text {crrys }} \\
\geq 90 \mathrm{~mL} / \mathrm{min} / \mathrm{l} .73 \mathrm{~m}^{2}\end{array}$} & \multicolumn{2}{|c|}{$\begin{array}{c}\text { eGFR } \\
60 \text { torys } \\
<90 \mathrm{~mL} / \mathrm{min} / \mathrm{l} .73 \mathrm{~m}^{2}\end{array}$} & \multicolumn{2}{|c|}{$\begin{array}{c}\operatorname{eGFR}_{\text {creys }} \\
<60 \mathrm{~mL} / \mathrm{min} / \mathrm{l} .73 \mathrm{~m}^{2}\end{array}$} \\
\hline & & & Ratio (95\% Cl) & Pvalue & Ratio (95\% Cl) & Pvalue & Ratio (95\% Cl) & Pvalue \\
\hline & hs-cTnT & । & Reference & $N / A^{b}$ & $1.44(1.38-1.51)$ & $<0.001$ & $2.68(2.40-3.00)$ & $<0.001$ \\
\hline & & 2 & Reference & $N / A$ & $1.23(1.18-1.27)$ & $<0.001$ & $1.81(1.65-1.99)$ & $<0.001$ \\
\hline & & 3 & Reference & $N / A$ & I.21 (1.17- - ..26) & $<0.001$ & $1.73(1.57-1.90)$ & $<0.001$ \\
\hline & & $4 a$ & Reference & $N / A$ & I.21 (1.17- I.26) & $<0.001$ & $1.69(1.53-1.85)$ & $<0.001$ \\
\hline & & $4 b$ & Reference & $\mathrm{N} / \mathrm{A}$ & $1.21(1.16-1.26)$ & $<0.001$ & $1.70(1.54-1.88)$ & $<0.001$ \\
\hline & hs-cTnl & । & Reference & $N / A$ & $1.35(1.27-1.43)$ & $<0.001$ & $2.24(1.93-2.60)$ & $<0.001$ \\
\hline & & 2 & Reference & $\mathrm{N} / \mathrm{A}$ & $1.15(1.09-1.22)$ & $<0.001$ & $1.63(1.42-1.88)$ & $<0.001$ \\
\hline & & 3 & Reference & $\mathrm{N} / \mathrm{A}$ & I.13 (1.07- - 1.19) & $<0.001$ & $1.49(1.29-1.72)$ & $<0.001$ \\
\hline & & $4 a$ & Reference & $N / A$ & $1.14(1.07-1.20)$ & $<0.001$ & $1.45(1.26-1.66)$ & $<0.001$ \\
\hline & & $4 b$ & Reference & $N / A$ & $1.15(1.08-1.22)$ & $<0.001$ & $1.45(1.25-1.68)$ & $<0.001$ \\
\hline & NT-proBNP & I & Reference & $N / A$ & $1.40(1.32-1.50)$ & $<0.001$ & $2.82(2.39-3.33)$ & $<0.001$ \\
\hline & & 2 & Reference & $N / A$ & $1.17(1.09-1.25)$ & $<0.001$ & $2.15(1.82-2.54)$ & $<0.001$ \\
\hline & & 3 & Reference & $N / A$ & $1.19(1.11-1.27)$ & $<0.001$ & $2.05(1.74-2.43)$ & $<0.001$ \\
\hline & & $4 a$ & Reference & $N / A$ & I.19 (1.12 - I.27) & $<0.001$ & $1.94(1.64-2.29)$ & $<0.001$ \\
\hline & & $4 b$ & Reference & $\mathrm{N} / \mathrm{A}$ & $1.19(1.11-1.28)$ & $<0.001$ & $1.97(1.65-2.34)$ & $<0.001$ \\
\hline
\end{tabular}

${ }^{a}$ Betas represent the ratio of geometric mean concentrations of cardiac biomarkers in the respective eGFR crys $_{\text {category }}$ relative to participants with an eGFR $\mathrm{crrys}_{2} \geq 90 \mathrm{~mL} / \mathrm{min} / 1.73 \mathrm{~m}^{2}$. Model 1: unadjusted model; model 2: age, sex, glucose metabolism status; model 3: model $2+$ waist circumference, total-to-HDL cholesterol ratio, triglycerides, use of lipid-modifying medication, smoking behavior, alcohol consumption, educational level, UAE (categorical); model 4a: model 3 + office systolic pressure, use of antihypertensive medication, ischemic ECG abnormalities; model $4 \mathrm{~b}$ : similar to model 4 a but adjusted for 24-h average ambulatory systolic pressure instead of office systolic pressure (missing in 354 participants). ${ }^{b} \mathrm{NA}$, not applicable. 


\begin{tabular}{|c|c|c|c|c|c|c|c|}
\hline \multirow[b]{2}{*}{ Biomarker } & \multirow[b]{2}{*}{ Model } & \multicolumn{2}{|c|}{ UAE $<15 \mathrm{mg} / 24 \mathrm{~h}$} & \multicolumn{2}{|c|}{ UAE $15-<30 \mathrm{mg} / 24 \mathrm{~h}$} & \multicolumn{2}{|c|}{$U A E \equiv 30 \mathrm{mg} / 24 \mathrm{~h}$} \\
\hline & & Ratio (95\% Cl) & Pvalue & Ratio (95\% Cl) & Pvalue & Ratio (95\% CI) & Pvalue \\
\hline \multirow[t]{5}{*}{ hs-cTnT } & । & Reference & $N / A^{b}$ & $1.27(1.19-1.37)$ & $<0.001$ & $1.69(1.56-1.84)$ & $<0.001$ \\
\hline & 2 & Reference & $\mathrm{N} / \mathrm{A}$ & $1.05(0.99-1.12)$ & 0.10 & $1.22(\mid .14-1.31)$ & $<0.001$ \\
\hline & 3 & Reference & $N / A$ & $1.04(0.98-1.10)$ & 0.21 & $1.13(1.06-1.21)$ & $<0.001$ \\
\hline & $4 a$ & Reference & $N / A$ & $1.04(0.98-1.10)$ & 0.23 & $1.11(1.04-1.18)$ & $<0.01$ \\
\hline & $4 b$ & Reference & $\mathrm{N} / \mathrm{A}$ & $1.01(0.95-1.08)$ & 0.67 & $1.09(1.01-1.17)$ & 0.02 \\
\hline \multirow[t]{5}{*}{ hs-cTnl } & । & Reference & $N / A$ & $1.27(1.16-1.40)$ & $<0.001$ & $1.79(1.61-1.98)$ & $<0.001$ \\
\hline & 2 & Reference & $N / A$ & I.II (I.02 - I.2I) & 0.02 & I.41 (I.28 - I.55) & $<0.001$ \\
\hline & 3 & Reference & $N / A$ & $1.10(1.01-1.19)$ & 0.04 & $1.34(1.21-1.47)$ & $<0.001$ \\
\hline & $4 a$ & Reference & $\mathrm{N} / \mathrm{A}$ & $1.08(1.00-1.18)$ & 0.07 & $1.26(1.14-1.38)$ & $<0.001$ \\
\hline & $4 b$ & Reference & $\mathrm{N} / \mathrm{A}$ & $1.07(0.97-1.17)$ & 0.16 & I.21 (I.09 - I.34) & $<0.001$ \\
\hline \multirow[t]{5}{*}{ NT-proBNP } & । & Reference & $N / A$ & $1.08(0.97-1.21)$ & 0.15 & $1.51(1.34-1.70)$ & $<0.001$ \\
\hline & 2 & Reference & $N / A$ & $1.05(0.95-1.17)$ & 0.32 & $1.52(1.35-1.70)$ & $<0.001$ \\
\hline & 3 & Reference & $\mathrm{N} / \mathrm{A}$ & $1.06(0.96-1.17)$ & 0.28 & $1.41(1.26-1.58)$ & $<0.001$ \\
\hline & $4 a$ & Reference & $\mathrm{N} / \mathrm{A}$ & $1.07(0.96-1.18)$ & 0.21 & $1.34(1.19-1.50)$ & $<0.001$ \\
\hline & $4 b$ & Reference & $N / A$ & $1.04(0.94-1.16)$ & 0.43 & $1.29(1.15-1.46)$ & $<0.001$ \\
\hline
\end{tabular}

Table 6.3. Associations of albuminuria with biomarkers of cardiac injury. ${ }^{a}$

\footnotetext{
${ }^{a}$ Betas represent the ratio of geometric mean concentrations of cardiac biomarkers in the respective albuminuria category relative to participants with a $U A E<15 \mathrm{mg} / 24 \mathrm{~h}$. Model 1: unadjusted model; model 2: age, sex, glucose metabolism status; model 3: model $2+$ waist circumference, total-to-HDL cholesterol ratio, triglycerides, use of lipid-modifying medication, smoking behavior, alcohol consumption, educational level, eGFRcrcys (categorical); model 4a: model 3 + office systolic pressure, use of antihypertensive medication, ischemic ECG abnormalities; model $4 \mathrm{~b}$ : similar to model 4 a but adjusted for 24 -h average ambulatory systolic pressure instead of office systolic pressure (missing in 354 participants). ${ }^{b}$ NA, not applicable.
} 


\section{$>$ ALBUMINURIA AND CARDIAC BIOMARKERS - HS-CTNT VS. HS-CTNI}

The associations of categorical and continuous albuminuria with hs-cTnI seemed to be slightly stronger than those with hs-cTnT (Table 6.3, model 4a), but this difference was not statistically significant when we took the distributions of hs-cTnT and hs-cTnI into account using a comparison of partial correlations in model $4 \mathrm{a}(\mathrm{P}=0.57$ for $\mathrm{UAE} 15-<30 \mathrm{mg} / 24 \mathrm{~h} ; \mathrm{P}=0.13$ for $\mathrm{UAE} \leq 30 \mathrm{mg} / 24 \mathrm{~h} ; \mathrm{P}=0.72$ for continuous $\mathrm{UAE}$ ).
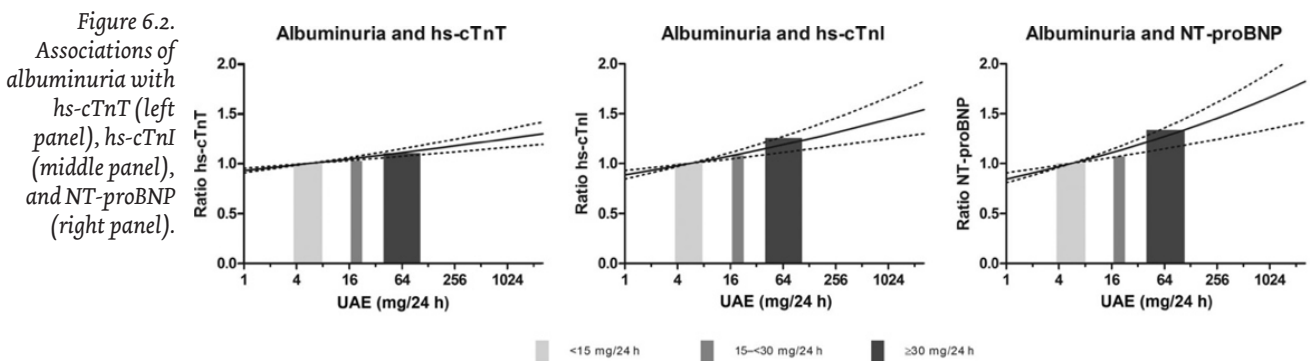

Albuminuria is expressed as a continuous variable (continuous lines with dashed lines for $95 \%$ CIs) and as a categorical variable (columns). Columns for urinary albumin excretion categories were set at the median urinary albumin excretion of the respective category (column width approximates the IQR). Ratios represent the ratio of geometric mean concentrations of cardiac biomarkers as compared with a urinary albumin excretion of $5.6 \mathrm{mg} / 24 \mathrm{~h}$ (the median urinary albumin excretion in participants with a urinary albumin excretion $<15 \mathrm{mg} / 24 \mathrm{~h}$ ) for albuminuria expressed as a continuous variable or as compared with a urinary albumin excretion $<15 \mathrm{mg} / 24 \mathrm{~h}$ for albuminuria expressed as a categorical variable (model $4 a)$.

\section{$>$ ADDITIONAL ANALYSES}

First, results were not materially altered: when we restricted the analyses to participants with $\mathrm{UAE} \leq 300 \mathrm{mg} / 24 \mathrm{~h}(\mathrm{n}=3081)$ or to participants with 2 urine collections $(n=2851)$; after additional adjustment for total $(n=2717)$ or moderate to vigorous physical activity $(\mathrm{n}=2716)$, or questionnaire based prevalent CVD $(n=3059)$; when we replaced office systolic pressure with either office diastolic pressure, office pulse pressure, office mean arterial pressure or their 24 -h average ambulatory equivalents, or the presence of hypertension; and when we replaced the use of antihypertensive medication with the use of a renin-angiotensin system inhibitor (data not shown).

Second, when eGFR crcys $_{\text {was }}$ replaced with eGFR cr, associations of eGFR with hs-cTnT and NT-proBNP became numerically weaker but remained statistically significant, whereas results were similar when eGFR crcys $_{\text {was }}$ waplaced with eGFR cys $_{\text {(Supplemental }}$ Table 6.2).

Third, analyses with interaction terms indicated statistically significant interaction $\left(\mathrm{P}_{\text {interaction }}<0.10\right)$ between $\mathrm{GFFR}_{\text {crcys }}$ and $\mathrm{T} 2 \mathrm{DM}$, to such an extent

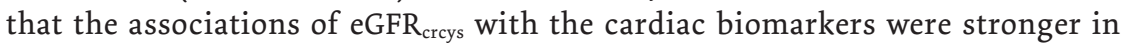


participants with T2DM than in participants with normal glucose metabolism (see stratified analyses in Supplemental Table 6.3). In addition, the associations of albuminuria with NT-proBNP were stronger in participants with T2DM than in participants with normal glucose metabolism (see stratified analyses in Supplemental Table 6.4).

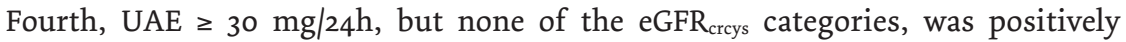
associated with ischemic ECG abnormalities after adjustment for potential confounders (see Supplemental Table 6.5).

\section{DISCUSSION}

This population-based study on the associations of eGFR and albuminuria with biomarkers of cardiac injury had two main findings. First, eGFR $\mathrm{ercys}_{\mathrm{s}}$ and albuminuria were mutually independently associated with hs-cTnT, hs-cTnI, and NT-proBNP after adjustment for demographics, lifestyle variables, and CVD risk factors. Second, eGFR $_{\text {crys }}$ was more strongly associated with hs-cTnT than with hs-cTnI. However, although statistically significant, this difference seemed modest in absolute terms. Of interest, for albuminuria the associations with hs-cTnT and hs-cTnI were much more similar.

Our results are in line with most ${ }^{5,11,13}$ but not all ${ }^{12}$ of the few previous studies that have conjointly evaluated eGFR and albuminuria and have shown mutually independent associations of both with hs-cTnT ${ }^{5,11,13}$, hs-cTnI ${ }^{5}$, and NT-proBNP. ${ }^{11}$ The present study expands the population-based data on this topic ${ }^{11,13}$ by its direct comparison of both cTn and the detailed characterization of its population, which allowed adjustment for an extensive series of potential confounders, including 24-h average ambulatory blood pressure. Altogether, the available data argue that eGFR and albuminuria are, over their entire range, associated with these biomarkers of cardiac injury.

cTn are released following myocardial injury due to, for example, cardiac ischemia, and possibly also cardiac strain. ${ }^{3}$ Similarly, NT-proBNP may be a marker of "pancardiac disease", being associated with left ventricular dysfunction, left ventricular hypertrophy, left atrial dilation, and cardiac ischemia. ${ }^{4}$ NT-proBNP $(8.5 \mathrm{kDa})$ is subsequently eliminated via the kidneys ${ }^{7}$, whereas the elimination routes of cTnT and cTnI are unknown. However, based on their molecular weight, free $\mathrm{cTnT}^{\mathrm{T}}(37 \mathrm{kDa})$, free $\mathrm{cTnI}(24 \mathrm{kDa})$, and their fragments may be (partly) filtered by the glomeruli and catabolized by the tubules. ${ }^{25,26}$ Indeed, after myocardial infarction, cTnT and cTnI have been observed as free cTnT and cTnI, larger cTnTI-C (77 kDa) and cTnI-C (40 kDa) complexes, and fragments..$^{27-31}$ In contrast, in renal failure, cTnT circulates primarily as fragments ${ }^{32}$, whereas the circulating form of cTnI in renal failure is unknown. 
In this study, we could not determine the relative contributions of lower renal elimination and cardiac injury to the association of eGFR with the cardiac biomarkers. The observation that eGFR seemed to be associated more strongly with the biomarkers than albuminuria, the absence of a positive association with ischemic ECG abnormalities and the minor attenuation of the regression coefficients after adjustment for these ECG findings may indicate a role for lower renal elimination. However, the cardiac biomarkers may identify cardiac injury that is subclinical and not visible on an ECG. Further, findings from other studies strongly argue that cardiac injury is involved. First, eGFR has been associated with CVD mortality at concentrations $\geq 60 \mathrm{~mL} / \mathrm{min} / 1.73 \mathrm{~m}^{2}$, especially if the GFR estimate included cystatin $\mathrm{C}^{2}$, and the evidence for an association of eGFR $<60 \mathrm{~mL}$ / $\min / 1.73 \mathrm{~m}^{2}$ with CVD is compelling., ${ }^{1,2}$ Second, hs-cTnT and NT-proBNP have been associated with incident overt CVD regardless of eGFR in the general population. ${ }^{11}$ Therefore, the results of this study suggest that (subclinical) cardiac injury may be a mechanism for the CVD mortality risk associated with lower eGFR in the general population.

In this study, eGFR was associated with the cardiac biomarkers regardless of the filtration marker and equation used. Nevertheless, associations of eGFR with hscTnT and NT-proBNP were numerically stronger with GFR estimates that included cystatin $C$. This observation agrees with data on the association of eGFR with CVD mortality. ${ }^{2}$ Non-GFR determinants of cystatin C, which may include obesity, diabetes, and inflammation, may have augmented the association with the cardiac biomarkers. ${ }^{2}$ Alternatively, cystatin C may be a more sensitive biomarker of GFR than creatinine in populations with more comorbidities. ${ }^{33}$

The association of albuminuria with the biomarkers of cardiac injury, which was independent of its association with ischemic ECG abnormalities, fits the strong association of albuminuria with CVD mortality. ${ }^{1}$ Generalized endothelial dysfunction and/or capillary rarefaction may explain the association of albuminuria with cardiac injury. According to this theory, endothelial dysfunction and capillary rarefaction in the microcirculation (i.e., micro vascular dysfunction) of the kidneys leads to albuminuria ${ }^{8,9}$, whereas in the heart both may cause a supply demand mismatch with (subclinical) ischemia. ${ }^{10}$ Indeed, lower coronary flow reserve ${ }^{34}$ and lower myocardial perfusion reserve ${ }^{35}$, which are measures of micro vascular dysfunction of the heart ${ }^{10}$, have been associated with higher $\mathrm{cTn}^{34}$ and NT-proBNP35 in individuals without overt coronary artery disease. Importantly, albuminuria has also been associated with lower coronary flow reserve. ${ }^{36}$

Explorative analyses suggested that the associations of eGFR with hs-cTnT, hscTnI, and NT-proBNP, and the association of albuminuria with NT-proBNP, were stronger in individuals with T2DM. Although speculative, these observations may have several explanations. First, the diabetic milieu may augment the effects of reduced eGFR on CVD. Second, experimental data suggest that albuminuria could 
be a better biomarker of micro vascular disease in individuals with diabetes than in non-diabetic individuals. ${ }^{37,38}$ This may be because hyperglycemia may disrupt the vascular endothelial growth factor (VEGF)-nitric oxide (NO) axis and, thereby, may aggravate vascular injury. ${ }^{39}$ Alternatively or additionally, in individuals with T2DM, glomerular hyper filtration ${ }^{40}$ may increase permeation of albumin through an injured glomerular capillary wall. Third, for NT-proBNP the interaction with T2DM may reflect the distributions of eGFR and UAE according to glucose metabolism status (i.e., lower eGFR and higher UAE in individuals with T2DM), combined with curvilinear associations of both with NT-proBNP (Figures 7.1 and 7.2). Nevertheless, we cannot exclude the play of chance.

Our finding that eGFR was more strongly associated with hs-cTnT than hs-cTnI expands the results of studies in selected populations ${ }^{5,14-19}$ to the range of eGFR observed in the general population. Differences in renal elimination between both $\mathrm{cTn}$, for example accumulation of cTnT fragments ${ }^{32}$, may explain their differential association with eGFR. ${ }^{15}$ However, other explanations should also be considered. For example, differences in stability and modification after release into the circulation that interfere with detection may exist in the face of reduced eGFR. ${ }^{41}$ Hence, future mechanistic studies should compare the extent of renal elimination of cTnT and cTnI. The similar associations of albuminuria with both cTn disagree with a smaller study that also showed stronger associations of albuminuria with hs-cTnT than hscTnI. ${ }^{5}$ However, that study did not formally compare both cTn taking their different distributions into account.

Our study had some limitations. First, owing to the cross-sectional design and the absence of data on hard clinical outcomes, such as incident CVD, we cannot make strong causal inferences and we cannot determine the direction of the associations. In fact, these may be bidirectional. ${ }^{42,43}$ In addition, data on hard clinical outcomes may clarify the clinical relevance of the differences between hs-cTnT and hs-cTnI. Second, although we measured cTnT and cTnI with hs assays, concentrations were still below the LoB and LoD in a substantial number of participants. Third, the lack of standardization of hs-cTnI assays may hamper generalizability to other hs-cTnI assays. Fourth, participants resided in a single region in the Netherlands, and the vast majority were white individuals from European descent (98.6\%); this may limit generalizability to other ethnic groups.

In conclusion, eGFR and albuminuria were, independently of each other, associated with biomarkers of (subclinical) cardiac injury, and these associations already appeared at levels that do not fulfill the CKD criteria. Although reduced renal elimination may partly underlie the associations of eGFR, this study supports the concept that eGFR and albuminuria are, over their entire range, associated with cardiac injury. In addition, eGFR was more strongly associated with hs-cTnT than with hs-cTnI. The mechanisms responsible for this difference need further study as this could be of clinical relevance. 
REFERENCES

1. Fox CS, Matsushita K, Woodward M, Bilo HJ, Chalmers J, Heerspink HJ, et al. Associations of kidney disease measures with mortality and end-stage renal disease in individuals with and without diabetes: a meta-analysis. Lancet 2012;380:1662-73.

2. Shlipak MG, Matsushita K, Arnlov J, Inker LA, Katz R, Polkinghorne KR, et al. Cystatin C versus creatinine in determining risk based on kidney function. N Engl J Med 2013;369:932- 43.

3. Giannitsis E, Katus HA. Cardiac troponin level elevations not related to acute coronary syndromes. Nat Rev Cardiol 2013;10:623-34.

4. Struthers A, Lang C. The potential to improve primary prevention in the future by using $\mathrm{BNP} / \mathrm{N}-\mathrm{BNP}$ as an indicator of silent 'pancardiac' target organ damage: BNP/N-BNP could become for the heart what microalbuminuria is for the kidney. Eur Heart J 2007;28:1678 - 82.

5. deFilippi C, Seliger SL, Kelley W, Duh SH, Hise M, Christenson RH, et al. Interpreting cardiac troponin results from high-sensitivity assays in chronic kidney disease without acute coronary syndrome. Clin Chem 2012;58:1342-51.

6. Schiffrin EL, Lipman ML, Mann JF. Chronic kidney disease: effects on the cardiovascular system. Circulation 2007;116:85-97.

7. van Kimmenade RR, Januzzi JL Jr, Bakker JA, Houben AJ, Rennenberg R, Kroon AA, et al. Renal clearance of B-type natriuretic peptide and amino terminal pro-Btype natriuretic peptide a mechanistic study in hypertensive subjects. J Am Coll Cardiol 2009;53:884-90.

8. Stehouwer CD, Smulders YM. Microalbuminuria and risk for cardiovascular disease: analysis of potential mechanisms. J Am Soc Nephrol 2006;17:2106 -11.

9. Martens RJ, Henry RM, Houben AJ, van der Kallen CJ, Kroon AA, Schalkwijk CG, et al. Capillary rarefaction associates with albuminuria: the MaastrichtStudy.J Am Soc Nephrol 2016;27:3748-57.

10. Camici PG, Crea F. Coronary microvascular dysfunction. N Engl J Med 2007;356:830 - 40.

11. Scheven L, de Jong PE, Hillege HL, Lambers Heerspink HJ, van Pelt LJ, Kootstra JE, et al. High-sensitive troponin $\mathrm{T}$ and $\mathrm{N}$-terminal pro-B type natriuretic peptide are associated with cardiovascular events despite the cross-sectional association with albuminuria and glomerular filtration rate. Eur Heart J 2012;33:2272- 81.

12. Dubin RF, Li Y, He J, Jaar BG, Kallem R, Lash JP, et al. Predictors of high sensitivity cardiac troponin $\mathrm{T}$ in chronic kidney disease patients: a cross-sectional study in the Chronic Renal Insufficiency Cohort (CRIC). BMC Nephrol 2013;14:229.

13. Xiao W, Ye P, Cao R, Yang X, Bai Y, Wu H. Urine albumin excretion is associated with cardiac troponin $\mathrm{T}$ detected with a highly sensitive assay in a community-based population. PloS one 2015;10:e0135747. 
14. Guclu T, Bolat S, Senes M, Yucel D. Relationship between high sensitivity troponins and estimated glomerular filtration rate. Clin Biochem 2016;49:467-71.

15. Cardinaels EP, Daamen MA, Bekers O, Ten Kate J, Niens M, van Suijlen JD, et al. Clinical interpretation of elevated concentrations of cardiac troponin $\mathrm{T}$, but not troponin $\mathrm{I}$, in nursing home residents. J Am Med Dir Assoc 2015;16:884 -91.

16. Lamb EJ, Kenny C, Abbas NA, John RI, Webb MC, Price CP, Vickery S. Cardiac troponin I concentration is commonly increased in nondialysis patients with CKD: experience with a sensitive assay. Am J Kidney Dis 2007;49:507-16.

17. Lippi G, Cervellin G. High-sensitivity troponin $\mathrm{T}$ is more susceptible than high-sensitivity troponin I to impaired renal function. Am J Cardiol 2013;112:1985.

18. Abbas NA, John RI, Webb MC, Kempson ME, Potter AN, Price CP, et al. Cardiac troponins and renal function in nondialysis patients with chronic kidney disease. Clin Chem 2005;51:2059- 66 .

19. Bjurman C, Petzold M, Venge P, Farbemo J, Fu ML, Hammarsten O. High-sensitive cardiac troponin, NTproBNP, hFABP and copeptin levels in relation to glomerular filtration rates and a medical record of cardiovascular disease. Clin Biochem 2015;48:302-7.

20. Schram MT, Sep SJ, van der Kallen CJ, Dagnelie PC, Koster A, Schaper N, et al. The Maastricht Study: an extensive phenotyping study on determinants of type 2 diabetes, its complications and its comorbidities. Eur J Epidemiol 2014;29:439 -51.

21. Inker LA, Schmid CH, Tighiouart H, Eckfeldt JH, Feldman HI, Greene T, et al. Estimating glomerular filtration rate from serum creatinine and cystatin C. N Engl J Med 2012;367:20 -9.

22. Spauwen PJ, van Boxtel MP, Verhey FR, Kohler S, Sep SJ, Koster A, et al. Both low and high 24-hour diastolic blood pressure are associated with worse cognitive performance in type 2 diabetes: The Maastricht Study. Diabetes Care 2015;38:1473- 80

23. Steiger JH. Tests for comparing elements of a correlation matrix. Psychol Bull 1980;87:245-51.

24. van Sloten TT, Henry RM, Dekker JM, Nijpels G, Unger T, Schram MT, Stehouwer CD. Endothelial dysfunction plays a key role in increasing cardiovascular risk in type 2 diabetes: The Hoorn study. Hypertension 2014;64:1299 -305.

25. Carone FA, Peterson DR, Oparil S, Pullman TN. Renal tubular transport and catabolism of proteins and peptides. Kidney Int 1979;16:271- 8 .

26. Maack T, Johnson V, Kau ST, Figueiredo J, Sigulem D. Renal filtration, transport, and metabolism of lowmolecular-weight proteins: a review. Kidney Int 1979;16:251-70.

27. Wu AH, Feng YJ, Moore R, Apple FS, McPherson PH, Buechler KF, Bodor G. Characterization of cardiac troponin subunit release into serum after acute myocardial infarction and comparison of assays for troponin T and I. American Association for Clinical Chemistry subcommittee on cTnI standardization. Clin Chem 1998;44:1198-208.

28. Cardinaels EP, Mingels AM, van Rooij T, Collinson PO, Prinzen FW, van Dieijen-Visser MP. Time-dependent degradation pattern of cardiac troponin $\mathrm{T}$ following myocardial infarction. Clin Chem 2013;59:1083-90.

29. Labugger R, Organ L, Collier C, Atar D, Van Eyk JE. Extensive troponin I and T modification detectedin serum from patients with acute myocardial infarction. Circulation 2000;102:1221-6.

30. Peronnet E, Becquart L, Poirier F, Cubizolles M, Choquet-Kastylevsky G, Jolivet-Reynaud C. SELDI-TOF MS analysis of the cardiac troponin I forms present in plasma from patients with myocardial infarction. Proteomics 2006;6:6288 -99. 
31. Katrukha AG, Bereznikova AV, Esakova TV, Pettersson K, Lovgren T, Severina ME, et al. Troponin I is released in bloodstream of patients with acute myocardial infarction not in free form but as complex. Clin Chem 1997;43:1379 - 85.

32. Mingels AM, Cardinaels EP, Broers NJ, Van Sleeuwen A, Streng AS, Van Dieijen-Visser MP, et al. Cardiac troponin $\mathrm{T}$ : smaller molecules in patients with end-stage renal disease than after onset of acute myocardial infarction. Clin Chem 2017;63:683-90.

33. Shlipak MG, Mattes MD, Peralta CA. Update on cystatin C: incorporation into clinical practice. Am J Kidney Dis 2013;62:595-603.

34. Taqueti VR, Everett BM, Murthy VL, Gaber M, Foster CR, Hainer J, et al. Interaction of impaired coronary flow reserve and cardiomyocyte injury on adverse cardiovascular outcomes in patients without overt coronary artery disease. Circulation 2015;131:528 -35.

35. Mitchell A, Misialek JR, Folsom AR, Duprez D, Alonso A, Jerosch-Herold M, et al. Usefulness of N-terminal probrain natriuretic peptide and myocardial perfusion in asymptomatic adults (from the Multi-Ethnic Study of Atherosclerosis [MESA]). Am J Cardiol 2015;115:1341-5.

36. von Scholten BJ, Hasbak P, Christensen TE, Ghotbi AA, Kjaer A, Rossing P, Hansen TW. Cardiac (82)Rb PET/CT for fast and non-invasive assessment of microvascular function and structure in asymptomatic patients with type 2 diabetes. Diabetologia 2016;59:371- 8 .

37. Zhao HJ, Wang S, Cheng H, Zhang MZ, Takahashi T, Fogo AB, et al. Endothelial nitric oxide synthase deficiency produces accelerated nephropathy in diabetic mice. J Am Soc Nephrol 2006;17:2664-9.

38. Nakagawa T, Sato W, Glushakova O, Heinig M, Clarke T, Campbell-Thompson M, et al. Diabetic endothelial nitric oxide synthase knockout mice develop advanced diabetic nephropathy. J Am Soc Nephrol 2007;18:539-50.

39. Nakagawa T. Uncoupling of the VEGF-endothelial nitric oxide axis in diabetic nephropathy: an explanation for the paradoxical effects of VEGF in renal disease. Am J Physiol Renal Physiol 2007;292:F1665-72.

40. Vora JP, Dolben J, Dean JD, Thomas D, Williams JD, Owens DR, Peters JR. Renal hemodynamics in newly presenting non-insulin dependent diabetes mellitus.Kidney Int 1992;41:829-35.

41. Freda BJ, Tang WH, Van Lente F, Peacock WF, Francis GS. Cardiac troponins in renal insufficiency: review and clinical implications. J Am Coll Cardiol 2002;40:2065-71.

42. Bansal N, Katz R, Dalrymple L, de Boer I, DeFilippi C, Kestenbaum B, et al. NT-proBNP and troponin $\mathrm{T}$ and risk of rapid kidney function decline and incident CKD in elderly adults. Clin J Am Soc Nephrol 2015; 10:205-14.

43. Ho JE, Hwang SJ, Wollert KC, Larson MG, Cheng S, Kempf T, et al. Biomarkers of cardiovascular stress and incident chronic kidney disease. Clin Chem 2013;59:1613-20. 


\section{SUPPLEMENTAL METHODS}

\section{$>$ KIDNEY FUNCTION}

The glomerular filtration rate (GFR) was estimated with the CKD-EPI equation based on the combination of serum creatinine and serum cystatin $C$ (eGFR crys) for the primary analyses. For additional analyses, eGFR was calculated with the CKD-EPI equations based on serum creatinine (eGFR $\mathrm{cr}$ ) and serum cystatin $\mathrm{C}(\mathrm{eGFR}$ cys). Serum creatinine was measured with a Jaffé method traceable to isotope dilution mass spectrometry (due to a change of supplier by the Beckman Synchron LX20, Beckman Coulter; and the Roche Cobas 6ooo, F. Hoffmann-La Roche). Serum cystatin C was measured with a particle enhanced immunoturbidimetric assay standardized against ERM-DA471/IFCC reference material (Roche Cobas 80oo, Roche).

To assess urinary albumin excretion (UAE), participants were requested to collect two $24 \mathrm{~h}$ urine collections. Urinary albumin concentration was measured with a standard immunoturbidimetric assay by an automatic analyzer (due to a change of supplier by the Beckman Synchron LX20, Beckman; and the Roche Cobas 6000, Roche) and multiplied by collection volume to obtain the $24 \mathrm{~h}$ UAE. A urinary albumin concentration below the detection limit of the assay ( $2 \mathrm{mg} / \mathrm{l}$ for the Beckman Synchron LX2O and $3 \mathrm{mg} / \mathrm{l}$ for the Roche Cobas 6000) was set at $1.5 \mathrm{mg} / \mathrm{l}$ before multiplying by collection volume. Only urine collections with a collection time between $2 \mathrm{oh}$ and $28 \mathrm{~h}$ were considered valid. If needed, UAE was extrapolated to a 24 -h excretion. UAE was preferably based on the average of two (available in $91.9 \%$ of the participants) 24-h urine collections.

\section{> FURTHER DETAILS ON, AND DEFINITIONS OF, COVARIATES}

Glucose metabolism was classified according to the World Health Organization 2006 criteria $^{1}$ into normal glucose metabolism, impaired fasting glucose, impaired glucose tolerance, and diabetes mellitus. Participants with diabetes mellitus and participants using glucose-lowering medication were considered as having type 2 diabetes (T2DM) if they had no (self-reported) type 1 or other specific type of diabetes mellitus. For the present study, impaired fasting glucose and impaired glucose tolerance were combined into prediabetes.

Total-to-HDL cholesterol ratio was calculated by dividing total cholesterol by HDL cholesterol. Hypertension was defined as an office systolic pressure $\geq 140 \mathrm{mmHg}$, an office diastolic pressure $\geq 90 \mathrm{mmHg}$ and(or) the use of antihypertensive medication. Office pulse pressure was defined as office systolic pressure minus office diastolic pressure and office mean arterial pressure as office diastolic pressure plus 0.412 times office pulse pressure. ${ }^{2}$ Similar equations were used to calculate 24 h average ambulatory pulse pressure and $24 \mathrm{~h}$ average ambulatory mean arterial pressure, respectively.

Smoking behavior was categorized into never, former, and current. Alcohol consumption was classified into no consumption, low consumption $(<7$ glasses 
per week for women and < 14 glasses per week for men) and high consumption (> 7 glasses per week for women and > 14 glasses per week for men). Educational level was classified into low (none, primary, or lower vocational education only), intermediate (intermediate general secondary, intermediate vocational or higher general secondary education) and high (higher vocational education or university level of education). ${ }^{3.4}$

Questionnaire based prevalent CVD was defined as a self-reported history of myocardial infarction, cerebrovascular infarction or hemorrhage, or percutaneous artery angioplasty of, or vascular surgery on, the coronary, abdominal, peripheral or carotid arteries. Electrocardiogram (ECG) data were coded according to the Minnesota code classification system ${ }^{5}$, and ischemic ECG abnormalities were defined as a Minnesota code 1.1.1 - 1.2.8 (major or medium Q or QS waves), 7.1.1 - 7.1.2 (complete left bundle branch block), 1.3.1-1.3.2 (minor Q or QS waves), or any of 4.1.1, 4.1.2, 4.2 and 4.3 if accompanied by 5.1, 5.2 or 5.3 (ST segment abnormalities accompanied by abnormal T waves). ${ }^{6}$ 


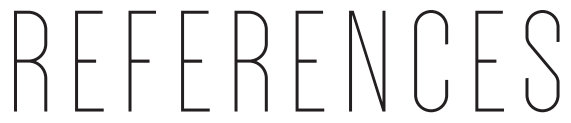

1. World Health Organization. Definition and diagnosis of diabetes mellitus and intermediate hyperglycemia: report of a WHO/IDF consultation20o6: Available from: http://www.who.int/diabetes/publications/Definition\%20and\%2odiagnosis\%20of\%20 diabetes_new.pdf.

2. Meaney E, Alva F, Moguel R, Meaney A, Alva J, Webel R. Formula and nomogram for the sphygmomanometric calculation of the mean arterial pressure. Heart. 2000 Jul;84(1):64.

3. De Bie SE. Standaardvragen 1987: Voorstellen voor uniformering van vraagstellingen naar achtergrondkenmerken en interviews [Standard questions 1987: Proposal for uniformisation of questions regarding background variables and interviews]. Leiden, The Netherlands: Leiden University Press; 1987.

4. Spauwen PJ, van Boxtel MP, Verhey FR, Kohler S, Sep SJ, Koster A, et al. Both Low and High 24-Hour Diastolic Blood Pressure Are Associated With Worse Cognitive Performance in Type 2 Diabetes: The Maastricht Study. Diabetes Care. 2015 Aug;38(8):1473-80.

5. Prineas R, Crow R, Zhang Z. The Minnesota code manual of electrocardiographic findings. 2nd ed. London: Springer-Verlag; 2010.

6. Keen H, Morrish N, Lee ET. An analysis of serial Minnesota ECG code changes in the London cohort of the WHO Multinational Study of Vascular Disease in Diabetes. Diabetologia. 2001 Sep;44 Suppl 2:S72-7. 


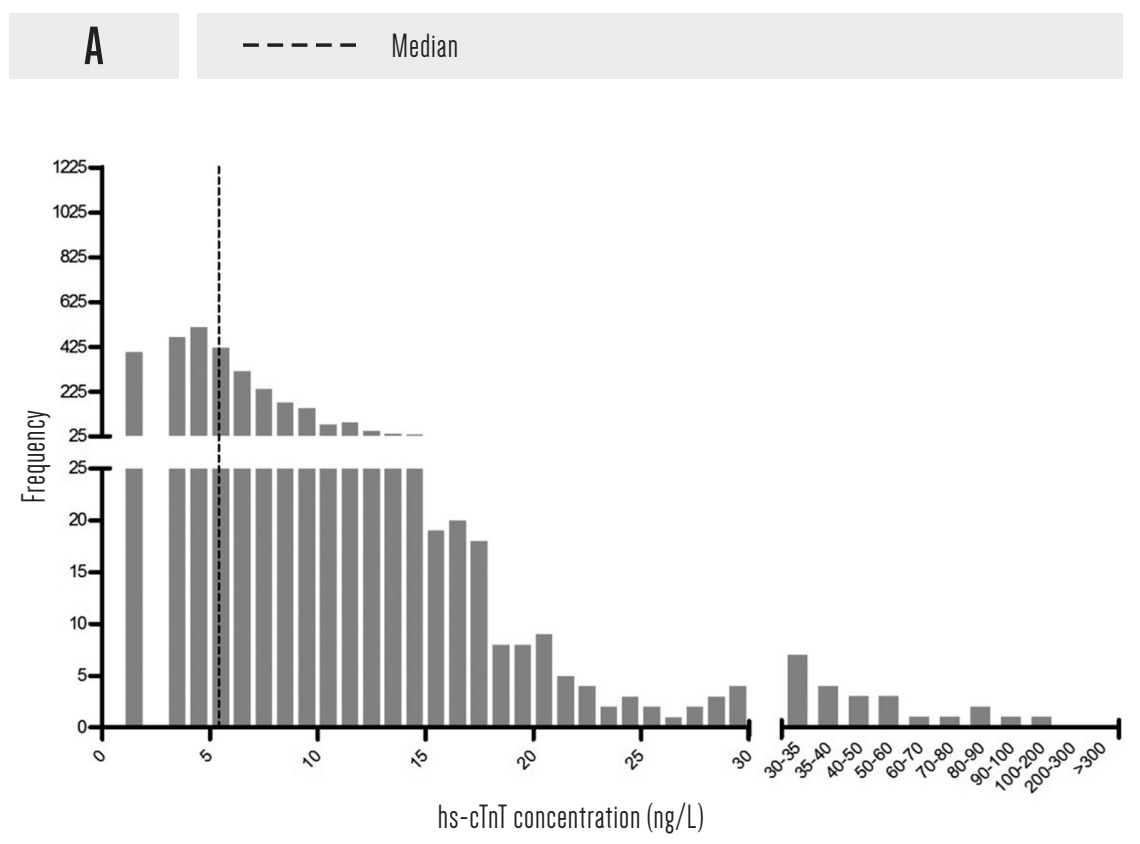

Supplemental

Figure 6.1.

Distribution of

hs-cTnT (panel A)

and hs-cTnI

(panel B).

Dashed lines

indicate median

concentrations.

\section{B $\quad----$ Median}

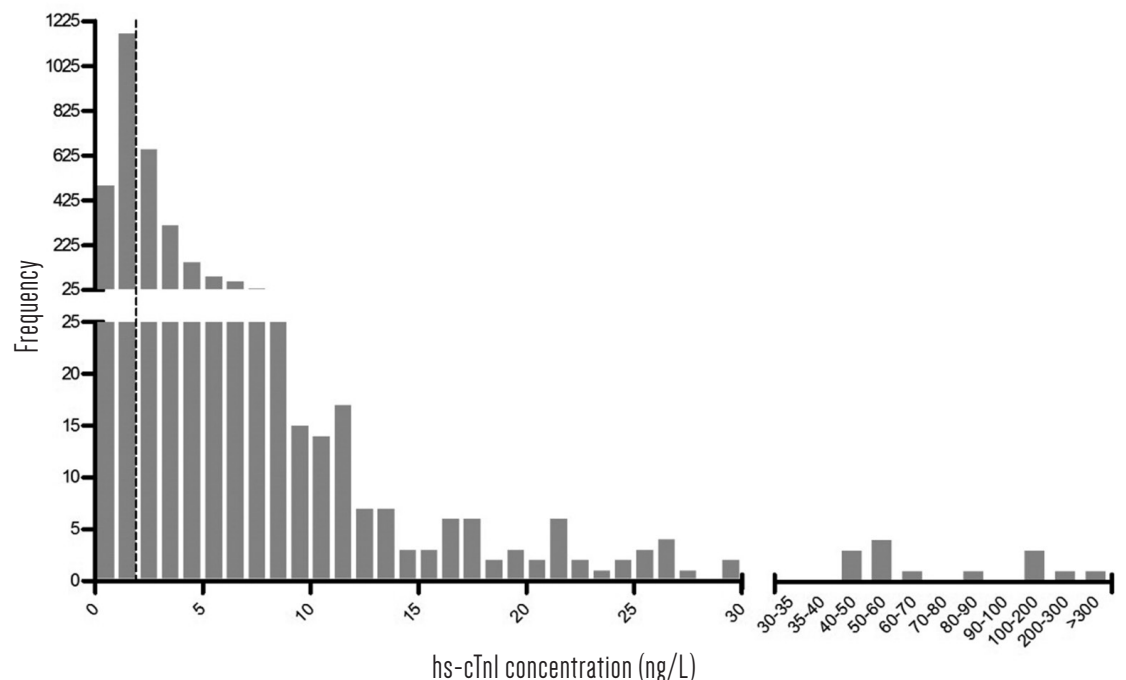

hs-cTnl concentration (ng/L) 


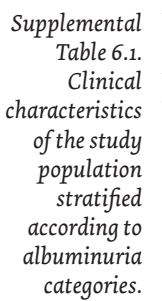

\begin{tabular}{|c|c|c|}
\hline & Study population, $n=3103$ & $\begin{array}{l}\text { Albuminuria categories } \\
<15 \mathrm{mg} / 24 \mathrm{~h}, \mathrm{n}=2514\end{array}$ \\
\hline \multicolumn{3}{|l|}{ Demographics } \\
\hline Age (years) & $59.8 \pm 8.2$ & $59.2 \pm 8.2$ \\
\hline Men & $1608(51.8 \%)$ & $1216(48.4 \%)$ \\
\hline \multicolumn{3}{|l|}{ Educational level } \\
\hline Low & $517(16.7 \%)$ & $381(15.2 \%)$ \\
\hline $\begin{array}{l}\text { Intermediate } \\
\text { High }\end{array}$ & $\begin{array}{l}327(42.8 \%) \\
1259(40.6 \%)\end{array}$ & $\begin{array}{l}1089(43.3 \%) \\
1044(415 \%)\end{array}$ \\
\hline Ischemic ECG abnormalities & $333(10.7 \%)$ & $249(9.9 \%)$ \\
\hline Questionnaire based prevalent cardiovascular disease $e^{a}$ & $504(16.5 \%)$ & $360(14.5 \%)$ \\
\hline \multicolumn{3}{|l|}{ Lifestyle variables } \\
\hline \multicolumn{3}{|l|}{ Smoking behaviour } \\
\hline $\begin{array}{l}\text { Never smoker } \\
\text { Former smoker }\end{array}$ & $\begin{array}{l}1088(35.1 \%) \\
1596(51.4 \%)\end{array}$ & $\begin{array}{l}930(37.0 \%) \\
1263(50.2 \%)\end{array}$ \\
\hline Current smoker & $419(13.5 \%)$ & $321(12.8 \%)$ \\
\hline \multicolumn{3}{|l|}{ Alcohol consumption } \\
\hline $\begin{array}{l}\text { None } \\
\text { Low (women } \leq 7 \text { glasses/week: men } \leq 14 \text { glasses/week) }\end{array}$ & $\begin{array}{l}572(18.4 \%) \\
1720(55.4 \%)\end{array}$ & $\begin{array}{l}446(17.7 \%) \\
1403(55.8 \%)\end{array}$ \\
\hline \multirow{2}{*}{\multicolumn{3}{|c|}{ Metabolic variables }} \\
\hline & & \\
\hline \multicolumn{3}{|l|}{ Waist circumference (cm) } \\
\hline Women & $89.9 \pm 13.0$ & $89.2 \pm 12.3$ \\
\hline Office systolic pressure, mmHg & $|35| \pm 18.2$. & $133.4 \pm 17.5$ \\
\hline Office diastolic pressure, mmHg & $76.2 \pm 9.9$ & $75.8 \pm 9.8$ \\
\hline 24h average ambulatory systolic pressure, mmHg ${ }^{b}$ & $\|9 . \mid \pm\| .8$ & $117.8 \pm \| 1 . .1$ \\
\hline 24h average ambulatory diastolic pressure, mmHg ${ }^{b}$ & $73.5 \pm 7.2$ & $73.2 \pm 7.0$ \\
\hline Hypertension & $1742(56.1 \%)$ & $1289(51.3 \%)$ \\
\hline \multicolumn{3}{|l|}{ Glucose metabolism status } \\
\hline Normal glucose metabolism & $1775(57.2 \%)$ & I565 (62.3\%) \\
\hline Impaired fasting glucose & $129(4.2 \%)$ & $103(4.1 \%)$ \\
\hline $\begin{array}{l}\text { Impaired glucose tolerance } \\
\text { Type } 2 \text { diabetes }\end{array}$ & $\begin{array}{l}34 \mid(11.0 \%) \\
858(277 \%)\end{array}$ & $\begin{array}{l}294(11.7 \%) \\
552(22.0 \%)\end{array}$ \\
\hline \multicolumn{3}{|l|}{ HbAIC $(\%)^{c}$} \\
\hline Without type 2 diabetes & $5.5 \pm 0.4$ & $5.5 \pm 0.4$ \\
\hline With type 2 diabetes & $6.9 \pm 1.0$ & $6.9 \pm 1.1$ \\
\hline Total cholesterol, mg/dL & $202.7 \pm 44.8$ & $205.9 \pm 43.9$ \\
\hline \multicolumn{3}{|l|}{ HDL cholesterol, mg/dL } \\
\hline Men & $51.4 \pm 14.5$ & $52.2 \pm 14.2$ \\
\hline Women & $67.2 \pm 18.5$ & $67.7 \pm 18.5$ \\
\hline LDL cholesterol, mg/dL & $119.5 \pm 40.0$ & $122.4 \pm 39.3$ \\
\hline Triglycerides, mg/dL & I07.2 [77.9-152.3] & I04.I [76.2-145.5] \\
\hline Total-to-HDL cholesterol ratio & $3.7 \pm 1.2$ & $3.7 \pm 1.2$ \\
\hline \multicolumn{3}{|l|}{ Kidney function } \\
\hline eGFR $R_{\text {orrys, }} \mathrm{mL} / \mathrm{min} / 1.73 \mathrm{~m}^{2}$ & $88.2 \pm 14.8$ & $89.1 \pm 14.0$ \\
\hline $\mathrm{eGF} \mathrm{R}_{\mathrm{Or}}, \mathrm{mL} / \mathrm{min} / .1 .7 \mathrm{~m}^{2}$ & $84.5 \pm 13.9$ & $85.0 \pm 13.4$ \\
\hline $\mathrm{eGFF} \mathrm{R}_{\mathrm{es}} \mathrm{mL} / \mathrm{min} / .73 \mathrm{~m}^{2}$ & $89.8 \pm 16.5$ & $91.1 \pm 15.5$ \\
\hline \\
\hline $\begin{array}{l}\geq 90 \mathrm{~mL} / \mathrm{min} / 1.73 \mathrm{~m}^{2} \\
60-<90 \mathrm{~mL} / \mathrm{min}^{\prime} / .73 \mathrm{~m}^{2}\end{array}$ & $\begin{array}{l}1497(48.2 \%) \\
1480(47.7 \%)\end{array}$ & $\begin{array}{l}126 \mid(50.2 \%) \\
\mid 187(47.2 \%)\end{array}$ \\
\hline $\begin{array}{l}<0 \mathrm{~mL} / \mathrm{min} / 1.73 \mathrm{~m}^{2} \\
\text { Urinary albumin excretion (mg/24h) }\end{array}$ & $\begin{array}{l}126(4.1 \%) \\
6.7[4.1-1.9]\end{array}$ & $\begin{array}{l}5.6[3.7-8.3] \\
50(2.0 \%)\end{array}$ \\
\hline \multicolumn{3}{|l|}{ Medication } \\
\hline Antihypertensive medication & $1223(39.4 \%)$ & $874(34.8 \%)$ \\
\hline Renin-angiotensin system inhibitor & $927(29.9 \%)$ & $639(25.4 \%)$ \\
\hline \multirow{2}{*}{\multicolumn{3}{|c|}{$\begin{array}{l}\text { Lipid-modifying medication } \\
\text { Cardiac biomarkers }\end{array}$}} \\
\hline & & \\
\hline hs-cTnT (ng/l) & $\begin{array}{l}6.6 \pm 6.1 \\
5.4[3.9-7.9]\end{array}$ & $\begin{array}{l}6.0 \pm 4.9 \\
5.1[3.7-7.3]\end{array}$ \\
\hline hs-cTnl (ng/l) & $\begin{array}{l}3.2 \pm 9.1 \\
19[13-31]\end{array}$ & $\begin{array}{l}2.9 \pm 8.8 \\
18[12-297\end{array}$ \\
\hline NT-proBNP (ng/l) & $\begin{array}{l}82.6 \pm 133.2 \\
50.9[29.1-90.5]\end{array}$ & $\begin{array}{l}73.4 \pm 93.3 \\
49.0[28.6-87.5]\end{array}$ \\
\hline
\end{tabular}




\begin{tabular}{|c|c|c|}
\hline $\begin{array}{l}\text { Albuminuria categories } \\
15-<30 \mathrm{mg} / 24 \mathrm{~h}, \mathrm{n}=323\end{array}$ & $\begin{array}{l}\text { Albuminuria categories } \\
\pm 30 \mathrm{mg} / 24 \mathrm{~h}, \mathrm{n}=266\end{array}$ & \\
\hline & & Pvalued \\
\hline $61.9 \pm 8.3$ & $63.0 \pm 7.6$ & $<0.001$ \\
\hline $195(60.4 \%)$ & $197(74.1 \%)$ & $<0.001$ \\
\hline $\begin{array}{l}58(18.0 \%) \\
|4|(43.7 \%) \\
124(38.4 \%)\end{array}$ & $\begin{array}{l}78(29.3 \%) \\
97(36.5 \%) \\
91(34.2 \%)\end{array}$ & $<0.001$ \\
\hline $26(8.0 \%)$ & $58(21.8 \%)$ & $<0.001$ \\
\hline $63(19.9 \%)$ & $81(30.9 \%)$ & $<0.001$ \\
\hline $\begin{array}{l}95(29.4 \%) \\
183(56.7 \%) \\
45(13.9 \%)\end{array}$ & $\begin{array}{l}63(23.7 \%) \\
150(56.4 \%) \\
53(19.9 \%)\end{array}$ & $<0.001$ \\
\hline $\begin{array}{l}61(18.9 \%) \\
178(55.1 \%) \\
84(26.0 \%)\end{array}$ & $\begin{array}{l}65(24.4 \%) \\
139(52.3 \%) \\
62(23.3 \%)\end{array}$ & 0.12 \\
\hline
\end{tabular}

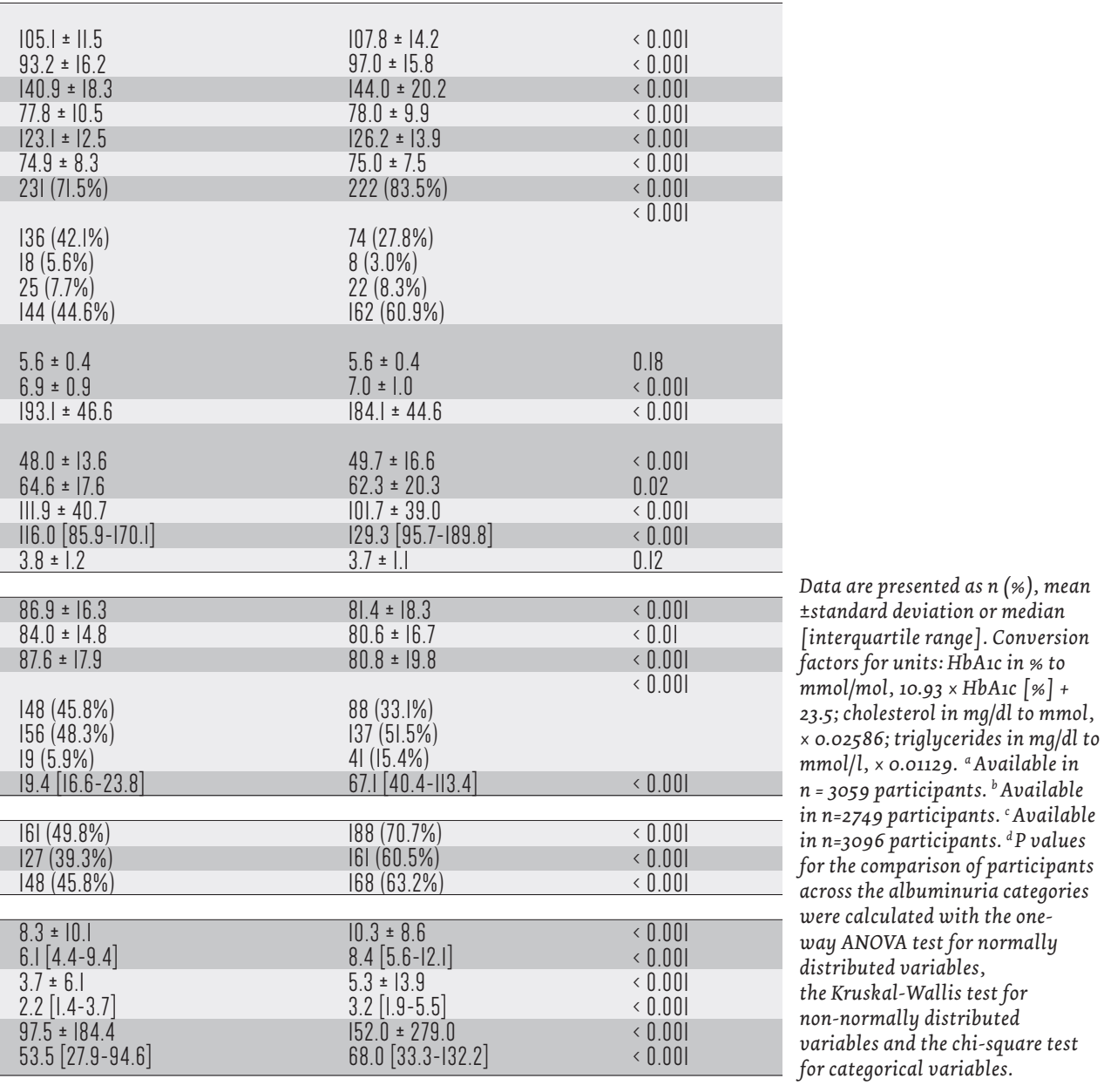




\begin{tabular}{|c|c|c|c|c|c|c|c|c|c|}
\hline \multirow{3}{*}{$\begin{array}{r}\text { Supplemental } \\
\text { Table } 6.2 . \\
\text { Associations } \\
\text { of eGFR with } \\
\text { biomarkers of } \\
\text { cardiac injury } \\
\text { using different } \\
\text { eGFR equations. }\end{array}$} & \multicolumn{3}{|c|}{ eGFR } & \multicolumn{2}{|c|}{$\geq 90 \mathrm{~mL} / \mathrm{min} / \mathrm{I} .73 \mathrm{~m}^{2}$} & \multicolumn{2}{|c|}{60 to $<90 \mathrm{~mL} / \mathrm{min} / \mathrm{l} .73 \mathrm{~m}^{2}$} & \multicolumn{2}{|c|}{$<60 \mathrm{~mL} / \mathrm{min} / \mathrm{l} .73 \mathrm{~m}^{2}$} \\
\hline & Biomarker & eGFR equation & Model & Ratio (95\% CI) & Pvalue & Ratio (95\% Cl) & Pvalue & Ratio (95\% Cl) & Pvalue \\
\hline & hs-cTnT & $e G F R_{\text {or }}$ & $4 a$ & Reference & $\mathrm{n} / \mathrm{a}$ & 1.14 (1.10; I.19) & $<0.001$ & I.47 (I.35; I.6I) & $<0.001$ \\
\hline & & $\mathrm{eGFR}_{\text {cys }}$ & $4 a$ & Reference & $\mathrm{n} / \mathrm{a}$ & I.I7 (I.I3; I.22) & $<0.001$ & $1.67(1.53 ; 1.82)$ & $<0.001$ \\
\hline & & $e G R_{\text {croys }}$ & $4 a$ & Reference & n/a & I.21 (I.17; I.26) & $<0.001$ & $1.69(1.53 ; 1.85)$ & $<0.001$ \\
\hline & hs-cInl & $\mathrm{eGFR}_{\mathrm{or}}$ & $4 a$ & Reference & n/a & I.II (I.05; I.17) & $<0.001$ & 1.39 (I.22; 1.57) & $<0.001$ \\
\hline & & eGFR $\mathrm{Rys}_{\text {cys }}$ & $4 a$ & Reference & n/a & $1.12(1.05 ; 1.18)$ & $<0.001$ & $1.40(1.23 ; 1.60)$ & $<0.001$ \\
\hline & & $e G F R_{\text {croys }}$ & $4 a$ & Reference & n/a & $1.14(1.07 ; 1.20)$ & $<0.001$ & I.45 (I.26; I.66) & $<0.001$ \\
\hline & NT-proBNF & eGFR & $4 a$ & Reference & n/a & I.II (I.04; I.19) & $<0.001$ & I.45 (I.24; I.69) & $<0.001$ \\
\hline & & $e_{G F R_{\text {cys }}}$ & $4 a$ & Reference & n/a & $1.17(1.10 ; 1.26)$ & $<0.001$ & $1.97(1.69 ; 2.30)$ & $<0.001$ \\
\hline & & $e \mathrm{GF}_{\text {crcys }}$ & $4 a$ & Reference & $\mathrm{n} / \mathrm{a}$ & I.19 (I.12; I.27) & $<0.001$ & $1.94(1.64 ; 2.29)$ & $<0.001$ \\
\hline
\end{tabular}

Betas represent the ratio of geometric mean concentrations of cardiac biomarkers in the respective eGFR crys category relative to participants with an eGFRcrys $\geq 90 \mathrm{ml} / \mathrm{min} / 1.73 \mathrm{~m}^{2}$. Model 4 a: age, sex, glucose metabolism status, waist circumference, total-to-HDL cholesterol ratio, triglycerides, use of lipid-modifying medication, smoking behavior, alcohol consumption, educational level, UAE (categorical), office systolic blood pressure, use of antihypertensive medication, ECG based prior cardiac ischemia. 


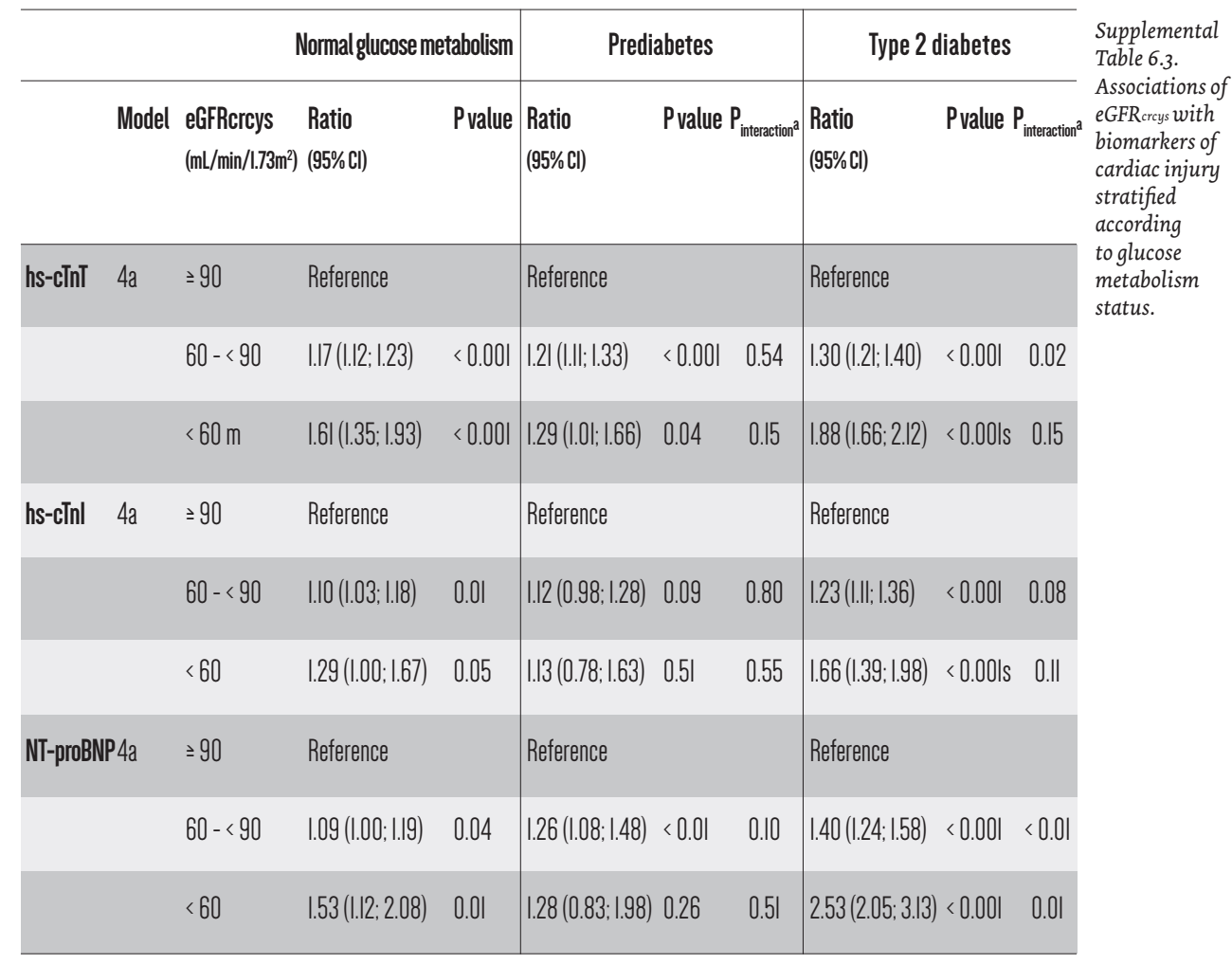

Betas represent the ratio of geometric mean concentrations of cardiac biomarkers in the respective eGFR crys category relative to participants with an eGFR creys $\geq 90 \mathrm{ml} / \mathrm{min} / 1.73 \mathrm{~m}^{2}$. Model $4 a$ : age, sex, waist circumference, total-to-HDL cholesterol ratio, triglycerides, use of lipid-modifying medication, smoking behavior, alcohol consumption, educational level, UAE (categorical), office systolic pressure, use of antihypertensive medication, ischemic ECG abnormalities. ${ }^{a} P_{\text {interaction }}$ as compared with participants with normal glucose metabolism. 


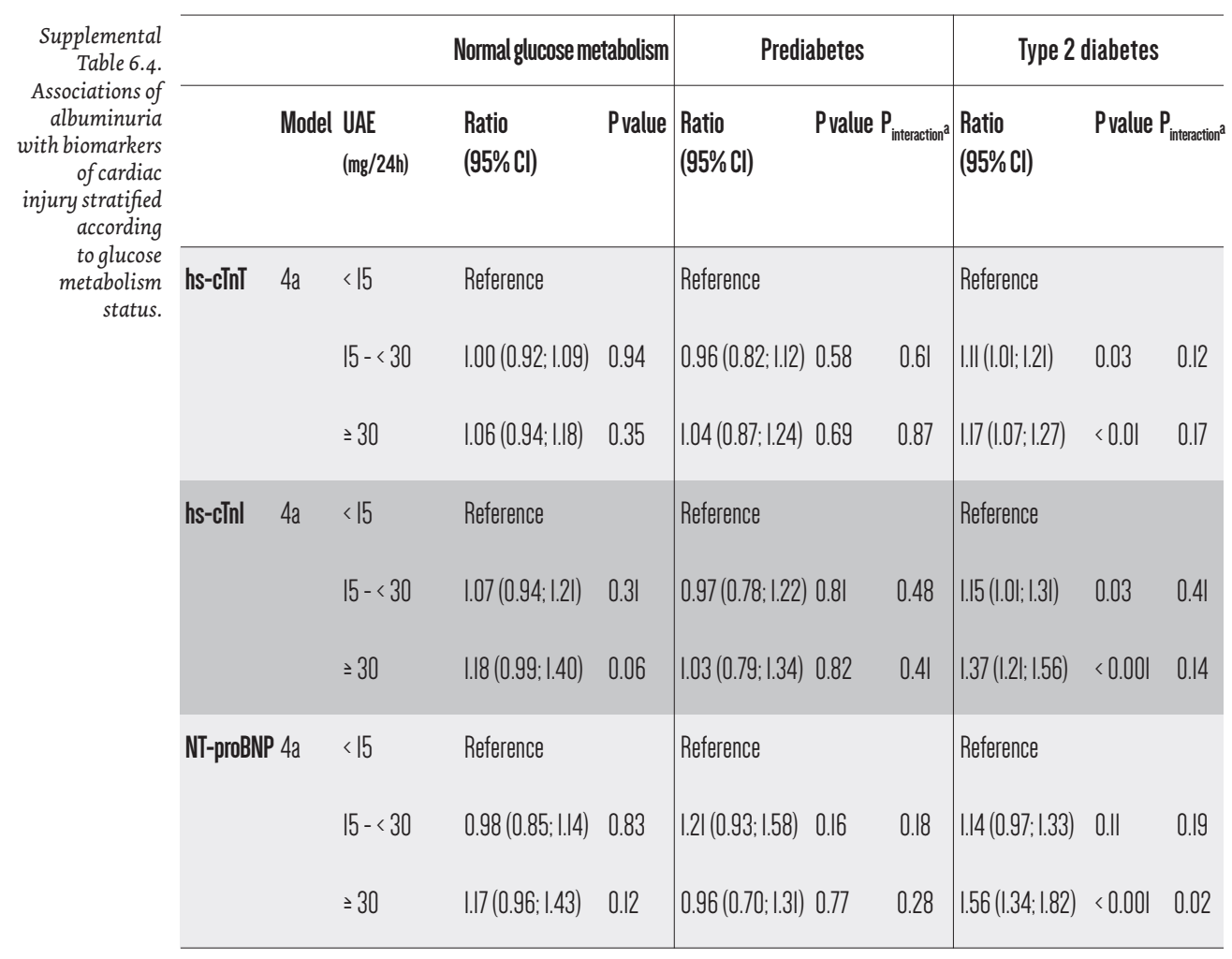

Betas represent the ratio of geometric mean concentrations of cardiac biomarkers in the respective albuminuria category relative to participants with a UAE $<15 \mathrm{mg} / 24 \mathrm{~h}$. Model $4 a$ : age, sex, waist circumference, total-to-HDL cholesterol ratio, triglycerides, use of lipid-modifying medication, smoking behavior, alcohol consumption, educational level, eGFR creys (categorical), office systolic pressure, use of antihypertensive medication, ischemic ECG abnormalities. ${ }^{a} P_{\text {interaction }}$ as compared with participants with normal glucose metabolism. 


\begin{tabular}{|c|c|c|c|c|c|c|}
\hline \multirow[b]{2}{*}{ Model } & \multirow[b]{2}{*}{$\begin{array}{l}\text { eGFR } \\
\left(\mathrm{mL} / \mathrm{min} / \mathrm{l} .73 \mathrm{~m}^{2}\right)\end{array}$} & \multicolumn{2}{|c|}{ Ischemic ECG abnormalities } & \multirow[b]{2}{*}{$\begin{array}{l}\text { UAE } \\
\text { (mg/24h) }\end{array}$} & \multicolumn{2}{|c|}{ Ischemic ECG abnormalities } \\
\hline & & OR (95\% Cl) & Pvalue & & OR $(95 \%$ Cl) & Pvalue \\
\hline \multirow[t]{3}{*}{ I } & $\geq 90$ & Reference & & $<15$ & Reference & \\
\hline & $60-<90$ & $1.07(0.84 ; 1.35)$ & 0.60 & $15-<30$ & $0.80(0.52 ; 1.21)$ & 0.29 \\
\hline & $<60$ & $2.34(1.47 ; 3.71)$ & $<0.001$ & $\geq 30$ & $2.54(1.84 ; 3.49)$ & $<0.001$ \\
\hline \multirow[t]{3}{*}{2} & $\geq 90$ & Reference & & $<15$ & Reference & \\
\hline & $60-<90$ & $0.85(0.66 ; 1.10)$ & 0.21 & $15-<30$ & $0.64(0.42 ; 0.99)$ & 0.04 \\
\hline & $<60$ & $1.37(0.84 ; 2.25)$ & 0.21 & $\geq 30$ & $1.85(1.32 ; 2.59)$ & $<0.001$ \\
\hline \multirow[t]{3}{*}{3} & $\geq 90$ & Reference & & $<15$ & Reference & \\
\hline & $60-<90$ & $0.81(0.62 ; 1.05)$ & 0.10 & $15-<30$ & $0.61(0.39 ; 0.93)$ & 0.02 \\
\hline & $<60$ & $1.15(0.69 ; 1.92)$ & 0.59 & $\geq 30$ & $1.74(1.23 ; 2.48)$ & $<0.01$ \\
\hline \multirow[t]{3}{*}{$4 a$} & $\geq 90$ & Reference & & $<15$ & Reference & \\
\hline & $60-<90$ & 0.80 (0.61; I.03) & 0.09 & $15-<30$ & 0.60 (0.39; 0.92$)$ & 0.02 \\
\hline & $<60$ & $1.07(0.64 ; 1.79)$ & 0.80 & $\geq 30$ & I.68 (I.I8; 2.39) & $<0.01$ \\
\hline \multirow[t]{3}{*}{$4 b$} & $\geq 90$ & Reference & & $<15$ & Reference & \\
\hline & $60-<90$ & $0.80(0.61 ; 1.06)$ & 0.12 & $15-<30$ & $0.60(0.38 ; 0.95)$ & 0.03 \\
\hline & $<60$ & $1.15(0.67 ; 1.98)$ & 0.61 & $\geq 30$ & $1.58(1.08 ; 2.30)$ & 0.02 \\
\hline
\end{tabular}

Supplemental Table 6.5. Associations of eGFR crrys $_{\text {and }}$ and albuminuria with ischemic ECG abnormalities.

Odds ratios represent the odds ratio for the presence of ischemic ECG abnormalities relative to the odds in participants with an eGFR crys $\geq 90 \mathrm{ml} / \mathrm{min} / 1.73 \mathrm{~m}^{2}$ and relative to the odds in participants with a UAE $<15 \mathrm{mg} / 24 \mathrm{~h}$, respectively. Model 1: unadjusted model; Model 2: age, sex, glucose metabolism status; Model 3: model $2+$ waist circumference, total-to-HDL cholesterol ratio, triglycerides, use of lipid-modifying medication, smoking behavior, alcohol consumption, educational level, UAE (categorical)/eGFR crys (categorical); model 4a: office systolic pressure, use of antihypertensive medication; Model $4 b$ : similar to model 4 a but adjusted for 24 h average ambulatory systolic pressure instead of office systolic pressure (missing in $n=354$ participants). 


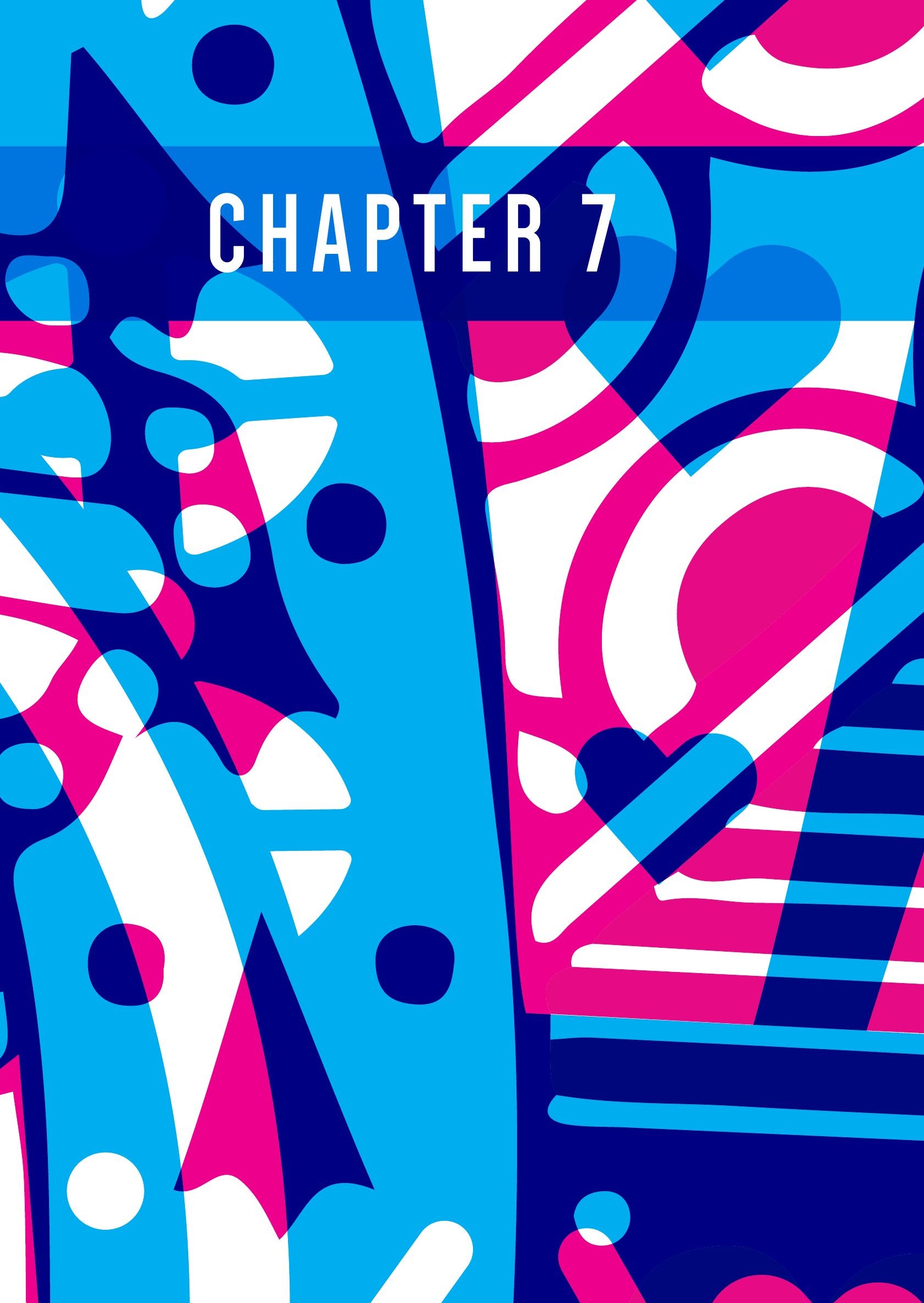




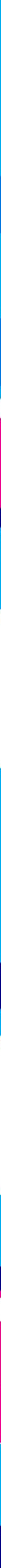




\section{CHAPTER 7}

GENERAL DISCUSSION 


\section{MAIN FINDINGS OF THIS THESIS}

Two main topics associated with the use of high-sensitivity cardiac troponin I (hscTnI) and T (hs-cTnT) assays were investigated: female versus male hearts, and battle of the cardiac troponins. The key findings of this thesis are:

1. Female-specific $99^{\text {th }}$ percentile upper reference limits (URLs) of hs-cTnI and hs-cTnT are substantially lower than male-specific $99^{\text {th }}$ percentile URLs, in particular for hs-cTnI (CHAPTER 2 AND 3).

2. The hs-cTnI distribution is more profoundly affected by extreme outliers than the hs-cTnT distribution (CHAPTER 2), and the statistical approach rather than reference population heterogeneity contributes to current discordant clinical decision limits of hs-cTnI and hs-cTnT (CHAPTER 3).

3. As compared with the acute cardiac care setting, there is a poor correlation and limited concordance between hs-cTnI and hs-cTnT in the non-acute setting (CHAPTER 5).

\section{FEMALE-SPECIFIC 99TH PERCENTILE URLS OF HS-CTNI AND HS-CTNT ARE SUBSTANTIALLY LOWER THAN MALE-SPECIFIC 99TH PERCENTILE URLS, IN PARTICULAR FOR HS-CTNI}

Prognosis for women after acute myocardial infarction (AMI) is worse than for men. A number of factors may underlie this prognostic gap between women and men: 1) The pathobiology of coronary artery diseases (CAD) is different between sexes. Women have less obstructive pericardial CAD than men, but present more frequently with non-obstructive CAD. ${ }^{1-3}$ Thrombus formation and plaque erosion is more prevalent in women than men. ${ }^{4}$ Another contributing factor could be that the microvascular system of women seems to be more affected as compared with men. ${ }^{5}$ Endogenous estrogens levels seems to provide protection for development of CAD in premenopausal women. ${ }^{6}$ Although the underlying mechanisms are not fully understood, and mainly hypothesis-based at this point, estrogen seems to attenuate the processes of atherosclerosis, left ventricular hypertrophy (LVH) and cardiomyocyte apoptosis. ${ }^{78}$ It is hypothesized that disappearance of the cardio 
protective estrogen effect after menopause results in high-risk women with faster development of CAD. This phenomenon may explain the older age at presentation of women with AMI. It is therefore of crucial importance to gain more knowledge on the development of coronary vascular diseases (CVD) in women in general and the influence of hormones on this process, to identify the "high-riskwomen". 2) Treatment by male physicians instead of treatment by female physicians results into a higher mortality rate for women after AMI, and might thus also partly contribute to the disadvantage women have when they are presenting at the emergency department. ${ }^{9}$ This striking effect became less prominent when male physicians had more experience in treating women suspected with AMI, and when they worked together with female physicians. ${ }^{9}$ Future work should establish the influence of this gender issue and coordination between health care professionals and survival of women after AMI. 3) Women tend to receive less aggressive care than men after AMI diagnosis. This includes evidence-based medications as well as invasive procedures, even if these therapies have been shown with a similar effect in women versus men..$^{10-12} 4$ ) In the past, studies not always enrolled a sufficient number of females, and thereby, sex-stratified analysis were mostly not conducted. The underrepresentation of women in research may have led to the result that some recommended diagnostic and therapeutic approaches for AMI are partly based on a "men-design", and possibly not (entirely) suitable for women. ${ }^{10} 5$ ) Last but not least, the diagnostic algorithm may not be sensitive enough to detect all AMIs in women. The current diagnostic algorithm may require an additional microvascular approach to prevent underdiagnosis of AMI in the subgroup of individuals with microvascular coronary dysfunction, which comprises particularly women. In addition, presentation of AMI in women seems an important contributor which results into under- and misdiagnosis of AMI. ${ }^{13-16}$ For a long period it was assumed that the typical signs for having an AMI are equal between sexes. To date, we know that typical symptoms are less likely presented in women than men..$^{13-16}$ Hence, determination of the typical clinical presentation of women with AMI should be the subject of future research.

We investigated whether lower cardiac troponin levels in women than men translate into different $99^{\text {th }}$ percentile values. Our studies demonstrate that the effect of sex differences on $99^{\text {th }}$ percentiles of hs-cTn is evident. Cardiac troponin levels in healthy women are lower than in healthy men and resulted into lower female-specific $99^{\text {th }}$ URLs than male-specific $99^{\text {th }}$ URLs of hs-cTnI and hs-cTnT (CHAPTER 2). We not only reveal differences between sex-specific cutoffs, but additionally, we show that female-specific $99^{\text {th }}$ percentile URLs of hs-cTnI and hs-cTnT are lower than the commonly used package insert overall $99^{\text {th }}$ percentile URLs of $26 \mathrm{ng} / \mathrm{L}$ and $14 \mathrm{ng} / \mathrm{L}$ for hs-cTnI and hs-cTnT, respectively (CHAPTER 3). Male-specific $99^{\text {th }}$ percentiles of hscTn are more in line with overall package insert hs-cTnT thresholds. This findings supports the hypothesis that overall clinical decision limits may be too high for women, hence contributing to underdiagnosis of AMI in women. Future clinical work investigating sex-specific versus overall cutoffs in relation to (under)diagnosis of AMI is therefore urgently needed. 
The sex effect seems stronger for hs-cTnI than hs-cTnT; approximately $90 \%$ of hs-cTnI studies versus $60 \%$ of hs-cTnT studies showed lower female-specific $99^{\text {th }}$ percentiles vales as compared with recommended overall thresholds. Although this phenomenon has not been clarified yet, the stronger sex effect for hs-cTnI than hscTnT seems to be confirmed in the results of clinical studies who investigated sexspecific thresholds (CHAPTER 4). The effect of sex-specific thresholds for hs-cTnT on the reclassification of AMI is small. ${ }^{17}$ Conversely, the use of sex-specific thresholds for hs-cTnI resulted into a higher proportion of women with AMI, equalizing the prevalence of AMI diagnosis in women and men. ${ }^{18}$ However, this observation is not confirmed in a retrospective analysis by Trambas et al. ${ }^{19}$ Furthermore, the HighSensitivity Troponin in the Evaluation of patients with suspected Acute Coronary Syndrome (High-STEACS) trial investigated the introduction of hs-cTnI assay using sex-specific $99^{\text {th }}$ percentile values as compared with contemporary assay in relation to reclassification rate of AMI and CVD related deaths at one year. ${ }^{29}$ Reclassified individuals were twice as likely to be women ( $84 \%$ versus $41 \%)$ as compared with identified individuals with contemporary assay. A noteworthy proportion of $17 \%$ $(n=1771)$ of individuals were reclassified with hs-cTnI assay, however, this did not lead to an decrease in subsequent AMIs or CVD related deaths at one year. ${ }^{29}$

It should be noted that there are differences on the endorsement of sex-specific $99^{\text {th }}$ percentile values of hs-cTn between Europe and United States (US). In Europe, the hs-cTn assays were introduced around 2012, however, there was no attention of applying sex-specific thresholds during their implementation. Also the European manufactures of hs-cTn assays inconsistently report sex-specific cutoffs, e.g. hscTnI of Abbott do report sex-specific $99^{\text {th }}$ percentile URLs, while hs-cTnT of Roche do not. At the time the 2015 ESC guidelines were adopted, the available clinical evidence that sex-specific $99^{\text {th }}$ percentiles outperforms the overall $99^{\text {th }}$ percentiles of hs-cTn for AMI diagnosis was lacking. As a result, most clinics in Europe are using overall hs-cTn thresholds. The introduction of hs-cTn assays has recently started in the US after the Food and Drug Administration (FDA) approval of the first hscTn assay (Roche, hs-cTnT) in January 2017. At this point also two hs-cTnI assays (Beckman Coulter, Siemens) were cleared by the FDA. The US FDA approved sexspecific thresholds for hs-cTnT (female: $14 \mathrm{ng} / \mathrm{L}$ male: $22 \mathrm{ng} / \mathrm{L}$ ) in addition to overall thresholds for hs-cTnT (overall: $19 \mathrm{ng} / \mathrm{L}$ ), and the AACC/ IFCC endorsed this sexspecific cutoffs of hs-cTn for diagnosis of AMI. ${ }^{20}$ The Fourth Universal Definition of Myocardial Infarction recommends sex-specific hs-cTn cutoffs for AMI diagnosis. ${ }^{21}$ However, this expert consensus document also acknowledge the controversy that still remains of the sex-based hs-cTn approach in relation to clinical outcome. ${ }^{21}$

At this point, we feel there is not enough clinical evidence to make firm conclusions on whether it is useful to apply sex-specific hs-cTnI and/or hs-cTnT thresholds for diagnosis of AMI (CHAPTER 4). A number of considerations should be taken into account for a carefully weighted decision on the use of sex-specific hs-cTn algorithm for AMI diagnosis. The underlying mechanisms that results into lower 
hs-cTn levels for women than men are still hypothesis-based and should be further explored. Thereby, most studies enrolled only participants with the typical clinical presentation of AMI, e.g. chest pain. Since women not always present with the typical clinical presentation, a broader inclusion range for future studies and pragmatic "real-life" studies are urgently recommended. ${ }^{22,23}$

\section{THE HS-CTNI DISTRIBUTION IS MORE PROFOUNDLY AFFECTED BY EXTREME OUTLIERS THAN THE HS-CTNT DISTRIBUTION, AND THE STATISTICAL APPROACH RATHER THAN REFERENCE POPULATION HETEROGENEITY CONTRIBUTES TO CURRENT DISCORDANT CLINICAL DECISION LIMITS OF HS-CTNI AND HS-CTNT}

The transition from contemporary assays to hs-cTn assays was associated with improved diagnostic sensitivity for diagnosis of AMI. ${ }^{24-27}$ The main advantage of the more sensitive hs-cTn assays above contemporary assays is that the time frame of serial cardiac troponin measurements became significantly shorter (from 6 - 9 hours to $\leq 3$ hours) ${ }^{28}$

Interestingly, the $99^{\text {th }}$ percentile URL as diagnostic threshold is not a general rule of thumb in laboratory medicine, and the $97.5^{\text {th }}$ percentile URL is in fact a more commonly used threshold in clinical chemical practice. The $99^{\text {th }}$ percentile value of a reference population for hs-cTn is most likely pragmatically chosen by the analytical properties of the cardiac troponin assays available at that time. To ensure that observed changes stays within marginal errors ( $\leq 10 \% \mathrm{CV}$ threshold), and thus to avoid misclassification of AMI, $99^{\text {th }}$ percentile URLs instead of $97.5^{\text {th }}$ percentile URLs were chosen. Hence, the choice for $99^{\text {th }}$ percentile URLs as thresholds for AMI diagnosis is particularly based on hypothetical grounds. Apart from o/3 h algorithm, the 2015 ESC guidelines recommends an $\mathrm{o} / 1 \mathrm{~h}$ algorithm and $\mathrm{o} / 2 \mathrm{~h}$ algorithm as alternative. ${ }^{24}$ This so-called accelerated diagnostic protocols (ADP) already goes beyond the $99^{\text {th }}$ percentile URLs of hs-cTn from healthy reference population with lower cutoffs than $99^{\text {th }}$ percentile values and by using hs-cTn assay-specific delta changes. It should be noted that investigating new hs-cTn strategies is (still) challenging, as the reference test remains rather a statistical approach than a "true biological gold" reference standard, as cardiac troponin is incorporated in the diagnosis of AMI and incorporation bias is thus hardly to avoid. We revealed that hs-cTnI distribution is more affected by outliers than hs-cTnT 
distribution. A larger variation of $99^{\text {th }}$ percentile URLs is observed for hs-cTnI reference studies than for hs-cTnT reference studies (CHAPTER 3). Additionally, our data shows that handling of outliers, rather than population heterogeneity, play a key role in determination of an appropriate $99^{\text {th }}$ percentile URL. An absolute minimum requirement is a sufficient number of healthy reference individuals. ${ }^{22} \mathrm{~A}$ small sample size in combination with outliers leads to a large variation around the estimated $99^{\text {th }}$ percentile URLs. ${ }^{22,30}$ This issue is probably on the basis of large variation across studies that estimated the $99^{\text {th }}$ percentile URL, particular for hs-cTnI. Sandoval and Apple reported criteria for defining a healthy reference population and the required statistical approach for determining $99^{\text {th }}$ percentile values of hs-cTn. ${ }^{31}$ However, overall package insert $99^{\text {th }}$ percentile URLs of $26 \mathrm{ng} / \mathrm{L}$ for hs-cTnI and $14 \mathrm{ng} / \mathrm{L}$ for hs-cTnT are determined before this recommendation and vary highly in the way how they are derived from different "healthy" reference populations and differ on their conducted statistical approach. In addition, in clinical practice a number of laboratories determine their own $99^{\text {th }}$ percentile URL of hs-cTn, as the guidelines dictates to calculate $99^{\text {th }}$ percentile values with an appropriate quality control in each laboratory. ${ }^{25}$ Although this sounds theoretically valid, a sufficient number of reference individuals is often not reached. In an opinion paper we therefore recently recommended laboratories to adapt $99^{\text {th }}$ percentile URLs of hs-cTn from sufficiently large peer-reviewed studies, rather than stimulate institutional laboratories to calculate their own $99^{\text {th }}$ percentile URLs. ${ }^{32}$

The higher number of extreme outliers for hs-cTnI than hs-cTnT is not understood. We observed more hs-cTnI outliers in men than women derived from a single reference cohort, and also other hs-cTnI reference studies showed more dispersion in hs-cTnI range for men than women (CHAPTER 3, absolute difference $99^{\text {th }}$ percentile hs-cTnT-hs-cTnI: women, $-1 \mathrm{ng} / \mathrm{L}$ to $13 \mathrm{ng} / \mathrm{L}$ versus men, $4 \mathrm{ng} / \mathrm{L}$ to $30 \mathrm{ng} / \mathrm{L}$ ). Whether these extreme troponin values represent true (subclinical) cardiac pathology, rather due to imperfect assay specificity or any interference is at present unknown..$^{33-37}$

We found a substantially lower overall $99^{\text {th }}$ percentile URL for hs-cTnI of $13 \mathrm{ng} / \mathrm{L}$ from our single reference study as compared with the package insert hs-cTnI clinical decision limit of $26 \mathrm{ng} / \mathrm{L}$ (CHAPTER 2). Our systematic review confirmed that the clinically applied overall hs-cTnI cut-off of $26 \mathrm{ng} / \mathrm{L}$ does not align with the much lower cutoff values from individual reference studies (CHAPTER 3). Hence, the overall clinical decision limit of $26 \mathrm{ng} / \mathrm{L}$ for hs-cTnI may also be part of the underdiagnosis problem in women. A number of hs-cTnI studies supports the hypothesis that the clinical applied overall cut-off for hs-cTnI is too high, ${ }^{88,39}$ and as women have lower hs-cTn levels than men, this issue may be even more problematic for women than for men. Wildi et al. showed that the package insert values of $26 \mathrm{ng} / \mathrm{L}$ for hs-cTnI and $14 \mathrm{ng} / \mathrm{L}$ for hs-cTnT resulted into reclassification of AMI diagnosis in one out of five patients, and suggested to lower the hs-cTnI threshold to $9 \mathrm{ng} / \mathrm{L} \cdot .^{38}$ Recently, a retrospective study obtained similar findings, and suggested also downward adjustment of hs-cTnI threshold to $9 \mathrm{ng} / \mathrm{L}$ that lead to more bio- 
equivalent hs-cTnI-hs-cTnT thresholds. ${ }^{39,40}$ Besides investigation of sex-specific hs-cTnI thresholds, downward adjustment of overall hs-cTnI threshold of $26 \mathrm{ng} / \mathrm{L}$ should be considered (CHAPTER 2 AND 3).

\section{AS COMPARED WITH THE ACUTE CARDIAC CARE SETTING, THERE IS A POOR CORRELATION AND LIMITED CONCORDANCE BETWEEN HS-CTNI AND HS-CTNT IN THE NON-ACUTE SETTING}

As compared with the acute cardiac care setting, we observed a poor correlation and limited concordance between the hs-cTnI and hs-cTnT assays in the non-acute setting. In CHAPTER 5, we investigated in a non-acute setting to which extent signs of cardiac ischemia measured by ECG were associated with hs-cTnI and hscTnT levels. We excluded participants with self-reported history of AMI to reveal a population without substantial clinically cardiac damage. Our study shows that isolated high troponin I levels, but not isolated high troponin T levels, are independently associated with ECG abnormalities indicative of cardiac ischemia. This data suggests that associations of hs-cTnI and hs-cTnT with ECG abnormalities indicative of ischemia are driven by different mechanisms. We selected ECG criteria who are likely to reflects ischemia, but a further specification by this analysis was not possible. Furthermore, we were not able to confirm the registered cardiac ischemia by ECG with imaging modalities such as coronary imaging and echocardiography in our study. Although this ECG criteria indicates ischemia, also other mechanisms like left ventricular hypertrophy, dilated cardiomyopathy or amyloid cardiomyopathy could underlie these ECG abnormalities. Based on our results, we recommend future in vivo studies for in-depth investigation on mechanistic differences of hs-cTnI and hs-cTnT release induced by ischemia. The poor hs-cTnI-hs-cTnT correlation in non-acute setting as compared with the acute setting, ${ }^{42-45}$ may result in clinically relevant differences on prognostic performance of hs-cTnI and hs-cTn in the general population (CHAPTER 5). Future longitudinal cohort studies studying the prognostic performance of hs-cTnI and hs-cTnI in addition to established risk scores should confirm whether the discordance between hs-cTnI and hs-cTnI also translates into different prognostication performance.

Another factor that was part of the ongoing discussion over the years on hs-cTn elevations was the role of the kidney. In chronic kidney disease (CKD) patients persistently elevated cardiac troponin levels are shown, ${ }^{41,46-48}$ particularly hs-cTnT seems to be affected by reduced kidney function. ${ }^{45}$ We tried to identify whether the relation of kidney function, distinguished by estimated glomerular filtration rate (eGFR) and albuminuria, with hs-cTnI, hs-cTnT and NT-proBNP concentrations was 
already presented in individuals who did not fulfill the CKD criteria (CHAPTER 6). Both eGFR and albuminuria were independently associated with hs-cTnI, hs-cTnT and NT-proBNP levels in a population based cohort. While the associations of albuminuria with hs-cTnI and hs-cTnT were similar, a slightly stronger association is observed for eGFR with hs-cTnT than hs-cTnI. This divergence might be the result of differences in cardiac injury or renal elimination, however, we were not able to determine the relative contributions of cardiac injury versus impaired kidney function to this relationship. Recent research investigating to which extent increased hs-cTn levels are caused by cardiac injury and/or impaired renal elimination, provided evidence that impaired renal elimination is not the main driver of chronically elevated cardiac troponin T levels. ${ }^{49}$

\section{CONCLUDING REMARKS AND FUTURE DIRECTIONS}

This thesis focused on sex differences in relation to the use of diagnostic algorithms for AMI. Traditionally, and by consensus definition, the $99^{\text {th }}$ percentile URL of cardiac troponin has a central role in the diagnosis of AMI. Our studies show substantial differences between sexes in respect to their $99^{\text {th }}$ percentile URLs, which are not reflected in current clinical practice worldwide. Nevertheless, the clinical benefit of adapting sex-specific thresholds as the new standard is not yet clear cut. Increased sensitivity of hs-cTn assays triggered the development and adaption of accelerated diagnostic protocols that rely on absolute delta change values between serial hs-cTn measurements irrespective of the $99^{\text {th }}$ percentile URL. Nevertheless, also with the development of these novel protocols, sex-stratified analyses remain warranted to ensure optimized cardiac care for women and men. 
REFERENCES

1. Madonis SM, Skelding KA, Roberts M. Management of acute coronary syndromes: special considerations in women. Heart 2017;103(20):1638-46. doi: 10.1136/heartjnl-2016-309938

2. Eggers KM, Lindahl B. Impact of Sex on Cardiac Troponin Concentrations-A Critical Appraisal. Clinical chemistry 2017;63(9):1457-64. doi: 10.1373/clinchem.2017.271684

3. Bugiardini R, Bairey Merz CN. Angina with "normal" coronary arteries: a changing philosophy. JAMA 2005;293(4):477-84. doi: 10.1001/jama.293.4.477 [published Online First: 2005/01/27]

4. Pepine CJ, Ferdinand KC, Shaw LJ, et al. Emergence of Nonobstructive Coronary Artery Disease: A Woman's Problem and Need for Change in Definition on Angiography. Journal of the American College of Cardiology 2015;66(17):1918-33. doi: 10.1016/j.jacc.2015.08.876 [published Online First: 2015/10/27]

5. Appelman Y. Microvascular disease, what little we know. EuroIntervention 2018;14(5):e499-e501. doi: 10.4244/EIJV14I5A89 [published Online First: 2018/08/08]

6. Westerman S, Wenger NK. Women and heart disease, the underrecognized burden: sex differences, biases, and unmet clinical and research challenges. Clin Sci (Lond) 2016;130(8):551-63. doi: 10.1042/CS20150586

7. Donaldson C, Eder S, Baker C, et al. Estrogen attenuates left ventricular and cardiomyocyte hypertrophy by an estrogen receptor-dependent pathway that increases calcineurin degradation. Circ Res 2009;104(2):265-75, 11p following 75. doi: 10.1161/ CIRCRESAHA.108.190397

8. PiroM,Della Bona R, AbbateA, et al.Sex-related differences in myocardialremodeling. Journal of the American College of Cardiology 2010;55(11):1057-65. doi: 10.1016/j.jacc.2009.09.065

9. Greenwood BN, Carnahan S, Huang L. Patient-physician gender concordance and increased mortality among female heart attack patients. Proceedings of the National Academy of Sciences of the United States of America 2018 doi: 10.1073/pnas.1800097115 [published Online First: 2018/08/08]

10. Pagidipati NJ, Peterson ED. Acute coronary syndromes in women and men. Nat Rev Cardiol 2016;13(8):471-8o. doi: 10.1038/nrcardio.2016.89

11. Jneid H, Fonarow GC, Cannon CP, et al. Sex differences in medical care and early death after acute myocardial infarction. Circulation 2008;118(25):2803-10. doi: 10.1161/ CIRCULATIONAHA.108.78980o [published Online First: 2008/12/10]

12. Izadnegahdar $M$, Norris $C$, Kaul $P$, et al. Basis for sex-dependent outcomes in acute coronary syndrome. Can J Cardiol 2014;30(7):713-20. doi: 10.1016/j.cjca.2013.08.020 [published Online First: 2014/01/29] 
13. Milner KA, Funk M, Richards S, et al. Gender differences in symptom presentation associated with coronary heart disease. Am J Cardiol 1999;84(4):396-9.

14. Pilote L, Karp I. GENESIS-PRAXY (GENdEr and Sex determInantS of cardiovascular disease: From bench to beyond-Premature Acute Coronary SYndrome). Am Heart J 2012;163(5):74146 e2. doi: 10.1016/j.ahj.2012.01.022

15. Canto JG, Goldberg RJ, Hand MM, et al. Symptom presentation of women with acute coronary syndromes: myth vs reality. Arch Intern Med 2007;167(22):2405-13. doi: 10.1001/ archinte.167.22.2405

16. Dey S, Flather MD, Devlin G, et al. Sex-related differences in the presentation, treatment and outcomes among patients with acute coronary syndromes: the Global Registry of Acute Coronary Events. Heart 2009;95(1):20-6. doi: 10.1136/hrt.2007.138537 [published Online First: 2008/05/09]

17. Rubini Gimenez M, Twerenbold R, Boeddinghaus J, et al. Clinical Effect of Sex-Specific Cutoff Values of High-Sensitivity Cardiac Troponin T in Suspected Myocardial Infarction. JAMA Cardiol 2016;1(8):912-20. doi: 10.1001/jamacardio.2016.2882 [published Online First: 2016/og/23]

18. Shah AS, Griffiths M, Lee KK, et al. High sensitivity cardiac troponin and the underdiagnosis of myocardial infarction in women: prospective cohort study. BMJ 2015;350:g7873. doi: 10.1136/bmj.g7873

19. Trambas C, Pickering JW, Than M, et al. Impact of High-Sensitivity Troponin I Testing with Sex-Specific Cutoffs on the Diagnosis of Acute Myocardial Infarction. Clinical chemistry 2016;62(6):831-8. doi: 10.1373/clinchem.2015.252569 [published Online First: 2016/04/28]

20. Wu AHB, Christenson R. The Era for High-Sensitivity Cardiac Troponin Has Begun in the US (Finally). The Journal of Applied Laboratory Medicine: An AACC Publication 2017;2(1):13. doi: 10.1373/jalm.2017.023648

21. Thygesen K, Alpert JS, Jaffe AS, et al. Fourth universal definition of myocardial infarction (2018). European heart journal 2018 doi: 10.1093/eurheartj/ehy462 [published Online First: 2018/08/31]

22. Eggers KM, Apple FS, Lind L, et al. The applied statistical approach highly influences the $99^{\text {th }}$ percentile of cardiac troponin I. Clinical biochemistry 2016;49(15):1109-12. doi: 10.1016/j. clinbiochem.2016.08.012

23. Twerenbold R, Boeddinghaus J, Nestelberger T, et al. Clinical Use of High-Sensitivity Cardiac Troponin in Patients With Suspected Myocardial Infarction. Journal of the American College of Cardiology 2017;70(8):996-1012. doi: 10.1016/j.jacc.2017.07.718

24. Roffi M, Patrono C, Collet JP, et al. 2015 ESC Guidelines for the management of acute coronary syndromes in patients presenting without persistent ST-segment elevation: Task Force for the Management of Acute Coronary Syndromes in Patients Presenting without Persistent ST-Segment Elevation of the European Society of Cardiology (ESC). European heart journal 2016;37(3):267-315. doi: 10.1093/eurheartj/ehv320

25. Thygesen K, Alpert JS, Jaffe AS, et al. Third universal definition of myocardial infarction. Journal of the American College of Cardiology 2012;6o(16):1581-98. doi: 10.1016/j. jacc.2012.08.001

26. Reichlin T, Hochholzer W, Bassetti $S$, et al. Early diagnosis of myocardial infarction with sensitive cardiac troponin assays. N Engl J Med 2009;361(9):858-67. doi: 10.1056| NEJMoaogoo428 [published Online First: 2009/08/28] 
27. Keller T, Zeller T, Peetz D, et al. Sensitive troponin I assay in early diagnosis of acute myocardial infarction. N Engl J Med 2009;361(9):868-77. doi: 10.1056/NEJMoaog03515 [published Online First: 2009/08/28]

28. Mueller C. Biomarkers and acute coronary syndromes: an update. European heart journal 2014;35(9):552-6. doi: 10.1093/eurheartj/eht530

29. Shah ASV, Anand A, Strachan FE, et al. High-sensitivity troponin in the evaluation of patients with suspected acute coronary syndrome: a stepped-wedge, cluster-randomised controlled trial. Lancet 2018 doi: 10.1016/So140-6736(18)31923-8 [published Online First: 2018/o9/02]

30. Hickman PE, Koerbin G, Potter JM, et al. Statistical considerations for determining highsensitivity cardiac troponin reference intervals. Clinical biochemistry 2017;50(9):502-05. doi: 10.1016/j.clinbiochem.2017.02.022

31. Sandoval Y, Apple FS. The global need to define normality: the $99^{\text {th }}$ percentile value of cardiac troponin. Clinical chemistry 2014;6o(3):455-62. doi: 10.1373/clinchem.2013.211706

32. van Doorn WPTM, Vroemen WPH, De Boer D, et al. Clinical laboratory practice recommendations for high-sensitivity cardiac troponin testing. J Lab Precis Med 2018 doi: 10.21037/jlpm.2018.03.09

33. Lippi G, Aloe R, Meschi $\mathrm{T}$, et al. Interference from heterophilic antibodies in troponin testing. Case report and systematic review of the literature. Clinica chimica acta; international journal of clinical chemistry 2013;426:79-84. doi: 10.1016/j.cca.2013.09.004 [published Online First: 2013/09/18]

34. Bais R. The effect of sample hemolysis on cardiac troponin I and $\mathrm{T}$ assays. Clinical chemistry 2010;56(8):1357-9. doi: 10.1373/clinchem.2010.144139 [published Online First: 2010/06/o8]

35. Dimeski G, Coogan M, Jones B, et al. Is the new Beckman AccuTnI+3 assay capable of producing false-positive troponin I results? Clinical chemistry and laboratory medicine 2015;53(4):e101-3. doi: 10.1515/cclm-2014-0616 [published Online First: 2014/09/27]

36. Ryan JB, Southby SJ, Stuart LA, et al. Comparison of cardiac TnI outliers using a contemporary and a high-sensitivity assay on the Abbott Architect platform. Ann Clin Biochem 2014;51(Pt 4):507-11. doi: 10.1177/0004563214534637 [published Online First: 2014/04/24]

37. Warner JV, Marshall GA. High incidence of macrotroponin I with a high-sensitivity troponin I assay. Clinical chemistry and laboratory medicine 2016;54(11):1821-29. doi: 10.1515/cclm2015-1276 [published Online First: 2016/04/20]

38. Wildi K, Gimenez MR, Twerenbold R, et al. Misdiagnosis of Myocardial Infarction Related to Limitations of the Current Regulatory Approach to Define Clinical Decision Values for Cardiac Troponin. Circulation 2015;131(23):2032-40. doi: 10.1161/ CIRCULATIONAHA.114.014129

39. Mueller T, Egger M, Peer E, et al. 5 th generation cardiac troponin I and $\mathrm{T}$ assays in clinical routine - A head-to-head comparison with data from the Linz troponin (LITROP) study. Clinica chimica acta; international journal of clinical chemistry 2018;485:195-204. doi: 10.1016/j.cca.2018.06.027 [published Online First: 2018/07/01]

40. Mueller T, Egger M, Peer E, et al. Evaluation of sex-specific cut-off values of high-sensitivity cardiac troponin I and T assays in an emergency department setting - Results from the Linz Troponin (LITROP) study. Clinica chimica acta; international journal of clinical chemistry 2018 doi: 10.1016/j.cca.2018.09.026 [published Online First: 2018/09/19] 
41. Hammarsten O, Mair J, Mockel M, et al. Possible mechanisms behind cardiac troponin elevations. Biomarkers 2018:1-26. doi: 10.1080/1354750X.2018.1490969 [published Online First: 2018/07/07]

42. Haaf $\mathrm{P}$, Reichlin $\mathrm{T}$, Twerenbold $\mathrm{R}$, et al. Risk stratification in patients with acute chest pain using three high-sensitivity cardiac troponin assays. European heart journal 2014;35(6):36575. doi: 10.1093/eurheartj/eht218 [published Online First: 2013/07/04]

43. Omland T, de Lemos JA, Holmen OL, et al. Impact of sex on the prognostic value of highsensitivity cardiac troponin I in the general population: the HUNT study. Clinical chemistry 2015;61(4):646-56. doi: 10.1373/clinchem.2014.234369 [published Online First: 2015/02/20]

44. Hijazi Z, Siegbahn A, Andersson U, et al. Comparison of cardiac troponins I and $\mathrm{T}$ measured with high-sensitivity methods for evaluation of prognosis in atrial fibrillation: an ARISTOTLE substudy. Clinical chemistry 2015;61(2):368-78. doi: 10.1373/ clinchem.2014.226936

45. deFilippi C, Seliger SL, Kelley W, et al. Interpreting cardiac troponin results from highsensitivity assays in chronic kidney disease without acute coronary syndrome. Clinical chemistry 2012;58(9):1342-51. doi: 10.1373/clinchem.2012.185322

46. Freda BJ, Tang WH, Van Lente F, et al. Cardiac troponins in renal insufficiency: review and clinical implications. Journal of the American College of Cardiology 2002;40(12):2065-71.

47. Abbas NA, John RI, Webb MC, et al. Cardiac troponins and renal function in nondialysis patients with chronic kidney disease. Clinical chemistry 2005;51(11):2059-66. doi: 10.1373/ clinchem.2005.055665

48. Buiten MS, de Bie MK, Rotmans JI, et al. Serum Cardiac Troponin-I is Superior to Troponin-T as a Marker for Left Ventricular Dysfunction in Clinically Stable Patients with End-Stage Renal Disease. PloS one 2015;10(8):e0134245. doi: 10.1371/journal.pone.0134245 [published Online First: 2015/08/04]

49 van der Linden $\mathrm{N}$, Cornelis $\mathrm{T}$, Kimenai DM, et al. Origin of Cardiac Troponin $\mathrm{T}$ Elevations in Chronic Kidney Disease. Circulation 2017;136(11):1073-75. doi: 10.1161/ CIRCULATIONAHA.117.029986 [published Online First: 2017/09/13] 


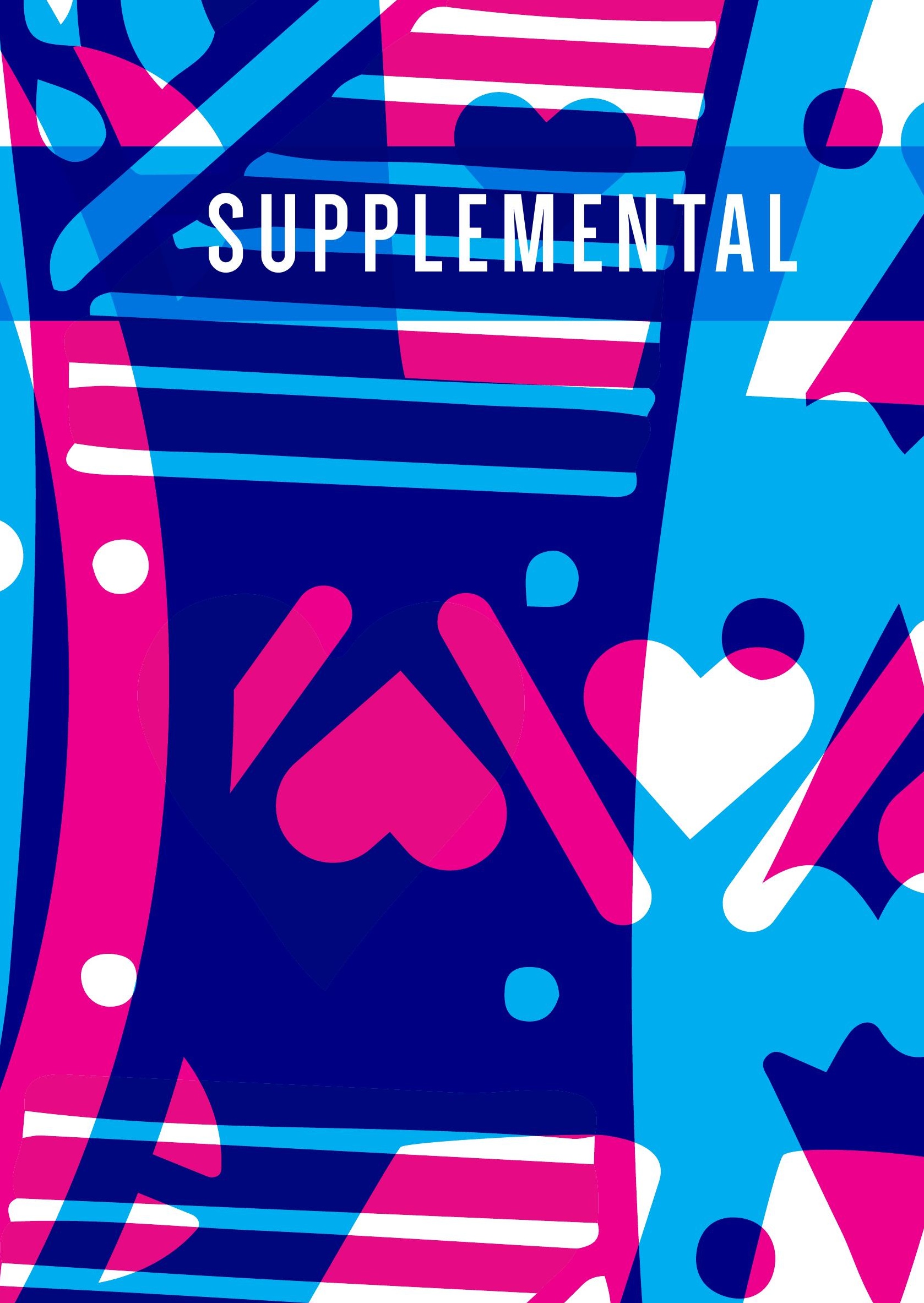




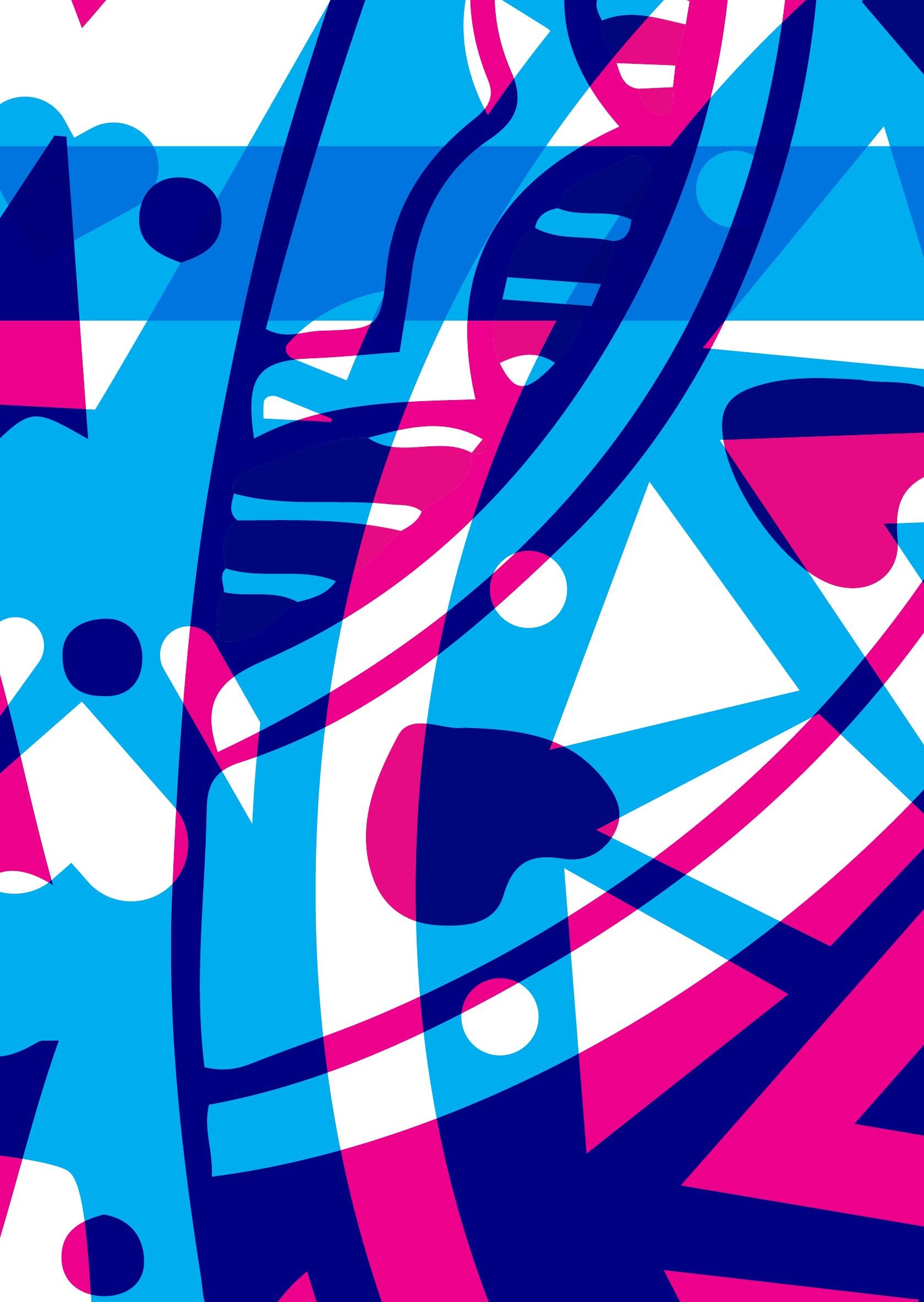


SUPPLEMENTAL 


\section{SUMMARY}

The cardiac troponin I and $\mathrm{T}$ biomarkers are incorporated in the algorithm for diagnosis of acute myocardial infarction (AMI). Cardiac troponin assays have been improved in their sensitivity over the last years. The current guidelines recommend these so-called high-sensitivity cardiac troponin I (hs-cTnI) and $\mathrm{T}$ (hs-cTnT) assays over the contemporary troponin assays for diagnosis of AMI. At this point, the common AMI diagnostic threshold is the $99^{\text {th }}$ percentile upper reference limit (URL) of hs-cTn from a healthy reference population. The increased sensitivity of hs-cTn assays revealed previously unrecognized presence of cardiac troponin concentration differences between women and men. However, whether this resulted into different female-specific and male-specific $99^{\text {th }}$ percentile URLs of hs-cTn was unexplored. Furthermore, the $99^{\text {th }}$ percentile URLs of hs-cTnI and hs-cTnT were derived from different reference cohorts and determined by different statistical approaches. The impact of population heterogeneity and inconsistent statistical approach on $99^{\text {th }}$ percentile URLs of hs-cTnI and hs-cTnT has not been systematically investigated.

In this thesis two main topics were investigated associated with the use of hs-cTn assays:

\section{>FEMALE VERSUS MALE HEARTS}

Sex-related aspects of cardiac biomarkers, in particular the $99^{\text {th }}$ percentile upper reference limits of high-sensitivity cardiac troponins for the diagnosis of an acute myocardial infarction.

\section{> BATTLE OF THE CARDIAC TROPONINS}

The (dis)concordance between high-sensitivity cardiac troponin I and $\mathrm{T}$ in respect of $99^{\text {th }}$ percentile upper reference limits, and their underlying associations with cardiac injury and kidney function in the non-acute setting. 
CHAPTER 2 shows that female-specific $99^{\text {th }}$ percentile URLs of hs-cTn are lower than male-specific $99^{\text {th }}$ percentile URLs of hs-cTn (hs-cTnI: 11 versus $20 \mathrm{ng} / \mathrm{L}$, hscTnT: 12 versus $16 \mathrm{ng} / \mathrm{L}$ ). Direct comparison of hs-cTnI and hs-cTnT revealed that the hs-cTnI distribution is more profoundly affected by extreme outliers than hs-cTnT. The $99^{\text {th }}$ percentile URLs of hs-cTnI and hs-cTnT derived from a single well-phenotyped healthy reference population $(\mathrm{n}=1540)$ are numerically similar (13 versus $15 \mathrm{ng} / \mathrm{L}$ ), suggesting that the currently applied overall clinical decision limit of $26 \mathrm{ng} / \mathrm{L}$ for hs-cTnI may lead to underdiagnosis of AMI in women.

Our systematic review (CHAPTER 3) confirmed that female-specific thresholds of hs-cTn, particularly hs-cTnI, are lower than package insert clinical decision limits of $26 \mathrm{ng} / \mathrm{L}$ and $14 \mathrm{ng} / \mathrm{L}$, respectively. Furthermore, we extended our findings of CHAPTER 2 on hs-cTnI outliers, and show that hs-cTnI-thresholds are more heterogeneous across studies than hs-cTnT-thresholds. Direct comparison of hs-cTnI-hs-cTnT thresholds from single healthy reference populations revealed that handling of outliers, rather than population heterogeneity, seems the critical factor for determination of an appropriate $99^{\text {th }}$ percentile URL for hs-cTnI.

CHAPTER 4 is an overview of the literature of sex-related aspects of cardiac biomarkers which are used in acute cardiac care setting, with a focus on cardiac troponins for diagnosis of AMI and NT-proBNP for diagnosis of heart failure (HF). This literature study shows that clinical trials investigating the relevance of sex-specific thresholds of hs-cTn for diagnosis of AMI is controversial and needs further investigation. Contrary to hs-cTn, clinical research has shown that negative predictive values for ruling-out HF were hardly effected when applying a universal diagnostic threshold that is independent from sex or other risk factors.

In CHAPTER 5, we directly compared hs-cTnI and hs-cTnT in relation to (subclinical) cardiac injury in an ambulant setting. In contrast to the strong hs-cTnI-hs-cTnT correlation in the acute cardiac care setting, we demonstrate a poor correlation $(\mathrm{r}=0.585)$ and limited concordance of hs-cTnI-hs-cTnT ( $\geq$ sexspecific $75^{\text {th }}$ percentile, $\mathbf{K}=0.397$ ) in a non-acute setting. Isolated elevations of troponin I, but not isolated troponin T levels, are associated with (subclinical) cardiac injury measured by electrocardiographic changes indicative of cardiac abnormalities. Altogether, our data suggest that different mechanisms drive troponin I and $\mathrm{T}$ elevations in the chronic setting.

CHAPTER 6 shows that in a population without substantial chronic kidney disease (CKD), estimated Glomerular Filtration Rate (eGFR) and albuminuria are already independently associated with both hs-cTn at levels that do not fulfill the CKD criteria. Though marginal, eGFR is more strongly associated with hscTnT than with hs-cTnI. The underlying mechanisms and the relevance of this difference needs further study. This study supports the concept that eGFR and albuminuria are, over their entire range, associated with cardiac injury. 
CHAPTER 7 discusses our key findings in the perspective of current knowledge. Clinical workup is needed to investigate the relevance of using sex-specific $99^{\text {th }}$ percentile URLs of hs-cTnI and hs-cTnT for diagnosis of AMI, and whether it effectively reduce underdiagnosis of AMI in women. Future hs-cTn strategies for diagnosis of AMI may go beyond $99^{\text {th }}$ percentile URLs, whereby further study on the clinical relevance of applying sex-specific hs-cTn algorithms needs urgent attention. The observed discordance between hs-cTnI and hs-cTnT needs further study in longitudinal studies. Investigating the usefulness of hs-cTn in preventive strategies should clarify whether the hs-cTnI-hs-cTnT differences also translates into relevant differences on prediction of cardiac morbidity and mortality. 


\section{DUTCH SUMMARY (NEDERLANDSE SAMENVATTING)}

De cardiale biomarkers troponine I en $\mathrm{T}$ zijn één van de hoekstenen in het diagnostisch algoritme van een hartinfarct. De cardiaal troponine metingen zijn in de loop van de jaren verbeterd in hun gevoeligheid. De huidige richtlijnen bevelen deze zogenaamde hoog-sensitieve cardiaal troponine I (hs-cTnI) en $\mathrm{T}$ (hs-cTnT) metingen aan boven de oudere generaties cardiaal troponine metingen voor het vaststellen van hartinfarct. De $99^{\text {ste }}$ percentiel waarden van hs-cTnI en hs-cTnT van een gezonde referentie populatie zijn de huidige afkapwaarden voor de diagnose van een hartinfarct. De verhoogde gevoeligheid van hs-cTn metingen onthulden de tot daarvoor niet herkenbare verschillen in cardiaal troponine concentraties tussen vrouwen en mannen. Of deze sekse verschillen ook daadwerkelijk resulteren in verschillende vrouw-specifieke $99^{\text {ste }}$ percentiel waarden en man-specifieke $99^{\text {ste }}$ percentiel waarden van hs-cTnI en hs-cTnT was tot op heden nog niet systematisch bestudeerd. Daarnaast zijn de $99^{\text {ste }}$ percentiel van hs-cTnI en hs-cTnT afkomstig van verschillende gezonde referentie populaties en op basis van een verschillende statistische aanpak berekend. De impact van populatie heterogeniteit en verschillende statische toepassingen op de $99^{\text {ste }}$ percentiel waarden van hs-cTnI en hs-cTnT zijn tot dusver niet systematisch onderzocht.

In dit proefschrift hebben we twee onderdelen bekeken gerelateerd aan het gebruik van hs-cTn metingen:

\section{> VROUWENHART VERSUS MANNENHART}

Sekse-gerelateerde aspecten van cardiale biomarkers, met in het bijzonder de $99^{\text {ste }}$ percentiel waarden van hoog-sensitieve cardiaal troponines voor de diagnose van een hartinfarct.

\section{> DE STRIJD TUSSEN DE CARDIALE TROPONINES}

De (dis)concordantie tussen hoog-sensitieve cardiaal troponine I en T met betrekking tot $99^{\text {ste }}$ percentiel waarden, en hun onderliggende relaties met cardiale schade en nierfunctie in de niet-acute setting. 
HoOFDSTUK 2 toont dat de vrouw-specifieke $99^{\text {ste }}$ percentiel waarden van hs-cTn lager zijn dan de man-specifieke $99^{\text {ste }}$ percentiel waarden van hs-cTn (hs-cTnI: 11 versus 20 $\mathrm{ng} / \mathrm{L}$, hs-cTnT: 12 versus $16 \mathrm{ng} / \mathrm{L})$. Een directe vergelijking tussen hs-cTnI en hs-cTnT laat zien dat de hs-cTnI distributie gevoeliger is voor extreme uitbijters dan hs-cTnT. De $99^{\text {ste }}$ percentiel waarden van hs-cTnI en hs-cTnT die berekend en afkomstig zijn vanuit één gezonde referentie populatie $(\mathrm{n}=1540)$ zijn numeriek vergelijkbaar (13 versus $15 \mathrm{ng} / \mathrm{L}$ ), wat suggereert dat de huidig gebruikte diagnostische afkapwaarde van $26 \mathrm{ng} / \mathrm{L}$ van hs-cTnI mogelijk leidt tot onderdiagnose van een hartinfarct bij vrouwen.

In ons systematische literatuuronderzoek (HoOFDSTUK 3) bevestigen we dat de vrouw-specifieke afkapwaarden van hs-cTn, met name hs-cTnI, lager zijn dan de klinisch gebruikte algemene diagnostisch afkapwaarden van $26 \mathrm{ng} / \mathrm{L}$ voor hs-cTnI en $14 \mathrm{ng} / \mathrm{L}$ voor hs-cTnT. Daarnaast hebben we de bevinding van meer hs-cTnI uitbijters dan hs-cTnT uitbijters nader bestudeerd, en zien we een grotere heterogeniteit tussen studies naar hs-cTnI afkapwaarden dan bij studies die hs-cTnT afkapwaarden bestudeerd hebben. Uit onderzoek naar hs-cTnI-hs-cTnT afkapwaarden die afkomstig zijn van één gezonde referentie populatie lijkt met name de wijze waarop met uitbijters van hs-cTnI wordt omgegaan, dé kritische factor te zijn om een correcte $99^{\text {ste }}$ percentiel van hs-cTnI vast te stellen.

HoofDSTUK 4 is een overzicht van de literatuur over sekse verschillen van cardiale biomarkers die worden gebruikt in de acute cardiale setting, specifiek gericht op de cardiaal troponines voor de diagnose van een hartinfarct en NT-proBNP voor de diagnose van hartfalen. Deze literatuurstudie toont dat studies die hebben gekeken naar de klinische relevantie van het gebruik van sekse-specifieke afkapwaarden van hs-cTn voor de diagnose van een hartinfarct controversiële resultaten tonen. Toekomstig onderzoek om hier uitsluitsel over te krijgen is noodzakelijk. In tegenstelling tot hs-cTn, hebben klinische studies voor NT-proBNP aangetoond dat de negatief voorspellende waarden bij het gebruik van een algemene diagnostische afkapwaarde nauwelijks beïnvloed werden door sekse of andere risicofactoren.

In HOOFDSTUK 5 hebben we hs-cTnI en hs-cTnT direct met elkaar vergeleken in relatie tot (subklinische) cardiale schade in een niet-acute setting. In tegenstelling tot de eerder gevonden sterke correlatie tussen hs-cTnI en hs-cTnT in de acute setting, demonstreren wij een matige correlatie $(r=0.585)$ en beperkte concordantie van hs-cTnI-hs-cTnT ( $\geq$ sekse-specifiek $75^{\text {ste }}$ percentiel, $\mathrm{K}=0.397$ ) in de niet-acute setting. Geïsoleerde hs-cTnI verhogingen, maar niet geïsoleerde hs-cTnT verhogingen, zijn geassocieerd met (subklinische) cardiale schade welke is gemeten door ECG-veranderingen die op cardiale abnormaliteiten duiden. Onze observaties suggereren dat er verschillende mechanismes ten grondslag liggen aan troponine I en $\mathrm{T}$ verhogingen in de niet-acute setting.

HoofDSTUK 6 toont in een populatie zonder substantiële chronische nierziekten (CKD) dat de geschatte Glomerulaire Filtratie Snelheid (eGFR) en albuminurie ook al 
onafhankelijk geassocieerd zijn met cardiaal troponine I en T, zelfs bij een geschatte nierfunctie waarbij nog geen sprake is van CKD. Hoewel het verschil marginaal is, is eGFR sterker geassocieerd met hs-cTnT dan hs-cTnI. Toekomstig onderzoek naar de mechanisme(n) die hieraan ten grondslag liggen en de relevantie van dit verschil is nodig. Onze studie ondersteunt de hypothese dat eGFR en albuminurie over de gehele range zijn geassocieerd met cardiale schade.

In HOOFDSTUK 7 bediscussiëren we onze hoofdbevindingen in het licht van de huidige bestaande kennis. Toekomstig onderzoek naar de klinische relevantie van het toepassen van sekse-specifieke $99^{\text {ste }}$ percentiel waarden van hs-cTnI and hs-cTnT is actueel en relevant. Toekomstige hs-cTn strategieën voor de diagnostiek van een hartinfarct gaan mogelijk voorbij aan de $99^{\text {ste }}$ percentiel waarden van hs-cTn. Ook bij alternatieve algoritmes is onderzoek naar de klinische relevantie van het toepassen van sekse-specifieke hs-cTn algoritmen van belang. De geobserveerde discordantie tussen hs-cTnI en hs-cTnT behoeft verder onderzoek in longitudinale studies. Het bestuderen van de toegevoegde waarde van hs-cTn in preventie strategieën moeten duidelijkheid geven of de gevonden hs-cTnI-hs-cTnT verschillen ook resulteren in relevante verschillen op het voorspellen van cardiale morbiditeit en mortaliteit. 


\section{VALORIZATION}

\section{$>$ RELEVANCE}

Around 2012 most Dutch hospitals implemented high-sensitivity cardiac troponin (hs-cTn) assays in their laboratory to establish diagnosis of acute myocardial infarction (AMI). The common AMI diagnostic threshold is the $99^{\text {th }}$ percentile upper reference limit (URL) of hs-cTn from a healthy reference population. During the implementation phase of hs-cTn assays in Europe, no attention was paid to sex-specific hs-cTn thresholds. Also European manufactures of hs-cTn assays inconsistently report sex-specific cutoffs. As a result, most Dutch hospitals apply overall hs-cTn $99^{\text {th }}$ percentile URLs for AMI diagnosis. This thesis revealed that $99^{\text {th }}$ percentiles URLs of hs-cTnI and hs-cTnT differ markedly between sexes, reflecting in lower female-specific $99^{\text {th }}$ percentile URLs than men-specific $99^{\text {th }}$ percentile URLs, which may contribute to underdiagnosis of AMI in women.

\section{$>$ TARGET GROUPS}

This thesis is of interest for all crucial stakeholders that are involved in the clinical decision making of AMI diagnosis; physicians working at emergency department, internists, cardiologists and clinical laboratory specialists. In addition, our research might be also of interest for health insurance companies, diagnostic industry, social impact consultants and patients, as translating the results into a social cost benefit analysis helps to understand the potential broader social impact of sex-specific hscTn thresholds such as potential societal cost savings. Apart from AMI diagnosis, also the general practitioners may read this thesis with interest, as hs-cTn is a promising biomarker for preventive strategies.

\section{$>$ PRODUCT}

Our research revealed that women have lower $99^{\text {th }}$ percentile URLs of hs-cTn than men. In addition, we show that the statistical approach rather than reference population heterogeneity contributes to current discordant clinical decision limits of hs-cTnI and hs-cTnT.

\section{$>$ IMPLEMENTATION}

Future research is needed to investigate whether a sex-specific algorithm of hs-cTn leads to a better identification and treatment of AMI in women, and subsequently, is required to improve prognosis in women after AMI. In addition, further study to downward adjustment of overall clinical decision limit of hs-cTnI for AMI diagnosis in relation to outcome needs urgent attention.

\section{> WOMEN ARE NOT MEN AND MEN ARE NOT WOMEN, FORTUNATELY}

During my PhD, I studied the (unmet) area of sex- and gender inequalities. I was surprised that basically each person, including myself, is biased on this matter. E.g. sex discrimination in regard to career perspectives is in most cases not based 
on bad intentions, but rather on the lack of self-knowledge of your own sex-bias. I'm deeply grateful to the people in the past who have dared to stand up for women's rights at a time that it was not yet self-evident. Their huge statements have led to awareness, have been balanced and weighted over time, and will definitely lead to equal opportunities for women and men in the future. Now, four years later, with much more knowledge about sex- and gender inequalities, I hope that the results in this thesis will contribute to better healthcare for women and men. 


\section{LIST OF ABBREVIATIONS}

$\begin{array}{ll}\text { aa } & \text { Amino acids } \\ \text { ACS } & \text { Acute coronary syndrome } \\ \text { ADP } & \text { Accelerated diagnostic protocol } \\ \text { AMI } & \text { Acute myocardial infarction } \\ \text { ANP } & \text { Atrial natriuretic peptide } \\ \text { AP } & \text { Angina pectoris } \\ \text { ARIC } & \text { the atherosclerosis risk in communities study } \\ \text { BMI } & \text { Body mass index } \\ \text { BNP } & \text { Brain-type natriuretic peptide } \\ \text { BP } & \text { Blood pressure } \\ \text { CABG } & \text { Coronary artery bypass graft } \\ \text { CAC } & \text { Coronary artery calcification } \\ \text { CAD } & \text { Coronary artery disease } \\ \text { CAMELIA } & \text { Cardiovascular risks metabolic syndrome Liver and autoimmune } \\ \text { CHF } & \text { Congestive heart failure } \\ \text { CHS } & \text { Canberra heart study } \\ \text { CI } & \text { Confidence interval } \\ \text { CK } & \text { Creatine kinase } \\ \text { CKD } & \text { Chronic kidney disease } \\ \end{array}$




\begin{tabular}{|c|c|}
\hline CKD-EPI & Chronic kidney disease epidemiology collaboration \\
\hline CKMB & Creatine kinase muscle-brain type \\
\hline CNP & C-type natriuretic peptide \\
\hline cTn & Cardiac troponin \\
\hline cInl & Cardiac troponin I \\
\hline cInT & Cardiac troponin T \\
\hline CV & Coefficient of variation \\
\hline CVD & Cardiovascular disease \\
\hline DHS & the Dallas heart Study \\
\hline DM & Diabetes mellitus \\
\hline DNP & D-type natriuretic peptide \\
\hline ECG & Electrocardiography \\
\hline EF & Ejection fraction \\
\hline eGFR & Estimated glomerular filtration rate \\
\hline ESC & European society of cardiology \\
\hline FDA & Food and drug administration \\
\hline FN & False negative \\
\hline FP & False positive \\
\hline GHS & Gutenberg health study \\
\hline GUIDE-IT & Guiding evidence based therapy using biomarker intensified treatment in heart failure \\
\hline HDL & High density lipoprotein \\
\hline HF & Heart failure \\
\hline HFpEF & Heart failure with preserved ejection fraction \\
\hline HFrEF & Heart failure with reduced ejection fraction \\
\hline hs-cTn & High-sensitivity cardiac troponin \\
\hline hs-cTnl & High-sensitivity cardiac troponin I \\
\hline
\end{tabular}




\begin{tabular}{|c|c|}
\hline hs-cTnT & High-sensitivity cardiac troponin T \\
\hline IFG & Impaired fasting glucose \\
\hline IGT & Impaired glucose tolerance \\
\hline IQR & Interquartile range \\
\hline LDL & Low density lipoprotein \\
\hline LN & Natural log-transformed \\
\hline LOB & Limit of blank \\
\hline LOD & Limit of detection \\
\hline LOQ & Limit of quantitation \\
\hline LV & Left ventricular \\
\hline LVH & Left ventricular hypertrophy \\
\hline LVMI & Left ventricular mass index \\
\hline MAREA & Metabolic alterations in Reggio Calabria Adolescents \\
\hline MC & Minnesota coding \\
\hline MHELP & Montignoso heart lung project \\
\hline MONICA & Monitoring of trends and determinants for cardiovascular disease \\
\hline MR-proANP & Mid-regional pro atrial natriuretic peptide \\
\hline NGM & Normal glucose metabolism \\
\hline NGT & Normal glucose tolerance \\
\hline NOBIDA & Nordic reference interval project bio-bank and database \\
\hline NP & Nonparametric \\
\hline NPV & Negative predictive value \\
\hline NT-proBNP & N-terminal pro brain-type natriuretic peptide \\
\hline NSEW & North south east west \\
\hline NSTEMI & Non-ST-elevation myocardial infarction \\
\hline OR & Odds ratio \\
\hline
\end{tabular}




$\begin{array}{ll}\text { PCI } & \text { Percutaneous coronary intervention } \\ \text { PIVUS } & \text { Prospective investigation of the vasculature in Uppsala seniors } \\ \text { PPV } & \text { Positive predictive value } \\ \text { PRISMA } & \text { Preferred reporting items for systematic reviews and meta-Analyses } \\ \text { OI } & \text { 25't quartile } \\ \text { O3 } & \text { 75' }{ }^{\text {th }} \text { quartile } \\ \text { ROC } & \text { Receiver operating characteristic } \\ \text { RV } & \text { Right ventricular } \\ \text { SD } & \text { Standard deviation } \\ \text { SHBG } & \text { Sex hormone-binding globulin } \\ \text { STEMI } & \text { ST-elevation myocardial infarction } \\ \text { TIDM } & \text { Type I diabetes mellitus } \\ \text { T2DM } & \text { Type } 2 \text { diabetes mellitus } \\ \text { InC } & \text { Troponin C } \\ \text { InI } & \text { Troponin I } \\ \text { InT } & \text { Troponin T } \\ \text { TN } & \text { True negative } \\ \text { TP } & \text { True positive } \\ \text { TSH } & \text { Thyroid stimulating hormone } \\ \text { URL } & \text { Upper reference limit } \\ \text { UA } & \text { Unstable angina } \\ \text { UAE } & \text { Urinary albumin excretion } \\ \text { VEGF } & \text { Vascular endothelial growth factor } \\ \text { NO } & \text { Nitric oxide } \\ \end{array}$




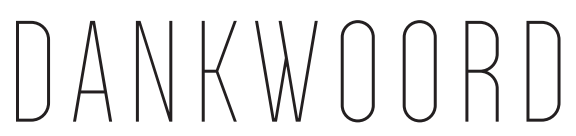

Dit dankwoord schrijvende doet me realiseren dat ik heb durven kiezen voor een ( $h$ )eerlijk leven, dat uiteindelijk nog mooier is dan ik ooit heb durven dromen. Ik ben dankbaar dat ik tijdens mijn promotietraject met een groep gedreven mensen heb mogen samenwerken die me met een kritische maar ook altijd respectvolle houding behandeld hebben.

\section{$>$ PROMOTIE TEAM.}

Dr. Meex, beste Steven, je hebt als copromotor mijn onzekerheid over mijn gemixte achtergrond omgezet in een positief gevoel waardoor ik daar ongelooflijk trots op ben geworden. Jouw bevlogenheid in onderzoek maakte dat ik iedere week weer uitkeek naar onze research meeting om samen aan een vraagstuk te puzzelen. Je hebt me de ruwe kanten van het vak nooit onthouden, waardoor ik me bewust ben geworden van de haat-liefde verhouding die $i k$ aanga wanneer ik kies voor een carrière in de academie. Door jou ben ik ook gaan zien dat je als wetenschapper bevoorrecht bent om nieuwsgierigheid alle ruimte te geven. Het vrijheidsgevoel wat daaraan gekoppeld is, was precies datgene waar ik naar op zoek was. Dankjewel Steven. Gelukkig hoef ik je bulderende lach voorlopig nog niet te missen! Professor Bekers, beste Otto, door jouw visie heb ik mogen werken op een afdeling die staat voor een betrokken, professionele en collegiale afdeling. Ik heb bewondering voor de wijze waarop jij de saamhorigheid op het CDL waarborgt. Ik heb geleerd van je diplomatieke werkwijze en kan het bijzonder waarderen dat je me op dat gebied een aantal keer op weg geholpen hebt. De twinkeling die jij dan altijd in je ogen kreeg wanneer ik om je hulp vroeg, was overigens onbetaalbaar. Veel dank dat ik mijn weg voorlopig nog even op het CDL mag vervolgen.

Professor van Dieijen-Visser, beste Marja, dat je uiteindelijk niet tot mijn promotie team behoorde was een begrijpelijke keuze. Veel dank voor de stevige en inspirerende (troponine) fundering die je mij, alsook de afdeling, gegeven hebt.

\section{$>$ PARANIMFEN.}

Lieve Judith, roomie en paranimf, je hebt me wegwijs gemaakt in het Mooie Maastricht en door jou ben ik me er thuis gaan voelen. Samen zijn we gestart op het CDL in onze "bezemkast" en wat kijk ik met plezier terug op de periode dat we samen een huisje gedeeld hebben. Dankzij jouw onvoorwaardelijke vrolijke houding zijn er vele geluksmomenten geweest, was je mijn steun en toeverlaat, en ik had dit alles dan ook niet zonder jou willen 
en kunnen doen. Ik geloof dat onze visie "een goed resultaat moet worden gevierd met foute drank" inmiddels gedeeld wordt met de huidige collega promovendi $\odot$. Lieve Judith, wat fijn dat ik je heb leren kennen. Met jouw geluksgen, oprechtheid, enthousiasme en liefheid ga jij een verdomd goede internist worden, succes met de opleiding!

Lieve Eefje, hardloopmaatje en paranimf. Ik geloof niet dat wij op voorhand voorzien hadden dat onze hardloopprojecten zulke wijze levenslessen met zich meedroegen. Ik heb door onze avonturen leren lef (en lief) te hebben, maar ook om goed voor mezelf te zorgen en daarmee grenzen te ontdekken die ik met mijn leven ben gaan bewaken. Vrijheid vond ik ook bij jou. Samen met de kever en enkel een wegenkaart door Frankrijk rijden was fantastisch! Wat hebben we gelachen, op 1 moment na $:$, en wat was de Champagne héérlijk! Dankjewel lieve Pattyn voor de fijne gesprekken, heerlijke stiltes en ontzettend goede grappen tijdens de vele kilometers die we samen hebben afgelegd. Ik blijf je mijn hele wetenschappelijke carrière nodig hebben en hoop dat je nog heel lang mijn partner in crime blijft op jacht naar avontuurrr.

Tomas, dank je wel voor al die lekkere koffie die altijd klaarstond en luisterend oor. Met jouw creativiteit is het gelukt om mijn proefschrift te presenteren in een inspirerende vorm. Ik kan wel stellen dat ik door jou en Eefje, van de details buiten mijn promotie om, overeind ben blijven staan. Fijn dat ik in mijn proefschrift jullie blauwdruk terugzie.

\section{> STAFLEDEN EN KCIO'S VAN HET CDL.}

Douwe de Boer, Alma Mingels, Yvonne Henskens, Judith Bons, Jan Damoiseaux, Irene Körver-Keularts, Bart de Wit, Will Wodzig, Joyce van Beers en Sander Streng.

Ook de KCio's die hun pad inmiddels hebben vervolgd; Henrike Hamer, Marianne Zijlstra en Kelly Broen. Ik heb me door jullie op mijn plek gevoeld de afgelopen jaren. Bedankt voor alle refereersessies, wetenschapsmiddagen en de fijne samenwerkingen die er zijn geweest! Douwe, ik hoop dat ik de verborgen schat met al je doping verhalen ooit nog eens weet te vinden! Alma, een bijzonder woord van dank aan jou. Jij bent de eerste geweest die vrouwman verschillen van cardiaal troponine $T$ rapporteerde en ik vond het dan ook een eer toen je me vroeg of $i k$ samen met jou het boekhoofdstuk wilde schrijven. Ik vond het erg leuk samenwerken! Maastrichts Mooiste samen lopen vond ik ook overigens ook erg leuk \#driemusketiers. Sander, fijn dat je je weg op het CDL hebt kunnen vervolgen als KCio. Dank dat ik je altijd als hulplijn kon inzetten wanneer ik weer eens computerproblemen had. Ook aan alle andere collega's van het CDL, mercikes!

\section{$>$ BEOORDELINGSCOMMISSIE.}

Prof. dr. Maurice Zeegers, prof. dr. Christa Cobbaert, dr. Vanessa van Empel, prof. dr. Manuela Joore en prof. dr. Ron Kusters, hartelijk dank dat jullie de tijd en moeite hebben willen nemen om mijn manuscript te beoordelen.

\section{$>$ MANAGEMENT TEAM VAN DE MAASTRICHT STUDIE.}

Prof.dr. Coen Stehouwer, dr. Carla van der Kallen, dr. Annemarie Koster, dr. Miranda Schram, prof. dr. Pieter Dagnelie, prof. dr. Nicolaas Schaper, en met in het bijzonder dr. Ronald Henry, 
bedankt voor de prettige samenwerking. Door de projecten bij De Maastricht Studie heb ik mijn epidemiologische skills verder kunnen ontwikkelen. Ik heb jullie wetenschappelijk integere werkwijze bijzonder gewaardeerd, een voorbeeld.

\section{$>$ DE PROJECTGROEP GENDER \& GEZONDHEID.}

Dr. Yolande Appelman, dr. Hester den Ruijter, dr. Christine Swart, Xanne Visser, Jeroen Crasborn, Vincent Belgraver en Marlon van Dijk. Aanjullie allen: ongelooflijk veel dank!Jullie hebben mij uit de troponine bubbel getrokken, me bewust gemaakt van het maatschappelijke belang, alsook laten zien dat samenwerken goud waard is. Yolande, ik realiseer me maar al te goed dat ik met het onderzoek naar vrouw-man verschillen op een rijdende trein ben gestapt die door jouw volhardende inzet de afgelopen jaren in beweging is gebracht. Hester, ik vond je een inspirerende wetenschapper en mijn eerste sollicitatie brief voor een promotieplek ging dan ook naar jou (niet eens uitgenodigd om op gesprek te komen (-)). Ik heb tóch de kans gekregen met je te mogen gaan samenwerken. Ik vind jullie beiden (nog steeds) inspirerende wetenschappers! Het is ons nu uiteindelijk gelukt om het verzekeraarsproject van de grond te krijgen. Een mooier vervolg had ik me niet kunnen wensen.

\section{$>$ COLLEGA ONDERZOEKERS.}

Vincent, wat heb jij door de jaren heen al heel wat promovendi zien komen en gaan. Jouw aanwezigheid zorgt ervoor dat de onderzoekers de weg op het lab weten te vinden. Ik vond het dan ook erg fijn dat je altijd bereid was mee te denken over de praktische vragen. Ook heel erg bedankt voor de plezierige samenwerking op het lab, waarbij we samen ontelbaar veel metingen hebben verricht, het was iedere keer weer gezellig!

Emma, ik heb een kleine glimlach moeten onderdrukken toen ik hoorde dat je mijn project verkoos boven dat van het afdelingshoofd. Wat was je een fantastische student! Ik heb een iets grotere glimlach moeten onderdrukken toen je zei dat je ging starten aan de master Epidemiologie en graag op mijn aanbod inging om weer terug te komen op het CDL. Ik vind het ontzettend leuk dat we op dit moment weer samen aan een vrouw-man-troponine vraagstuk aan het puzzelen zijn! Wat mooi dat we binnenkort een getalenteerd epidemioloog rijker zijn.

Frederique, klinische vraagstukken vind ik geweldig, dus bedankt dat je me liet aansluiten bij jouw studies. Ik vond je een hele fijne collega waar ik altijd op heb kunnen terugvallen. Je oog voor detail en directe, lieve en respectvolle houding maakt jou een geweldige dokter waar heel veel dokters een voorbeeld aan kunnen nemen. Succes met de opleiding tot cardioloog!

Linda, ook bij jouw studies mocht $i k$ regelmatig mee puzzelen. Jij hebt me het duwtje in de juiste richting gegeven. Dankjewel voor de fijne gesprekken over werk en alles wat daar omheen zit $:-$. Leuk om te zien dat je je plekje nu in het noorden van het land helemaal gevonden hebt. Succes met de opleiding tot neuroloog!

Wim, William en Stephanie, met veel liefde hebben jullie altijd voor de volle potten koffie en de gezelligheid op het kantoor gezorgd! Heel veel dank! Wim en William, het duo dat 
ook altijd paraat stond bij sportieve uitjes en mij weer lab werk liet uitvoeren (onder jullie supervisie weliswaar (-))! Fantastisch mannen! Wim, toen jij het design "randomized controlled trail" bij mij introduceerde, werd ik direct getriggerd. Ik vind het ook een goed idee dat we (lees: Steven, Wim, William en ik) volgend jaar de Hivernaltrail gaan lopen waarbij we random ingedeeld gaan worden voor de afstanden $11 \mathrm{~km}, 19 \mathrm{~km}, 30 \mathrm{~km}$ of $50+\mathrm{km}$. William, het was heel erg leuk om je te zien ontwikkelen van de jonge student tot de (bijna) volwassen vent die je nu bent. Blijf vooral enorm van het leven genieten! En de Wilhelmina berg trappen marathon op 5 oktober 2019 hebben jullie ook al in je agenda genoteerd toch? Stephanie, succes met het afronden van je proefschrift. Eline, Lieke en Noreen, bedankt dat jullie me als ervaren AIO's wegwijs hebben gemaakt in de troponine wereld. Petal, jij bent me er ook eentje $(-)$, dankjewel voor je gezellige aanwezigheid op het lab. Anne-Hilde en Minka, $i k$ vond het leuk jullie te leren kennen. Succes met alles wat er op jullie pad komt!

Mike en Glenn, ik had me geen fijnere collega-onderzoekers kunnen wensen! Mike, beiden hadden we geen zekerheid over ons vervolgtraject, maar door die ervaring te delen werd het op geen moment een zorgelijk vooruitzicht, no worries $-\dot{-}$. Glenn, jij hebt je verworven promotieplek in Luik dik verdiend! Succes met alles.

Joris, wat was je voor mij een inspirerend persoon. Ik heb in een korte tijd bijzonder veel van je opgestoken. Ik hoop dat onze wegen nog eens kruisen.

\section{$>$ COAUTEURS.}

Dr. Simone Sep, dr. Marijke Niens, dr. Jeroen van Suijlen, dr. Remy Martens, prof. $d r$. Jeroen Kooman, dr. Frans Tan, prof. dr. Bram Kroon, prof. dr. Karel Leunissen en dr. Frank van der Sande. Dank voor jullie wetenschappelijke bijdrage aan mijn studies, het heeft geresulteerd in afgewogen artikelen. I also gratefully thank prof. dr. Bertil Lindahl and $d r$. Kai Eggers for the pleasant and fruitful collaboration. I look forward to our next research project and working together again. Dear prof. dr. Paul Kerkhof and prof. dr. Virginia Miller, thank you very much for the given opportunity to contribute to your beautiful book on sexbased cardiovascular research.

\section{$>$ VRIENDEN.}

De afgelopen vier jaar zijn loslaten en ontdekken hand in hand gegaan, het is net wetenschap. Onmisbaar.

Mijn kever maatjes Marjolein en Bart, jullie zijn me even dierbaar als mijn kever, iets wat ik nooit zal loslaten. Ik kan genieten van de wijze waarop jullie de eenvoud in het leven omarmen en hard werken om jullie dromen waar te maken. Op naar nog heel veel mooie momenten samen!

Ronnie, een vriendschap die ik voor geen goud zou willen missen, het verliezen met squashen overigens wel $\odot$. Fabiënne, ik hoop dat jijje fanatisme en mooie emoties voor altijd behoudt. Fijn dat ik altijd nog zo'n heerlijke lunch voorgeschoteld krijg nadat ik afgemat ben door Ron. Marla en Tom, met een grote glimlach ben ik afgelopen jaar met mijn kever \& daktent de 
camping opgereden en ben ik een hele gezellige avond met jullie tegemoet gegaan. Sanne, bij jou durfde ik voor het eerst weer kwetsbaar te zijn na een lange tijd. Hoe jij je gezin, werk en opleiding combineert vind ik bewonderenswaardig. En Thomas, dankzij jouw barbecue kunsten ben ik met een grote glimlach de laatste dag van mijn vakantie naar huis gereden! Geniet van jullie mooie gezinnetjes! Dankjulliewel voor alles.

Mijn HAVO vrienden Monique, Karlijn en Ton, ik kijk er altijd weer naar uit om jullie te zien! Leuk dat onze verschillende levensverhalen nog een paar keer per jaar resulteren in beregezellige avonden.

Het Lumière clubje, de Papendal cursus was zo slecht nog niet :-). Het heeft geresulteerd in gezellige film avonden waar ik altijd weer vrolijk van thuis kwam. Genevieve, succes met de opleiding tot radioloog! Charlotte, jouw lieve open houding gaat je op een heel mooi pad brengen.

Kampeerclub 't Juultje; Strid, Eefje, Miran, Soof, Lisa en Chris. Ben ik nu iemand vergeten? Door jullie voel ik me altijd weer heel normaal. Ik ben dankzij jullie nooit de thuisbasis kwijtgeraakt. Strid, je bent een vriendin die iedereen wel zou willen hebben, wat ben ik blij met onze (h)eerlijke vriendschap! Over eerlijkheid gesproken, Miran, jij moet me beloven qua persoonlijkheid nooit te veranderen. Soof, jou op Bonaire opzoeken samen met Jip was bijzonder fijn, ik kijk terug op een waanzinnig mooie duik samen! Lisa, wat heb jij veel kilometers afgelegd om bij mijn 30 ste verjaardags verrassingsfeestje in Maastricht te kunnen zijn, mijn mooiste verjaardag ooit $(-)$. En Chris, kerstavond was de afgelopen jaren door jouw goede zorgen tijdens het schuurmetten onvergetelijk! Ik ben dankbaar dat jullie de afgelopen jaren als een front voor mij hebben klaargestaan.

Eline en Tessel, de Vereniging voor Epidemiologie heeft ons gekoppeld en verbonden, en wat ben ik blij dat ikjullie heb leren kennen! Eline, bijjou mag ik gewoon zijn, wat een groot goed. Tessel, ik keek altijd weer uit naar onze telefonische overleggen om de activiteiten voor de junior epidemiologen te organiseren, en we gaan momenten creëren waarbij we onze "overleggen" kunnen voortzetten!

Vincent Callewaert, officieel bestaat mijn promotieteam uit twee leden, maar eigenlijk heb ik drie begeleiders gehad. Als sportfysiotherapeut was jij de backbone van mijn hardloopprojecten en heb jij (onbewust) een cruciale rol gespeeld in mijn promotie. Mijn intrinsieke motivatie is door de combinatie sport en wetenschap vele malen versterkt. Op sportgebied heb je mij laten zien hoe ik ondanks tegenslagen kan genieten en gaan voor mijn doelen. Het mooie is dat die regels 1 op 1 over te nemen zijn in de wetenschap. Thanks Vincent, zonder jou was ik nooit zo intens genietend bij die ultraloop én bij mijn proefschrift over de eindstreep gekomen.

\section{$>$ FAMILIE}

Karin, Manon, Miranda, Frank, Johan en Peter. Een gezin met vele verschillen, maar met de ondernemendheid als overeenkomst. Door de bedrijvigheden van de familie Kimenai - hout hakken, vrachtwagens, MTB of herten verplaatsen over de weg in de Klein-Dongen buurt- 
zal ik, gelukkig, nooit een "statige" academicus worden. Het is fijn dat ik door jullie soms even uit de complexe onderzoekswereld kan stappen, terug die zalige wereld van eenvoud in.

Lieve Kunie en Henk, bedankt voor jullie bijdrage aan mijn onbezorgde jeugd. Ik kijk met veel plezier terug op de vakanties samen met jullie in Schoorl! Ik ben blij dat jullie onderdeel zijn van ons gezin.

Lieve Mam en Pap, tijdens mijn promotietijd ben ik pas gaan inzien dat jullie altijd en onvoorwaardelijk voor mij paraat staan. Jullie zijn in dit alles de fundering geweest, ik hou van jullie. Altijd hebben jullie me op het hart gedrukt dat je risico's mag nemen en vooral moet durven kiezen voor datgene wat je leuk vind. Nou Mam en Pap: dit vond ik leuk! Heel veel liefs.

Als laatste wil ik toch eigenlijk echt mezelf bedanken. Ik ben mezelf dankbaar voor alle fouten, onhandigheden en verkeerde inschattingen die ik de afgelopen jaren heb gemaakt (het waren er nogal wat $:)$ ). Het heeft geresulteerd dat dit proefschrift een prachtig ongeschreven hoofdstuk bevat die mij inzicht heeft gegeven in de waardevolle elementen van het leven: liefde, respect, integriteit, eerlijkheid en vrijheid. Het heeft me leren vallen, maar vooral leren opstaan. Het heeft bijgedragen dat ik als wetenschapper nu weet te verliezen en mij gevormd tot een dankbare intens gelukkige vrouw. 


\section{ABOUT THE AUTHOR}

Dorien Kimenai was born on March 15, 1987 in Klein-Dongen, The Netherlands. She graduated in 2004 from Secondary school(HAVO) at Cambreur College in Dongen. From 2004 to 2008 she studied Biology and Medical Laboratory Research at Avans University of Applied Sciences in Breda. When she obtained her bachelor's degree, she started in 2009 with the in-service training to Clinical Perfusionist of which she defended her thesis in February 2012 at LUMC in Leiden. Dorien worked from 2012 to 2014 as clinical perfusionist. Meanwhile, she followed the master program Clinical Epidemiology at the AMC-UvA in Amsterdam and finalized her master thesis cum laude. In September 2014, Dorien started her PhD-program at the department of Clinical Chemistry/ Central Diagnostic Laboratory at Maastricht UMC+ within the CARIM school for Cardiovascular Diseases, under the supervision of dr. Steven Meex and prof. dr. Otto Bekers. During this period, she conducted various teaching activities such as teacher SPSS skills for the bachelor's Biomedical Sciences and Health Sciences and she was coordinator and teacher of the Clinical Chemistry Course for the master Biomedical Engineering. Since 2016, Dorien is secretary and board member of the Netherlands Epidemiology Society, and has got the opportunity to initiate the working group Junior Epidemiologists. As member of the working group Junior Epidemiologists she aims to inspire and connect junior epidemiologists colleagues in the Netherlands by organizing early career activities. Dorien will continue her work as postdoctoral researcher with pleasure at Maastricht $\mathrm{UMC}+$ for the upcoming year. 
